\title{
XDazl function in RNA metabolism in Xenopus laevis
}

\author{
Doctoral Thesis
}

\author{
Dissertation for the award of the degree \\ "Doctor rerum naturalium (Dr.rer.nat)" \\ in the GGNB program: "Genes and Development" \\ at the Georg August University Göttingen \\ Faculty of Biology
}

submitted by

Juliane Pfennig

born in Erfurt, Germany

Göttingen, September 2014 


\section{Members of the Thesis Committee:}

Supervisor

Prof. Dr. Tomas Pieler (Reviewer),

Dept. of Developmental Biochemistry, Georg August University

Second member of the thesis committee

Prof. Dr. Michael Kessel (Reviewer),

Dept. of Developmental Biology, Max Planck Institute for Biophysical Chemistry

Third member of the thesis committee

Prof. Dr. Matthias Dobbelstein,

Institute of Molecular Oncology, Georg August University

Date of oral examination: 26.11.2014 
Affidavit

Herewith, I declare that I prepared the PhD thesis

"XDazl function in RNA metabolism in Xenopus laevis"

on my own and with no other sources and aids than quoted.

Submission date

Göttingen, 30.09.2014

Juliane Pfennig 


\section{TABLE OF CONTENTS}

\section{INTRODUCTION.}

1.1 Germ cell specification.

1.2 Mechanisms of germ cell development in C. elegans, Drosophila, Danio rerio and Xenopus - germ cell specification and maternal factors

1.2.1 Germ cell development in Caenorhabditis elegans .................................2

1.2.2 Germ cell development in Drosophila melanogaster ...............................2

1.2.3 Germ cell development in Danio rerio ................................................ 4

1.2.4 Germ cell development in Xenopus laevis ............................................. 5

1.2.4.1 Vegetal localization of mRNAs in Xenopus oocytes .............................. 5

1.2.4.2 Formation and development of germ cells in Xenopus embryos .............. 7

1.3 Maternal to zygotic transition and miR-mediated decay in Xenopus and

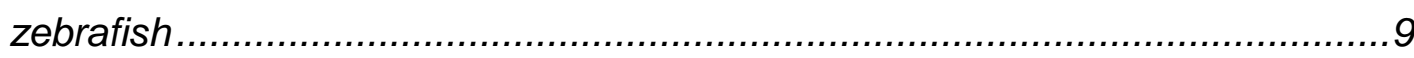

1.4 Germ cell specific RNA protection from miR-mediated decay.......................11

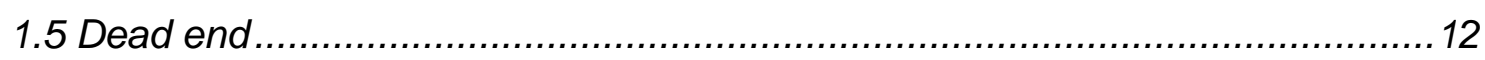

1.6 Hu proteins (ElrA and ElrB1) ........................................................... 13

1.7 Deleted in Azoospermia-like (Dazl)....................................................... 14

1.7.1 Origin and structure of the DAZ family genes ..................................... 14

1.7.2 Developmental expression of Dazl .................................................. 16

1.7.3 Requirement of Dazl in germ cell development .................................17 
1.7.4 Dazl as a translational regulator ................................................... 17

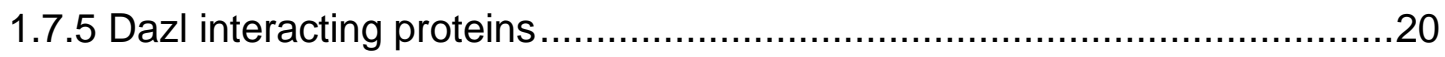

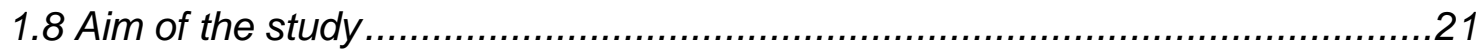

2 MATERIALS AND METHODS

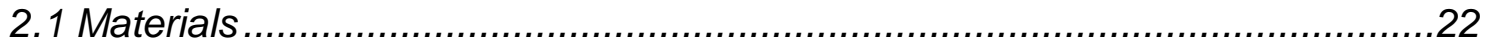

2.1.1 Model Organism Xenopus laevis......................................................22

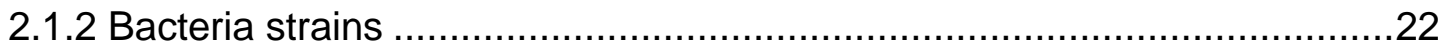

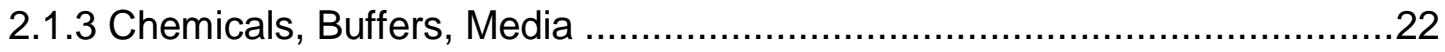

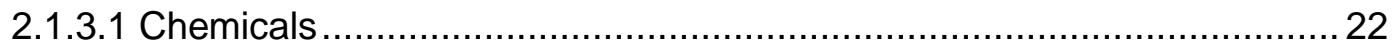

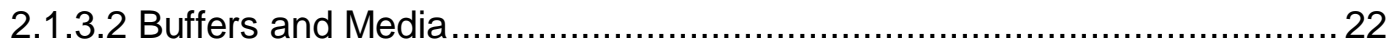

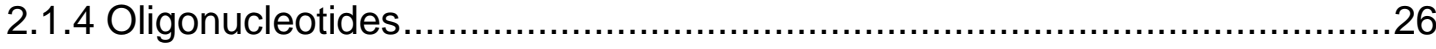

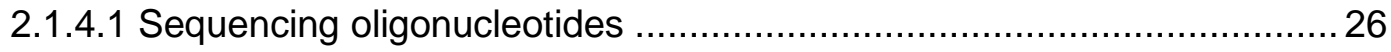

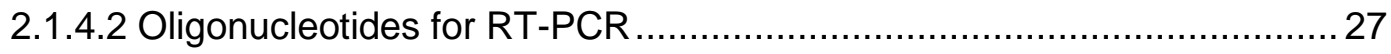

2.1.4.3 Oligonucleotides for the amplification of XDE-LE subfragments .............2 27

2.1.4.4 Oligonucleotides for the amplification of XDazl-LE subfragments........... 28

2.1.4.5 Oligonucleotides for the amplification of XDazI-LE reporter subfragments

2.1.4.6 Oligonucleotides for the $X$. laevis/X. tropicalis hybrid analysis 29

2.1.4.7 Oligonucleotides used for the generation of XDazl ORF deletion mutants

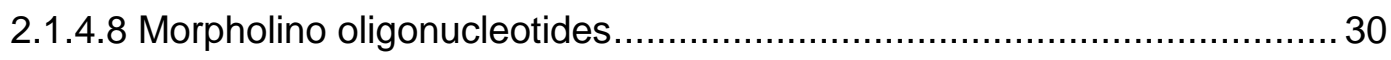

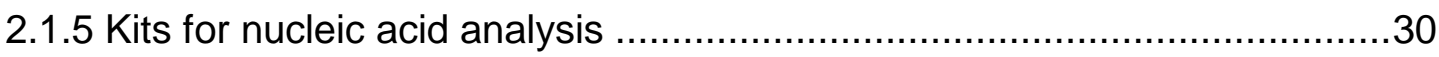

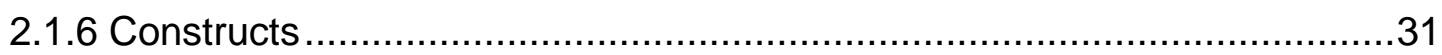

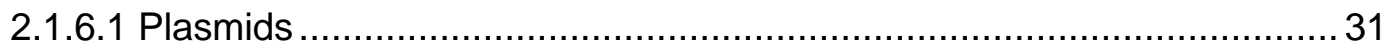

2.1.6.2 Constructs for recombinant protein expression .................................. 32

2.1.6.3 Constructs used for antisense probe transcription ............................. 32

2.1.6.4 Overexpression constructs and reporters........................................... 33

2.1.6.5 Constructs used for the generation of Cy3-labeled RNA ....................... 34

2.1.7 PCR fragments used for the generation of Cy3-labeled RNA..................34

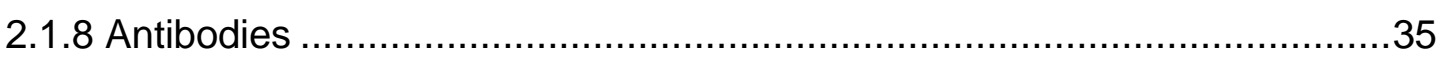

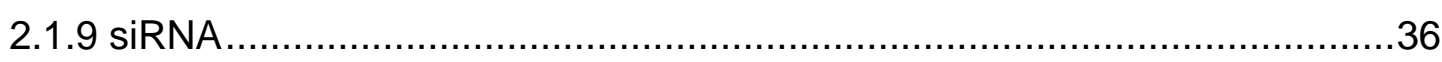

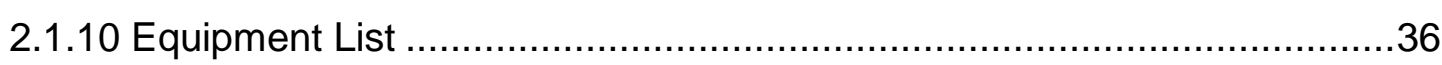

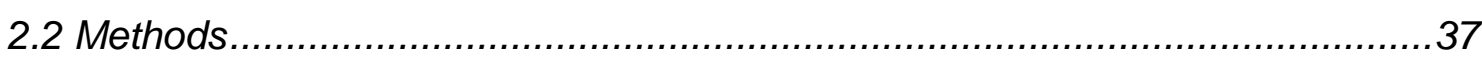

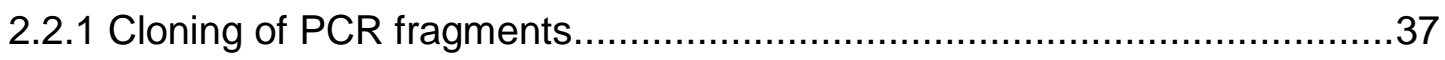




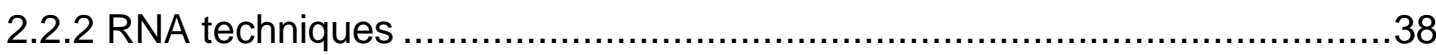

2.2.2.1 In vitro synthesis of capped sense mRNA ......................................... 38

2.2.2.2 In vitro synthesis of Cy3-labeled and antisense RNA ........................... 38

2.2.2.3 Isolation of total RNA with trizol (TriFast) .......................................... 40

2.2.2.4 Reverse transcription (cDNA synthesis) ........................................ 40

2.2.2.5 Semiquantitaive real-time PCR ................................................... 41

2.2.2.6 Quantitative real-time PCR ...................................................... 41

2.2.2.7 Quantitative NanoString nCounter multiplex analysis ........................... 42

2.2.2.8 Whole Mount in situ hybridization (WMISH) ...................................... 42

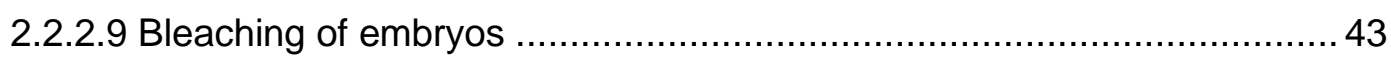

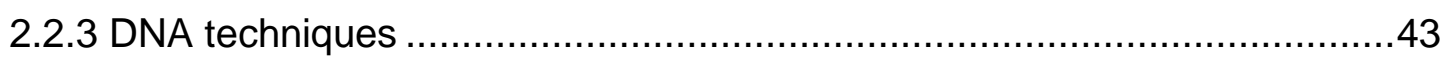

2.2.3.1 Plasmid DNA preparation.............................................................. 43

2.2.3.2 DNA restriction digestion......................................................... 43

2.2.3.3 Agarose gel electrophoresis......................................................... 44

2.2.3.4 DNA fragment isolation from agarose gels or restriction digest ............. 44

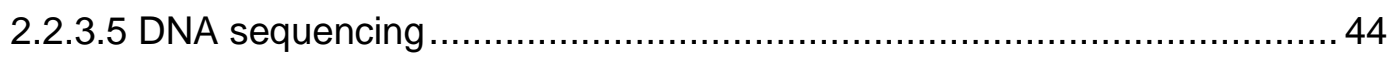

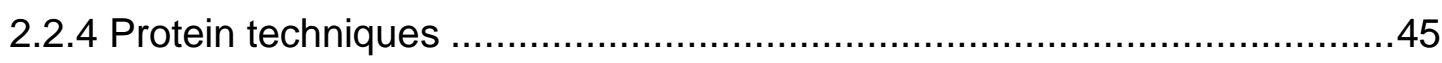

2.2.4.1 Protein isolation from embryos and oocytes ..................................... 45

2.2.4.2 Co-immunoprecipitation using embryo lysates ................................ 45

2.2.4.3 TNT (in vitro transcription coupled translation) .................................... 45

2.2.4.4 Co-immunoprecipitation with in vitro translated proteins and Cy3-labeled RNAs 46

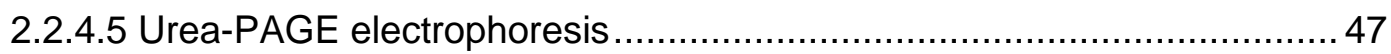

2.2.4.6 Electrophoretic Mobility Shift Assay (EMSA) ..................................... 47

2.2.4.7 SDS-PAGE protein electrophoresis ................................................. 48

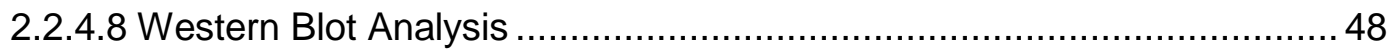

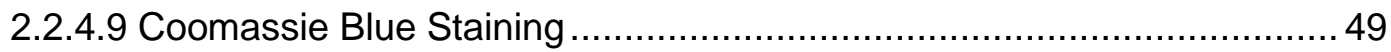

2.2.4.10 Recombinant protein expression and purification .............................. 49

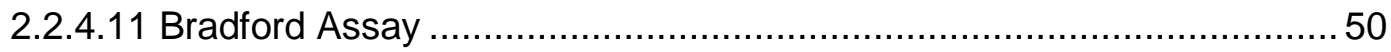

2.2.4.12 Immunofluorescence staining of oocytes ...................................... 50

2.2.5 Xenopus embryo culture and microinjections ......................................51

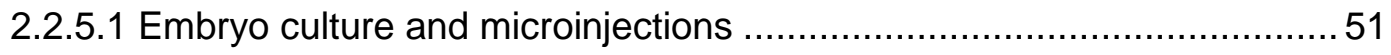

2.2.5.2 Preparation of Xenopus laevis testis ............................................... 52

2.2.5.3 Oocyte culture and microinjection .................................................. 52 
3.1 XDazl mediates protection of germ cell specific mRNAs in Xenopus embryos 53

3.1.1 XDazl stabilizes germ cell specific reporter and endogenous mRNAs in vivo

3.1.2 XDazl functions together with XDE and ElrB1 proteins in somatic XDE-LE reporter RNA stabilization .60

3.2 Binding of XDazl, XDE and ElrB1 proteins to the LEs of germ cell specific mRNAs. 64

3.2.1 XDazl interacts directly with the XDE-LE in vitro and requires an AU-rich region surrounding the mir-18 binding site

3.2.2 Comparison of binding efficiencies of the recombinant proteins XDazl, XDE and ElrB1 to the XDE-LE .66

3.2.3 XDazl has three independent binding sites within the XDazl-LE . .68

3.2.4 XDazl binds directly to the LE of multiple germ cell specific RNAs .70

3.3 Analysis of miRNA responsibility for somatic degradation of XDazl and XDE mRNA 72

3.3.1 Germ cell specific mRNAs are degraded by miR-mediated decay 72

3.3.2 Morpholino protection assay targeting the XDazI-LE s2 fragment revealed two potential miR-target sites .73

3.4 Blocking of zygotic XDazl translation does not affect RNA stability .79

3.5 Colocalization of XDazl protein with germline specific mRNAs at the vegetal cortex in Xenopus oocytes.

3.5.1 XDazl colocalizes with XDE-LE and XDazI-LE at the vegetal cortex of Xenopus oocytes.

3.5.2 The XDazl-LE contains at least three fragments which are sufficient for localization

4 DISCUSSION .92

4.1 XDazl as protector of germ cell specific mRNAs in the embryo 92

4.2 Mechanism of XDazl mediated RNA stabilization .94

4.3 Degradation of maternally provided germ cell specific mRNAs in the zygote is mediated by miRs. .97

4.4 XDazl function in vegetal transport in the oocyte. .99

4.5 Connection of the XDazl function in the oocyte and the embryo. 100 


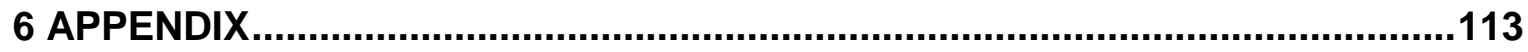

6.1 XDazl binds directly to the XDE-LE in ColP experiments ...........................113

6.2 XDazl binds directly to the XDazI-LE in ColP experiments ...........................114

6.3 Expression of recombinant XDazl protein ................................................. 115

6.4 Expression of recombinant XDE protein .................................................. 115

6.5 Recombinant XDE protein binds to the 5' region in the XDE-LE..................116

6.6 Recombinant ElrB1 binding to the XDE-LE............................................116

$6.7 K_{D}$ values for $X D a z l, X D E$ and ElrB1 protein binding to the analyzed RNA

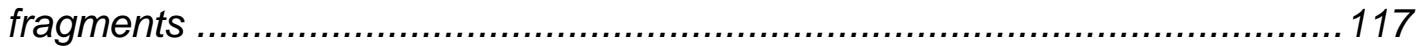

6.8 Summary of RNAs analyzed in NanoString nCounter experiments ..............118

6.8.1 Primary data from NanoString nCounter experiments - ectopic XDazl ...120

6.8.1.1 Processed data from NanoString nCounter analysis - ectopic XDazl ... 121

6.8.2 Primary data from NanoString nCounter experiments - siRNA injection...123

6.8.2.1 Processed data from NanoString nCounter analysis - siRNA injection. 124

6.9 XDE-LE mutant used for the analysis of XDazl, XDE and ElrB1 binding .......126 


\section{ACKNOLEDGEMENTS}

First of all, I want to thank my supervisor Prof. Dr. Tomas Pieler for giving me the opportunity to work on this interesting project and for the great guidance, enthusiastic encouragement and support over these years.

I also want to thank Dr. Maike Claußen for her support and advice on all matters, planning and design of experiments for this research work.

I am very grateful to the members of my thesis committee, Prof. Dr. Michael Kessel and Prof. Dr. Matthias Dobbelstein for critical evaluation and valuable suggestions for this project during committee meetings.

Additionally, I want to thank Prof. Hoyer-Fender, Prof. Wimmer and PD Dr. Shcherbata for being part of my extended thesis committee.

I'm very thankful to the entire Developmental Biochemistry Group for creating a great work environment and all the help and support. I especially want to thank Marion Dornwell, Diana Bauermeister, Aliaksandr Dzementsei, Anita Smarandache, Susanne Koch, Juliane Melchert, Marie Hedderich, Patrick Berndt, Maja Gere, Katja Ditter, Ilona Wunderlich and Franziska Winkler for the great atmosphere, team spirit and encouragement.

I would like to acknowledge the animal care takers, especially Eva Hesse as they made the work with the frogs as pleasant as possible.

My special thanks go to my family, especially my parents, sister and grandparents who always supported and encouraged me throughout my life.

Finally, I would like to particularly thank André Pfennig for his patience, his support and motivation during the long time of studies and PhD. 


\section{ABSTRACT}

Germ cell specification in Xenopus laevis depends on the inheritance of maternal determinants from the oocyte in form of mRNAs and proteins. At the onset of zygotic transcription, maternal mRNAs are subjected to miR-mediated decay. Interestingly, germ cell specific mRNAs are excluded from miR-induced degradation. Recent studies have provided insights into how RNA-binding proteins can modulate miR-mediated regulation of RNA stability. In Xenopus embryos for example, ElrB1 and Dead end (XDE) proteins have been demonstrated to protect germ cell specific mRNAs from miR-mediated clearance. Recognition of these mRNAs by miRs relies on target sites within the localization element (LE), a specific region in the 3' UTR, which is also required for vegetal mRNA transport in the oocyte (Koebernick et al. 2010).

In this study, we addressed the role of Deleted in azoospermia-like (XDazl) in the context of germline specific transcript stabilization in Xenopus embryos. The RNA binding protein XDazl is provided maternally and its zygotic transcription starts at stage 17 . Ectopic expression of XDazl promotes stabilization of the PGC-specific XDE mRNA as well as that of other germline transcripts. In vitro RNA binding assays indicate direct interaction of XDazl with these transcripts. Moreover, a synergistic effect of XDE and XDazl protein in stabilizing germ cell specific mRNAs was observed. Mapping of the XDazl binding site within the XDE-LE indicated overlapping binding sites with XDE and ElrB1. Degradation of germ cell specific mRNAs is mediated by miRs, since blocking of miR processing leads to enrichment of germline transcripts. XDazl protein binds directly to the LE in its own mRNA, which contains at least three independent XDazl binding sites. The knockdown of XDazl in embryos leads to a reduced number of germ cells at tailbud stage, indicating an important role of XDazl during germ cell formation or maintenance. Immunofluorescence staining of oocytes shows XDazl protein enrichment at the vegetal cortex and in transport particles colocalizing with $X D E$ and $X D a z / m R N A s$, suggesting an early involvement of XDazl in the context of vegetal mRNA transport and/or anchoring. 


\section{LIST OF FIGURES}

Figure 1.1. Primordial germ cell development in different model organisms. .................5

Figure 1.2. Model of vegetal mRNA localization in Xenopus oocytes............................7

Figure 1.3. $P G C$ development in $X$. laevis embryos..........................................

Figure 1.4. MiR-mediated mRNA decay of maternal transcripts at maternal-to-zygotic transition (MZT).

Figure 1.5. Mechanism of microRNA (miR) processing.

Figure 1.6. Modulation of miR-mediated repression in germ cells by the RNA-binding proteins DND1 and Dazl. 12

Figure 1.7. Schematic depiction of the 389 aa long ElrB1 protein containing three RRMs. 14

Figure 1.8. Structure and binding partner of DAZ family proteins. 15

Figure 1.9. Model of Dazl mediated translational regulation of target mRNAs during oocyte maturation. 19

Figure 3.1. Ectopic XDazl protein expression protects $X D E-L E$ reporter RNA from somatic clearance in a dose dependent manner.

Figure 3.2. Ectopic expression of XDazl in Xenopus embryos has a stabilizing effect on multiple germ cell specific RNAs. .56

Figure 3.3. XDazl stabilizes endogenous germ cell specific mRNAs. 57

Figure 3.4. The XDazl RNA recognition motif is necessary for the stabilizing function of the XDazl protein.

Figure 3.5. XDazl and XDE protein act synergistically in somatic XDE-LE reporter RNA stabilization.

Figure 3.6. XDazl and ElrB1 protein act in an additive manner in somatic XDE-LE reporter RNA stabilization.

Figure 3.7. XDazl and ElrB1 coprecipitate with ElrA. .63

Figure 3.8. Comparative analysis of $X D a z l$ binding to subfragments of the $X D E-L E \ldots . .65$ Figure 3.9. Comparison of XDE, XDazl and ElrB1 binding to the XDE-LE subfragments.

Figure 3.10. XDazl protein has three independent binding sites within the XDazl-LE. ...69

Figure 3.11. XDazl protein binds directly to the LEs of multiple germ cell specific RNAs in vitro.

Figure 3.12. Blocking of miR processing in Xenopus embryos leads to stabilization of germ cell specific mRNAs. 
Figure 3.13. Ectopic XDazl protein expression protects the reporter RNA of the XDazl$L E$ s2 subfragment from somatic clearance.

Figure 3.14. Somatic clearance of the $X D a z l-L E$ s2 fragment is strongly inhibited upon injection of target protector MO6 and MO7.

Figure 3.15. Multiple miR binding sites can be predicted in the XDazl-LE s2 subfragment.

Figure 3.16. Blocking the miR-18 binding site within the XDazl-LE s2 fragment interferes with XDazl protein binding. 78

Figure 3.17. XDazl protein and mRNA expression from oogenesis to embryogenesis. .80

Figure 3.18. XDazl transcription starts at embryonic stage 17. .82

Figure 3.19. XDazl morpholino blocks the translation of the XDazl protein efficiently in vitro and in vivo. 84

Figure 3.20. XDazl morpholino injection results in a reduced PGC number in embryos. 86 Figure 3.21. XDazl protein colocalizes in Xenopus oocytes with XDE-LE and XDazI-LE RNA .88

Figure 3.22. The $X D a z I-L E$ contains at least three independent localization signals......90 Figure 4.1. XDazl full length localization element contains different potential miR-binding sites. 100

Figure 4.2. Functional connections between vegetal mRNA transport in oocytes and germ cell specific mRNA protection in the embryo. 101

Figure 6.1. XDazl binds directly to the $X D E-L E$ and requires the 5' region of the LE for binding.

Figure 6.2. XDazl binds directly to the XDazl-LE and has at least two independent binding sites within its LE.

Figure 6.3. Recombinant XDazl RRM protein expression and purification. 115

Figure 6.4. Recombinant XDE protein expression and purification. 115

Figure 6.5. Comparative analysis of XDE binding to subfragments of the $X D E-L E \ldots \ldots 116$

Figure 6.6. Comparative analysis of ElrB1 binding to subfragments of the $X D E-L E . \ldots 116$ Figure 6.7. Mutagenesis of putative XDazl, XDE and ElrA/B binding sites in the XDE-LE. 


\section{LIST OF TABLES}

Table 2.1 Sequencing oligonucleotides and working conditions ................................26

Table 2.2 RT-PCR oligonucleotides and working conditions ....................................27

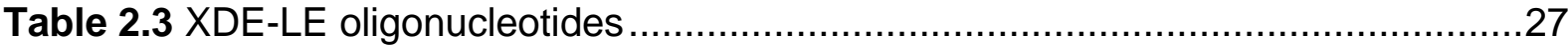

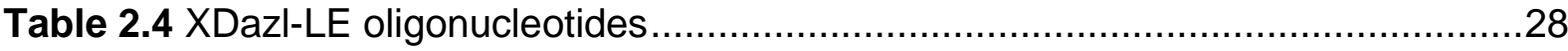

Table 2.5 XDazl-LE oligonucleotides for GFP-reporter cloning ….............................28

Table 2.6 RT-PCR oligonucleotides and working conditions ...................................29

Table 2.7 Oligonucleotides used for the cloning of the XDazl ORF deletion mutants ....29

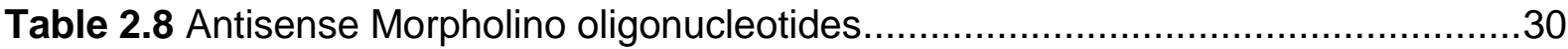

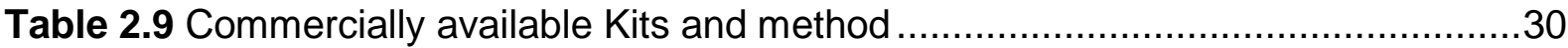

Table 2.10 Expression and reporter constructs - available in the laboratory .................33

Table 2.11 XDazI-LE reporter constructs and in vitro transcription conditions

Table 2.12 Constructs used for the generation of Cy3-labeled RNA and in vitro transcription conditions

Table 2.13 PCR-fragment name and oligonucleotide pair used for the amplification of PCR products applied to the in vitro transcription of XDE-LE Cy3-labeled RNA

Table 2.14 PCR-fragment name and oligonucleotide pair used for the amplification of PCR products applied to the in vitro transcription of XDazl-LE Cy3-labeled RNA. 35

Table 2.15 Primary antibodies. .35

Table 2.16 Secondary antibodies .35

Table 6.1 Summary of all $K_{D}$ values for XDazl, XDE and ElrB1 protein 117

Table 6.2 Summary of RNAs analyzed in NanoString experiments. 118

Table 6.3 Primary data of RNAs analyzed in NanoString experiments - ectopic XDazl

Table 6.4 Fold changes over uninjected embryos of two independent experiments.... 121

Table 6.5 Averaged fold changes over uninjected embryos of two independent experiments and calculated standard deviation

Table 6.6 Primary data of RNAs analyzed in NanoString experiments - siRNA injection

Table 6.7 Fold changes over uninjected embryos of two independent experiments 124

Table 6.8 Averaged fold changes over uninjected embryos of two independent experiments and calculated standard deviation 


\section{ABBREVIATIONS}

A

AP

APS

ARE

Aqua dest.

BA

BB

bp

BCIP

BMB

BSA

C

${ }^{\circ} \mathrm{C}$

cDNA

CHAPS

$\mathrm{cm}$

CoMo

cpm

C-terminus

Cys

d

DAPI

dATP

dCTP

$\mathrm{ddH}_{2} \mathrm{O}$

dGTP

$\mathrm{dH}_{2} \mathrm{O}$

Dig

DMSO

DNA

DNase
Adenine

alkaline phosphatase

Ammonium Persulfate

AU-rich elements

aqua destillata

Benzyl alcohol

Benzyl benzoate

Base pairs

5-bromo-4-chloro-3-indolyl-phosphate

Boehringer Mannheim blocking reagent

Bovine serum albumin

Cytosine

Degrees Celsius

Copy DNA

3- ((3-Cholamidopropyl)dimethylammonio)-1-

Propanesulfonic Acid

Centimeter

Control morpholino

Counts per minute

Carboxy-terminus

Cysteine

day(s)

4',6-Diamidino-2-Phenylindole

Deoxyadenosine triphosphate

Deoxycytosine triphosphate

double distilled water

Deoxyguanosine triphosphate

distilled water

digoxigenine

Dimethylsulphoxid

Deoxyribonucleic acid

Deoxyribonuclease 
dNTP

dT

DTT

dTTP

E. coli

e.g.

EDTA

EGTA

et al.

$\mathrm{EtBr}$

$\mathrm{EtOH}$

$\mathrm{F}$

FCS

Fig.

fw

g

G

Gln

$\mathrm{h}$

hCG

$\mathrm{H}_{2} \mathrm{O}$

$\mathrm{His}$

$\lg$

IPTG

$\mathrm{J}$

K

k

$\mathrm{kb}$

ko

$\mathrm{kd}$

$\mathrm{kD}$

$\mathrm{K}_{\mathrm{D}}$

I

LB

LE
Deoxynucleotide triphosphate

Deoxythymidine

Dithiothreitol

Deoxythymidine triphosphate

Escherichia coli

exempli gratia

Ethylendiamin-tetra-acetic acid

ethylenglycole-bis(2-aminoethylether)-N,N'-

tetraacetate

et alii

Ethidium bromide

Ethanol

forward

Fetal calf serum

Figure

forward

Gram

Guanine

Glycine

Hour

human chorionic gonadotropin

water

Histidine

Immunoglobulin

Isopropyl-thio-galactoside

Joule

Lysine

kilo

Kilo base pairs

knockout

knockdown

Kilodalton

Dissociation constant

Liter

Luria-Bertani

Localization element 
M

$\mathrm{mA}$

MAB

MEM

MEMFA

$\mathrm{mg}$

$\mathrm{MgCl}_{2}$

$\min$

$\mathrm{ml}$

$\mathrm{mm}$

$\mathrm{mM}$

mRNA

$\mathrm{miR}$

MO

$\mu \mathrm{g}$

$\mu l$

$\mu \mathrm{m}$

$\mu \mathrm{M}$

n

$\mathrm{n}$

$\mathrm{NaAC}$

NBT

ng

NLS

$\mathrm{nm}$

$\mathrm{nM}$

NMD

$\mathrm{N}$-terminus

OD

ORF

PAGE

PBS

PCR

PFA

$\mathrm{pH}$
Molar

Milliampere

maleic acid buffer

MOPS-EGTA-MgSO4 buffer

MOPS-EGTA-MgSO4 formaldehyde buffer

Milligram

Magnesium chloride

Minute

Millilitre

Millimeter

Millimolar

messenger RNA

micro RNA

Morpholino

Microgram

Microliter

Micrometer

Micromolar

nano

number

sodium acetate

nitro-blue-tetrazolium

Nanogram

Nuclear localization sequence

Nanometer

Nanomolar

Nonsense mediated decay

Amino-terminus

Optical density

Open reading frame

polyacrylamide gel electrophoresis

Phosphate buffered saline

Polymerase chain reaction

Paraformaldehyde

potentium hydrogenium 
$\%$

Pol

$\mathrm{Pr}$

Pro

PVDF

qRT-PCR

$\mathrm{R}$

$\mathrm{R}$

rev

RNA

RNase

RBP

rpm

RT

RT

RT-PCR

$S$

$S$

SDS

SSC

$\mathrm{sec}$

st

$T$

$T$

TAE

Taq

TBE

TBS(T)

Temp.

Tris

U

UTR

UV

V

$\mathrm{v} / \mathrm{v}$ percentage

Polymerase

Primer

Proline

Polyvinylidene difluoride

Quantitative real-time RT-PCR

Reporter

reverse

reverse

Ribonucleic acid

Ribonuclease

RNA binding protein

Rounds per minute

Room temperature

Reverse transcriptase

Reverse transcription-PCR

Serine

Second

sodium dodecyl sulfate

Standard sodium citrate

second

stage

Thymine

Threonine

Tris-Acetate-EDTA

Thermus aquaticus

Tris-Borate-EDTA

Tris-buffered saline (with Tween)

Temperature

Tris-hydroxymethyl-aminomethane

Units

untranslated region

Ultra violet light

Volume

Volume to volume 
$w / v$

wt

WMISH

$\mathrm{X}$

X-Gal
Weight to volume

wild type

whole mount in situ hybridization

multiple

5-Bromo-4-chloro-3-indoxyl-D-

galactopyranoside 


\section{INTRODUCTION}

Sexually reproducing organisms arise from gametes (sperm and eggs), which are highly specialized cells responsible for the development of a new individual. A small population of cells, namely the germ cells, generates the gametes. Their progenitors, primordial germ cells (PGCs), are set aside from other cell lineages very early in embryonic development in almost all species, including Xenopus (Wylie 1999). As PGCs are essential for the survival of a species, understanding of cellular and molecular mechanisms underlying germ cell formation and maintenance is of particular interest.

\subsection{Germ cell specification}

Although formation of PGCs occurs during early embryonic development the mechanism of germ cell specification is not conserved among animals. Mainly two mechanisms ensure this important developmental event. Germ cells are either specified autonomously by inheritance of cytoplasmic determinants from the egg to a few cells in the dividing embryo (preformation), or their differentiation is induced by inductive signals from neighboring cells (epigenesis). In the majority of species (including salamanders and mammals) germ cells are specified by inductive signals (Extavour and Akam 2003).

In mice, some of these inductive signals have been identified as members of the bone morphogenetic protein (BMP) family (Hogan 1996). Bmp4 and Bmp8b expression in the extraembryonic ectoderm (Lawson et al. 1999, Ying et al. 2000) and Bmp2 transcription in the endoderm (Ying and Zhao 2001) are required for the induction of germ cell fate among proximal epiblast cells and extraembryonic mesoderm. Expression of Wnts by the visceral endoderm enables posterior proximal epiblast cells to respond to BMP signals (Ohinata et al. 2009). In turn, BMPs induce expression of Prdm1 (Blimp1) in a subset of epiblast cells located in the proximal region of the pre-gastrulation mouse embryo, leading to the specification of the germ cell lineage in mice (Ohinata et al. 2005).

The inheritance of maternally provided RNAs and proteins corresponds to the second pathway of PGC determination, preformation. This mechanism is conserved between several model organisms in developmental biology, including C. elegans, Drosophila, Danio rerio and Xenopus. In these species, formation of PGCs requires the presence of a specialized cytoplasmic region called germ plasm. This morphologically distinct structure contains clusters of mitochondria, cisterns of the endoplasmatic reticulum (ER) and electron-dense organelles, and it is called germinal granules in Xenopus or P- 
granules in Drosophila and C. elegans (Kloc et al. 2001). Only those cells that inherit a part of the germ plasm are directed to the germline lineage.

\subsection{Mechanisms of germ cell development in C. elegans, Drosophila, Danio rerio and Xenopus - germ cell specification and maternal factors}

\subsubsection{Germ cell development in Caenorhabditis elegans}

In $C$. elegans, separation of the germline from somatic cells is achieved during the first four embryonic cleavages. The zygote, named $\mathrm{P}_{0}$, divides unequally into a large somatic blastomere $A B$ and a smaller germline blastomere $P_{1}$ containing the $\mathrm{P}$-granules. Whereas the somatic blastomere generates daughter cells by equal division, $P_{1}$ segregates unequally until the $\mathrm{P}_{4} \mathrm{PGC}$ progenitor has been originated. The latter will give rise to two primordial germ cells $Z 2$ and $Z 3$ at about 100-cell stage by symmetrical division. Z2 and Z3 assembles with the gonadal cells Z1 and Z2 to form more than 1000 germ cells in the adult gonad (Figure 1.1 A) (Seydoux and Strome 1999). The germ plasm P-granules comprise several transcriptional inhibitors, e.g. PIE-1 (pharynx and intestine in excess), and RNA-binding proteins such as the orthologs of Drosophila Vasa, Nanos and Piwi (Kawasaki et al. 1998, Seydoux and Strome 1999, Subramaniam and Seydoux 1999). PIE-1 prevents phosphorylation of RNA polymerase II, thereby transiently prohibiting transcription in the germline. In the absence of PIE-1, the germ cell progeny would differentiate into somatic cells, reflecting the importance of transcriptional repression for PGC specification. Until late embryonic stages, PIE-1 protein is active in C. elegans embryos, ensuring germline nuclei silencing (Ghosh and Seydoux 2008). In somatic cells, P-granules are degraded by intervention of MEX-5 and PAR-1 proteins (Brangwynne et al. 2009).

\subsubsection{Germ cell development in Drosophila melanogaster}

In the Drosophila embryo, PGCs are formed as four to five pole cells at the posterior site of the cellularizing blastoderm (Huettner 1930). These cells acquire their identity through inheritance of specialized pole plasm, which assembles around them before fertilization (Mahowald 1971a, b, Schubiger and Wood 1977). Transplantation experiments and forced assembly of pole plasm in ectopic sites led to the formation of PGCs, indicating 
that germ cell determination is mediated by the pole plasm (Mahowald 1971a, b, Illmense.K and Mahowald 1974, Illmensee and Mahowald 1976). One critical factor for germ cell formation is the Oskar protein, which is restricted to the posterior pole. There it localizes mRNAs and proteins required for germ cell formation to the posterior pole. Expression of its mRNA at ectopic sites is sufficient to induce germ plasm assembly and in consequence PGC formation (Ephrussi and Lehmann 1992, Newmark et al. 1997). Early Drosophila embryogenesis starts with syncytial nuclear divisions without cytokinesis. With increasing number of nuclei, transcription is activated in somatic cells, which continue to divide synchronously before they become incorporated into cell membranes (Wieschaus and Sweeton 1988). Further cell divisions are characterized by the ingrowth of a polarized membrane, a process called cellularization (Santos and Lehmann 2004). Similar to $C$. elegans, germ cells in Drosophila are transcriptionally silenced by pole plasm components, inhibiting somatic gene expression during early stages. In contrast to somatic cells, pole cells start asynchronous division and commit germ cell fate after cellularization. The process of PGC formation resembles a budding of the cell membrane (Figure 1.1 B) (Santos and Lehmann 2004). Primarily three genes of the pole plasm, germ-cell less ( $g c l)$, nanos (nos) and polar granule component (pgc) mediate transcriptional/translational regulation. The germ cell-less $(g c l)$ gene is expressed during early cleavage stages. Its encoded protein plays an essential role during pole cell production and prevents gene transcription in the germ line (Leatherman et al. 2002). Nanos protein is important for posterior segment formation and germ cell specification. Pole cells lacking Nanos do not migrate into gonads and fail to become gametes. Furthermore, a role as translational inhibitor of specific mRNAs leading to somatic cell determination was described. In Drosophila Nanos null mutants PGCs undergo apoptosis (Hayashi et al. 2004). Pgc, the third important pole plasm component, mediates transcriptional repression by preventing phosphorylation of the RNApolymerase II (Martinho et al. 2004, Hanyu-Nakamura et al. 2008). Mutations in the pgc gene lead to the expression of transcripts characteristic for somatic cells in PGCs (Martinho et al. 2004). Furthermore, Vasa, Piwi and Aubergine proteins are crucial pole plasm components in the Drosophila embryo (Cox et al. 1998, Megosh et al. 2006). 


\subsubsection{Germ cell development in Danio rerio}

Germ plasm (Balbiani body) formation in zebrafish is mediated by bucky ball mRNA, which also determines polarity (animal-vegetal axis) of oocytes and early embryos (Marlow and Mullins 2008, Bontems et al. 2009). In the dividing embryo, the germ plasm is distributed to four cells by 32-cell stage of embryogenesis and directs them to PGC fate (Yoon et al. 1997, Gao and Arkov 2013). Migration of germ cells to the genital ridge includes relocation to some intermediate target positions to ensure complete cell transfer to sites of gonad formation (Figure 1.1 C) (Kunwar et al. 2006, Dzementsei 2013). Nanos, vasa and piwi mRNAs are germ plasm components in zebrafish, essential for their migration and survival, as well as for the production of oocytes in adult fish. Nanos and Vasa are described as translational regulators, whereas Piwi was shown to silence repetitive elements (Koprunner et al. 2001, Draper et al. 2007, Houwing et al. 2007). Besides these proteins, alterations in chromatin structure, translational changes, mRNA and protein turnover and reconfiguration in the cell-surface proteome are responsible for germline determination (Gustafson and Wessel 2010, Lai et al. 2012). Initiation of migration correlates with transcriptional activation in germ cells (Blaser et al. 2005). The transition from non-motile behavior to directed migration during early PGC development requires the translational regulator Dead end (Dnd). Knockdown of Dnd in the embryo leads to disturbed germ cell polarity and migration, resulting in PGC death through apoptosis (Weidinger et al. 2003). 
A. C.elegans

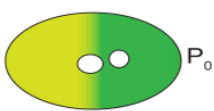

One cell

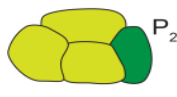

Four cells

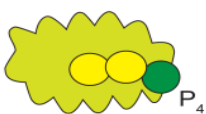

16-24 cells

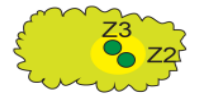

-100 cells

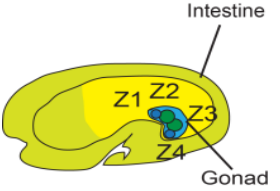

- 550 cells
B. Drosophila

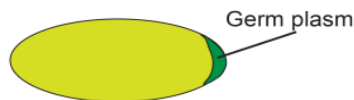

Stage 1-2

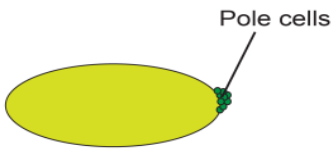

Stage 4-5

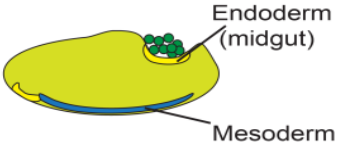

Stage 6-8

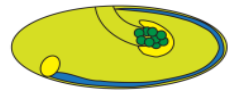

Stage 9-10

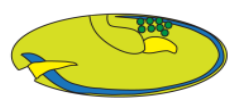

Stage 11-13

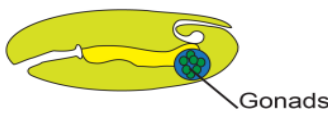

Stage 14
C. Zebrafish

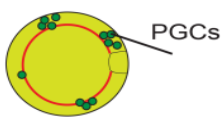

Dome,

animal view

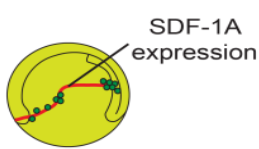

$80 \%$ epiboly

lateral view

left side

anterior

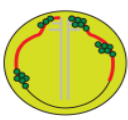

2 somites

frontal view

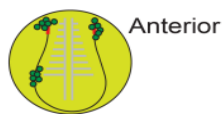

8 somites

frontal view

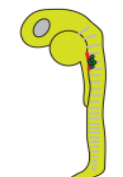

$$
\begin{gathered}
24 \text { hrs } \\
\text { post- } \\
\text { fertilization, } \\
\text { lateral view, } \\
\text { left }
\end{gathered}
$$

Figure 1.1. Primordial germ cell development in different model organisms.

A) In the early $C$. elegans embryo the germ plasm (dark green) is localized towards the posterior site. During the first embryonic cleavages the germ plasm is asymmetrically inherited by the germline blastomeres P1-P4. At 100 cell stage equal division of the germline progenitor blastomere P4 gives rise to two primordial germ cells (PGCs), Z2 and Z3, which form the gonads together with the somatic gonad precursor cells, Z1 and Z4 (yellow).

B) In Drosophila, germ plasm (dark green) assembles at the posterior pole of the oocyte and is inherited to pole cells after fertilization. During germ band extension these cells are transported inside the embryo, specified to PGCs and associate with midgut epithelium cells. PGCs migrate through the midgut towards the mesoderm (blue), and assemble with somatic gonadal cells to generate embryonic gonads.

C) In zebrafish, specified PGCs are transferred to four locations before they migrate to the dorsal side. During gastrulation, they migrate along the expression pattern of the chemoattractant SDF-1a (red), move anteriorly towards somites 1-3, and then to somites 8-10, where they form the gonads together with somatic cells (modified from Dzementsei, 2013).

\subsubsection{Germ cell development in Xenopus laevis}

\subsubsection{Vegetal localization of mRNAs in Xenopus oocytes}

Germ cell specification in Xenopus laevis starts during oogenesis by localization of essential maternal determinants to the vegetal cortex, where they become part of the germ plasm (Houston and King 2000b). In the last decades, many germ plasm associated mRNAs were identified. 
Often they encode for RNA-binding proteins, suggesting their involvement in the regulation of the mRNA metabolism (Kloc et al. 2001). Two major localization pathways have been described in Xenopus oocytes, an early and a late transport mechanism. The first is also called messenger transport organizer (METRO) pathway, which localizes mRNAs encoding germ cell components. In stage I oocytes, mRNAs accumulate in a structure called mitochondrial cloud (MC) at the vegetal site of the nucleus (Kloc and Etkin 1995). At stage I/II of oogenesis, germ cell specific mRNAs like Xcat2 (also known as nanos1), Xpat, XDeadSouth and XDazl migrate together with the $\mathrm{MC}$ to the vegetal pole (Elinson et al. 1993, Mosquera et al. 1993, Hudson and Woodland 1998a, MacArthur et al. 1999, Houston and King 2000a, MacArthur et al. 2000). By stage II/III the $\mathrm{MC}$ breaks into smaller components, which become anchored in a small region at the cortex (Figure 1.3 A + B, left part - oogenesis). The second pathway operates later, at oocyte stage III/IV, and is involved in germ layer formation by localization of Vg1 and VegT mRNAs (Kloc and Etkin 1995). At stage I and II of oogenesis these mRNAs are distributed throughout the oocyte cytoplasm, but are excluded from the MC. By late stage III, Vg1 and VegT mRNAs are actively transported to the vegetal hemisphere, where they become anchored (King et al. 2005). The germ cell specific mRNA of XDead end (XDE) represents an exception as it localizes via the late localizing pathway (Figure $1.3 A+B$, left part - oogenesis) (Horvay et al. 2006). Late localizing Vg1 and VegT mRNA are suggested to be bound already in the nucleus through the mRNA-binding proteins Vg1RBP, VgRBP60 and 40LoVe (Kress et al. 2004, Czaplinski et al. 2005) and exported to the cytoplasm. There, localization ribonucleoproteins (RNPs) are remodeled and cytoplasmic factors, like XStaufen, Proline-rich RNA-binding protein (Prrp) and ElrA/B1 assemble into the complex. XStaufen associates with kinesin and might play a role in motor-protein-recruitment. Finally, the RNP complex is transported by motor proteins along cytoskeletal elements to the vegetal cortex, where it is anchored (Figure 1.2) (Allison et al. 2004, Yoon and Mowry 2004, Arthur et al. 2009, Kroll et al. 2009). RNA localization in the oocyte depends on cis-acting sequences, so called localization elements (LE), mostly residing in the 3' untranslated region (UTR) of transcripts. These sequences are very diverse in length and mediate the interaction with localization factors during different steps of vegetal transport (Zhou and King 2004, Horvay et al. 2006). Statistical analysis of LEs identified a relatively common repeated motif of $4-6$ nucleotides to be required for proper mRNA-protein interaction that mediates mRNA localization. In Xenopus, these motifs often include CAC-repeats (Deshler et al. 1998, Betley et al. 2002). Interaction of LEs with proteins of the transport machinery leads to 
the assembly of RNP-complexes, required for proper localization and regulation of translation as well as stability of RNAs (Kloc et al. 2002, Bauermeister et al. 2014).

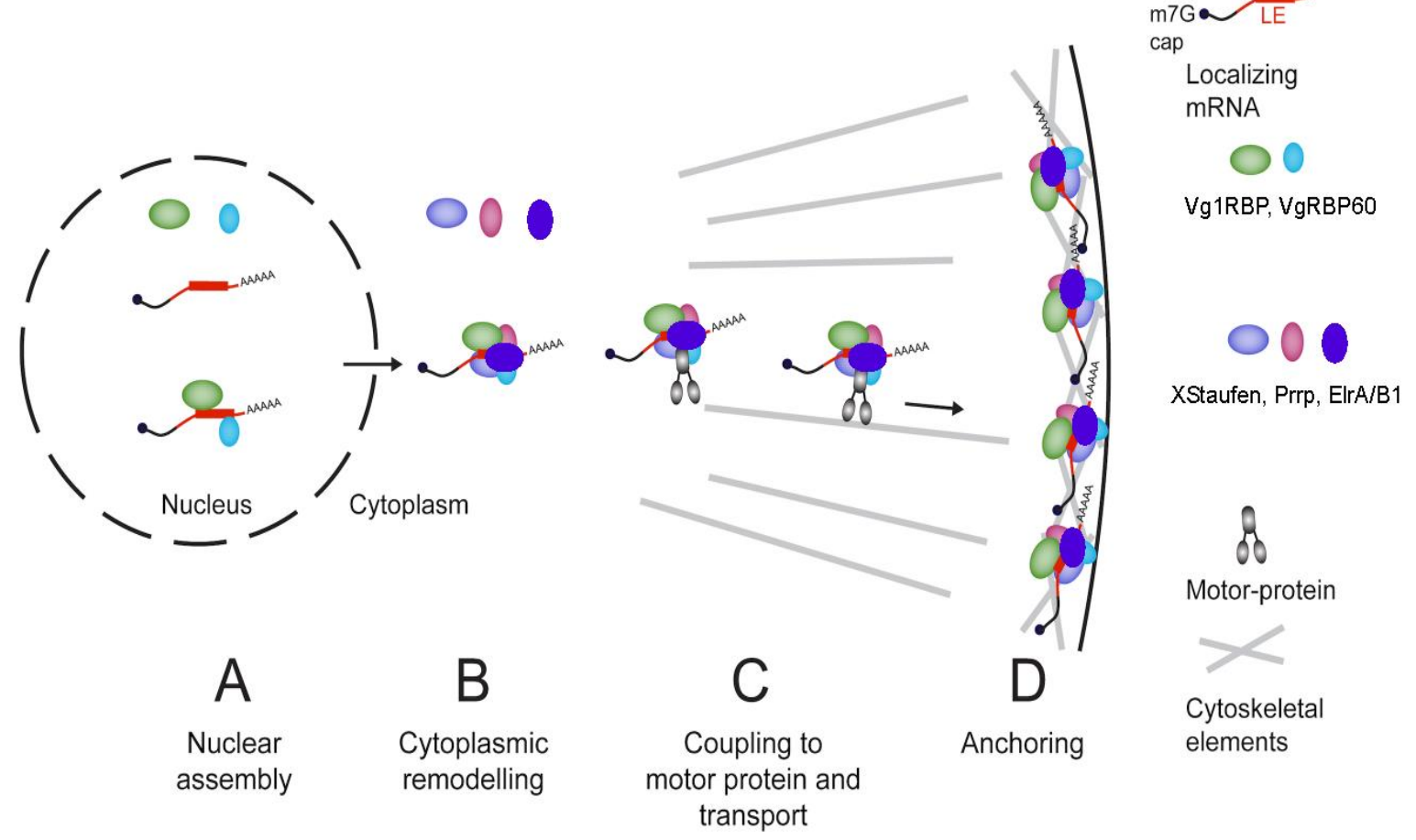

Figure 1.2. Model of vegetal mRNA localization in Xenopus oocytes.

A) In the nucleus the first set of localization factors (VgRBP60, Vg1RBP) bind to the LE of mRNAs.

B) After export to the cytoplasm, the RNP complex is remodeled and further proteins (XStaufen, Prrp, ElrA/B) associate with the complex.

C) The RNP is coupled to motor proteins and migrates along the cytoskeleton.

D) Anchoring of the transport complex occurs at the vegetal cortex.

(modified Bauermeister et al. 2014)

\subsubsection{Formation and development of germ cells in Xenopus embryos}

Inherited maternal components are enriched at the vegetal cortex of the egg and become asymmetrically distributed between daughter cells after fertilization. At blastula stage, three to seven cells, located between the vegetal pole and the base of the blastocoel, contain parts of the germ plasm. By the onset of gastrulation, germ plasm bearing cells are specified as PGCs. At the end of gastrulation, PGCs are positioned to the posterior endoderm and undergo the first mitotic divisions. At stage 25-26 first lateral-anterior and later dorsal movement of PGCs starts. By stage 40, germ cells migrate actively to the dorsal endodermal crest and are incorporated into the dorsal mesentery at stage 43-44 (Figure 1.3 A). Movement along the dorsal mesentery positions germ cells to the region of future gonads, which are formed by stage 50 (Whitington and Dixon 1975, Houston and King 2000b). 
A

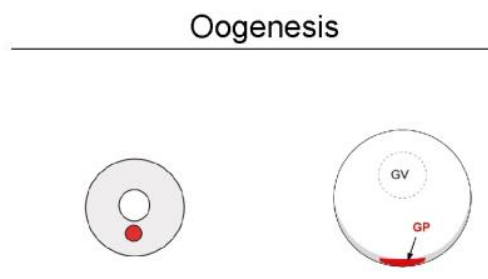

B

St.I

St.V

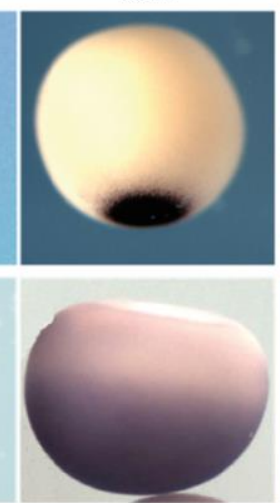

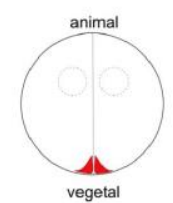

4-cell stage
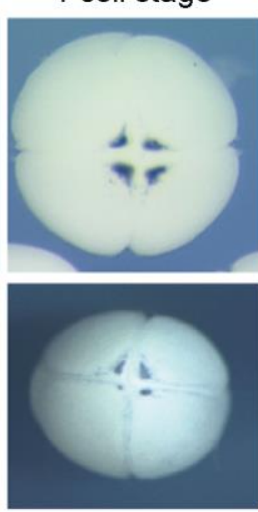

Embryogenesis

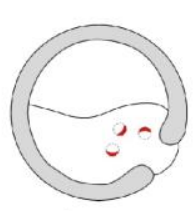

St.11

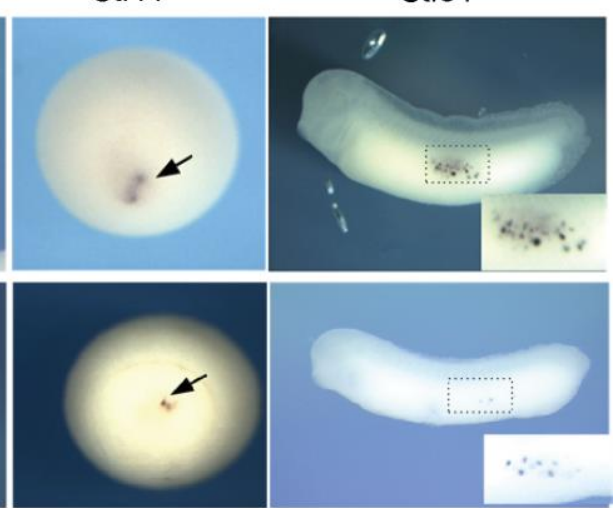

Figure 1.3. PGC development in $X$. laevis embryos.

A) Schematic view of germ cell development in $X$. laevis. In stage I oocytes germ cell specific mRNAs (red), except $X D$ ead end $(X D E)$, are associated with the mitochondrial cloud (MC) and become localized to the vegetal cortex at stage IV-V, where they are anchored and form the germ plasm (GP, red) together with other transport factors. During early cleavage stages, the germ plasm clusters in granular islands at the vegetal pole. By blastula stage, the germ plasm is associated with plasma membranes and is inherited via asymmetrical cell division. During gastrulation (St. 11), the germ plasm translocates to the peri-nuclear region of specified germ cells, which are positioned at the posterior endoderm by gastrulation movements. There they undergo the first mitotic divisions. At stage 24 active migration of PGCs starts and they migrate laterally (St. 31) and then dorsally to reach the dorsal crest.

B) Localization of Xpat and XDE mRNA during oogenesis and embryogenesis. Whole mount in situ hybridization (WMISH), showed association of Xpat mRNA with the MC in stage I oocytes and the anchoring at the vegetal cortex at stage IV-V. During early embryogenesis the Xpat mRNA colocalizes with the germ plasm in the dividing blastomeres and becomes germ cell specific in later stages.

$X D E$ is a late localizing mRNA, thus present in the whole cytoplasm omitting the mitochondrial cloud in stage I oocytes. In stage IV-V oocytes, XDE transcripts accumulate at the vegetal pole. In the embryo XDE mRNA colocalizes with the germ plasm and becomes PGC specific in later stages of embryogenesis.

(modified from Houston and King 2000, Horvay 2006, Koebernick 2010)

Besides the proteins XDE and XDazl, which will be discussed in section 1.5 and 1.7, Xcat2 protein plays an important role in Xenopus germ cell development. Xcat2 mRNA is restricted to the germ plasm, where it is first translated after fertilization (Lai et al. 2011, Lai et al. 2012). Inhibition of Xcat2 translation leads to failure of PGC migration, followed by their loss through apoptosis (Lai et al. 2012). Interestingly, Xcat2 is also required to repress translation of somatic genes in PGCs in association with Pum2 by blocking phosphorylation of the C-terminal domain of RNA pol II. Thus, germ cells in the early blastula and gastrula require Xcat2 for migration, survival and to preserve PGC fate (Lai et al. 2012). 


\subsection{Maternal to zygotic transition and miR-mediated decay in Xenopus and zebrafish}

Early embryonic development depends on the inheritance of maternal mRNAs and proteins loaded into the oocyte (Tadros and Lipshitz 2009). At mid-blastula transition (MBT), developmental control transits from dominance of maternal components to zygotically encoded determinants. MBT is defined by an increase in cell cycle length through gap phases and desynchronized mitosis. It occurs around stage 8.5-9 in Xenopus and between the 64-cell and 256-cell stages in zebrafish (Yasuda and Schubiger 1992, Lee et al. 2014). During the subsequent phase, referred to as maternal to zygotic transition (MZT), interference of pre-existing maternal mRNAs with zygotic transcripts is prevented (Giraldez 2010). Clearance of maternal determinants is accomplished by two activities: the first is maternally encoded while the second one requires zygotic transcription. Within hours after fertilization, a large portion of maternal mRNAs is eliminated (Figure 1.4) (Schier 2007).

In Xenopus, the maternally provided EDEN-binding protein (EDEN-BP) mediates the first wave of translational silencing by targeting mRNAs bearing an embryonic deadenylation element (EDEN) within their 3' UTR (Paillard and Osborne 2003). A second wave of clearing maternal mRNAs is initiated by zygotic transcription of microRNAs (miRs) (Figure 1.4). Zygotic transcription of somatic mRNAs starts at MZT. Nevertheless, germ cell specific transcripts are still silenced during that time. Their transcription begins in a second wave of zygotic transcription (Figure 1.4) (Lai and King 2013).

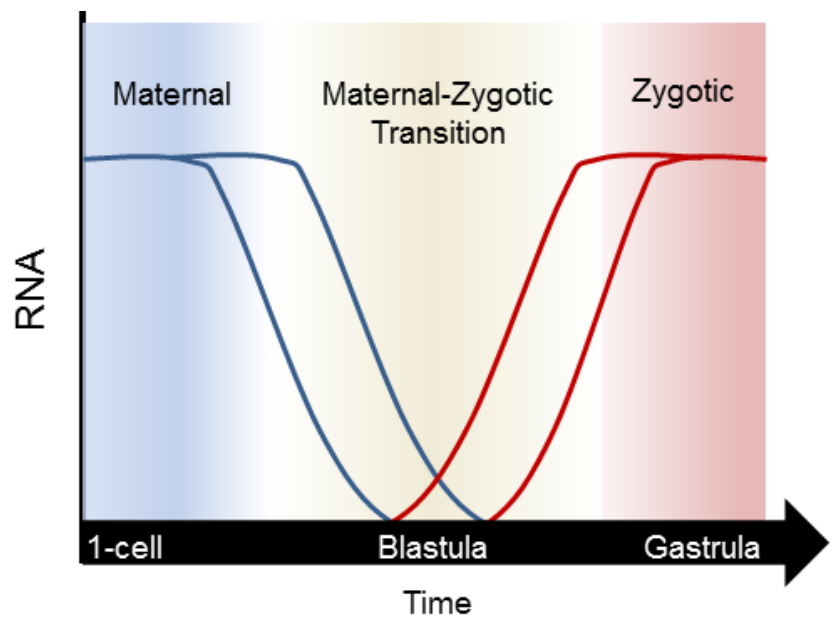

Figure 1.4. MiR-mediated mRNA decay of maternal transcripts at maternal-to-zygotic transition (MZT). Maternal mRNAs (blue) drive the early development in absence of zygotic transcription. Further, maternally supplied proteins can silence transcripts (first blue wave). Zygotic gene expression starts at blastula stage 8.5 in Xenopus and leads to the expression of zygotic RNAs, including miRs (first red wave) leading to a second wave of maternal mRNA decay (second blue wave). The process of zygotic gene activation and maternal transcript degradation is defined as maternal-to-zygotic transition (MZT). Germ cells are transcriptional silenced during $M Z T$, expression of PGC transcripts starts in a second wave of zygotic transcription (second red wave). (modified from Schier 2007) 
MiRs are small $\sim 22 \mathrm{nt}$ long RNAs, which regulate gene expression post-transcriptionally by base-pairing to target mRNAs (Bartel 2004, 2009). Usually they form imperfect hybrids with sequences in the 3' UTR of target mRNAs, thereby a miR 5'-proximal "seed" sequence (positions 2-8) provides most of the pairing specificity (Fabian et al. 2010). MiRs are mainly transcribed by polymerase II from intronic sequences of potential gene targets (Tang and Maxwell 2008). Maturation of miRs is achieved by processing of longer double-stranded RNA precursors (pri-miR) into pre-miRs by Drosha and DGCR8 in the nucleus (Figure 1.5). These pre-miR hairpin structures are then transported to the cytoplasm and further processed through sequential cleavage by Dicer into double stranded intermediates, which are incorporated into a silencing complex (miRISC). However, only one strand will be selected for loading onto an Argonaute (Ago2) protein in order to guide the complex to the respective target mRNA, resulting in their deadenylation, repression and decay (Figure 1.5) (Winter et al. 2009, Treiber et al. 2012).

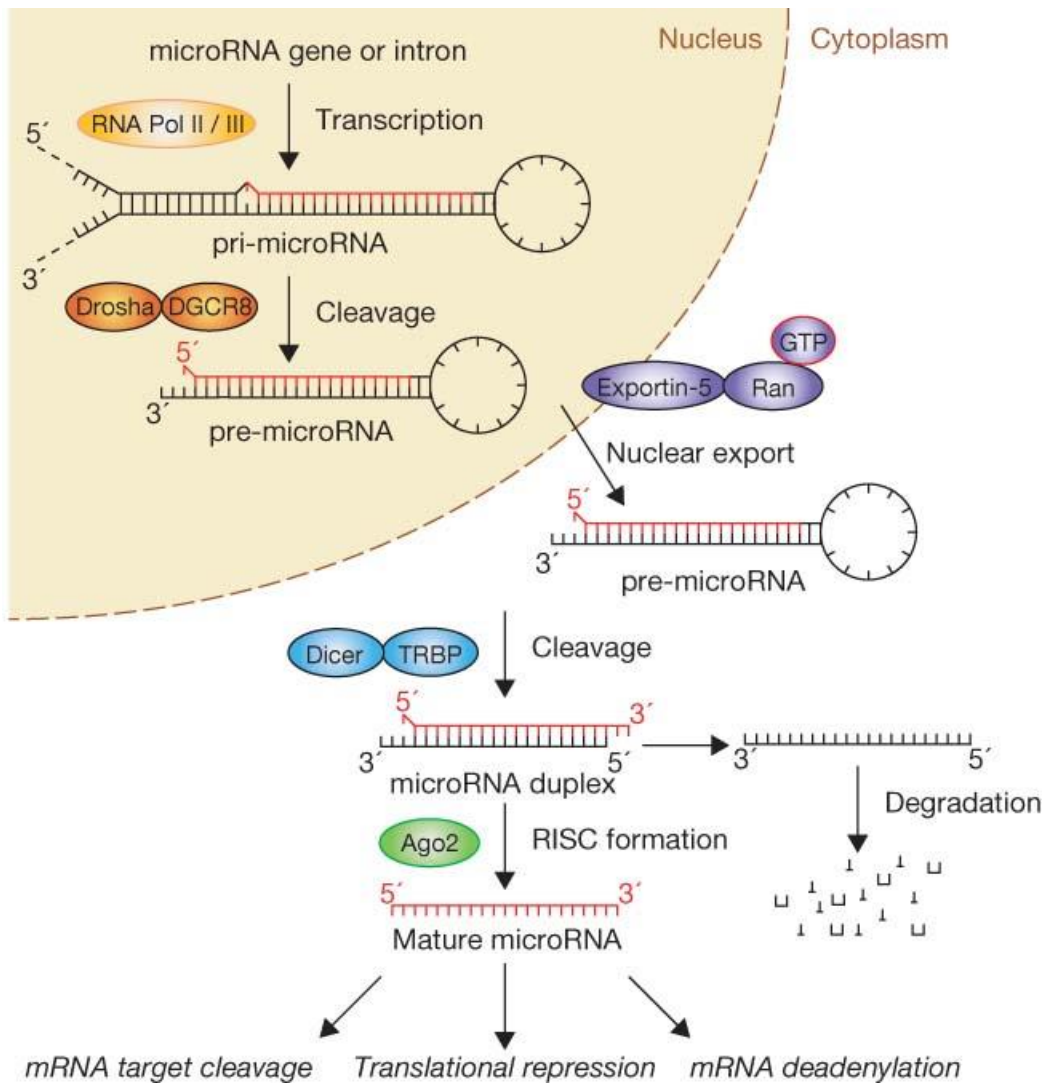

Figure 1.5. Mechanism of microRNA (miR) processing.

In the nucleus, pri-microRNA is transcribed by RNA polymerase II and processed into pre-microRNA by Drosha/DGCR8. The resulting precursor hairpin is exported from the nucleus by Exportin-5-Ran-GTP. In the cytoplasm, the RNase Dicer in complex with the double-stranded RNA-binding protein TRBP cleaves the premicroRNA hairpin to its mature length. The functional strand of the mature microRNA is loaded together with Argonaute (Ago2) proteins into the RNA-induced silencing complex (RISC). This complex is guided by the microRNA to target mRNAs leading to silencing through mRNA cleavage, translational repression or deadenylation, whereas the passenger strand (black) is degraded. 
To date, many functional analyses have shown that miRs not only control temporal gene expression, but also modulate transcript levels in specific regions of the zygote (Bagga et al. 2005, Mishima et al. 2009). Inhibition of zygotic transcription was shown to result in stabilization of a large portion of maternal mRNAs (Newport and Kirschner 1982, Lund et al. 2011). However, the factors responsible for the selective and active degradation are still unknown.

The orthologous miRs, miR-430 in zebrafish and miR-427 in Xenopus, are abundantly expressed during maternal-to-zygotic transition. In zebrafish, mutation of the Dicer protein led to the identification of more than 200 mRNAs, which are targeted by miR-430 in vivo. The degradation of these mRNAs occurs by miR-430 mediated deadenylation (Giraldez et al. 2006). In Xenopus embryos, the target list of the ortholog miR-427 is not complete until now, as genome-wide analyses are still elusive. Lund and colleagues provided evidence that miR-427 directly promotes deadenylation of maternal Cyclin $A 1$ and B2 mRNAs (Lund et al. 2009).

\subsection{Germ cell specific RNA protection from miR-mediated decay}

In somatic cells, zygotic miR transcription ensures the degradation of maternally supplied mRNAs after MBT. Germ cells are transcriptionally silent during that time (Venkatarama et al. 2010). Therefore, germ line transcripts need to be protected against miR-mediated decay by a maternally provided mechanism during MZT. Recent studies provided interesting insights into how RNA-binding proteins can modulate miR promoted regulation depending on cell type and cellular state. Mishima et al. reported that miR-430 targets the 3' UTR of nanos1 during zebrafish embryogenesis in the soma. Interestingly, this degradation does not occur in PGCs. Most probably, PGCs promote the expression of nanos 1 and other germline-specific mRNAs by recruiting specific factors to the 3' UTR that increase stability or translation (Mishima et al. 2006). This hypothesis was supported by the finding that the RNA binding protein Dead end (DND1) protects nanos1, tudordomain-containing-protein 7 (tdrd-7) and huB mRNA from miR-430 mediated clearance in zebrafish germ cells. It is supposed that the DND1 binding site in the nanos 1 3' UTR overlaps with the miR-430 target site, such that DND1 could provide steric protection from miR-430 binding (Kedde et al. 2007, Mickoleit et al. 2011). A similar mechanism might operate in human cell lines, where DND1 protects p27 mRNA from miR-221 targeting (Figure 1.6) (Ali et al. 2013). 
In the zebrafish germ line, a second protein, Dazl (Deleted in azoospermia-like), was identified to protect $h u B$ and $t d r d-7$ from miR-430 mediated clearing through a different mechanism than DND1 (Takeda et al. 2009, Wiszniak et al. 2011). Dazl antagonizes miR-430 activity by inducing mRNA polyadenylation and thus increasing translation efficiency, when bound to the tdrd7 3' UTR in zebrafish (Figure 1.6). Takeda and colleagues identified a stabilizing effect of Dazl protein on its own mRNA (Takeda et al. 2009). From these findings it can be suggested that Dazl might also enhance the expression of other germ cell-specific mRNAs (Pauli et al. 2011).

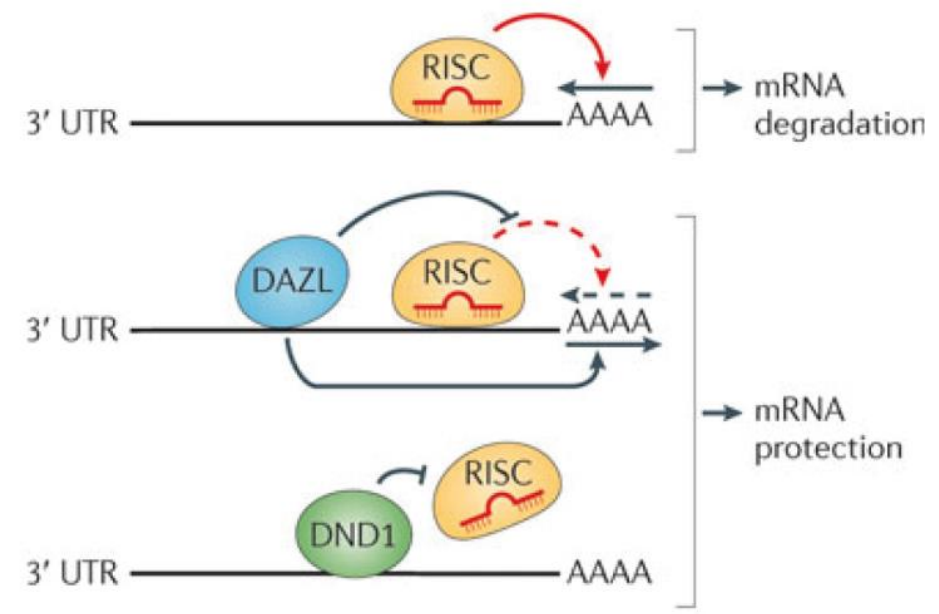

Figure 1.6. Modulation of miR-mediated repression in germ cells by the RNA-binding proteins DND1 and Dazl.

The model depicts post-transcriptional regulation of mRNA targets by miRs. In the absence of a protective mRNA binding protein the miR induced silencing complex (miRISC) is recruited to the $3^{\prime}$ UTR of its target mRNA and accelerates deadenylation of the poly $(A)$ tail, leading to degradation of the mRNA. Dazl binding to the 3' UTR of its target mRNA antagonizes miR-mediated repression by enhancing polyadenylation.

Binding of DND1 to the cis-elements within 3' UTRs of specific mRNAs blocks miR target recognition of the miRISC and thus antagonizes miR-mediated repression of mRNAs in germ cells (from Pauli et al. 2011).

\subsection{Dead end}

Xenopus Dead end $(X D E)$ represents a germ plasm specific transcript, which is the ortholog to the zebrafish dead end (DND1) described above (Horvay et al. 2006, Kedde et al. 2007). During Xenopus oogenesis, XDE mRNA localizes to the vegetal pole via the late transport pathway. Nevertheless, it becomes germ cell specific during embryonic development. This localization is achieved by binding of a set of transport proteins, including ElrA/B proteins, to the localization element within the 3' UTR of XDE, (Horvay et al. 2006, Arthur et al. 2009). Koebernick and colleagues could show that the somatic clearance of $X D E$ mRNA is caused by miR-18 targeting. Xenopus ElrB1 protein, which is a component of the vegetal localization complex, can counteract miR-18 mediated mRNA clearance together with the XDE protein itself (Koebernick et al. 2010). The 
XDead end transcript encodes a 354 aa long RNA-binding protein with one RNArecognition motif (RRM), which binds trim36 mRNA and anchors it at the vegetal cortex in Xenopus oocytes. Knockdown of maternal XDE disrupts the vegetal cortical microtubule assembly, which is necessary for the translocation of determinants such as wnt11 to the dorsal site. Translational inhibition of XDead end in the embryo leads to clustering and loss of PGCs at tadpole stage (Horvay et al. 2006, Mei et al. 2013). These findings indicate a requirement of XDE for germ cell migration and survival as well as Xenopus axis specification.

The zebrafish Dead end RRM was shown to be important for targeting protein expression to the germ cells and to the perinuclear granules. To fulfill this function the protein translocates between germ cell nucleus and germ cell granules (Slanchev et al. 2009). In mouse, the Dead end 1 gene (called Ter) is also expressed in PGCs and a mutation within the Ter gene locus increases the incidence of testicular germ cell tumors (teratomas) (Matin 2007).

\section{$1.6 \mathrm{Hu}$ proteins (ElrA and ElrB1)}

ELAV/Hu proteins belong to a highly conserved family of RNA binding proteins (RBPs) consisting of four family members. The proteins $\mathrm{HuB}, \mathrm{HuC}$ and $\mathrm{HuD}$ are predominantly neuron-specific and cytoplasmic, while $\mathrm{HuA}(\mathrm{HuR})$ is ubiquitously expressed in nuclei. A common characteristic among all $\mathrm{Hu}$ proteins is the presence of three RRMs and a flexible hinge/linker region between RRM2 and RRM3 allowing shuttling between nucleus and cytoplasm (Good 1995, Fan and Steitz 1998) (Figure 1.7). Neuronal Hu proteins were originally identified as specific tumor antigens in lung cancer patients (Keene 1999). Hu proteins bind directly to AU-rich elements (ARE) within the 3' UTR of target mRNAs and relieve repression of miR-mediated decay, thereby stabilizing and activating translation of mRNAs (Jain et al. 1997, Bhattacharyya et al. 2006, Koebernick et al. 2010). Moreover, a function of Hu proteins in RNA-processing during RNA-splicing and polyadenylation is described (Srikantan et al. 2012). The orthologous Drosophila ELAV genes were originally identified in mutants showing an embryonic lethal abnormal visual (ELAV) phenotype (Campos et al. 1985).

Interestingly, HuD protein was found to bind directly to its own 3' UTR and thus, regulate its posttranscriptional expression (Abe et al. 1996). Recently, zebrafish Dazl was shown to specifically bind the $H u B 3^{\prime}$ UTR and protect the message from degradation. Thereby, Dazl enhances $H u B$ translation, leading to the germ cell specific expression of $H u B$ protein (Wiszniak et al. 2011). 
In Xenopus, HuA (ElrA) and HuB (ElrB1) were shown to be involved in the vegetal localization process in oocytes (Arthur et al. 2009). The hinge region in ElrB1 was described to be responsible for oligomerization of ElrB1 proteins in presence of the $\mathrm{Vg} 1$ Translational element (VTE) RNA (Devaux et al. 2006). In contrast, ElrA proteins do not oligomerize. Furthermore, ElrB1 is found in the brain, testis and ovary, while ElrC (HuC) and ElrD (HuD) are exclusively expressed in the brain. ElrA is expressed ubiquitously in all tissues with relatively high mRNA levels in the ovary. ElrB1 displays a complex expression pattern. Neural-specific transcripts are present late in development, by tadpole stage 44, whereas ovary-specific ElrB1 mRNA is already present in the early embryo by gastrula stage. The expression pattern indicate a potential role of ElrB1 in germ cell formation and early development in addition to neuronal functions (Good 1995).

\begin{tabular}{l|l|l|l|l|l|} 
RRM1 & & RRM 2 & hinge region & RRM 3 \\
\hline
\end{tabular}

Figure 1.7. Schematic depiction of the $\mathbf{3 8 9}$ aa long ElrB1 protein containing three RRMs

RRM1 and 2 are responsible for the detection of AU-rich regions, whereas the RRM3 interacts with the poly $(A)$ tail of mRNAs or other proteins. Furthermore, RRM3 can enhance the binding of RRM1. The hinge region between RRM2 and RRM3 mediates shuttling between nucleus and cytoplasm (according to Fan 1998).

\subsection{Deleted in Azoospermia-like (Dazl)}

Dazl belongs to a family of RNA binding proteins, called the DAZ family, which mediates important roles in gametogenesis.

\subsubsection{Origin and structure of the DAZ family genes}

The human Deleted in azoospermia (DAZ) is located within the azoospermia factor locus (AZFc) on the Y-chromosome and was first identified in 5-10\% of infertile men displaying diverse azoospermia and oligospermia defects (Reijo et al. 1995b, Reynolds and Cooke 2005). Further studies on proteins located in the AZF locus identified the 'RNA binding motif, Y-chromosome' (RBMY) and DAZ proteins as potential cause of the genetic defects (Foresta et al. 2002). RBMY is believed to play a role in splicing. DAZ was identified in patients suffering from spermatogenic failure exhibiting microdeletions of the AZF region. Both proteins, RBMY and DAZ, are present in multiple copies within the AZF region (Ma et al. 1993, Reijo et al. 1995b, Elliott et al. 1997, Yen et al. 1997). The DAZ family consists of three family members: $D A Z, D A Z$-like and BOULE being expressed 
exclusively in germ cells and encoding for RNA binding proteins with a unique DAZ domain/repeat of 24 amino acid residues (Figure 1.8). DAZ repeats have been described to mediate protein-protein interaction as well as polysome association. BOULE and DAZL exhibit only one DAZ repeat and are autosomal single-copies, whereas the DAZ gene encodes a protein containing multiple DAZ repeats arranged in tandems and is located on the Y-chromosome (Yen 2004). These three proteins are present in many different species ranging from invertebrates to humans (Reijo et al. 1995a, Haag 2001, $\mathrm{Xu}$ et al. 2001). However, DAZ is only found on the Y-chromosome of humans and old world monkeys, while invertebrates encode boule (Brook et al. 2009). BOULE orthologs have been identified in $C$. elegans, Drosophila, mice and humans. Thus, BOULE is considered to be the ancestor of the DAZ family evolved by transposition, repeated amplification and reduction. Orthologs of $D A Z L$ are more closely related to $D A Z$ than to BOULE and were found in vertebrates only (Yen 2004). Although DAZ, DAZL and BOULE belong to the same family, their conservation is restricted to the RRM and the DAZ domain that share 78 and $50 \%$ sequence similarity, respectively (Figure 1.8) (Brook et al. 2009).
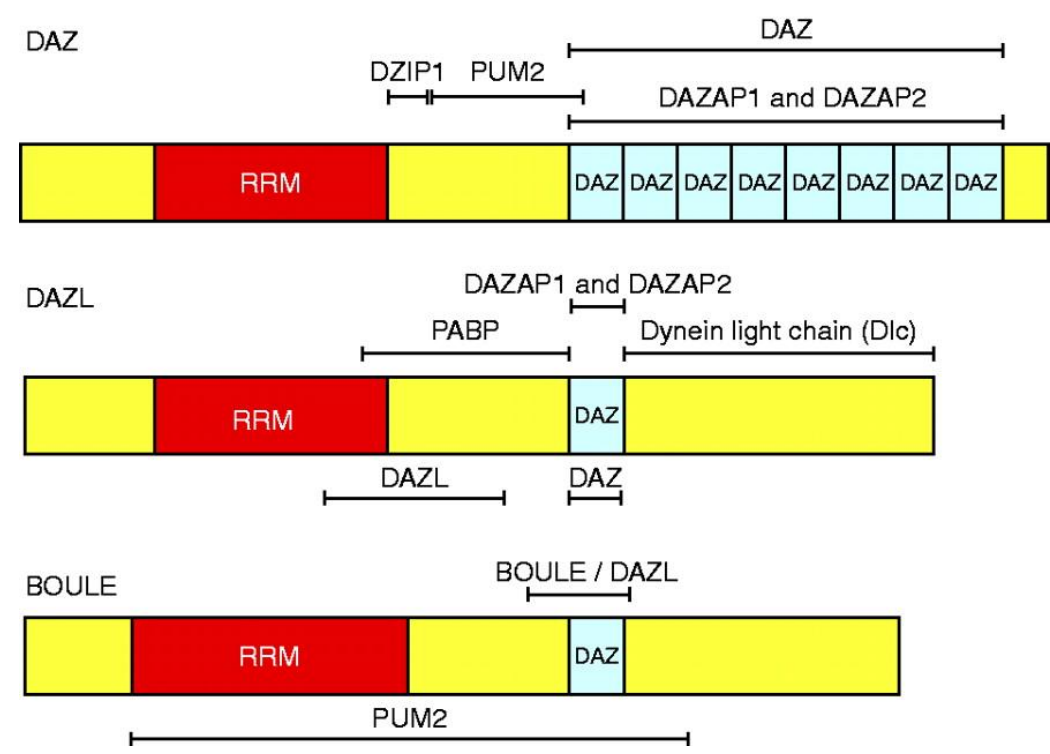

Figure 1.8. Structure and binding partner of DAZ family proteins.

Schematic view on the protein domain structures and interaction partners of the DAZ family members: $D A Z$, DAZL and BOULE. All three proteins contain two binding motifs, a single RRM and the characteristic DAZ domain. The DAZ gene can exhibit 7 up to 24 copies of the DAZ domain (Brook et al. 2009).

In men, most often four DAZ gene copies are found coding for proteins with three RRMs and seven to 24 DAZ repeats. As depicted in Figure 1.8, the DAZ family members contain a high variation in the number of exhibited DAZ domains, indicating a binding of these proteins to different interaction partners and no functional redundancy. Indeed, null 
mutations of the human $D A Z$ or mouse Dazl gene cause defects during spermatogenesis, although other family members are present (Yen 2004). Interestingly, phenotypic rescue experiments revealed a contribution of different DAZ-family proteins to gametogenesis by related molecular mechanisms. Thus, ectopic expression of XDazl and hBoule in Drosophila or human DAZ expression in mice can partially rescue the mBoule and mDazl knock out phenotypes (Houston et al. 1998, Slee et al. 1999, Xu et al. 2003).

\subsubsection{Developmental expression of Dazl}

Although DAZ family proteins are mainly expressed in germ cells, their expression patterns are more complex. In mice, Dazl (mDazl) mRNA was detected in testis and ovary. Immunostaining revealed mDazl protein presence in spermatogonia and early and late spermatocytes (Reijo et al. 1996, Ruggiu et al. 1997). Human DAZL transcripts were detected in testes and ovary isolated from human fetuses at approximately 23 weeks of gestation. Later in development, the mRNA was found in oogonia, oocytes and primordial follicles in the ovary as well as in germ cells in the testis (Brekhman et al. 2000). Moreover, DAZL transcripts are expressed predominantly in the cytoplasm of spermatocytes and spermatids in the testis (Reijo et al. 2000, Tung et al. 2006).

Xenopus Dazl (XDazl) mRNA localizes to the vegetal cortex of oocytes, becomes PGC specific in the early embryo and is detectable in spermatogonia as well as spermatocytes in the testis (Houston et al. 1998). Mita and colleagues could detect the presence of the protein during early developmental stages by Western Blotting. However it disappears after gastrula stage 12-13. As soon as migrating germ cells have reached the genital ridge the XDazl protein reappears in the germ cells. Immunofluorescence staining identified the XDazl protein presence in all stages of male and female germ cells except mature spermatozoa. (Mita and Yamashita 2000). Zebrafish Dazl (zDazl) is expressed in gonads of both sexes. The mRNA is maternally provided and localizes to the vegetal cortex in oocytes. At the onset of embryogenesis, maternal $z D a z / m R N A$ was detected at the vegetal pole. In male, zDazl transcripts were detectable in spermatogonia and primary spermatocytes, but not in secondary spermatocytes, spermatids or mature sperm. However, in invertebrates the DAZ family proteins are not restricted to germ cells and gonads, as Boule is expressed in Drosophila larval neurons and adult brain (Brook et al. 2009). 


\subsubsection{Requirement of Dazl in germ cell development}

DAZL is widely involved in germ cell development and defects in DAZL function are implicated in human infertility (Reynolds and Cooke 2005). In mice, Dazl knockout leads to loss of germ cells and a complete absence of gametes in both sexes. Female Dazl null mice develop normal until day 15 post coitum, but show a decrease in oocyte number by day 19 post coitum. Furthermore, the formation of germ cells and follicles is disturbed (Saunders et al. 2003). Males lacking Dazl display a reduction of PGCs at late prenatal stage and postnatal testes show very low germ cell numbers, which arrest before meiosis (Ruggiu et al. 1997, Schrans-Stassen et al. 2001). In accordance to these findings, Dazl was described as an intrinsic factor required for germ cell entry into meiosis (Lin et al. 2008). In Drosophila, male boule mutants are sterile and their spermatocytes are arrested at the G2/M transition stage of meiosis, indicating a function in spermatogenesis (Eberhart et al. 1996).

Maternal depletion of XDazl transcripts in Xenopus resulted in defects in PGC formation and migration, although early germ plasm structure and localization were unaffected. XDazl-deleted embryos show normal PGC formation in early embryonic development, but they fail to migrate to the dorsal endoderm and developing embryos lack germline progenitors at late tailbud stage. Most probably the PGCs undergo apoptosis or differentiate into somatic cells (Houston and King 2000a). However, the lack of zygotically expressed PGC markers, like Xpat, makes it difficult to evaluate the state of PGC specification in these cells. Based on these findings, it can be concluded that Xenopus Dazl functions in the development of PGCs, in their differentiation, migration and maturation to gametes (Houston and King 2000a).

\subsubsection{Dazl as a translational regulator}

A common characteristic of all DAZ family members is the RRM domain and its primarily cytoplasmic localization. The potential of the Dazl protein to bind mRNAs and its presence in germ cells argues for a potential role in gene regulation there (Houston et al. 1998, Houston and King 2000a). As Dazl loss-of-function leads to defects in oogenesis and spermatogenesis, it is most likely that mRNAs targeted by Dazl encode proteins with functions in these two mechanisms (Brook et al. 2009). In mouse and zebrafish, Dazl was found on actively translating polysomes from testis, suggesting a function in mRNA regulation and stability (Tsui et al. 2000a, Maegawa et al. 2002). The important role of Dazl to stimulate translation of mRNA targets was shown by their upregulation in an in 
vitro translation assay and by tethering the protein to the 3' UTR of reporter mRNAs in Xenopus oocytes (Maegawa et al. 2002, Collier et al. 2005). In zebrafish and mouse the Dazl binding motif was defined as $\mathrm{U}_{2-10}[\mathrm{G} / \mathrm{C}] \mathrm{U}_{2-10}$ located in the 3' UTR of target mRNAs (Maegawa et al. 2002, Reynolds et al. 2005). Mutation of this mRNA target motif abolishes Dazl binding. Furthermore, the RRM mediates binding to the target mRNA (Tsui et al. 2000b). Interestingly, a single point mutation in the zebrafish RRM, F91A (equivalent to $\mathrm{F} 84$ in $\mathrm{mDazl}$ and $\mathrm{hDAZL}$ ), was sufficient to prohibit mRNA binding, confirming that the RRM is essential for mRNA recognition by Dazl (Maegawa et al. 2002). The characteristic RNP1 (8 aa) and RNP2 (6 aa) motifs contain aromatic residues that are frequently involved in recognition of nucleic acids and are identical between $\mathrm{mDazl} / \mathrm{hDAZL}$ and the human DAZ proteins. Crystal structure analysis of the RRM from murine Dazl (mDazl) reveals high sequence-specific recognition of a GUU triplet by a pair of $\beta$-strands, alone and in complex with mRNA sequences from the $3^{\prime}$ UTRs of mouse vasa homolog (Mvh) (Jenkins et al. 2011). Moreover, tethering of multiple Dazl molecules to the mRNA enhances translation, indicating the presence of many Dazl binding sites, which might be required for translational activation (Collier et al. 2005). In accordance with this suggestion, Reynold and colleagues identified target mRNAs with multiple Dazl- binding motifs (Reynolds et al. 2005). A recent study indicated that zDazl binding to multiple GUU-repeats in the 3' UTR of the huB mRNA can enhance translation (Wiszniak et al. 2011). Furthermore, Mvh and Sycp3 mRNA were described as Dazl targets. Both encode for proteins involved in progression of prophase I in meiosis. The male phenotypes of Mvh and Sycp3 null mice are similar to those of Dazl null mice (Tanaka et al. 2000, Yuan et al. 2000, Reynolds et al. 2005). In addition, ectopic expression of Dazl in embryonic stem cells (ESCs) led to their differentiation towards mature functional gametes (Yu et al. 2009). A recent study identified a murine Dazl splice variant as negative translational regulator of $M v h$, as well as Oct3/4 and Sox2 in ESCs (Xu et al. 2013). Both, Oct3/4 and Sox, are well known pluripotent-specific transcription factors. By contrast, $M v h$ is necessary for male germ cell development. Therefore, a role of Dazl in balancing pluripotency and germ cell traits of ESCs to allow PGC differentiation can be suggested. Besides, Dazl may function in maintaining the pluripotency by inhibiting translation of germ cell markers to avoid spontaneous unwanted PGC specification (Smorag et al. 2014). Analyses designed to identify human Dazl target mRNAs showed regulation of CDC25 and severe polyadenylation of actin 1 (SDAD1), which are cell cycle regulators (Brook et al. 2009).

In Xenopus, many mRNAs with short poly $(\mathrm{A})$ tails are stored during oogenesis and 
spermatogenesis. The Ringo/Spy mRNA was shown to be bound by XDazl in oocytes. This mRNA activates CDK2, which initiates a phosphorylation cascade responsible for oocyte maturation. Nevertheless, in prophase I-arrested oocytes Ringo/Spy mRNA is translationally repressed by a complex composed of XDazl, Pumilio 2 (Pum2) and embryonic Poly-A-binding protein (ePABP) proteins. In the suggested model, Pum2 and XDazl directly bind to the 3' UTR of its target mRNA and recruit the ePABP. EPABP, in turn, interacts with 5 ' end-associated factors to enhance the assembly of a closed-loop RNP conformation. This structure abolishes the interaction of ePABP with factors of the translation initiation machinery, such as elF4G and elF4E. Following oocyte stimulation with progesterone Pum2 breaks away from the RNP, leading to the recruitment of eIF4G and elF4E and activation of mRNA translation (Figure 1.9) (Collier et al. 2005, Padmanabhan and Richter 2006, Bettegowda and Smith 2007).
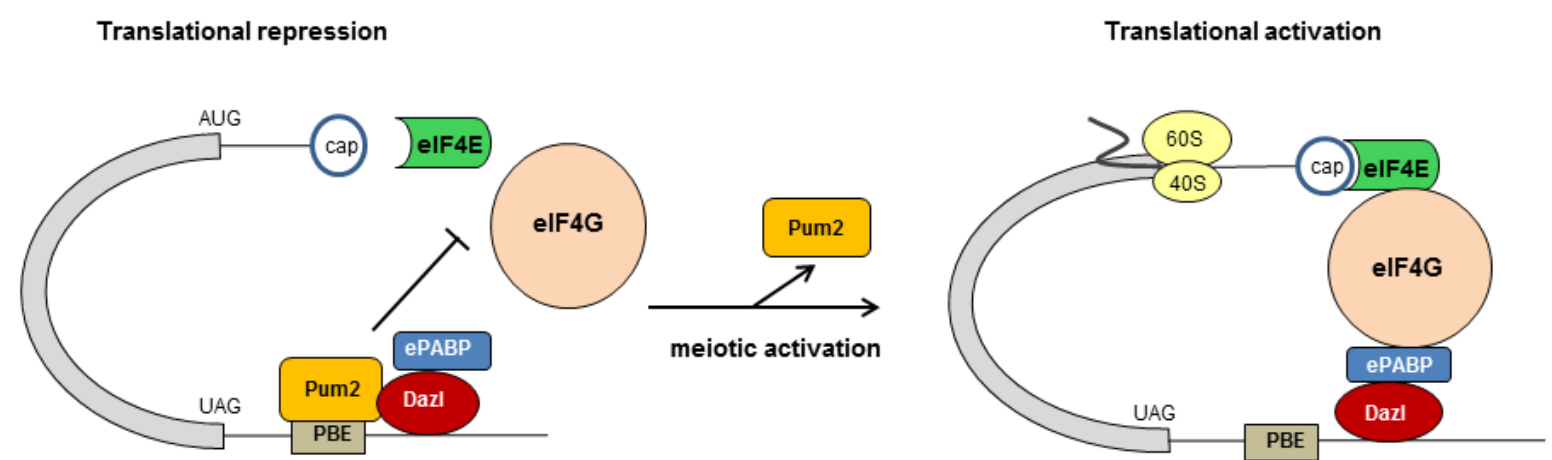

Figure 1.9. Model of Dazl mediated translational regulation of target mRNAs during oocyte maturation. Pum2 represses together with Dazl, which is bound to ePABP, its target mRNA by interacting with the Poly-Abinding element (PBE) within the 3' UTR. This complex formation interferes with the recruitment of translation initiation factors (e.g. elF4G and elF4E) to the 5' cap leading to abolished translation. Following oocyte maturation, Pum2 dissociates from the RNP and the Dazl/ePABP complex mediates binding of translation initiation factors to the translation start site resulting in activation of protein synthesis (after Padmanabhan, 2006 and Bettegowda, 2007).

Approaches using recombinant mDazl protein in the 'specific nucleic acids associated with proteins' (SNAAP) technique identified TSSK2, which encodes a testis-specific serine/threonine kinase, as Dazl target mRNA (Zeng et al. 2008). Moreover, Tex19.1 mRNA, encoding a protein implicated in gametogenesis, was described as Dazl target (Zeng et al. 2009). In addition, Dazl was shown to associate with stress granules in murine male germ cells upon heat-induced stress. Under these conditions, Dazl sequesters Rack1 mRNA, a proapoptotic factor, to the stress granules leading to blockage of the apoptotic MAPK pathway (Kim et al. 2012). 


\subsubsection{Dazl interacting proteins}

The depicted model of the DAZ family members in Figure 1.8 already indicates that DAZ proteins might interact with themselves and each other, suggesting a presence as homoor heterodimer in cells. As described above, one of the interaction partners of XDazl is Pum2, which was found to be responsible for translational regression of Ringo/Spy mRNA (Padmanabhan and Richter 2006). Furthermore, Dazl binds in vitro to the dynein light chain, a component of the motor complex responsible for vegetal mRNA localization in the oocyte (Lee et al. 2006). Dai and colleagues identified binding of Dazl to the mRNA-binding protein Dazl-associated protein1 (DAZAP-1, known as Prrp in Xenopus), which is widely expressed in human and mouse and was detected in high levels in testis. In the same study DAZAP-1 was found to bind to the DAZ domain, the motif which is responsible for polysomal localization of Dazl, indicating a function in Dazl-activated translation (Dai et al. 2001). The Xenopus ortholog Prrp was shown to be involved in vegetal transport of $V g 1$ and VegT mRNA (Zhao et al. 2001). Analyses of DAZAP-1 null mice revealed a function of the protein in spermatogenesis (Hsu et al. 2008). Further Dazl binding partners are DAZAP-2 and DZIP1, 2 and 3. However, little is known about their function with Dazl (Brook et al. 2009).

Taken together, Dazl has been presented as positive regulator of translation, most probably by binding to poly-(A)-binding proteins during translational initiation. Additionally, Dazl forms a complex on its target mRNA with the Pum2 protein suggesting that Dazl may have dual roles in both, activating germline genes and repressing somatic genes, in PGCs regulated by interactions with different proteins (Collier et al. 2005, Padmanabhan and Richter 2006, Reynolds et al. 2007). 


\subsection{Aim of the study}

With the onset of zygotic transcription maternally provided mRNAs are degraded by miRmediated decay. Interestingly, germ cell specific mRNAs are selectively protected against degradation, most probably due to binding of specific proteins. An interesting candidate, which could provide this protection, is Dazl as it is known to be involved in germ cell development and translational regulation.

The aim of the thesis is to investigate the function of XDazl protein in the germ cell specific mRNA protection phenomenon in Xenopus laevis embryos. Gain-of-function and loss-of-function experiments will be used to define the role of XDazl in PGC development. Furthermore, it remains to be determined whether XDazl cooperates with other proteins in order to exert its regulatory activities such on the level of translation. In order to analyze a possible connection between XDazl binding and protection against $\mathrm{miR}$ targeting in the mRNA, in vivo and in vitro experiments will be performed. Moreover, a potential supposed earlier function of XDazl during vegetal localization in the oocyte will be addressed. 


\section{MATERIALS AND METHODS}

\subsection{Materials}

\subsubsection{Model Organism Xenopus laevis}

In this study the African clawed frog Xenopus laevis (X. laevis) was used as model organism. The adult frogs were purchased from NASCO (Fort Atkinson, USA)

\subsubsection{Bacteria strains}

For cloning the Escherichia coli (E.coli) strain XL-1 was used: RecA1, endA1, gyrA96, thi-1, hsdR17, supE44, relA1, lac[F'proAB, laclqZ $\Delta M 15$, Tn10(Tetr)]c (Stratagene).

Protein expression studies were performed with BL21 (DE3) E.coli B F', ompT, hsdS ( $\mathrm{r}_{\mathrm{B}^{-}}$ $\left.\mathrm{m}_{\mathrm{B}^{-}}\right), \mathrm{dcm}^{+}$, $\mathrm{Tet}^{\mathrm{r}}$, gal $\lambda(\mathrm{DE} 3)$ end $A$ Hte [argU proLCamr] [argU ileY leuW Strep/Specr].

\subsubsection{Chemicals, Buffers, Media}

\subsubsection{Chemicals}

All chemicals were purchased from the following companies: Roth (Karlsruhe), SigmaAldrich Chemie (Taufkirchen), Applichem (Darmstadt), and Biochrom (Berlin), Life Technologies GmbH (Darmstadt), Roche (Mannheim), Thermo Fisher Scientific/Fermentas

- Germany GmbH (Schwerte).

\subsubsection{Buffers and Media}

All buffers were prepared using deionized water (MiliQ).

\section{Alkaline phosphatase buffer (APB)}

$100 \mathrm{mM}$ Tris, $50 \mathrm{mM} \mathrm{MgCl}_{2}, 100 \mathrm{mM} \mathrm{NaCl}, 0.1$ \% TWEEN-20; pH 9.0

\section{Bleaching solution}

$50 \%$ Formamide, $0,5 \% \mathrm{H}_{2} \mathrm{O}_{2}$, in $5 x \mathrm{SSC}$

\section{Blocking solution}

1x TBST; $5 \%$ nonfat dry milk 


\section{Buffer M}

$150 \mathrm{mM} \mathrm{NaCl}, 50 \mathrm{mM} \mathrm{NaH}_{2} \mathrm{PO}_{4}, \mathrm{pH}$ 7.8, 1 mM DTT, 15\% glycerol, 0.1\% Triton X-100

\section{Collagenase-buffer}

$82.5 \mathrm{mM} \mathrm{NaCl}, 2 \mathrm{mM} \mathrm{KCl}, 1 \mathrm{mM} \mathrm{MgCl}$, 5 mM HEPES; $\mathrm{pH} 7.5$

\section{Color reaction solution (WMISH)}

$80 \mu \mathrm{g} / \mathrm{ml} \mathrm{NBT}, 175 \mu \mathrm{g} / \mathrm{ml} \mathrm{BCIP}$ in APB; $\mathrm{pH} 9.0$

\section{Coomassie Destaining solution}

$40 \%$ ethanol, $10 \%$ acetic acid, $50 \% \mathrm{H}_{2} \mathrm{O}$

\section{Coomassie Staining solution}

$0.1 \%$ Coomassie blue, $10 \%$ acetic acid, $40 \%$ ethanol, $49.9 \% \mathrm{H}_{2} \mathrm{O}$

\section{Cystein solution}

$2 \%$ L-Cystein hydrochloride; $\mathrm{pH} 7.8$

\section{EMSA Loading Dye}

50 mM Tris- $\mathrm{Cl} \mathrm{pH} \mathrm{7.5,} 50$ \% glycerol, $0.01 \%$ Bromophenol blue

\section{HNTA buffer}

$1 \mathrm{M} \mathrm{NaCl}, 50 \mathrm{mM} \mathrm{Na}_{2} \mathrm{HPO}_{4}, \mathrm{pH}$ 7.8, 1 mM DTT, 1 \% Triton X-100

\section{HS-PBS}

73,1 g NaCl, $0.5 \mathrm{~g} \mathrm{KCl}, 0.8 \mathrm{~g} \mathrm{KH}_{2} \mathrm{PO}_{4}, 13.6 \mathrm{~g} \mathrm{Na}_{2} \mathrm{HPO}_{4}$, pH 7.3, 1 mM DTT, 0.1 \% Triton $\mathrm{X}-100$

\section{Hybridization Mix (Hyb Mix)}

$50 \%$ (v/v) Formamide (deionized), $1 \mathrm{mg} / \mathrm{ml}$ Torula-RNA (Sigma), 100 $\mathrm{gg} / \mathrm{ml}$ Heparin, $1 \mathrm{x}$ Denhardt's, $0.1 \%$ (v/v) Tween-20, $0.1 \%$ (v/v) CHAPS (Sigma), 10 mM EDTA, 5X SSC

\section{Injection buffer}

1x MBS, 1 \% Ficoll (Sigma) 
IPP145 buffer (10x) (Protein lysates, ColP embryos)

$100 \mathrm{mM}$ Tris (pH 8), $1450 \mathrm{mM} \mathrm{NaCl}, 1 \% \mathrm{NP} 40$ (v/v)

IPP145 buffer (1x)

$10 \%$ 10x IPP145 buffer (v/v), $5 \%$ Glycerol (v/v), Complete Protease inhibitor mix EDTA free (1/4 tablet per $50 \mathrm{ml}$ of buffer, Roche)

\section{LB-Agar}

$1.5 \%(\mathrm{w} / \mathrm{v})$ agar in liquid LB-medium

\section{Laemmli running buffer (1x)}

$25 \mathrm{mM}$ Tris, $192 \mathrm{mM}$ Glycine, $0.1 \%$ SDS

\section{Luria-Bertani (LB)-Medium}

$1 \%$ (w/v) Bacto-Trypton, $0.5 \%$ (w/v) yeast extract, $1 \%(\mathrm{w} / \mathrm{v}) \mathrm{NaCl}, \mathrm{pH} 7.5$

The medium was supplemented with Ampicillin $(50 \mu \mathrm{g} / \mathrm{ml})$, Kanamycin $(50 \mu \mathrm{g} / \mathrm{ml})$,

Tetracycline $(20 \mu \mathrm{g} / \mathrm{ml})$

\section{MAB (5x)}

$500 \mathrm{mM}$ maleic acid, $750 \mathrm{mM} \mathrm{NaCl}$; $\mathrm{pH} 7.5$

\section{MBS buffer (5x)}

$88 \mathrm{mM} \mathrm{NaCl}, 1 \mathrm{mM} \mathrm{KCl}, 0.82 \mathrm{mM} \mathrm{MgSO}_{4}, 10 \mathrm{mM}$ Hepes pH7.6, $2.4 \mathrm{mM} \mathrm{NaHCO}_{3}, 0.41$ $\mathrm{mM} \mathrm{CaCl}_{2}, 0.66 \mathrm{mM} \mathrm{KNO}_{3} ; \mathrm{pH} 7.4$

\section{MBS buffer (1x)}

$1 \mathrm{x}$ MBS buffer salts

\section{MEM (10x)}

1 M MOPS, 20 mM EGTA, 10 mM MgSO ${ }_{4}$ pH 7.4

\section{MEMFA (1x)}

$1 \times$ MEM, $4 \%$ formaldehyde 


\section{MNTA buffer}

$500 \mathrm{mM} \mathrm{NaCl}, 50 \mathrm{mM} \mathrm{Na}_{2} \mathrm{HPO}_{4}, \mathrm{pH}$ 7.8, 1 mM DTT, 1 \% Triton X-100

\section{Oocyte culture medium (OCM)}

50 \% Leibovitz L15 culture medium without L-glutamine (Invitrogen), 15 mM HEPES/NaOH (pH 7.5), 1 mM L-Glutamine (Invitrogen), $1 \mu \mathrm{g} / \mathrm{ml}$ Insulin (Sigma), 100 $\mu \mathrm{g} / \mathrm{ml}$ Gentamycin (Invitrogen), $50 \mu \mathrm{g} / \mathrm{ml}$ Tetracycline (Sigma), $50 \mathrm{U} / \mathrm{ml}$ Nystatin (Invitrogen)

\section{PBS (10x)}

$1.75 \mathrm{M} \mathrm{NaCl}, 1 \mathrm{M} \mathrm{KCl}, 65 \mathrm{mM} \mathrm{Na}_{2} \mathrm{HPO}_{4}, 18 \mathrm{mM} \mathrm{KH} \mathrm{PO}_{4} ; \mathrm{pH} 7.4$

\section{Ponceau S solution}

$2 \mathrm{~g}$ Ponceau S, $30 \mathrm{~g}$ trichloroacetic acid, $30 \mathrm{~g}$ sulfosalicylic acid per $100 \mathrm{ml}$

\section{Protein Blotting buffer (semi dry)}

39 mM Glycine, 48 mM Tris, $0.037 \%$ SDS (v/v), $20 \%$ methanol (v/v), pH 8.0

\section{PTw}

$0.1 \%$ Tween-20 in $1 \times$ PBS

\section{SDS Loading buffer (2x)}

$62,5 \mathrm{mM} 0.5 \mathrm{M}$ Tris (pH 6.8), 2 \% SDS (w/v), 10\% (v/v) glycerol, $700 \mathrm{mM} \beta$ mercaptoethanol, $0.05 \%(\mathrm{w} / \mathrm{v})$ bromphenol blue

SSC (20x)

$3 \mathrm{M} \mathrm{NaCl}, 0.3 \mathrm{M}$ Sodium citrate; pH 7.4

\section{TAE (Tris/Acetate/EDTA)}

40 mM Tris-Acetate (pH 8.5), 2 mM EDTA

\section{TBST (1x)}

50 mM Tris, 150 mM NaCl, 0.1 \% TWEEN-20; pH 7.5 


\section{5x UV- Crosslink buffer}

$5 \mathrm{mg} / \mathrm{ml}$ heparin, $1 \%$ glycerol, $50 \mathrm{mM} \mathrm{KCl,} 10 \mathrm{mM}$ DTT,

5,2 mM HEPES (pH 7.8), 1 mM MgCl2, $0.1 \mathrm{mM}$ EDTA, $40 \mu \mathrm{g} / \mathrm{ml}$ yeast tRNA

\section{YSS-ColP buffer (Cy3-RNA CoIP)}

$50 \mathrm{mM}$ Tris- $\mathrm{HCl}$ (pH 8), $75 \mathrm{mM} \mathrm{NaCl}, 1 \mathrm{mM} \mathrm{MgCl} 2,0.05 \%$ (v/v) NP-40, 10m mM

sucrose, $1 \mathrm{mM}$ DTT, Complete Protease inhibitor mix EDTA free (1/4 tablet per $50 \mathrm{ml}$ of buffer, Roche)

\subsubsection{Oligonucleotides}

Oligonucleotides were purchased desalted and lyophilized from Sigma-Aldrich Chemie. They were dissolved in the indicated volume of $\mathrm{HPLC} \mathrm{H}_{2} \mathrm{O}$ to obtain a primer stock concentration of $100 \mu \mathrm{M}$, which was stored at $-20^{\circ} \mathrm{C}$. For PCR an oligonucleotide concentration of $10 \mu \mathrm{M}$ was used.

\subsubsection{Sequencing oligonucleotides}

Table 2.1 Sequencing oligonucleotides and working conditions

\begin{tabular}{lll}
\hline Oligonucleotide & Sequence 5'--> $^{\prime} \mathbf{3}^{\prime}$ & $\left.\mathbf{T}_{\mathbf{A}} \mathbf{}^{\circ} \mathbf{C}\right]$ \\
\hline SP6 & TTAGGTGACACTATAGAATAC & 48 \\
T3 & AATTAACCCTCACTAAAGGG & 56 \\
T7 (pCS2+) & TCTACGTAATACGACTCACTATAG & 56 \\
T7 (pGEM-T) & TAATACGACTCACTATAGGGCGA & 56 \\
SP6 (pCMV-Sport6) & CTATTTAGGTGACACTATAG & 56 \\
T7 (pCMV-Sport6) & TAATACGACTCACGTATAGGG & 55 \\
M13 rev & AGCGGATAACAATTTCACAC & 56 \\
\hline
\end{tabular}




\subsubsection{Oligonucleotides for RT-PCR}

Table 2.2 RT-PCR oligonucleotides and working conditions

\begin{tabular}{lll}
\hline Oligonucleotide & Sequence 5'--> 3' & $\mathbf{T}_{\mathbf{A}}\left[{ }^{\circ} \mathbf{C}\right]$ \\
\hline ODC-F & GCCATTGTGAAGACTCTCTCCATTC & 56 \\
ODC-R & TTCGGGTGATTCCTTGCCAC & 56 \\
Dazl F RT & TATGTGCAACCTAGACCTGTGG & 58 \\
Dazl R RT & ATTGGTGGAGAGCTTGGATATG & 58 \\
GFP F2 jw & AGTGGAGAGGGTGAAGGTGA & 55 \\
GFP R2 & CTTCAGCACGTGTCTTGTAGT & 55 \\
Xpat_RT_F & AGCTCCAACTACGAGCCACA & 56 \\
Xpat_RT_R & ACATCGGACACAGCAAACCA & 56 \\
\hline
\end{tabular}

\subsubsection{Oligonucleotides for the amplification of XDE-LE subfragments}

Sequences of XDE-LE oligonucleotides are indicated in $5^{\prime} \rightarrow 3^{\prime}$ direction. The T7promotor region is underlined.

Table 2.3 XDE-LE oligonucleotides

\begin{tabular}{ll}
\hline Oligonucleotide & Sequence 5'--> 3' \\
\hline XDE-LE T7 5' F & TAATACGACTCACTATAGGGCTGCCCTTGCATCCTACA \\
XDE-LE T7 5'del1 F & TAATACGACTCACTATAGGGTTGCATTGGTGTAAAGCT \\
\hline XDE-LE T7 5'del2 F & TAATACGACTCACTATAGGGTCACTTGTTATTGCACTT \\
\hline XDE-LE T7 5'del3 F & TAATACGACTCACTATAGGGTGTATCTTGCACTTAAGA \\
\hline XDE-LE 3' R & AGGTGAAAATCAAGACAG \\
XDE-LE 3'del1 R & GGAAAGCCCTTTATGAAA \\
XDE-LE 3'del2 R & ATCGCCTCACTTCAAAGT \\
XDE-LE 3'del3 R & GCAGGGAGCAGGCAGATG \\
XDE-LE 3'del4 R & TTTTCCGATCTTAAGTGC \\
XDE-LE 3'del5 R & AAAGTGCAATAACAAGTG \\
XDE-LE 3'del6 & AATAAAGTGAAACAAAAATTAGCTTTACACC \\
XDE-LE 3'del7 & AGCTTTACACCAATGCAAAAA \\
\hline
\end{tabular}

All oligonucleotides except XDE-LE 3'del6 and XDE-LE 3'del7 were designed by Diana Bauermeister. 


\subsubsection{Oligonucleotides for the amplification of XDazl-LE subfragments}

XDazI-LE oligonucleotides are indicated in $5^{\prime} \rightarrow 3^{\prime}$ direction. The T7-promotor region is underlined.

Table 2.4 XDazl-LE oligonucleotides

\begin{tabular}{ll}
\hline Oligonucleotide & Sequence 5'--> 3' \\
\cline { 2 - 2 } T7-XDazl-up1 & TAATACGACTCACTATAGGGATCACTGAGGGGCTTGAG \\
T7-XDazl-up2 & TAATACGACTCACTATAGGGATTTTGTCATTTCCATTT \\
T7-XDazl-up3 & TAATACGACTCACTATAGGGTGTCTGTACACACTTCTA \\
T7-XDazl-up4 & TAATACGACTCACTATAGGGGGTCACTGTTTCACATTG \\
T7-XDazl-up5 & TAATACGACTCACTATAGGGTGTGCACATACACAATGA \\
T7-XDazl-up5.1 & TAATACGACTCACTATAGGGTGATGAGCCGCTTCCCCT \\
T7-XDazl-up6 & TAATACGACTCACTATAGGGATTGTGTAGCTTGAGGGT \\
XDazl-low1 & ACGGGGCTATGTGGCTAA \\
XDazl-low2 & GTGTTTATTTTAACCCTC \\
XDazl-low3 & CATGGGATTAAGTCATTG \\
XDazl-low4 & TTGGGCAGTGAAGTATAA \\
XDazl-low5 & CAAACAGAAAAGGTTGTG \\
XDazl-LE low5.1 & CATAAAACATGTGACTCCTG \\
XDazl-low6 & AACAATCTTATTCTGAAT \\
\hline
\end{tabular}

2.1.4.5 Oligonucleotides for the amplification of XDazI-LE reporter subfragments

Table 2.5 XDazl-LE oligonucleotides for GFP-reporter cloning

\begin{tabular}{ll}
\hline \multicolumn{1}{c}{ Oligonucleotide } & \multicolumn{1}{c}{ Sequence 5'--> 3' } \\
\hline XDAZL-LE up2 +Xhol & CGCTCGAGATTTTGTCATTTCCATTT \\
XDAZL-LE up3 +Xhol & CGETCGAGTGTCTGTACACACTTCTA \\
XDAZL-LE low2 +Notl & CGGCGGCCGCGTGTTTATTTTAACCCTC \\
XDAZL-LE low3 +Notl & CGGCGGCCGCCATGGGATTAAGTCATTG \\
XDazl-up5.1 +Xhol & CGCTCGAGTGATGAGCCGCTTCCCCT \\
XDazl-LE low5.1+ Notl & GCGCGGCCGCCATAAAACATGTGACTC \\
\hline
\end{tabular}


2.1.4.6 Oligonucleotides for the $X$. laevis/X. tropicalis hybrid analysis

Table 2.6 RT-PCR oligonucleotides and working conditions

\begin{tabular}{lll}
\hline Oligo name & Sequence 5'--> 3' & $\mathbf{T}_{\mathbf{A}}\left[{ }^{\circ} \mathbf{C}\right]$ \\
\hline Dazl X.t.F & GCCCTGCCATTCGAAAGATGC & 56 \\
Dazl X.t.R_2 & ATTAGTGTTAGGTGAATTCGACAAACT & 56 \\
Dazl X.I._X.t.F & CCTGCTTGTTTGATCCAGC & 56 \\
DazI X.I._R & CACAATATAACATCAAAGGAGAAAGT & 56 \\
DeadSouth X.I._X.t. F & GTGTACATGCTAAAGGAGATTGAAG & 56 \\
DeadSouth X.t. R & CATAAGAACATTAGTGGGAAAACA & 56 \\
DeadSouth X.I. R & GCTGTGAAGTGGGCGATTCTA & 56 \\
DE X.I._X.t.F & CGATTTTGGTACCAAGTAGTCA & 56 \\
DE X.t._R & AACCCTTACACCACAAGTTTTG & 56 \\
DazI X.I.R_2 & CTTATTCTGAATACTAAAATGGAAATGAC & 56 \\
\hline
\end{tabular}

2.1.4.7 Oligonucleotides used for the generation of XDazl ORF deletion mutants

Table 2.7 Oligonucleotides used for the cloning of the XDazl ORF deletion mutants

\begin{tabular}{cl}
\hline \multicolumn{1}{c}{ Name } & \multicolumn{1}{c}{ Sequence 5'--> 3' } \\
\hline DAZL del RNP1 $F$ & [Phos]-ATTTCTTTTTCTGATGAGGTGGATGT \\
DAZL del RNP1 R & [Phos]-GGACACTCCTGTCCGGTCAGT \\
DAZL del RNP2 F & [Phos]-GATATTACGATGGATGAAATAGAAATTCG \\
DAZL del RNP2 R & [Phos]-TGTGTTTGGCATGATTTTACCCTCT \\
\hline
\end{tabular}




\subsubsection{Morpholino oligonucleotides}

Antisense Morpholino oligonucleotides (Morpholino, MO) were purchased from Gene Tools, LLC (Philomath, USA). Morpholinos were dissolved in RNase-free water to a $1 \mu \mathrm{M}$ concentration and stored as aliquots at $-20^{\circ} \mathrm{C}$.

Table 2.8 Antisense Morpholino oligonucleotides

\begin{tabular}{ll}
\hline Morpholino name & Sequence 5'-->3' $^{\prime}$ \\
\hline XDead end MO & CTGCTCGTCTGACAGCTTCATTTTC \\
XDazl MO & AAACCACACAACCCGAACAAACCAT \\
DazI-LE MO1 & TTGTGTACTTAGAAGTGTGTACAGA \\
DazI-LE MO2 & TAAAGGTGCATACAAACAGAAAAGG \\
DazI-LE MO3 & AGTGACCAAACTGTAGTTGTCTACT \\
DazI-LE MO4 & GAAGTATAAATTTCAATGTGAAACA \\
DazI-LE MO5 & AAAAGATCCAGTGCTCTTGGCAGTG \\
DazI-LE MO6 & TATGTGCACACCACTTGTGAGAGAA \\
DazI-LE MO7 & AGTGACATGGGATTAAGTCATTGTG \\
Control MO & CCTCTTACCTCAGTTACAATTTATA \\
\hline
\end{tabular}

\subsubsection{Kits for nucleic acid analysis}

Table 2.9 Commercially available Kits and method

\begin{tabular}{|l|l|l|}
\hline Method & \multicolumn{2}{l}{ Kit } \\
\hline DNA Isolation & $\begin{array}{l}\text { Invisorb DNA } \\
\text { CleanUp, Mini Kit }\end{array}$ & Stratec biomedical, Birkenfeld \\
\hline $\begin{array}{l}\text { RNA Cleanup, } \\
\text { Fluorescence-, Dig- } \\
\text { probes }\end{array}$ & RNeasy Mini Kit & Qiagen, Germany \\
\hline Cap mRNA CleanUp & $\begin{array}{l}\text { illustra RNA spin } \\
\text { Mini Kit }\end{array}$ & GE Healthcare, Freiburg \\
\hline Gel Extraction & $\begin{array}{l}\text { Invisorb DNA } \\
\text { CleanUp, Mini Kit }\end{array}$ & Stratec biomedical, Birkenfeld \\
\hline Plasmid Isolation Midi & $\begin{array}{l}\text { NucleoBond Xtra } \\
\text { Midi/ Maxi }\end{array}$ & Macherey-Nagel, Düren \\
\hline Plasmid Isolation Mini & $\begin{array}{l}\text { GeneJET Plasmid } \\
\text { Miniprep Kit }\end{array}$ & Thermo Scientific, Schwerte \\
\hline
\end{tabular}




\subsubsection{Constructs}

\subsubsection{Plasmids}

\section{pCS2+}

The pCS2+ vector is applicable for expression experiments in Xenopus. It contains the simian cytomegalovirus IE94 enhancer/promoter sequence, a viral SP6 promoter, polylinker sequence and SV40 viral polyadenylation signal allowing the in vitro transcription of polyadenylated sense mRNA for microinjection. A T7 promoter in reverse orientation between the polylinker and the SV40 polyA site allows antisense probe synthesis (Rupp et al. 1994).

\section{FLAG-pCS2+}

FLAG-pCS2+ contains the pCS2+ vector backbone and the peptide sequence of the FLAG-tag: DYKDDDDK at the 5'-end of the first polylinker (Rupp et al. 1994). This vector allows the expression of FLAG-epitope tagged proteins.

\section{MT-pCS2+}

MT-pCS2+ contains the pCS2+ vector backbone and the peptide sequence of the hexameric repeat of the Myc epitope tag at the 5'-end of the first polylinker (Rupp et al. 1994). This vector allows the expression of Myc epitope tagged proteins.

\section{pGEM-T easy}

The pGEM-T easy vector is a commercially available system for the cloning of PCR products. It contains single $3^{\prime}-T$ overhangs at the insertion site within the multiple cloning site and a T7 or SP6 RNA polymerase promoter. This cloning site is located within the alpha-peptide coding region of the enzyme beta-galactosidase and allows blue/white selection on IPTG/Xgal plates (Promega).

\section{pBluescript KS/SK}

The pBluescript KS/SK is a commercially available phagemid (plasmids with a phage origin) that includes the beta-galactosidase coding region, which is interrupted by a polylinker and flanked by T7 and T3 RNA polymerase promoters (Stratagene). 


\section{pSP64-mGFP}

The pSP64-mGFP5 expression vector was obtained from E. Raz, Institute of Cell Biology, Münster, Germany and was used for the expression of reporter constructs by including the sequences of the Xenopus 3' UTRs of interest.

\subsubsection{Constructs for recombinant protein expression}

\section{pGEX6p3 Dazl RRM}

The pGEX6p3 is a bacterial vector with a tac promoter for chemically inducible, highlevel expression of GST-tagged recombinant proteins (GE Healthcare). Very mild elution conditions can be used for the release of fusion proteins from the affinity matrix, thus minimizing effects on antigenicity and functional activity. The vector contains a PreScission Protease recognition sites for cleaving the desired protein from the fusion product. The vector mediates the resistance against the antibiotic ampicillin. The XDazl RRM was cloned into the vector using the DAZL RRM F2 (CGGAATTCGGTGTTGCCAGAGG) oligonucleotide and the DAZL RRM R (CGCTCGAGTCAACAAATTTTTC) oligonucleotide.

\section{PETM30 XDE ORF}

The $\mathrm{pETM}$ vectors are derived from $\mathrm{pET}$ (Novagene) backbones. They share some common features like the 6xHis tag, a TEV protease recognition site and the conserved multiple cloning site (MCS) starting with an Ncol recognition site. An important characteristic of the pETM30 vector is the introduced GST tag beside the His tag, allowing the expression of proteins with both tags. The vector mediates the resistance against the antibiotic kanamycin. The XDE ORF was cloned with $\mathrm{Ncol}$ and Xhol restriction sites into the vector and was available in the laboratory, cloned by Ines Eckardt (Marker box: III-5J).

\subsubsection{Constructs used for antisense probe transcription}

Xpat: (Hudson and Woodland 1998b) $2 \mathrm{~kb}$ of the Xpat 3'-UTR were cloned into the pBluescript SK-vector. For preparation of the antisense RNA, the construct was linearized using the $E c o R /$ restriction site and transcribed with the T7-Polymerase. 
mGFP5: (Koebernick 2010) 717 bp of the GFP ORF were cloned into the pGEM-T easy vector. For preparation of the antisense RNA, the construct was linearized using the Spel restriction site and transcribed with the T7-Polymerase.

\subsubsection{Overexpression constructs and reporters}

Table 2.10 Expression and reporter constructs - available in the laboratory

\begin{tabular}{l|c|c|c}
\hline \multirow{2}{*}{ Construct name } & \multicolumn{2}{c|}{ sense RNA } & \multirow{2}{*}{ Source } \\
\cline { 2 - 3 } & cut & polymerase & \\
\hline pSP64-GFP-XDazl LE & Notl & Sp6 & (Koebernick 2010) \\
\hline pSP64-GFP-Xcat2 LE & Notl & Sp6 & (Koebernick 2010) \\
\hline pSP64-GFP-Xpat LE & Notl & Sp6 & (Koebernick 2010) \\
\hline $\begin{array}{l}\text { pSP64mGFP-DE3'UTR-F5 } \\
\text { (XDE-LE-R) }\end{array}$ & Notl & Sp6 & (Koebernick 2010) \\
\hline pCS2+-flag-Dazl & Notl & Sp6 & JL 75 \\
\hline ElrB1 in pCS2+Flag & Xbal & Sp6 & MC 303 \\
\hline pCS2+Flag-XDE 1d.4 & Notl & Sp6 & III-4F \\
\hline
\end{tabular}

XDazl-LE reporter constructs were cloned using the depicted oligonucleotides in Table 2.5

Table 2.11 XDazl-LE reporter constructs and in vitro transcription conditions

\begin{tabular}{l|l|c|c}
\hline \multicolumn{1}{c|}{ Construct name } & \multicolumn{2}{|c|}{ Oligonucleotide pair } & \multicolumn{2}{c}{ sense RNA } \\
\cline { 3 - 4 } & & cut & polymerase \\
\hline pSP64-mGFP-XDazl-LE I & XDAZL-LE up2 +Xhol/XDAZL-LE low2 +Notl & Notl & Sp6 \\
\hline pSP64-mGFP-XDazl-LE m1 & XDAZL-LE up2 +Xhol/XDAZL-LE low3 +Notl & Notl & Sp6 \\
\hline pSP64-mGFP-XDazl-LE m2 & XDAZL-LE up3 +Xhol/XDAZL-LE low2 +Notl & Notl & Sp6 \\
\hline pSP64-mGFP-XDazl-LE s2 & XDAZL-LE up3 +Xhol/XDAZL-LE low3 +Notl & Notl & Sp6 \\
\hline
\end{tabular}

\section{pCS2-Flag XDazl del RNP1, pCS2-Flag XDazl del RNP2, pCS2-Flag XDazl del RNP1+2}

These constructs harbor the XDazl ORF with the deletion of either RNP1, RNP2 or both RNPs in the pCS2-Flag vector. For mRNA preparation the constructs were linearized using the Not/ restriction site and transcribed with the Sp6-Polymerase. Deletion mutants were generated by using the oligonucleotides in Table 2.7. 


\subsubsection{Constructs used for the generation of Cy3-labeled RNA}

Table 2.12 Constructs used for the generation of Cy3-labeled RNA and in vitro transcription conditions

\begin{tabular}{l|l|l|l}
\hline \multicolumn{1}{c|}{ Construct name } & \multicolumn{2}{c|}{ sense RNA } & \multirow{2}{*}{ Source } \\
\cline { 2 - 4 } & cut & polymerase & \\
\hline pGEM-T-XDazl LE & Ndel & $T 7$ & JL 85 \\
\hline pGEM-T-Xcat2 LE & Ndel & $T 7$ & JL 86 \\
\hline pGEM-T-Xpat LE & Ndel & $T 7$ & JL 87 \\
\hline XDE-LE F2 in pGEM-T & Xhol & $T 7$ & MC 319 \\
\hline B-Globin 3 UTR in pGEM-Teasy & Xhol & T7 & MC 364 \\
\hline
\end{tabular}

\subsubsection{PCR fragments used for the generation of Cy3-labeled RNA}

Table 2.13 PCR-fragment name and oligonucleotide pair used for the amplification of PCR products applied to the in vitro transcription of XDE-LE Cy3-labeled RNA

\begin{tabular}{ll}
\hline PCR fragment name & Oligonucleotide pair \\
\hline XDE-LE FL & XDE-LE T7 5' F+XDE-LE 3' R \\
XDE-LE 5'del1 & XDE-LE T7 5'del1 F+XDE-LE 3' R \\
\hline XDE-LE 5'del2 & XDE-LE T7 5'del2 F+XDE-LE 3' R \\
XDE-LE 5'del3 & XDE-LE T7 5'del3 F+XDE-LE 3' R \\
\hline XDE-LE 3'del1 & XDE-LE T7 5' F+XDE-LE 3'del1 R \\
XDE-LE 3'del2 & XDE-LE T7 5' F+XDE-LE 3'del2 R \\
XDE-LE 3'del3 & XDE-LE T7 5' F+XDE-LE 3'del3 R \\
XDE-LE 3'del4 & XDE-LE T7 5' F+XDE-LE 3'del4 R \\
\hline XDE-LE 3'del5 & XDE-LE T7 5' F+XDE-LE 3'del5 R \\
XDE-LE 3'del6 & XDE-LE T7 5' F+XDE-LE 3'del6 \\
XDE-LE 3'del7 & XDE-LE T7 5' F+XDE-LE 3'del7 \\
\hline
\end{tabular}


Table 2.14 PCR-fragment name and oligonucleotide pair used for the amplification of PCR products applied to the in vitro transcription of XDazl-LE Cy3-labeled RNA

\begin{tabular}{ll}
\hline PCR-fragment name & Oligonucleotide pair \\
\hline XDazl-LE FL & T7-XDazl-up1+XDazl-low1 \\
\hline XDazl-LE 5'del1 & T7-XDazl-up2+XDazl-low1 \\
\hline XDazl-LE 5'del2 & T7-XDazl-up3+XDazl-low1 \\
XDazl-LE 5'del3 & T7-XDazl-up4+XDazl-low1 \\
\hline XDazl-LE 5'del4 & T7-XDazl-up5+XDazl-low1 \\
\hline XDazl-LE 5'del5 & T7-XDazl-up6+XDazl-low1 \\
\hline XDazl-LE 3'del1 & T7-XDazl-up1+XDazl-low2 \\
XDazl-LE 3'del2 & T7-XDazl-up1+XDazl-low3 \\
\hline XDazl-LE 3'del3 & T7-XDazl-up1+XDazl-low4 \\
XDazl-LE 3'del4 & T7-XDazl-up1+XDazl-low5 \\
\hline XDazl-LE 3'del5 & T7-XDazl-up1+XDazl-low6 \\
XDazl-LE I & T7-XDazl-up2+XDazl-low2 \\
\hline XDazl-LE m1 & T7-XDazl-up2+XDazl-low3 \\
XDazl-LE m2 & T7-XDazl-up3+XDazl-low2 \\
XDazl-LE s1 & T7-XDazl-up1+XDazl-low5.1 \\
XDazl-LE s2 & T7-XDazl-up3+XDazl-low3 \\
\hline XDazl-LE s3 & T7-XDazl-up5.1+XDazl-low1 \\
\hline
\end{tabular}

\subsubsection{Antibodies}

Table 2.15 Primary antibodies

\begin{tabular}{cccc}
\hline Name & Company & Source & Dilution \\
\hline Flag & Sigma & Rabbit & $1: 500$ \\
HuR & Santa Cruz & Mouse & $1: 2000$ \\
XDazl & Mita, 2000 & Mouse & $1: 10000$ \\
XStaufen & Allison, 2004 & Rabbit & $1: 10000$ \\
\hline
\end{tabular}

Table 2.16 Secondary antibodies

\begin{tabular}{cccc}
\hline Name & Company & Source & Dilution \\
\hline anti-Mouse-HRP & Santa Cruz & Goat & $1: 5000$ \\
anti-Rabbit HRP & Cell Signaling & Goat & $1: 3000$ \\
IRDye $\AA$ 800CW Goat anti-Mouse & LI-COR & Goat & $1: 20000$ \\
IRDye ${ }^{\circledR} 800 \mathrm{CW}$ Goat anti-Rabbit & LI-COR & Goat & $1: 20000$ \\
IRDye ${ }^{\circledR} 680 \mathrm{CW}$ Goat anti-Mouse & LI-COR & Goat & $1: 20000$ \\
IRDye $\AA$ 680CW Goat anti-Rabbit & LI-COR & Goat & $1: 20000$ \\
\hline
\end{tabular}




\subsubsection{SiRNA}

The siRNA was purchased from Eurofins MWG Operon, Ebersberg. The siRNA was dissolved in 1x siMAX Universial Buffer (5x stock: 30 mM HEPES, $100 \mathrm{mM} \mathrm{KCl}, 1 \mathrm{mM}$ $\mathrm{MgCl}_{2} ; \mathrm{pH} 7.3$ ) and stored in $25 \mathrm{mM}$ aliquots at $-20^{\circ} \mathrm{C}$.

(guide strand in bold)

siRNA-NS

5'-UAGCGACUAAACACAUCAUdTdT-3' 5'-AUGAUGUGUUUAGUCGCUAdTdT-3'

\subsubsection{Equipment List}

All commodities and appliances were purchased from the companies as follows:

Eppendorf (Hamburg), Falcon (Heidelberg), Schütt (Göttingen), Greiner (Frickenhausen), Qiagen (Hilden), Sarstedt (Nürnbrecht), Thermo Fisher Scientific (Karlsruhe), Perkin Elmer (Rodgau), Boehringer Ingelheim (Ingelheim am Rhein), life Technologies (Darmstadt).

NanoDrop-2000c Spectrophotometer (Thermo Fisher Scientific)

UV-transilluminator (Bio-Rad)

GDS documentation system (INTAS)

Thermomixer comfort (Eppendorf)

ABI 3130 xI Genetic Analyzer (Applied Biosystems).

SteREO Lumar.V12 (Zeiss)

LSM780 (Zeiss)

Typhoon 9400 (GE Healthcare Life Sciences)

Fastblot B34/B44 (Biometra)

Needle puller PN-30 (Narishige) 


\subsection{Methods}

All molecular biology standard techniques, including polymerase chain reaction, analysis and enzymatic manipulation of DNA and RNA, protein synthesis and plasmid propagation were performed according to Sambrook (Sambrook 2001).

\subsubsection{Cloning of PCR fragments}

For cloning of PCR fragments the High Fidelity PCR enzyme Mix (Fermentas) was used to amplify the fragments. PCR fragments were loaded onto a $1.5 \%$ agarose gel, excised from the gel or directly extracted from the PCR mixture with the Invisorb DNA CleanUp Mini Kit and eluted in $15 \mu \mathrm{l}$ Nase free $\mathrm{H}_{2} \mathrm{O}$.

\begin{tabular}{lc}
\hline Ingredients & Final conc. \\
\hline Plasmid DNA & $0.1 \mathrm{ng} / \mu \mathrm{l}$ \\
Primer 1 & $0.75 \mu \mathrm{M}$ \\
Primer 2 & $0.75 \mu \mathrm{M}$ \\
10x PCR buffer & $1 \mathrm{x}$ \\
dNTP mix & $0.2 \mathrm{mM}$ \\
High Fidelity Pol & $0.1 \mathrm{U} / \mu \mathrm{l}$ \\
Add H2O & \\
\hline Volume $_{\text {total }}$ & $50 \mu \mathrm{l}$ \\
\hline
\end{tabular}

\begin{tabular}{ccc}
\hline Temp [ ${ }^{\circ} \mathbf{C}$ ] & Duration [s] & Cycles \\
\hline 95 & 300 & 1 \\
\hline 95 & 45 & \\
$x$ & 45 & 30 \\
72 & $60 / 1 \mathrm{~kb}$ & \\
\hline 72 & 300 & 1 \\
\hline 8 & $\infty$ & 1 \\
\hline
\end{tabular}

Ligation

For ligation of DNA fragments 5 U T4 DNA ligase (Fermentas, Germany) were used for a single reaction in a total volume of $10 \mu \mathrm{l}$. To calculate the appropriate amount of PCR product (insert) applied to the ligation reaction, the following equation was used:

$\frac{\mathrm{ng} \text { of vector } \times \mathrm{kb} \text { size of insert }}{\mathrm{kb} \text { size of vector }} \times \frac{\text { insert }}{\text { vector molar ratio }}=\mathrm{ng}$ of insert

The ligation reaction was incubated overnight at $4{ }^{\circ} \mathrm{C}$. The following day, transformation into bacterial cells was performed. The ligation reaction for the generation of deletion mutants (XDazl del RNP1/2) was incubated for $30 \mathrm{~min}$ at RT before the transformation into bacterial cells was performed. 


\section{Chemical transformation and cultivation of bacterial cells}

Competent E.coli XL1 cells were used for the transformation. Cells were thawed on ice and to $200 \mu \mathrm{l}$ of cells $5 \mu \mathrm{l}$ of incubated ligation reaction mix were added. After gently flicking the tubes and incubation on ice for 30 minutes the cells were transformed via a heat-shock for 90 seconds at $42{ }^{\circ} \mathrm{C}$. Immediately the tubes were returned to ice for 3 minutes and $800 \mu \mathrm{l}$ room-temperature LB-medium was added. The reaction mix was incubated for $45-90 \mathrm{~min}$ at $37^{\circ} \mathrm{C}$, shook ( 150 rpm), centrifuged and subsequently 100 $\mu l$ fresh LB medium were added to the bacteria pellet. The bacterial cultures were plated on LB plates (containing the appropriate antibiotic and/or IPTG/XGal; IPTG (100 mM stock, $100 \mu \mathrm{l} /$ plate), X-gal (100 mM stock, $100 \mu / /$ plate)) and incubated overnight at $37^{\circ} \mathrm{C}$ (Sambrook 2001). The next day, the plates were checked for colony formation. In case of IPTG/X-gal supplement, white colonies generally contain inserts; blue colonies should not contain inserts. Single colonies were picked, transferred into LB-medium containing $100 \mu \mathrm{g} / \mathrm{ml}$ ampicillin (or corresponding antibiotic) and incubated overnight on a rotary shaker (220rpm) at $37{ }^{\circ} \mathrm{C}$. The bacteria were pelleted and stored at $-20{ }^{\circ} \mathrm{C}$ or directly used for plasmid isolation.

\subsubsection{RNA techniques}

\subsubsection{In vitro synthesis of capped sense mRNA}

In vitro capped sense mRNA was synthesized using the "SP6/T3/T7 mMESSAGE mMACHINE" Kit (Ambion Inc.). For a $10 \mu \mathrm{l}$ reaction, $3 \mu \mathrm{l}$ of linearized plasmid was added. The purification of the synthesized mRNA was performed using the "Illustra RNASpin Mini" Kit. The mRNA was eluted in $15 \mu \mathrm{l}$ RNase-free water at room temperature and the RNA concentration was measured using the NanoDrop 2000c spectrophotometer. To analyze the quality of the mRNA $0.5 \mu \mathrm{l}$ were mixed with Gel Loading buffer II and analyzed on a $1 \%$ agarose gel. For long time storage the RNA was aliquoted and stored at $-80^{\circ} \mathrm{C}$.

\subsubsection{In vitro synthesis of Cy3-labeled and antisense RNA}

Cy3-labeled RNAs were used for Electrophoretic Mobility Shift Assay (EMSA) analysis and Co-Immunoprecipitation (CoIP) experiments with in vitro translated proteins. RNA antisense probes were used in the whole mount in situ hybridization to visualize in vivo transcripts. To synthesize antisense RNA, $1 \mu \mathrm{g}$ of the linearized plasmid or amplified PCR product containing a T7 promoter sequence was used in a $25 \mu$ l labeling reaction. 
PCR reaction for the amplification of T7-XDE-LE/XDazI-LE fragments:

\begin{tabular}{lc}
\hline Ingredients & Final conc. \\
\hline Plasmid DNA & $0.2 \mathrm{ng} / \mu \mathrm{l}$ \\
Primer $1(10 \mu \mathrm{M})$ & $1 \mu \mathrm{l}$ \\
Primer $2(10 \mu \mathrm{M})$ & $1 \mu \mathrm{l}$ \\
10x Dream Taq Pu & $5 \mu \mathrm{l}$ \\
dNTP mix $(10 \mathrm{mM})$ & $1 \mu \mathrm{l}$ \\
$\mathrm{MgCl}_{2}(25 \mathrm{mM})$ & $0.6 \mu \mathrm{l}$ \\
Dream Taq Pol & $0.1 \mu \mathrm{l}$ \\
Add H2O & $50 \mu \mathrm{l}$ \\
\hline
\end{tabular}

\begin{tabular}{ccc}
\hline Temp $\left[{ }^{\circ} \mathbf{C}\right]$ & $\begin{array}{c}\text { Duration } \\
{[\mathbf{s}]}\end{array}$ & Cycles \\
\hline 95 & 120 & 1 \\
\hline 95 & 50 & \\
48 & 50 & 40 \\
72 & 50 & \\
\hline 72 & 180 & 1 \\
\hline 8 & $\infty$ & 1 \\
\hline
\end{tabular}

The PCR fragments were purified using the Invisorb DNA CleanUp Mini Kit.

A standard reaction for Dig/Cy3-labeling contained:

\begin{tabular}{lc}
\hline \multicolumn{1}{c}{ Ingredients } & Volume \\
\hline linearized template $(0.2 \mu \mathrm{g} / \mu \mathrm{ll})$ & $5 \mu \mathrm{l}$ \\
$5 x$ transcription buffer (Fermentas) & $5 \mu \mathrm{l}$ \\
rATP $(10 \mathrm{mM})$ & $1 \mu \mathrm{l}$ \\
rGTP $(10 \mathrm{mM})$ & $1 \mu \mathrm{l}$ \\
rCTP $(10 \mathrm{mM})$ & $1 \mu \mathrm{l}$ \\
rUTP $(10 \mathrm{mM})$ & $0.64 \mu \mathrm{l}$ \\
Dig - rUTP / Cy3 - rUTP (Boehringer/ PerkinElmer) & $0.36 \mu \mathrm{l}$ \\
DTT $(750 \mathrm{mM})$ & $1 \mu \mathrm{l}$ \\
Ribolock RNase inhibitor $(40 \mathrm{U} / \mu \mathrm{l}$, Fermentas $)$ & $1 \mu \mathrm{l}$ \\
Sp6/T7/T3 RNA polymerase $(20 \mathrm{U} / \mu \mathrm{l})$ & $1 \mu \mathrm{l}$ \\
Pyro-Phosphatase & $1 \mu \mathrm{l}$ \\
RNase free $\mathrm{H}_{2} \mathrm{O}$ & $\mathrm{add}$ to $25 \mu \mathrm{l}$ \\
\hline
\end{tabular}

The reaction was incubated for 2 hours at $37^{\circ} \mathrm{C}$ for the Dig-labeling and $4-12 \mathrm{~h}$ for Cy3labeling. After the labeling reaction, the DNA template was digested by the addition of TURBO DNase (2 $\mathrm{U} / \mu \mathrm{l}$, Ambion) for $15 \mathrm{~min}$ at $37^{\circ} \mathrm{C}$. The RNA was purified using the RNeasy Mini Kit (Qiagen). RNA elution was performed using 40-50 $\mu$ RNase-free water at $80{ }^{\circ} \mathrm{C}$. The quality of the antisense RNA was analyzed on a $1 \%$ agarose gel, according to the RNA quantity different Hyb mix volumes were immediately added to the antisense RNA and stored at $-20^{\circ} \mathrm{C}$. Cy3-labeled RNA was analyzed on an EtBr-free 1 $\%$ agarose gel. 


\subsubsection{Isolation of total RNA with trizol (TriFast)}

Snap frozen embryos or oocytes were lyzed in $500 \mu$ peqGOLD TriFast (peQlab) with a Omnican 40 syringe (Braun) and vortexed for $30 \mathrm{sec}, 80 \mu \mathrm{l}$ chloroform was added, sample was vortexed and centrifuged for $10 \mathrm{~min}$ at $4{ }^{\circ} \mathrm{C}$ and $13000 \mathrm{rpm}$. After centrifugation, the upper phase, containing the total RNA (approximately $200 \mu \mathrm{l}$ ), was transferred to a new eppendorf tube. $200 \mu \mathrm{l}$ chloroform was added and the sample was vortexed again for $30 \mathrm{sec}$ and centrifuged for $10 \mathrm{~min}$ at $4{ }^{\circ} \mathrm{C}$. The upper phase was recovered and transferred into a new eppendorf tube and $500 \mu \mathrm{l}$ isopropanol was added, mixed well and incubated at $-20{ }^{\circ} \mathrm{C}$ overnight. After $30 \mathrm{~min}$ of centrifugation at $4{ }^{\circ} \mathrm{C}$ and $13000 \mathrm{rpm}$, the pellet was washed with $500 \mu \mathrm{l}$ of $70 \%$ ethanol and centrifuged for $5 \mathrm{~min}$ at $4{ }^{\circ} \mathrm{C}$. The pellet was air-dried and resolved in $12.5 \mu \mathrm{l}$ RNase-free water. Genomic DNA

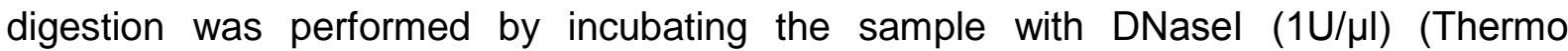
Scientific) at $37^{\circ} \mathrm{C}$ for $1.5 \mathrm{~h}$. The DNasel activity was inhibited by incubation for $10 \mathrm{~min}$ at $70^{\circ} \mathrm{C}$. RNA concentrations were measured using the NanoDrop.

\subsubsection{Reverse transcription (cDNA synthesis)}

cDNA was synthesized in a $10 \mu \mathrm{l}$ reaction from $100 \mathrm{ng}$ total RNA by MuLV Reverse Transcriptase (Roche).

\begin{tabular}{ll}
$1 \mathrm{x}$ & Go Taq Flex \\
$5 \mathrm{mM}$ & $\mathrm{MgCl}_{2}$ (Fer \\
$5 \mathrm{mM}$ & dNTP mix \\
$2.5 \mathrm{ng}$ & Random h \\
$100 \mathrm{ng}$ & Total RNA \\
$0.8 \mathrm{U} / \mu \mathrm{l}$ & Ribolock R \\
$20 \mathrm{U} / \mu \mathrm{l}$ & MuLV Rev \\
& \\
& \\
\hline Temp $\left[{ }^{\circ} \mathrm{C}\right]$ & Duration [min] \\
\hline 20 & 20 \\
42 & 60 \\
95 & 10
\end{tabular}




\subsubsection{Semiquantitaive real-time PCR}

For semiquantitative RT-PCR analysis a standard PCR reaction was carried out (Mullis 1986). $2 \mu \mathrm{l}$ cDNA was added to a $12.5 \mu$ total reaction volume. The standard $1 \mathrm{x}$ reaction mix contained: 1x Green Go Taq Flexi Buffer (Promega), $0.2 \mu \mathrm{M}$ RT oligonucleotides each, $1.5 \mathrm{mM} \mathrm{MgCl}_{2}$ and $0.5 \mathrm{U}$ Go Taq polymerase. To test for DNA contaminations ODC RT-PCR was carried out using total RNA. ODC RT-PCR on cDNA templates served as a control for equal cDNA concentrations.

\begin{tabular}{ccc}
\hline Temp [ ${ }^{\circ} \mathbf{C}$ ] & Duration [s] & Cycles \\
\hline 94 & 120 & 1 \\
\hline 94 & 45 & \\
$48-65$ & 45 & $26-35$ \\
72 & 45 & \\
\hline 72 & 420 & 1 \\
\hline 8 & $\infty$ & 1 \\
\hline
\end{tabular}

The cycle numbers and annealing temperatures were adapted to the oligonucleotides. The RT-PCR products were analyzed on a $2 \%$ agarose gel.

\subsubsection{Quantitative real-time PCR}

Quantitative real-time PCR analysis was performed using $2.5 \mu \mathrm{l}$ cDNA in a $20 \mu \mathrm{l}$ reaction volume in 96-well plates. The 1x standard reaction mix contained: 1x iQ SYBRGreen Supermix (Biorad) and $0.2 \mu \mathrm{M}$ RT-oligonucleotides each. The real-time RT-PCR was performed with the CFX96 Real-Time PCR Detection System. All measurements were performed in duplicates and normalized to the values of ornithine decarboxylase (ODC). Two independent biological replicates were performed. The copy numbers were calculated based on a plasmid standard dilution series. Following PCR-program was used:

\begin{tabular}{ccc}
\hline Temp [ $\left.{ }^{\circ} \mathbf{C}\right]$ & Duration [s] & Cycles \\
\hline 95 & 180 & 1 \\
\hline 95 & 10 & \\
56 & 15 & 59 \\
72 & 30 & \\
\hline 25 & $\infty$ & 1 \\
\hline
\end{tabular}




\subsubsection{Quantitative NanoString nCounter multiplex analysis}

The NanoString nCounter technique represents a digital multiplexed gene expression analysis system. Reactions were prepared according to the nCounter ${ }^{\circledR}$ Gene Expression Assay User Manual for total RNA and cell lysates. $500 \mathrm{ng}$ of total RNA isolated from five embryos per stage and condition was supplied in $5 \mu \mathrm{l}$ for a total volume of $30 \mu \mathrm{l}$. The hybridization of the probe to the RNA was performed for $14-16 \mathrm{~h}$ at $65^{\circ} \mathrm{C}$. For data processing the nSolver software $\mathrm{v} 1$ and $\mathrm{v} 1.1$ program provided by NanoString Technologies was used. Counts were normalized with respect to the geometric mean of the positive controls. Furthermore, the counts were normalized with respect to the geometric mean of the housekeeping genes ornithine decarboxylase $(O D C)$ and Glucose-6-phosphate dehydrogenase (G6PDH) as these mRNAs are relatively equal expressed during the different stages of development. Background correction was done by subtracting the mean and two times the standard deviation of the counts of negative control counts for each lane. Negative values or values less than one were set to 1 as background level. Two biological independent experiments were conducted. The analyzed RNAs, target regions and sequences of the reporter probes as well as raw and processed data are shown in Appendix Figure 6.8.

\subsubsection{Whole Mount in situ hybridization (WMISH)}

In situ hybridization on whole embryos was carried out using digoxigenin-labeled antisense riboprobes as described in (Harland 1991, Hollemann and Pieler 1999). Fixed embryos were rehydrated in a graded ethanol series, washed three times in PTw and permeabilized with $10 \mu \mathrm{g} / \mathrm{ml}$ proteinase $\mathrm{K}$ in PTw for 16 minutes (stage 32 embryos). Subsequently, embryos were washed twice in $0.1 \mathrm{M}$ triethanolamine, $\mathrm{pH} 7.5$, to stop proteinase $\mathrm{K}$ digestion and acetylated by adding $25 \mu \mathrm{l}$ acetic anhydrite to fresh triethanolamine. For each step the incubation was performed for $5 \mathrm{~min}$. Thereafter, another $25 \mu \mathrm{l}$ acetic anhydrite was added. Embryos were washed 2x 5 min in PTw and refixed in PTw containing 4 \% FA for 2 min, washed five times in PTw, transferred to Hyb Mix and incubated for $5 \mathrm{~h}$ at $65^{\circ} \mathrm{C}$. Following this prehybridization step the Hyb Mix was exchanged by the digoxigenin labeled probes in Hyb Mix and incubated for $14-16 \mathrm{~h}$ at $65^{\circ} \mathrm{C}$. The next day, RNA probes were collected, embryos were washed in Hyb Mix, followed by three washing steps for $20 \mathrm{~min}$ in $2 x$ SSC at $65^{\circ} \mathrm{C}$. Non-hybridized RNA probe was removed by the addition of $20 \mu \mathrm{g} / \mathrm{ml}$ RNase $A$ and $10 \mathrm{U} / \mathrm{ml}$ RNase T1 in 2x 
SSC for $1 \mathrm{~h}$ at $37^{\circ} \mathrm{C}$. The digested probe was removed by washing once in 2x SSC at 37 ${ }^{\circ} \mathrm{C}$ for $10 \mathrm{~min}$ and twice $0.2 x \mathrm{SSC}$ at $65^{\circ} \mathrm{C}$ for $30 \mathrm{~min}$ each. Embryos were incubated two times with 1x MAB and afterwards blocked in MAB/BMB for 20 min and MAB/BMB/Horse serum for $40 \mathrm{~min}$ to minimize unspecific binding of the antibody. The antibody was applied in a 1:5000 dilution in MAB/BMB/HS and incubated for $4 \mathrm{~h}$ at RT. Thereafter, embryos were washed three times for $10 \mathrm{~min}$ with $\mathrm{MAB}$ and then incubated overnight in MAB. After three washing steps with MAB for $5 \mathrm{~min}$, the caps were exchanged and the embryos were transferred to alkaline phosphatase buffer (APB). After three washes in APB for $5 \mathrm{~min}$ each, the solution was exchanged by the color reaction solution $(\mathrm{APB}+\mathrm{NBT} / \mathrm{BCIP})$. Color reaction was carried out until the staining was nicely visible. After staining the embryos were transferred to $100 \%$ methanol to stop the reaction and remove background staining, rehydrated in methanol to 1x MEMFA and incubated for 30 min. For long time storage the MEMFA was refreshed and embryos were kept at $4{ }^{\circ} \mathrm{C}$.

\subsubsection{Bleaching of embryos}

In order to remove the pigmentation of embryos, they were washed twice in 5x SSC and transferred into bleaching solution. Bleached embryos were washed twice in 5x SSC and refixed overnight in MEMFA.

\subsubsection{DNA techniques}

\subsubsection{Plasmid DNA preparation}

For plasmid preparation of $4 \mathrm{ml}$ bacterial culture the GeneJET Plasmid Miniprep Kit was used. For plasmid preparation of $100 \mathrm{ml}$ bacterial culture the NucleoBond Xtra Midi/ Maxi was used. The plasmid DNA was eluted in $50 \mu \mathrm{R}$ Rase free $\mathrm{H}_{2} \mathrm{O}$. DNA concentrations were measured using the NanoDrop-2000c Spectrophotometer.

\subsubsection{DNA restriction digestion}

Restriction digestion was performed with endonucleases purchased from Fermentas according to the manufacturer's instructions. 


\subsubsection{Agarose gel electrophoresis}

Standard agarose gel electrophoresis was performed (Fisher and Dingman 1971). 1-2 \% (w/v) agarose gel was prepared with $1 \mathrm{x}$ TAE buffer and $0.5 \mu \mathrm{g} / \mathrm{ml} \mathrm{EtBr}$ (Sharp P. A. 1973). DNA samples were mixed with the appropriate volume of $6 x$ loading buffer (Fermentas), RNA samples were mixed with the appropriate volume of Gel Loading buffer II (Ambion) and loaded onto the gel. The gel was run at $80 \mathrm{~V}$ in 1x TAE. DNA or RNA fragments were separated in a electrical field into the agarose gel. Standard DNA ladders were used to determine the sizes of DNA/RNA fragments (High, Middle or Low Range, Fermentas). After electrophoresis, DNA/RNA bands were visualized using the UV-transilluminator and documented with the INTAS GDS documentation system.

\subsubsection{DNA fragment isolation from agarose gels or restriction digest}

The purification of DNA fragments from agarose gels or restriction digestion mixtures was performed with the Invisorb DNA CleanUp Kit.

\subsubsection{DNA sequencing}

Sequencing of 200-400 ng plasmid DNA (Sanger et al. 1992) was performed with the "Big Dye Terminator Cycle Sequencing" Kit (Applied Biosystems). The annealing temperatures were adjusted depending on the used oligonucleotide. The reaction was performed in a thermocycler with the following PCR program:

\begin{tabular}{ccc}
\hline Temp $\left[{ }^{\circ} \mathbf{C}\right]$ & Duration [s] & Cycles \\
\hline 96 & 120 & 1 \\
\hline 96 & 10 & \\
$x$ & 15 & 25 \\
60 & 240 & \\
\hline 8 & $\infty$ & 1 \\
\hline
\end{tabular}

In order to purify the amplified DNA, $1 \mu$ EDTA (125 mM), $1 \mu \mathrm{l} \mathrm{NaAc}(3 \mathrm{M})$ and $50 \mu \mathrm{l} 100$ $\%$ ethanol were added and incubated for $5 \mathrm{~min}$ at RT. The mixture was centrifuged for 20 min at $13000 \mathrm{rpm}$, the pellet was washed with $70 \%$ ethanol and centrifuged again for 5 
min at $13000 \mathrm{rpm}$. The air-dried pellet was resuspended in $15 \mu \mathrm{HiDi}$. Sequencing was performed using the ABI 3130xI Genetic Analyzer.

\subsubsection{Protein techniques}

\subsubsection{Protein isolation from embryos and oocytes}

For XDazl protein expression analysis 5 snap frozen embryos or oocytes were lysed with $50 \mu \mathrm{l}$ 1x IPP145 ColP buffer using a Micropestle (Eppendorf). For ColP analysis 50 embryos were lysed in $125 \mu$ lx IPP145 buffer. Lysates were centrifuged for 15 min at 4 ${ }^{\circ} \mathrm{C}$ and $13000 \mathrm{rpm}$, the clear protein phase was transferred into a new tube and centrifuged for another $5 \mathrm{~min}$ at $4{ }^{\circ} \mathrm{C}$ and $13000 \mathrm{rpm}$. The clear phase was either used for Co-immunoprecipitation, or samples were prepared for SDS-PAGE by addition of the appropriate amount of 2x SDS Loading buffer.

\subsubsection{Co-immunoprecipitation using embryo lysates}

For co-immunoprecipitation (CoIP) of in vivo expressed proteins, two-cell stage embryos were injected with mRNAs coding for FLAG-epitope-tagged proteins vegetally into both blastomeres and cultured until stage 12. The protein was isolated from 50 embryos, described above and a $20 \mu$ input aliquot was taken from each sample and $40 \mu \mathrm{l} 2 \mathrm{x}$ SDS Loading buffer was added. The rest of the protein lysate was incubated overnight at $4{ }^{\circ} \mathrm{C}$ with $20 \mu \mathrm{l}$ FLAG antibody coupled beads (Sigma) each, which were washed $4 \mathrm{x}$ with $800 \mu \mathrm{l}$ 1x IPP145 buffer before use. The next day, beads were centrifuged for $1 \mathrm{~min}$ at $0.8 \mathrm{rpm}$ and a supernatant fraction was taken before beads were washed again $4 \mathrm{x}$ with 1x IPP145 buffer. Every incubation and centrifugation step was carried out at $4{ }^{\circ} \mathrm{C}$. Pelleted beads were resuspended in $40 \mu \mathrm{l} 2$ x SDS Loading buffer. $20 \mu \mathrm{l}$ of each protein fraction were processed by Western Blot analysis.

\subsubsection{TNT (in vitro transcription coupled translation)}

The in vitro transcription coupled translation-assay was performed using the TNT Coupled Reticulocyte Lysate System (Promega). TNT reactions were carried out in volumes of $12.5 \mu \mathrm{l}$ using $1 \mu \mathrm{g}$ of plasmid DNA, incubated for $2 \mathrm{~h}$ at $30^{\circ} \mathrm{C}$. To test the XDazl MO efficiency $0.1-8 \mu \mathrm{g}$ XDazl MO were coincubated in the TNT. The proteins were analyzed by Western Blot analysis. 


\subsubsection{Co-immunoprecipitation with in vitro translated proteins and Cy3-labeled RNAs}

In vitro RNA-protein interactions were analyzed using co-immunoprecipitation (ColP) with in vitro translated FLAG-epitope tagged proteins and in vitro transcribed Cy3-labeled RNA. Bound RNAs were analyzed on a denaturing urea gel.

1x Reaction mix for RNA-protein binding:

$\begin{array}{ll}10 \mu \mathrm{l} & \text { TNT translated protein } \\ \mathrm{x} \mu \mathrm{l} & \text { Cy3-labeled RNA }(40 \mathrm{ng}) \\ 2 \mu \mathrm{l} & 5 \mathrm{x} \text { UV crosslink buffer } \\ 1 \mu \mathrm{l} & \text { Yeast RNA }(5 \mathrm{mg} / \mathrm{ml}) \\ \underline{\mathrm{x}} \mu \mathrm{l} & \text { RNase free } \mathrm{H}_{2} \underline{\mathrm{O}}\end{array}$

$20 \mu \mathrm{l}$

Cy3-labeled RNAs were mixed and added to the reaction mix. An input aliquot of $0.5 \mu \mathrm{l}$ Cy3-RNA was taken before addition to the reaction and mixed with Gel Loading Buffer II (Ambion, mMassage mMachine Kit). The binding reaction was performed for $30 \mathrm{~min}$ at $\mathrm{RT}$ in the dark. After the binding reaction, $20 \mu \mathrm{l}$ washed anti-FLAG sepharose beads and $500 \mu \mathrm{l}$ YSS buffer were added for the co-Immunoprecipitation, which was carried out for $2 \mathrm{~h}$ at $4{ }^{\circ} \mathrm{C}$. Thereafter, the fractions were centrifuged for $1 \mathrm{~min}$ at $0.8 \mathrm{rpm}$. A $200 \mu \mathrm{l}$ supernatant aliquot was taken, $20 \mu \mathrm{l} 10 \%$ SDS as well as $1 \mu \mathrm{l}$ glycogen were added to the aliquot and the fraction was supplied to RNA extraction. Beads were washed $4 x$ in $800 \mu \mathrm{l}$ YSS buffer, centrifuged for $1 \mathrm{~min}$ at $0.8 \mathrm{rpm}$ and the buffer was removed. Beads were resuspended in $200 \mu \mathrm{l}$ YSS buffer and $20 \mu \mathrm{l} 10 \%$ SDS as well as $1 \mu$ l glycogen were added. Three RNA extraction steps followed using 1 volume Roti-Aqua-Phenol/C/I (Roth), 1 volume 24/1 Chloroform/Isoamyl alcohol and 0.2 volumes $5 \mathrm{M}$ ammonium acetate as well as 5 volumes of $100 \%$ ethanol. Between each step, samples were centrifuged for $10 \mathrm{~min}$ at $4{ }^{\circ} \mathrm{C}$ and $13000 \mathrm{rpm}$. After another washing step using $80 \%$ ethanol the air-dried RNA pellet was dissolved in $10 \mu \mathrm{l}$ Gel Loading Buffer II for the supernatant and $5 \mu \mathrm{l}$ for the bead pellet fraction and analyzed on a urea gel. Proteins from the interphase and the phenol phase $(200 \mu \mathrm{l})$ were taken after purification with 1 volume Roti-Aqua-Phenol/C/l (Roth). This fraction was mixed with $1 \mathrm{ml}$ ice-cold acetone and precipitated overnight at $-20^{\circ} \mathrm{C}$. The next day, the protein fractions were centrifuged for $30 \mathrm{~min}$ at $13000 \mathrm{rpm}$ at $4{ }^{\circ} \mathrm{C}$, pellets were washed with $75 \% \mathrm{EtOH}$ and centrifuged 
for 5 min at $4{ }^{\circ} \mathrm{C}$. The air-dried pellet was dissolved in $10 \mu \mathrm{l} 2 \mathrm{x}$ SDS Loading buffer and prepared for SDS-PAGE.

\subsubsection{Urea-PAGE electrophoresis}

The urea gel mixture was prepared as described below and poured into mounted glass plates with $1 \mathrm{~mm}$ spacers. RNA samples in Gel Loading buffer II were heated to $95{ }^{\circ} \mathrm{C}$ for $2 \mathrm{~min}$, incubated on ice for $3 \mathrm{~min}$ and loaded on a pre-run gel (10 $\mathrm{min}$ at $150 \mathrm{~V}$ ). The gel was run at $250 \mathrm{~V}$ up to the required distance. After the run, the gel was analyzed for the Cy3-labeled RNAs with the Typhoon imager using the ImageQuant software.

\section{$\underline{7 \% \text { urea stock solution }}$}

$\begin{array}{ll}40 \% \text { Acrylamide (38:2) } & 4.38 \mathrm{ml} \\ \text { Urea } & 10.51 \mathrm{~g}(7 \mathrm{M}) \\ 10 x \text { TBE } & 2.5 \mathrm{ml} \\ 10 \% \text { APS } & 300 \mu \mathrm{l} \\ \text { TEMED } & 30 \mu \mathrm{l} \\ \mathrm{H}_{2} \mathrm{O} & \text { to } 25 \mathrm{ml}\end{array}$

\subsubsection{Electrophoretic Mobility Shift Assay (EMSA)}

This method was carried out based on the protocol of Chung and colleagues (Chung et al. 1996). EMSA reaction mixtures were set up as indicated below and incubated at RT for $30 \mathrm{~min}$ in the dark. To test the effects of XDazl-LE MOs, $0.75 \mu \mathrm{l}$ of the MO $(1 \mathrm{mM})$ was added to each EMSA reaction mix. EMSA loading dye $(5 \mu \mathrm{l})$ was added after the incubation for $30 \mathrm{~min}$ and the samples were loaded on a $1 \%$ agarose gel. The agarose gel was prepared in an EtBr-free chamber and run in 1x TBE at $40 \mathrm{~V}$ for $\sim 1.5$ hours. The gel was scanned with the Typhoon 9400 in fluorescence mode (Cy3, Alexa546).

EMSA reaction mix:

$\begin{array}{ll}\text { 5x Crosslink buffer } & 4 \mu \mathrm{l} \\ \text { Recombinant protein } & 1 \mu \mathrm{l}(0-2.5 \mu \mathrm{M} \text {, up to maximum protein concentration) } \\ \text { Cy3-RNA } & 1 \mu \mathrm{l}(0.4 \mathrm{pmol}) \\ \text { Yeast RNA } & 1 \mu \mathrm{l} \\ \text { RNase free } \mathrm{H}_{2} \mathrm{O} & \text { add to } 20 \mu \mathrm{l}\end{array}$


The background correction was performed using the ImageQuant 5.2 software. Resulting values were further processed in Microsoft Excel 2010 to calculate the percentage of bound and unbound RNA. In the Graph Pad Prim 6 software the $K_{D}$ values for the binding efficiencies were calculated.

\subsubsection{SDS-PAGE protein electrophoresis}

SDS polyacrylamide gels were prepared according to the protocol of Laemmli (Laemmli 1970) for the analysis of proteins in Western Blot or Coomassie Blue staining. SDS gels $(10-12 \%)$ were made depending on the molecular weight of the analyzed proteins. At first, the resolving gel was poured and allowed to polymerize with a layer of $0.1 \%$ SDS on top. After the resolving gel had polymerized, the stacking gel solution was poured and a plastic comb was inserted to mold the pockets for loading samples. Samples were loaded onto the gel along with PageRuler Plus Prestained Protein Ladder (Fermentas) for Western Blot analysis or with the Unstained SDS-PAGE standard Broad Range (BioRad) for Coomassie Blue staining. The gel run was performed in 1x Laemmli running buffer at $80 \mathrm{~V}$ for the stacking gel and $180 \mathrm{~V}$ for the resolving gel.

\subsubsection{Western Blot Analysis}

After SDS-PAGE, proteins were transferred to a Propan Nitrocellulose membrane $(0.45$ $\mu \mathrm{m}$, Whatman) (Towbin et al. 1979) using a semi-dry blotting technique. Blotting was carried out in Protein Blotting buffer (semi dry) for $1 \mathrm{~h}$ applying $40 \mathrm{~V}$ and was set up with the protein gel placed on nitrocellulose between layers of three filter papers (Whatman) soaked in Protein Blotting Buffer. After blotting, the membrane was incubated for $1 \mathrm{~h}$ at RT in Blocking solution. After blocking, specific primary antibodies were applied diluted to the appropriate concentration in Blocking buffer and incubated on a shaking table overnight at $4{ }^{\circ} \mathrm{C}$. The next day, the membrane was washed three times for $10 \mathrm{~min}$ each, with TBST at RT. Horseradish peroxidase (HRP) coupled or fluorescent IRDyes coupled (LI-COR) secondary antibodies in blocking buffer were added and incubated at RT for 1 h. The membrane was washed three times for 10 min each, with TBST at RT. HRPactivity was detected using the ECL Kit SuperSignal West Dura (Pierce) on X-ray detection films (Amersham). Fluorescent signals were detected using the LI-COR Odyssey Infrared Imaging system. 


\subsubsection{Coomassie Blue Staining}

The procedure was performed according to an online available protocol from Roger Rowlett, Colgate University for rapid ethanol-based Coomassie Blue staining of SDS-

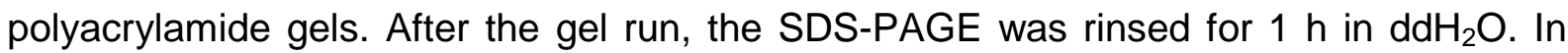
between the water was changed for three times. After rinsing, Coomassie staining solution was added. The gel was heated until the solution just begins to boil. Gel was incubated in the Coomassie staining solution for 15-60 minutes until the bands were detectable. The staining solution was removed and the gel was rinsed with water to remove excess of staining solution. Coomassie destaining solution was added. The gel was destained until the background was removed. Destained gels were rinsed thoroughly with and stored in distilled water.

\subsubsection{Recombinant protein expression and purification}

For recombinant protein expression according to Baneyx (Baneyx 1999) BL21 (DE3) cells were used for transformation with the expression vector containing the appropriate insert (XDE ORF, XDazl RRM). A single colony was picked and inoculated in $50 \mathrm{ml}$ LB + antibiotic at $37^{\circ} \mathrm{C}$ overnight. Next morning the culture was diluted $1: 50$ or $1: 100$ in a $1.5 \mathrm{I}$ $\mathrm{LB}+$ antibiotic (OD 0.05) containing Erlenmeyer flask and incubated at $37^{\circ} \mathrm{C}$ shaking at $200 \mathrm{rpm}$. At $\mathrm{OD}_{600} \sim 0.4-0.51 \mathrm{mM}$ (final concentration) of IPTG was added to the culture and the culture was incubated at $28{ }^{\circ} \mathrm{C}$ for $4.5 \mathrm{~h}$. Before IPTG addition, a $0.5 \mathrm{ml}$ aliquot was taken, pelleted and 2x SDS Loading buffer was added for analyzing bacterial protein pattern in uninduced cells on a SDS-PAGE. The pellet in SDS Loading buffer was stored at $-20^{\circ} \mathrm{C}$. In between and after the $4.5 \mathrm{~h} 0.5 \mu \mathrm{l}$ samples were taken, pelleted and stored to monitor the increase of protein expression. The cultures were pelleted at $6000 \mathrm{rpm}$ and pellets were stored at $-20^{\circ} \mathrm{C}$ for protein isolation. Aliquots, taken in between, were analyzed on a $12 \%$ SDS-PAGE followed by Coomassie staining to test for protein expression.

\section{His-tagged protein purification for XDE (pETM30)}

The cell pellet was resuspended in $25 \mathrm{ml}$ HNTA buffer and complete lysis of the bacteria cells was achieved in a Fluidizer system. Following centrifugation for 20 min at 10000 rpm, the soluble fraction was mixed with $2 \mathrm{ml}$ of MNTA-equilibrated Ni-NTA resin (Qiagen) and rotated at $4{ }^{\circ} \mathrm{C}$ for $2 \mathrm{~h}$ to allow protein binding. The protein-resin solution 
was transferred on a Poly-Prep Chromatography Column (Bio-Rad), washed 3x with HNTA buffer $+20 \mathrm{mM}$ imidazole and $3 x$ with MNTA buffer $+20 \mathrm{mM}$ imidazole. Elution was performed with MNTA buffer containing increasing amounts of imidazole (50 - 1000 $\mathrm{mM}$ ). The protein was dialyzed against $1 \mathrm{x}$ buffer $\mathrm{M}$ and stored at $-80^{\circ} \mathrm{C}$.

\section{GST-tagged protein purification for XDazl (pGEX-6p3):}

The cell pellet was resuspended in $50 \mathrm{ml}$ High Salt-PBS (HS-PBS) and complete lysis of the bacteria cells was achieved in a Fluidizer. Following centrifugation for 30 min at 15 $000 \mathrm{rpm}$, the soluble fraction was mixed with $4 \mathrm{ml}$ of HS-PBS-equilibrated GT-sepharose 4 Fast Flow resin (GE Healthcare) and rotated at $4{ }^{\circ} \mathrm{C}$ for $2 \mathrm{~h}$ to allow protein binding. The protein-resin solution was transferred on a Poly-Prep Chromatography Column (BioRad), washed $4 x$ with HS-PBS. Elution of the beads was performed with HS-PBS containing $15 \mathrm{mM}$ Glutathione. The protein was dialyzed against 1x buffer $\mathrm{M}$ and stored at $-80^{\circ} \mathrm{C}$.

\subsubsection{Bradford Assay}

Bradford assay was performed in principle after Bradford (Bradford 1976). Bradford stock solution was prepared by dissolving $100 \mathrm{mg}$ Coomassie Brilliant Blue G-250 in $50 \mathrm{ml} 95$ $\%$ ethanol and $100 \mathrm{ml} 85 \%$ (w/v) phosphoric acid. When the dye was completely dissolved, the volume was increased to 1 liter and the solution was filtered through Whatman paper. The Bradford Working buffer was stored in the dark at $4{ }^{\circ} \mathrm{C}$ for up to 6 months. A standard curve was made using 0 - $20 \mu \mathrm{g}$ BSA to determine the concentration factor. Measurement of protein concentration was performed by adding increasing amounts $(2.5-25 \mu \mathrm{l})$ of the sample to $1 \mathrm{ml}$ of the Bradford reagent and thoroughly mixing in cuvettes. Sample measurements were performed in triplets. After incubation at RT for $5 \mathrm{~min}$, the OD was measured at $595 \mathrm{~nm}$ (NanoDrop). The protein concentration was estimated using the concentration factor from the standard curve.

\subsubsection{Immunofluorescence staining of oocytes}

Cy3-labeled RNA injected oocytes in 1x MBS were applied to immunofluorescence staining according to the protocol of J. Gagnon and K. Mowry (Gagnon and Mowry 
2011). In contrast to the protocol, the oocytes were directly applied to Proteinase $\mathrm{K}$ digest without fixation in MEMFA.

Primary antibody: $\quad$ Mouse anti-XDazl, 1:500 (Mita and Yamashita 2000)

Secondary antibody: $\quad$ Goat anti-mouse Alexa Fluor 633 (Invitrogen)

The oocytes were analyzed using the Zeiss LSM780 and the ZEN software 2011.

\subsubsection{Xenopus embryo culture and microinjections}

\subsubsection{Embryo culture and microinjections}

In female Xenopus laevis frogs oviposition was induced by injection of 1000 units human chorionic gonadotropin (hCG, Sigma Aldrich) into the dorsal lymph sac, approximately 16 hours before supposed egg-laying. Frogs were kept at $16^{\circ} \mathrm{C}$ overnight. Laid eggs were in vitro fertilized with minced testis in $0.1 \times$ MBS. Fertilized eggs were treated with $2 \%$ cysteine hydrochloride, $\mathrm{pH} 7.8$ - 8.0, to remove the jelly coat. The embryos were cultured in $0.1 \times \mathrm{MBS}$ at $12.5-18^{\circ} \mathrm{C}$. Injections were performed in injection buffer on a cooling plate $\left(12.5-16^{\circ} \mathrm{C}\right)$. For microinjections, the solutions were loaded into the glass needles (Science Products, GB 100F-8P) prepared on a needle puller. For different purposes, injections were performed at two-cell stage of embryogenesis, $4.2 \mathrm{nl}$ of the appropriate solution were injected per blastomere. After injection, embryos were kept for at least 1 hour in the injection buffer to allow the healing and were transferred into $0.1 \mathrm{x}$ MBS afterwards. The developmental stages were defined according to Nieuwkoop and Faber (Nieuwkoop 1994). At the appropriate stage the embryos were fixed in liquid $\mathrm{N}_{2}$ for protein and RNA isolation or in 1x MEMFA for whole mount in situ hybridization. For WMISH the embryos were dehydrated in $\mathrm{EtOH}$ and stored in $100 \% \mathrm{EtOH}$.

For the Xenopus laevis/tropicalis hybrid generation the $X$. laevis females were injected with of 1000 units human chorionic gonadotropin (hCG, Sigma Aldrich) into the dorsal lymph sac, approximately 16 hours before supposed egg-laying. $X$. tropicalis male was injected with 20 units human chorionic gonadotropin (hCG, Sigma Aldrich) into the dorsal lymph sac approximately 16 hours before testes preparation. The next day, the male was narcotized and sacrificed by submerging it in $0.25 \% 3$-aminobenzoic methanesulfonate solution for 20 - $30 \mathrm{~min}$ at room temperature. The frog was decapitated and testes were removed. Testes were washed three times with $1 \mathrm{x}$ MBS, the surrounding blood vessels were removed and minced to fertilize $X$. laevis eggs. The embryos were grown at $12{ }^{\circ} \mathrm{C}$ until the appropriate stage was achieved. Then they were fixed in liquid $\mathrm{N}_{2}$ and supplied to RNA extraction. 


\subsubsection{Preparation of Xenopus laevis testis}

The Xenopus laevis male frog was narcotized and sacrificed by putting it in $0.25 \% 3$ aminobenzoic methanesulfonate solution for 20 - $30 \mathrm{~min}$ at room temperature. The frog was decapitated and testes were removed. Testes were washed three times with $1 \mathrm{x}$ MBS, the surrounding blood vessels were removed and testes were stored in $1 \mathrm{x}$ MBS buffer at $4{ }^{\circ} \mathrm{C}$ for $\sim 1$ week.

\subsubsection{Oocyte culture and microinjection}

Oocytes were isolated from adult, female Xenopus laevis via operation on ice under anesthesia with $0.25 \%$ 3-aminobenzoic methanesulfonate solution (20 min anesthesia). Separation of the oocytes was achieved by treatment with $1 \mathrm{mg} / \mathrm{ml}$ liberase blendzyme (Roche) in collagenase-buffer and shook carefully up to $120 \mathrm{~min}$. Oocytes were washed using $1 \mathrm{x}$ MBS until the buffer was clear, incubated at $18{ }^{\circ} \mathrm{C}$ and staged according to Dumont (Dumont 1972). Oocytes were injected in 1x MBS using the appropriate injection solution in $2.1 \mathrm{nl}$ for stage I-III and $4.2 \mathrm{nl}$ for IV-VI oocytes. The same materials were used like for embryo injection. The Cy3-labeled RNA was diluted to a concentration of 75 $\mathrm{ng} / \mathrm{\mu l}$, before injection. Injected oocytes were cultivated for 3 days in oocyte culture medium (OCM) at $18{ }^{\circ} \mathrm{C}$, rinsed in $1 \mathrm{x}$ MBS before they were transferred to $1 \mathrm{x}$ MBS for immunofluorescence staining. For protein isolation staged, uninjected oocytes were fixed in liquid $\mathrm{N}_{2}$ and applied to protein isolation. 


\section{RESULTS}

\subsection{XDazl mediates protection of germ cell specific mRNAs in Xenopus embryos}

\subsubsection{XDazl stabilizes germ cell specific reporter and endogenous mRNAs in vivo}

During embryonic development, the establishment of a functional reproductive system is of major importance. In this process, the segregation of the future germline from the somatic tissue is crucial. Germ cell specific transcripts, carrying the so-called localization element (LE) in their 3' untranslated region (UTR), are stabilized in PGCs while depleted in somatic cells (Kloc et al. 2002, Mishima et al. 2006). Correspondingly, Koebernick and colleagues could show that somatic clearance of the germ cell specific Xenopus Dead end-LE reporter (XDE-LE-R) RNA is achieved by Xenopus miR-18 targeting. In germ cells this reporter RNA is protected from somatic decay by the cooperative activity of ElrB1 and XDE proteins counteracting the degradation process (Koebernick et al. 2010). However, it is unknown whether additional proteins are involved in the mechanism of germ cell specific mRNA protection. We hypothesized XDE and XDazl may play a similar role in stabilizing germ cell specific mRNAs as knockdown of both, XDE and XDazl, leads to a similar phenotype, a reduction if not the loss of PGCs (Houston and King 2000a, Horvay et al. 2006). In order to determine if XDazl protein indeed counteracts somatic degradation, GFP-XDE-LE reporter RNA, consisting of the GFP open reading frame (ORF) and the Xenopus Dead end LE, was coinjected with XDaz/mRNA. Injection into the vegetal hemisphere of the Xenopus embryo ensures the enrichment of the reporter RNA in the germ plasm; the LE is responsible for reporter RNA degradation in somatic endodermal cells (Koebernick et al. 2010). XDazl overexpression indeed results in somatic stabilization of XDE-LE-R RNA (Figure 3.1 A). Reporter injection alone leads to reporter presence most prominently in PGCs, while only a moderate fraction of these embryos exhibited weak or strong somatic GFP-XDE-LE stabilization. Low coinjected concentrations of XDazl mRNA (25 or $50 \mathrm{ng}$ ) did not significantly alter reporter RNA stability. However, upon coinjection of higher XDazl amounts the presence of the reporter in the endoderm increased (Figure $3.1 \mathrm{~B}$ ). Interestingly, the injection of XDazl mRNA concentrations equal or higher than $100 \mathrm{pg}$ is enough to saturate the system. We can conclude that XDazl has a stabilizing function on the XDE-LE reporter RNA in a dose dependent manner. 
A
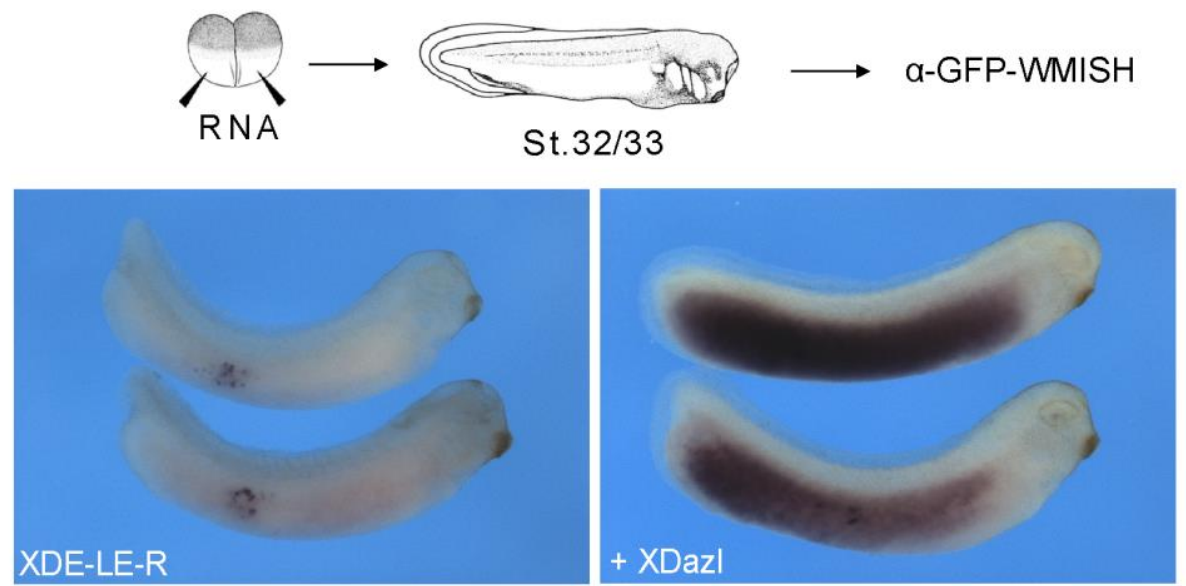

B
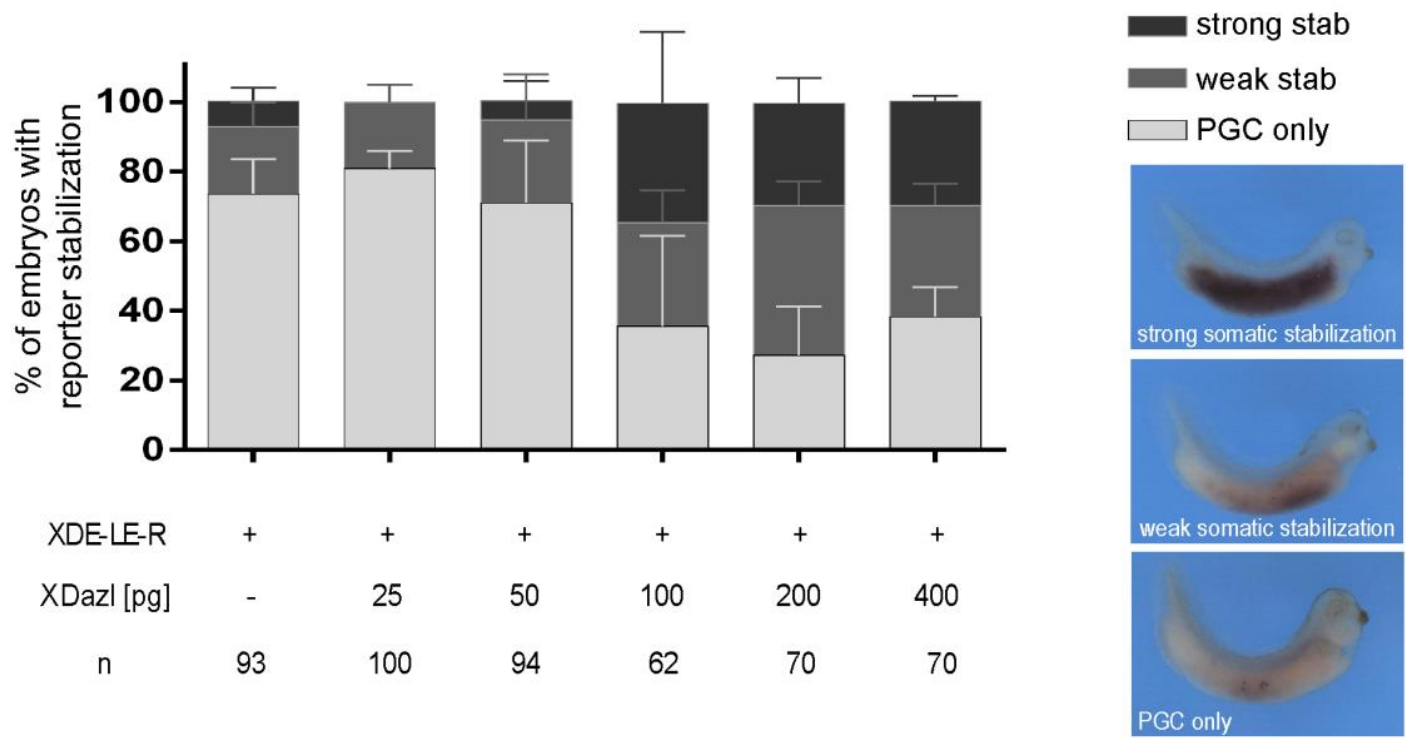

Figure 3.1. Ectopic XDazl protein expression protects XDE-LE reporter RNA from somatic clearance in a dose dependent manner.

Embryos were injected vegetally at two-cell stage with $500 \mathrm{pg}$ of GFP-XDE-LE reporter RNA alone or together with XDazl mRNA and cultured until stage 32. Reporter RNA presence was determined by WMISH using a probe against GFP.

A) XDazl protein stabilizes the $X D E-L E$ reporter RNA in somatic cells. Embryos were injected with 500 pg of $X D E$ $L E$ reporter RNA alone or together with $200 \mathrm{pg}$ of $X D a z l$ mRNA. Strong somatic stabilization of $X D E-L E-R$ was detectable after coinjection in subpopulations of the embryo.

B) XDazl protein induces somatic $X D E-L E$ reporter stabilization in a dose dependent manner. Embryos were injected vegetally (2/2 cells) with $500 \mathrm{pg} X D E-L E-R$ alone or together with 25, 50, 100, 200 or $400 \mathrm{pg} X D$ azl mRNA. WMISH stained embryos were ranked by eye into three classes: PGC only, weak somatic stabilization, strong somatic stabilization. Right panel indicates representative embryos showing the observed phenotypes. Left panel shows the quantification of observed somatic reporter RNA levels (\%) scored in injected embryos. The graphs summarize the results of three independent experiments. Error bars indicate standard errors. $n$, number of analyzed embryos.

Further, we wanted to examine whether the protective activity of XDazl is also observed for other known germ cell specific mRNAs. For this purpose, the same experimental setup was used as described above for other germline LE-reporter RNA constructs. In absence of XDazl protein, the reporter RNAs for Xcat2-, Xpat- and XDazI-LE were detected predominantly in the PGCs (Figure 3.2 A, B). However, when coinjected with $X D a z l$ mRNA, somatic stabilization of all three reporters was significantly increased 
(Figure 3.2 A, B). Additionally, we investigated the influence of ectopic XDazl expression on reporter RNA levels by quantitative RT-PCR (Figure $3.2 \mathrm{C}$ ). To address this issue, total mRNA of stage 32 injected embryos was isolated and evaluated. Consistent with the results from WMISH analyses, we found that XDazl protein presence leads to increased reporter levels for all three germ cell specific RNAs analyzed, Xcat2-, Xpatand the XDazI-LE. Taken together, XDazl has a general activity in LE-mediated stabilization of the reporter RNAs containing LEs from germ cell specific mRNAs. 
A
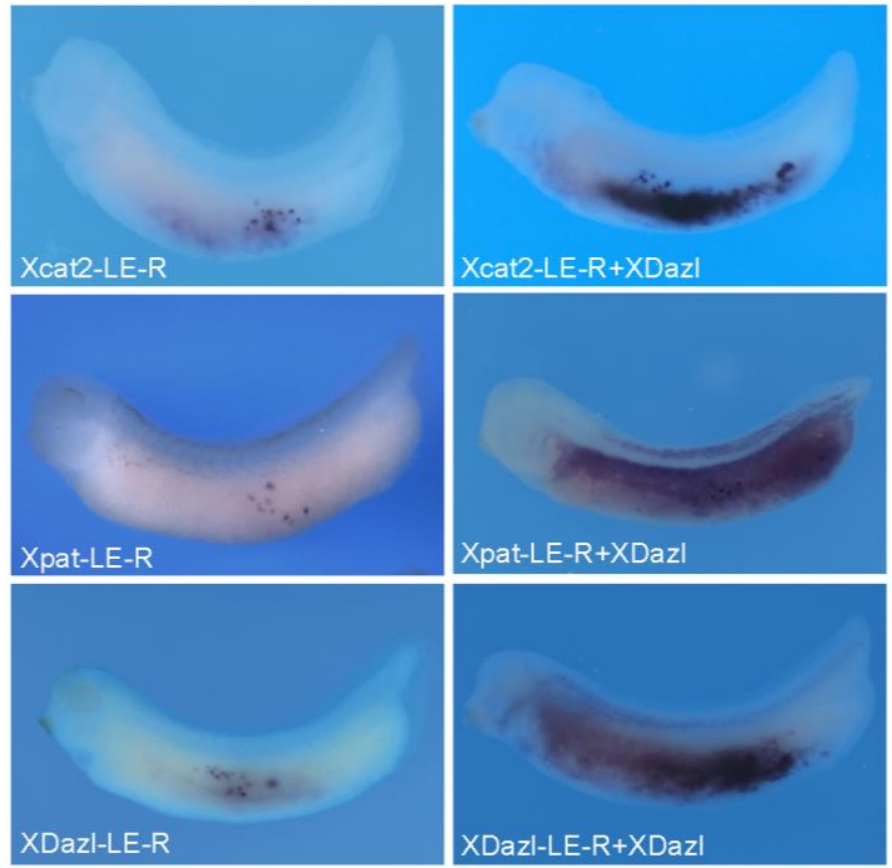

B

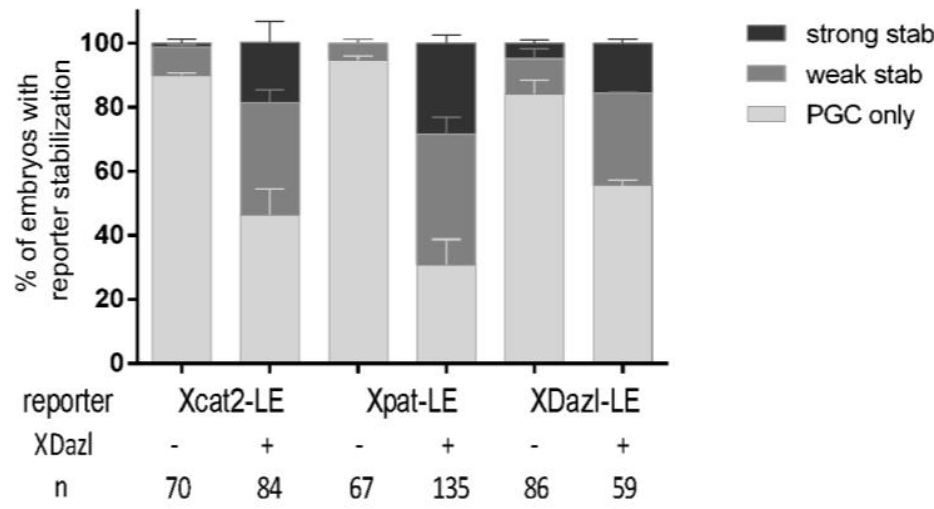

C

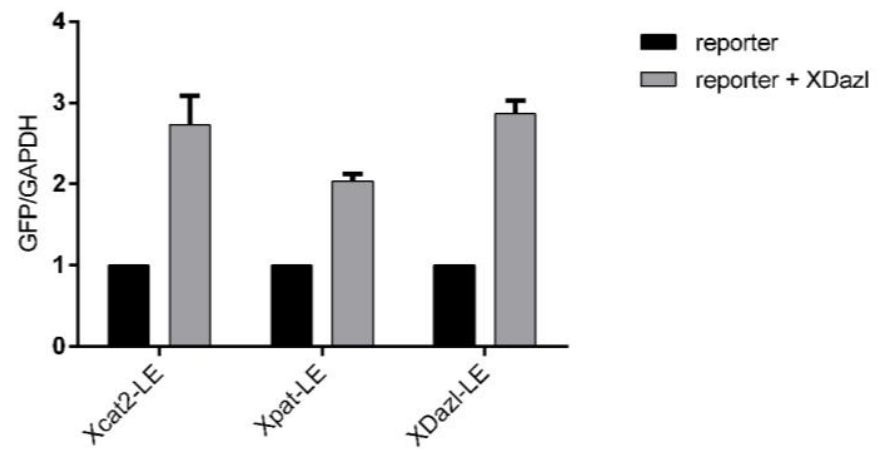

Figure 3.2. Ectopic expression of XDazl in Xenopus embryos has a stabilizing effect on multiple germ cell specific RNAs.

Both blastomeres of two-cell-stage embryos were injected vegetally with $800 \mathrm{pg}$ of reporter RNA for the different LEs alone or together with $100 \mathrm{pg}$ of XDazl mRNA and cultured until tadpole stage.

A+B) XDazl protein presence stabilizes reporter RNAs of Xcat2-, Xpat-, and XDazI-LE in somatic cells. Reporter RNA presence was determined by WMISH using a probe against GFP.

Embryos injected with the reporter RNA alone showed presence of the reporter mainly in the PGCs. Coexpression of XDazl led to strong somatic stabilization of the reporter RNAs.

B) Comparative diagram of somatic reporter RNA levels (\%) scored in injected embryos. The graph summarizes the results of three independent experiments. Error bars represent standard errors. n, number of analyzed embryos.

C) Quantitative RT-PCR analyzing the GFP-reporter presence normalized to the housekeeping gene GAPDH in reporter only injected embryos compared to reporter $+X D a z l$ injected embryos at stage 32 . 
We further addressed the question, whether ectopic expression of XDazl has also an effect on endogenous germ cell specific mRNAs. For this purpose, embryos were injected vegetally with the XDE-LE reporter RNA alone or coinjected with XDazl at twocell stage and cultivated until stage 10.5. Profiling of the transcript levels for embryos in each condition was performed using NanoString nCounter multiplex analysis (Figure 3.3 A). We found XDazl to have a stabilizing effect on all endogenous germ cell specific mRNAs analyzed in this study (Figure 3.3 B, Appendix 6.8.2). For the germ cell specific mRNAs cyclin A1, cyclin B2, XDazI_UTR, XDeadSouth, Xcat2 and Xpat a two-fold increase in expression could be detected, while $X D E$ coding sequence (XDE_CDS) and germes mRNA were enriched even three-fold (Figure $3.3 \mathrm{~B}$ ). Non-germ cell specific mRNAs, such as B-actin and laminB1, were not affected by ectopic XDazl expression (Figure 3.3B). These data led us to the assumption that XDazl specifically stabilizes germ line transcripts.

A

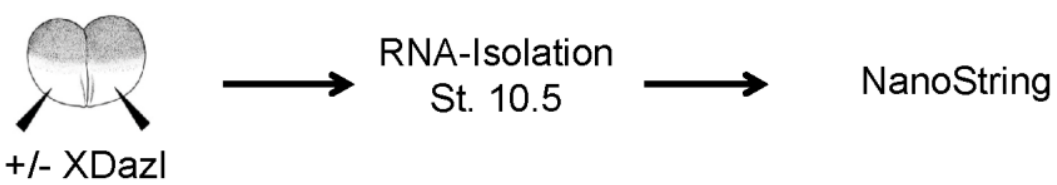

B

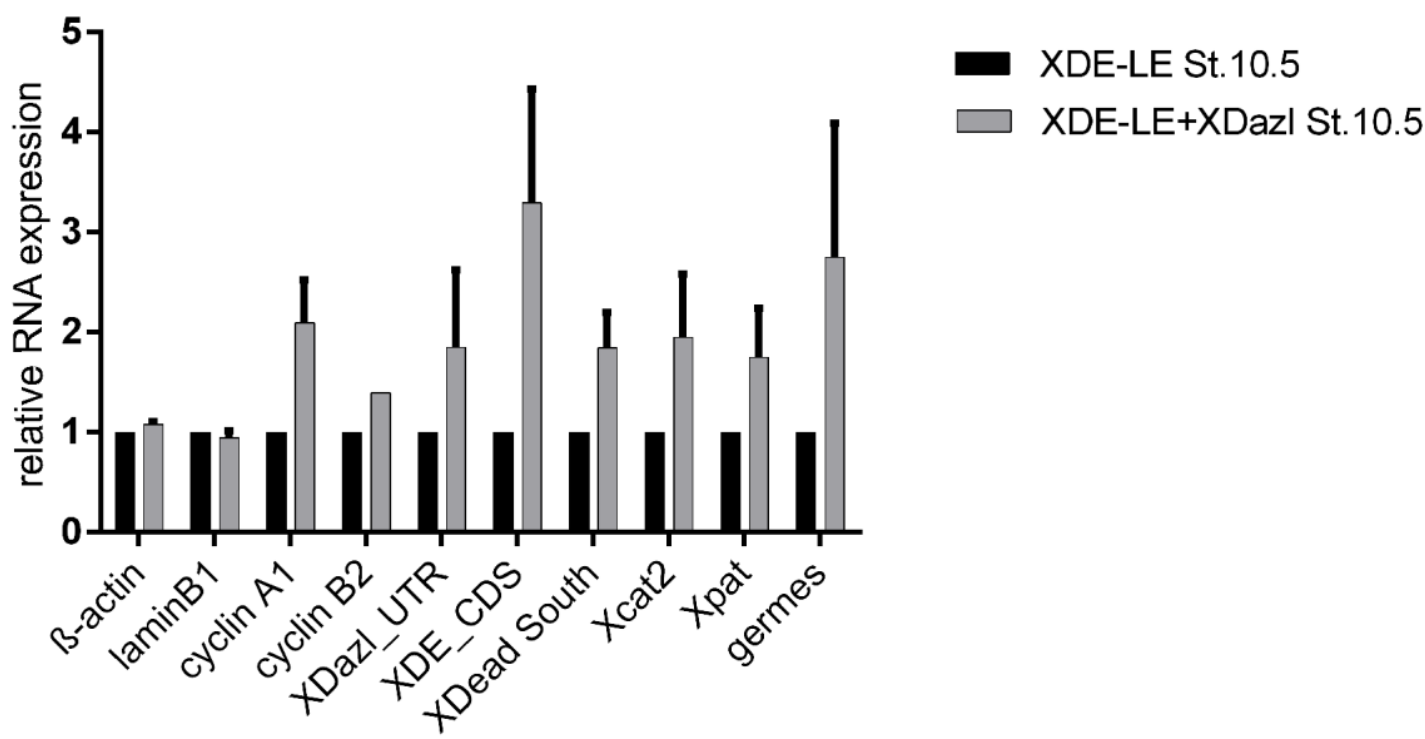

Figure 3.3. XDazl stabilizes endogenous germ cell specific mRNAs.

$\mathrm{A}+\mathrm{B})$ Xenopus embryos were injected vegetally into both blastomeres at two-cell stage with XDE-LE- $R$ RNA. In a second cohort of embryos 200 pg XDazl mRNA, encoding the XDazl ORF, was coinjected. Total mRNA was extracted from five embryos for both experimental cohorts at stage 10.5 of embryogenesis. The transcript levels were analyzed via NanoString nCounter multiplex analysis.

B) The graph shows the averaged fold changes over XDE-LE reporter RNA only injected embryos from two independent experiments for different germ cell specific mRNAs. The values for XDE-LE only injected embryos were set to 1. Error bars represent the standard deviation. 
The mechanism of XDazl mediated stabilization was not yet determined in Xenopus laevis. As the XDazl protein contains a highly conserved RNA-recognition motif (RRM), which includes two ribonucleoprotein (RNP) domains, we asked whether these are required for mRNA stabilization. To this end, deletion constructs of the XDazl ORF, lacking either one or both RNP domains, were generated (Figure 3.4 A). Transcripts encoding these deletion mutants were injected vegetally together with $X D E-L E$ reporter RNA into two-cell stage embryos. As a positive control XDazl wild-type transcript was coinjected with the $X D E-L E-R$. Analysis of samples by WMISH indicated that the GFPreporter is strongly stabilized in somatic endodermal cells of tadpole embryos for the overexpression of wild-type XDazl, as above. The loss of either RNP1 or RNP2 was sufficient to lose the protective function of XDazl on the reporter RNA in the soma (Figure 3.4 B, C). Consequently, these data show that the XDazl RNPs are both essential for the mRNA stabilizing function. 
A

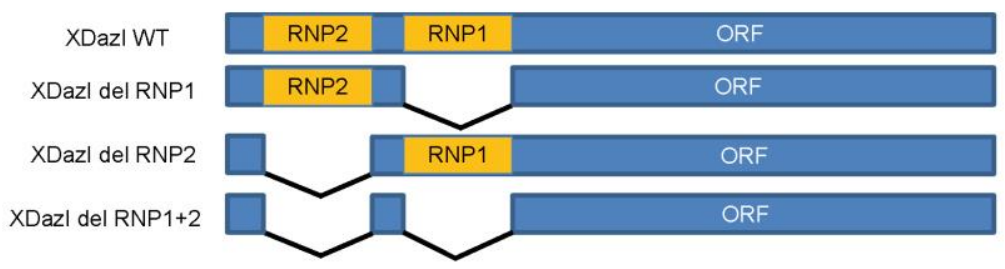

B
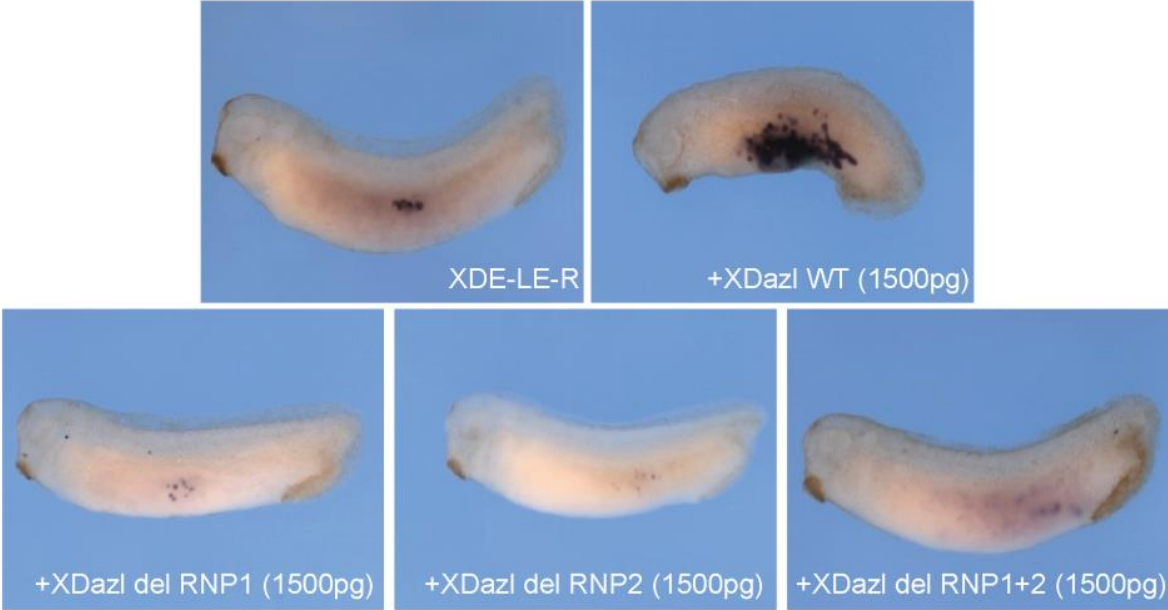

C

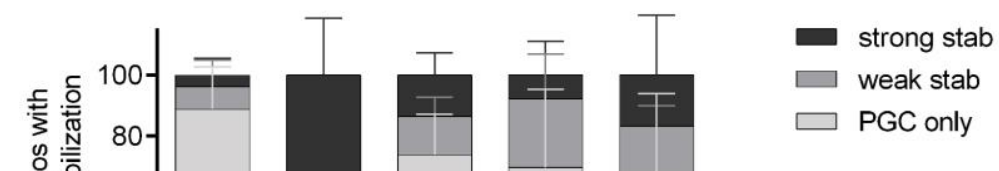

Figure 3.4. The XDazl RNA recognition motif is necessary for the stabilizing function of the XDazl protein.

A) Scheme representing the XDazl ORF constructs employed for the analysis of XDazl RRM function.

Beside the wild-type construct (XDazl WT) containing the full XDazl ORF with the RRM, which is composed of two characteristic RNPs, deletion constructs with only one RNP (XDazl del RNP1, XDazl del RNP2) or the deletion of both RNPs (XDazl del RNP1+2) were generated.

$\mathrm{B}+\mathrm{C})$ 2-cell stage embryos were injected vegetally into both blastomeres with $500 \mathrm{pg} X D E-L E$ reporter RNA alone or together with $1500 \mathrm{pg}$ of either XDazl WT, XDazl del RNP1, XDazl del RNP2 or XDazl del RNP1+2 mRNA. At tadpole stage 32 the embryos were fixed, WMISH against the reporter was performed and the embryos were analyzed for somatic reporter stabilization. Embryos were stained in parallel for the same time.

B) Representative embryos are depicted.

C) Comparative diagram of somatic reporter RNA levels (\%) scored in injected embryos. The graph summarizes the results of two independent experiments. Error bars represent the standard deviation. n, number of analyzed embryos. 
The stabilization of germline specific transcripts validated by NanoString nCounter multiplex analysis and somatic presence of reporter constructs observed in the whole mount embryos overexpressing XDazl provide strong indications for the involvement of the XDazl protein in germline mRNA protection during the process of PGC-segregation from the surrounding somatic tissue. Additionally, the RNA recognition motif was shown to be essential for the stabilizing function of XDazl.

\subsubsection{XDazl functions together with XDE and ElrB1 proteins in somatic XDE-LE reporter RNA stabilization}

RNAs are regulated by incorporation into RNP complexes, containing multiple proteins. In the case of XDE, it is known to associate with ElrB1 in a RNP complex responsible for the protection of $X D E-L E$ in the germline. The synergistic function of both proteins protects $X D E-L E$ from miR-18 mediated somatic degradation (Koebernick et al. 2010). However, it remains unclear if further proteins are cooperating with XDE or ElrB1 to promote this protective function. Hence, we asked whether XDazl could function in a synergistic manner with XDE as well. To answer this question, XDE-LE reporter RNA was coexpressed with subcritical amounts of mRNA encoding for XDE and/or XDazl proteins (Figure 3.5 A). Under these conditions, overexpression of only one protein, XDE or XDazl, did not or only weakly stabilize the $X D E-L E$ reporter RNA in the soma. In contrast, injection of the same quantities of $X D a z l$ and $X D E$ mRNA along with the $X D E$ $L E$ reporter RNA results in significant somatic reporter RNA stabilization in tadpole stage embryos (Figure 3.5 B). These data suggest that XDazl and XDE proteins cooperate to protect germ cell specific mRNAs from somatic clearance. 
A
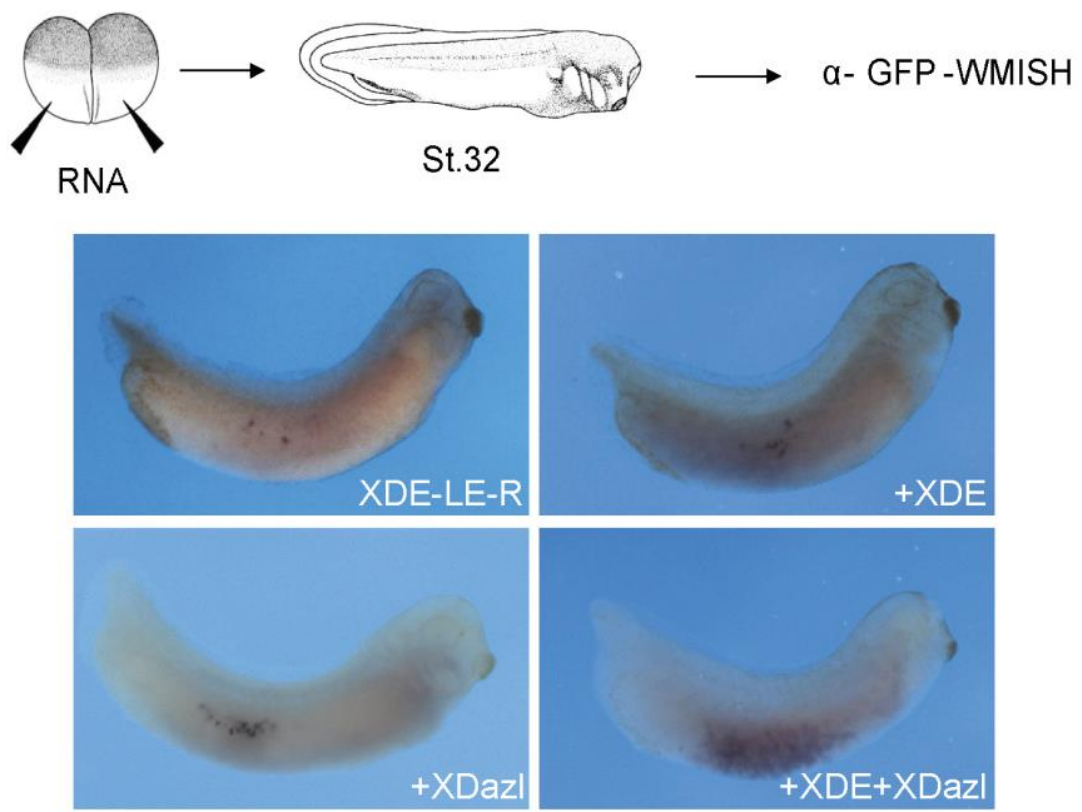

B

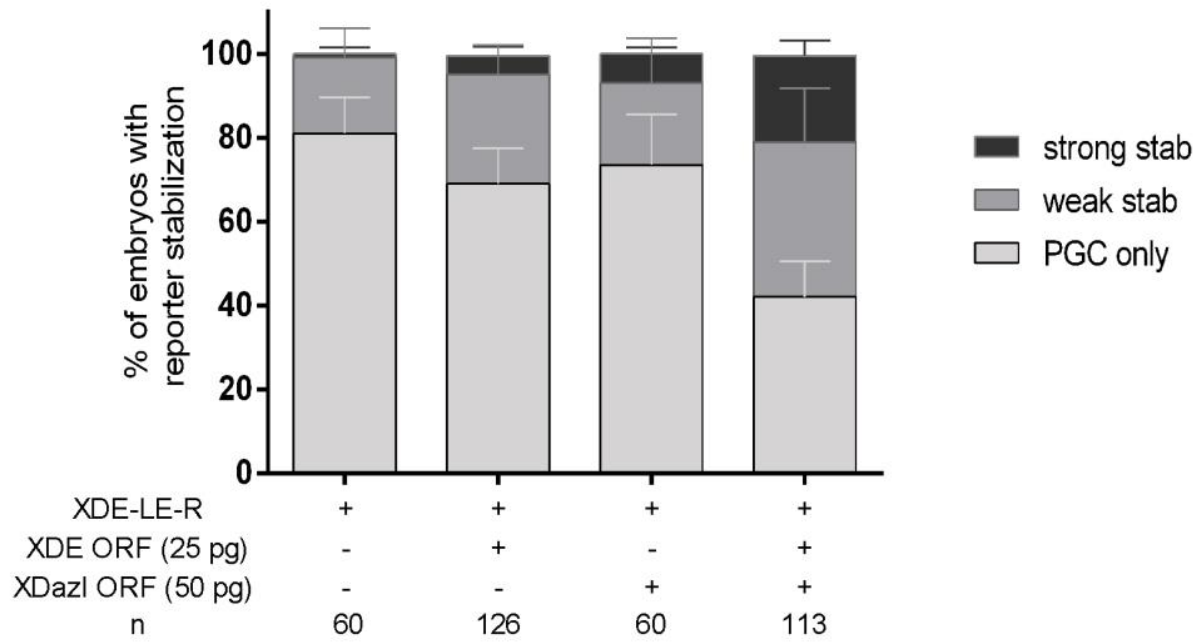

Figure 3.5. XDazl and XDE protein act synergistically in somatic XDE-LE reporter RNA stabilization.

$A+B) X$. laevis embryos were injected vegetally with either the $X D E-L E$ reporter $(500 \mathrm{pg})$ alone or with subcritical amounts of XDE ORF (25 pg) or XDazl ORF $(50 \mathrm{pg})$ or a combination of all three RNAs. Injected embryos were fixed at stage 32 and subjected to WMISH against the GFP-reporter. Whereas coinjection of the reporter RNA with one of the proteins led to no traceable or moderate somatic reporter stabilization, the coexpression of both proteins (same amounts of injected RNA) together with the reporter enhanced the stabilizing effect in a synergistic manner.

A) Experimental procedure and depiction of representative embryos.

B) Quantification of somatic reporter RNA levels (\%) scored in injected embryos. Numerical values support the observation that XDE and XDazl synergistically stabilize XDE-LE in somatic tissue. The graph summarizes the results of three independent experiments. Error bars represent standard errors. $n$, number of analyzed embryos. 
The finding that XDE and XDazl appear to cooperate in protecting $X D E-L E$ reporter RNA from somatic degradation raised the question if XDazl also cooperates with ElrB1 in stabilizing XDE-LE RNA. A synergistic function of ElrB1 and XDE protein was already described (Koebernick et al. 2010). Therefore, ElrB1 and XDazl mRNA were coinjected in subcritical amounts together with the XDE-LE reporter in Xenopus embryos (Figure 3.6 A). Only moderate effects were detectable upon overexpression of either ElrB1 or XDazl together with the $X D E-L E$ reporter RNA. If both proteins are coexpressed, we observed an additive increase in reporter stabilization in somatic cells (Figure $3.6 \mathrm{~A}+\mathrm{B}$ ). Thus, this experiment could indicate that XDazl and ElrB1 coexpression leads only to additive $X D E-L E$ reporter RNA protection from somatic clearance.

A
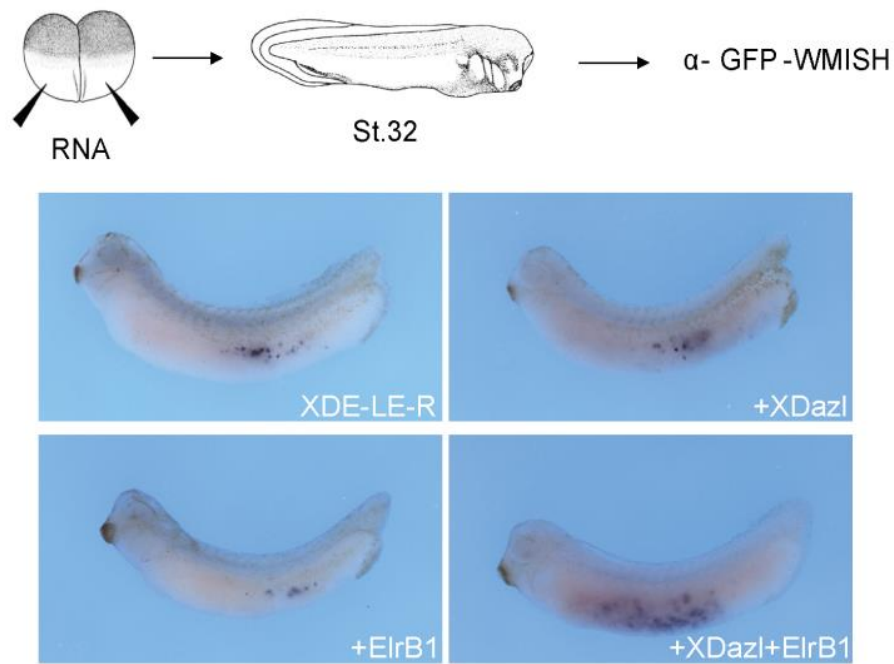

B

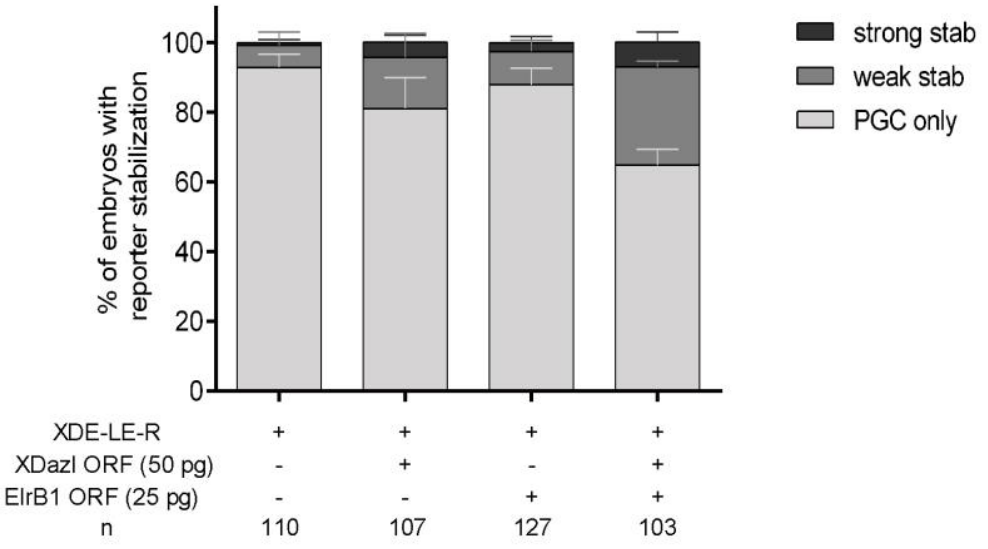

Figure 3.6. XDazl and ElrB1 protein act in an additive manner in somatic XDE-LE reporter RNA stabilization.

$\mathrm{A}+\mathrm{B}) X$. laevis embryos were injected vegetally with either the $X D E-L E$ reporter $(500 \mathrm{pg})$ alone or with subcritical amounts of XDazl ORF ( $50 \mathrm{pg}$ ) or ElrB1 ORF (25 pg) or a combination of all three mRNAs. The injected embryos were fixed at stage 32 and subjected to WMISH against the GFP-reporter.

A) Representative embryos are depicted. Whereas coinjection of the reporter RNA with one of the proteins led to no traceable or moderate somatic reporter stabilization, the coexpression of both proteins (same amounts of injected $\mathrm{mRNA}$ ) together with the reporter enhanced the stabilizing effect in an additive manner.

B) Comparison of somatic reporter RNA levels (\%) scored in injected embryos. The graph summarizes the results of three independent experiments. Error bars represent standard errors. n, number of analyzed embryos. 
ElrB1 and XDazl proteins could physically interact in the context of RNP formation on LE containing mRNAs. To address this issue, co-immunoprecipitation (ColP) experiments were carried out. RNAs coding for FLAG-epitope-tagged versions of XDazl, ElrB1 and XDE were injected vegetally into two-cell stage Xenopus embryos. Embryonic lysates were subjected to ColP analysis using antibodies against the FLAG-epitope-tag. In the fractions using either XDazI-FLAG or ElrB1-FLAG for the immunoprecipitation we found EIrA to coprecipitate with both proteins. FLAG-XDE did not coprecipitate with XDazl or Elr-type proteins (Figure 3.7). From this result we can conclude that XDazl and ElrA coprecipitate with each other.
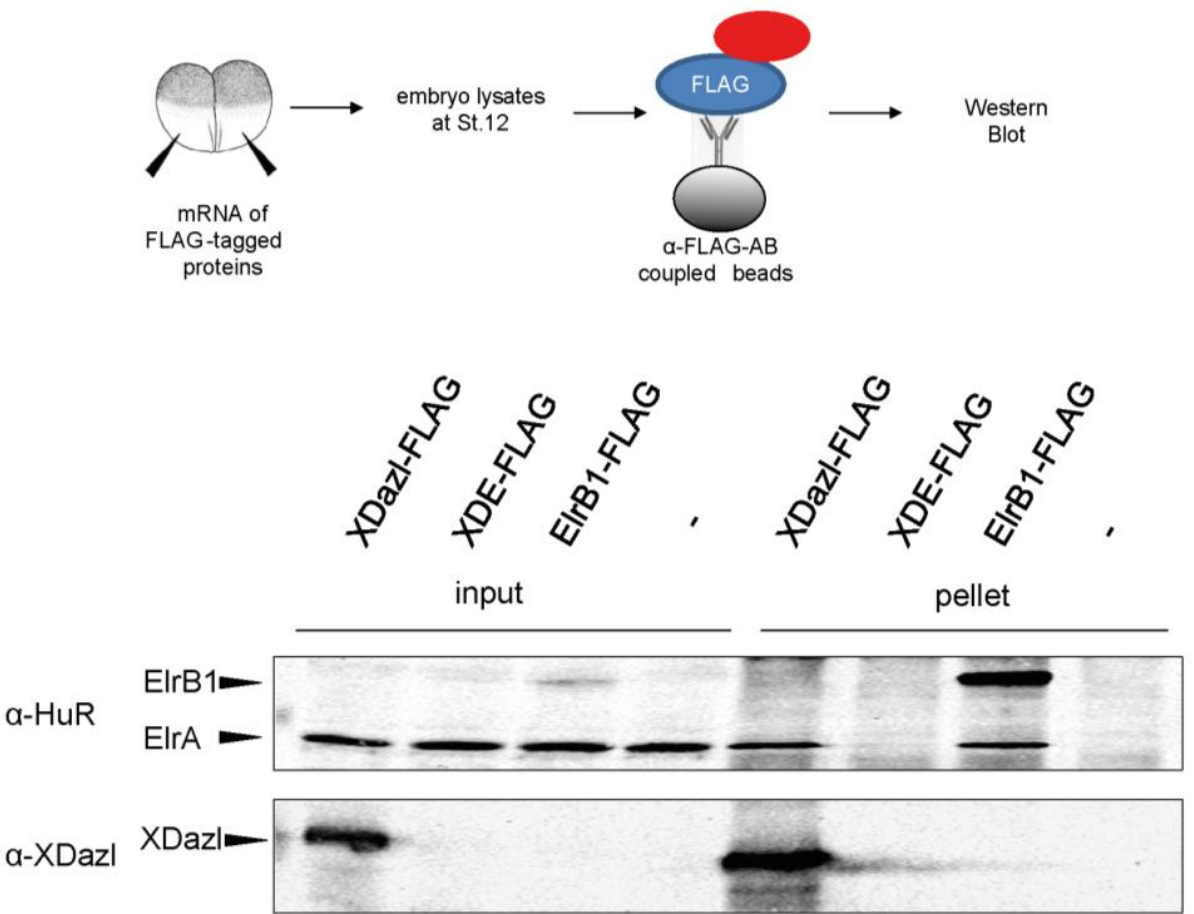

Figure 3.7. XDazl and ElrB1 coprecipitate with EIrA.

Xenopus embryos were injected vegetally into both blastomeres with FLAG-XDazl, FLAG-XDE and FLAG-ElrB1 mRNA at 2-cell stage and lysed at stage 12 for ColP using anti-FLAG antibody coupled sepharose beads. Embryo lysates, precipitated and coprecipitated proteins were analyzed in Western Blot using anti-HuR and antiXDazl antibodies, as indicated. ColP experiments showing Xenopus lysates used for ColP in the left input panels and precipitated or co-precipitated proteins in the right panels. XDazl co-precipitates with ElrA but not with endogenous ElrB1, which is present only in low amount. FLAG-ElrB1 precipitates with ElrA but not with XDazl (400 pg FLAG-XDazl, 400 pg FLAG-XDE and 400 pg FLAG-ElrB1 mRNA were injected per embryo).

Taken together, these results propose a cooperative function of XDazl and XDE and potentially ElrA/B1 proteins to protect germ cell specific mRNAs from degradation. 


\subsection{Binding of XDazl, XDE and ElrB1 proteins to the LEs of germ cell specific mRNAs}

\subsubsection{XDazl interacts directly with the XDE-LE in vitro and requires an AU-rich region surrounding the mir-18 binding site}

The experiments described above have demonstrated a protective function of XDazl in reporter stabilization experiments in vivo. Interestingly, in vitro analyses identified direct binding of recombinant XDazl protein to Cy3-labeled XDE-LE RNA (Figure $3.8 \mathrm{~A}$, Appendix Figure 6.1 A). In order to define the XDazl binding site within the XDE-LE, Cy3labeled subfragments with deletions from either the 5' (5'del1-3) or the 3' end (3'del1-7) of the LE were used in ColP experiments with XDazl protein under the same conditions as for the full length LE. As a result, the 5' region flanking and including the miR-18 binding site was found to be essential for protein-mRNA interaction (Appendix Figure 6.1 B). For quantification Electrophoretic Mobility Shift Assays (EMSAs) were performed (Figure 3.8) and the binding affinity was calculated (Appendix Table 6.1). Due to the fact that recombinant XDazl full length protein was insoluble, the RRM, known to be sufficient for RNA binding (Figure 3.4), was bacterially expressed and purified (Appendix Figure 6.3). In accordance with the ColP experiments, partial deletion of the miR-18 target site from the 5' end leads to a strong decrease in XDazl binding (5'del2), while deletion of the complete $5^{\prime}$ region $\left(5^{\prime} \mathrm{del} 3\right)$ resulted in the loss of XDazl protein binding to the XDE-LE deletion mutant (Figure 3.8 B). Deletion from the 3' end did not lead to a complete loss of binding in EMSA even if the miR target site was removed (3'del6+7) (Figure $3.8 \mathrm{C}$ ). In CoIP experiments XDazl binding was not detectable for the XDE-LE 3'del6+7 subfragments (Appendix Figure 6.1 B). Taken together, the experiments show that XDazl protein binds to the 5 ' region of the $X D E-L E$, flanking and including the miR-18 binding site. 
A
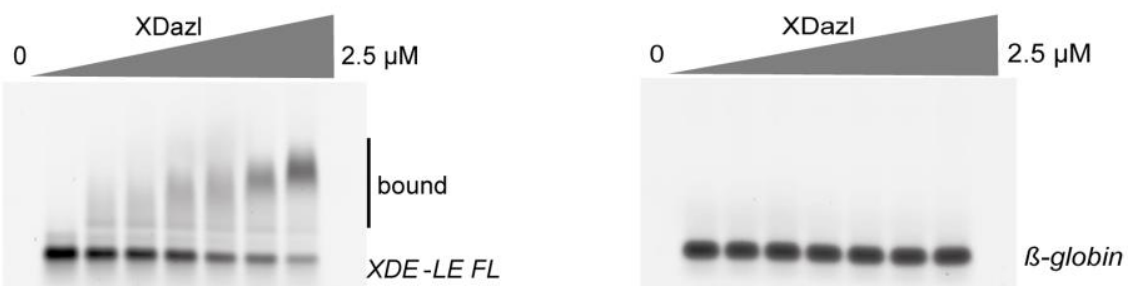

B
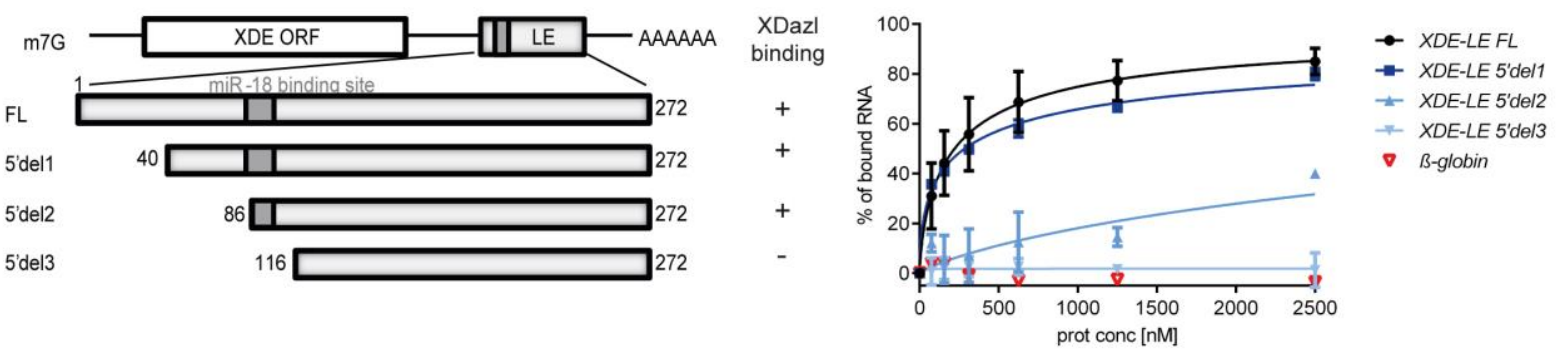

C

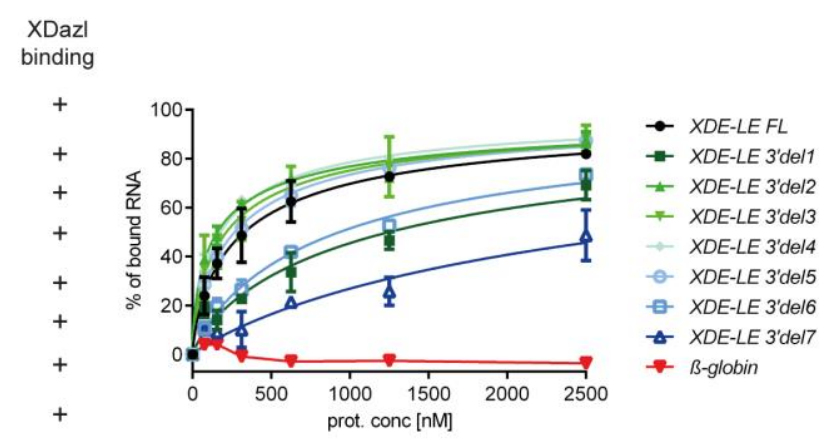

Figure 3.8. Comparative analysis of XDazl binding to subfragments of the XDE-LE.

A-C) Cy3-labeled $X D E-L E F L$ and subfragments were incubated with increasing amounts of XDazl protein $(0-$ $2500 \mathrm{nM}$ ) and analyzed for RNP complex formation by EMSA. B-globin 3' UTR served as negative control.

A) XDazl protein binds directly to the full length $X D E-L E$, whereas B-globin is not bound.

B) XDazl protein binding to the $X D E-L E$ requires the 5' regions flanking and including the miR-18 binding site. Whereas XDazl binding to the XDE-LE 5'del2 mutant is strongly impaired, the binding is lost upon incubation with $X D E-L E 5$ 'del3 mutant.

C) 3' deletion of the XDE-LE mir-18 binding site is not sufficient to prevent XDazl binding. The XDE-LE 3'del mutants are only impaired for XDazl protein binding. Even for the XDE-LE 3'del7 subfragment, in which a region 5 ' to and the miR-18 binding site is deleted, binding can be detected. 


\subsubsection{Comparison of binding efficiencies of the recombinant proteins XDazI, XDE and ElrB1 to the XDE-LE}

Arthur and colleagues found ElrB1 binding to the $X D E-L E$ and the synergism between ElrB1 and XDE in stabilizing the LE-reporter, suggesting direct binding for XDE protein to its own mRNA (Arthur et al. 2009, Koebernick et al. 2010). Thus, it was of interest to elucidate whether XDE does bind directly to the LE in its own mRNA. Therefore, bacterially expressed XDE protein was isolated (Appendix Figure 6.4) and used for EMSA experiments. These assays indeed indicate high efficiency of complex formation with the XDE-LE full length (FL) construct (Figure 3.9 A). As also XDazl and ElrB1 bind to the same LE (Figure 3.9 B-C), the question arises whether the binding site for these three proteins is the same, overlapping or separate. To elucidate this, the subfragments used above for XDazl binding assays were employed once again. The comparative diagram in Figure 3.9 D and EMSA analyses of the XDE-LE 5'del3 subfragment in Figure 3.9 A-C show that XDazl, XDE and ElrB1 have an overlapping binding site within the $X D E-L E$, including the miR-18 binding site and its 5' region. Furthermore, a $X D E-L E$ mutant (mut2b), known to impair ElrB1 binding and harboring nucleotide exchanges in the $\mathrm{A} / \mathrm{U}$-rich regions of the $X D E-L E$ (P.Arthur, unpublished variant; Appendix Figure 6.7), was tested. Comparative binding studies reveal a complete loss of binding for all three proteins to this mutant (Figure 3.9 D). These findings confirm that XDazl, XDE and ElrB1 have overlapping binding sites within the $X D E-L E$ including and flanking the miR-18 target region. 
A

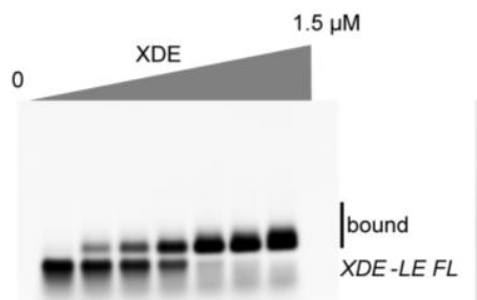

B

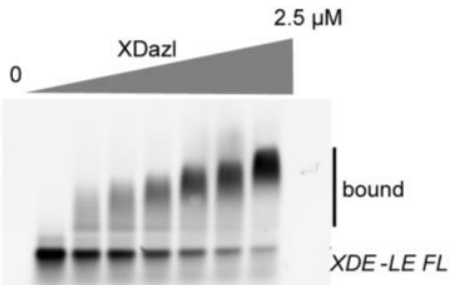

0

XDE

$1.5 \mu \mathrm{M}$
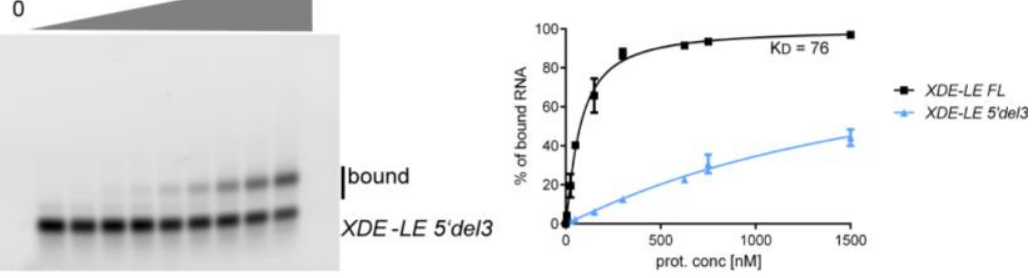

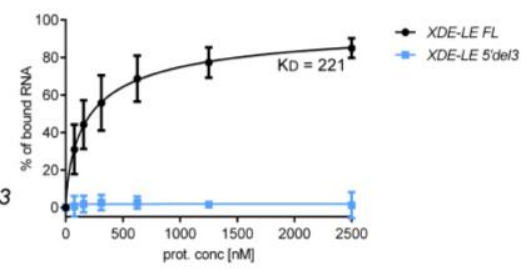

C
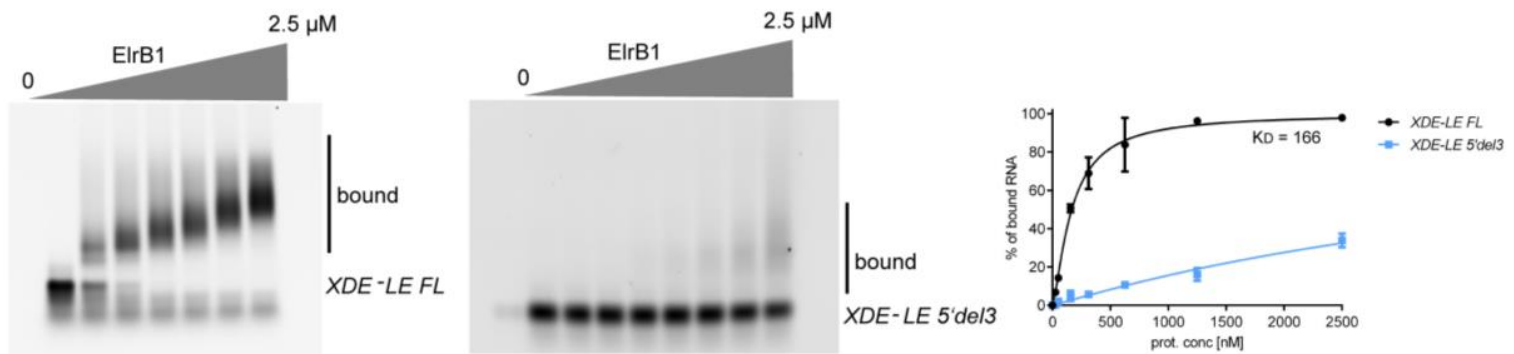

D
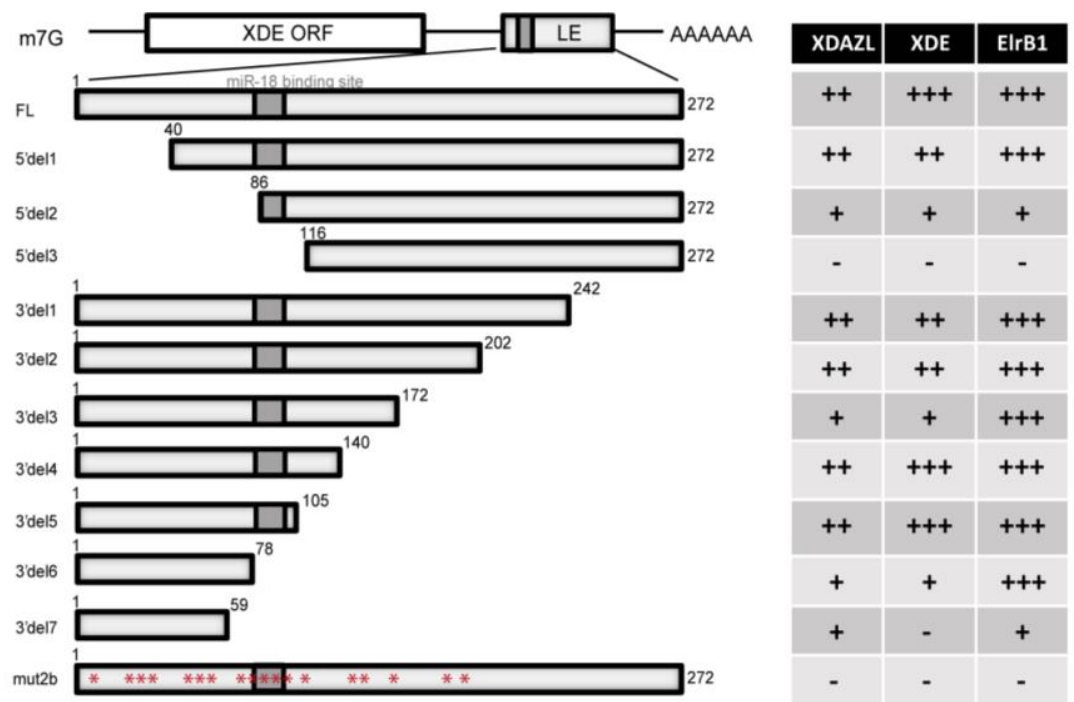

Figure 3.9. Comparison of XDE, XDazl and ElrB1 binding to the XDE-LE subfragments.

Electrophoretic mobility shift assays (EMSAs) analyzing the binding of recombinant XDE protein $(0-1.5 \mu M)(A)$, XDazl protein $(0-2.5 \mu \mathrm{M})(\mathrm{B})$ and ElrB1 protein $(0-2.5 \mu \mathrm{M})(\mathrm{C})$ to the XDE-LE full length and XDE-LE 5'del3 fragment. The RNA of the $X D E-L E$ fragments was in vitro transcribed and Cy3-labeled before the application in EMSAs.

D) Comparative diagram of analyzed XDE-LE fragments (shown on the left site) for XDazl, XDE and ElrB1 binding. For all three proteins binding to $X D E-L E 5$ 'del3 and mut2b is prevented in EMSAs. 


\subsubsection{XDazl has three independent binding sites within the XDazl-LE}

The localization element of XDazl has been mapped within its $3^{\prime}$ UTR with the help of the "REPFIND" software (Betley et al. 2002). Since NanoString nCounter multiplex analyses revealed a stabilizing effect of the XDazl protein on its own mRNA, we assayed for its direct binding to the XDazl-LE (Figure 3.10 A). CoIP and EMSA analysis showed the direct interaction of the protein to its LE (Appendix, Figure 6.2, Figure $3.10 \mathrm{~A}$ ). In order to define the XDazl binding site within the LE, subfragments of the $446 \mathrm{nt}$ long XDazl-LE full-length construct were generated (XDazl-LE 5'del1-5, 3'del1-5). The fragments were Cy3-labeled in in vitro transcription and tested in ColP (Appendix Figure 6.2) and EMSA (Figure $3.10 \mathrm{~B}+\mathrm{C}$ ) experiments using FLAG-epitope-tagged in vitro translated respectively recombinant XDazl protein. Deletions from the 5' (Figure $3.10 \mathrm{~B}$ ) as well as from the 3'end (Figure $3.10 \mathrm{C}$ ) did lead to a reduction but not the loss of XDazl binding. Even the smallest fragment XDazl-LE 5'del5 with a length of only $96 \mathrm{nt}$ was still sufficient for XDazl protein binding, but with reduced efficiency. Hence, XDazl protein appears to recognize at least two independent binding sites within the LE. Further, we wanted to analyze the ability of the XDazl protein to bind to three non-overlapping fragments namely XDazI-LE s1-s3 (XDazl-LE s1-s3, Figure 3.10 D). Hence, Cy3-labeled mRNA of these fragments and recombinant XDazl protein were applied to EMSA. We found that $X$ Dazl still binds to the XDazl-LE s1-s3 versions, however with reduced binding efficiency compared to the full length RNA of the XDazl-LE (XDazl-LE FL) (Figure 3.10 D). These experiments suggest a loss of one or more binding sites in the shorter deletion mutants (Figure 3.10 D). To monitor unspecific binding the EMSAs were performed with recombinant XDazl protein and Cy3-labeled B-globin 3' UTR (B-globin) (Figure 3.10 AD). 
A
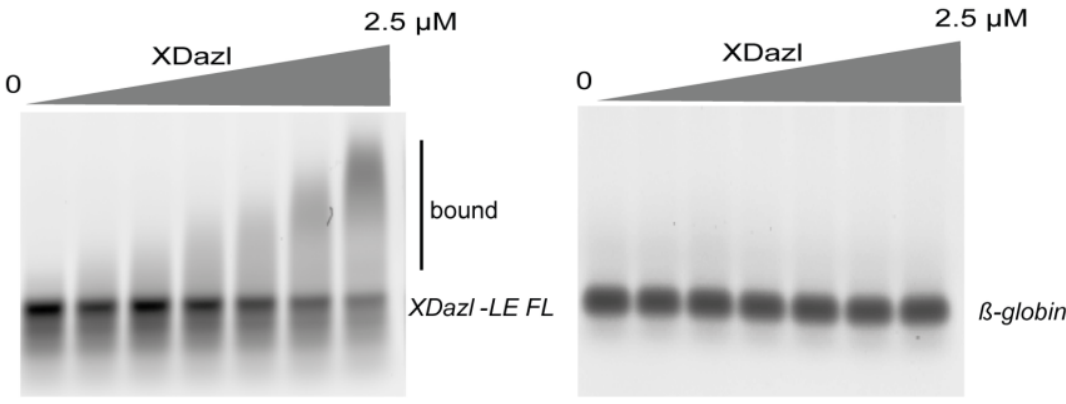

B
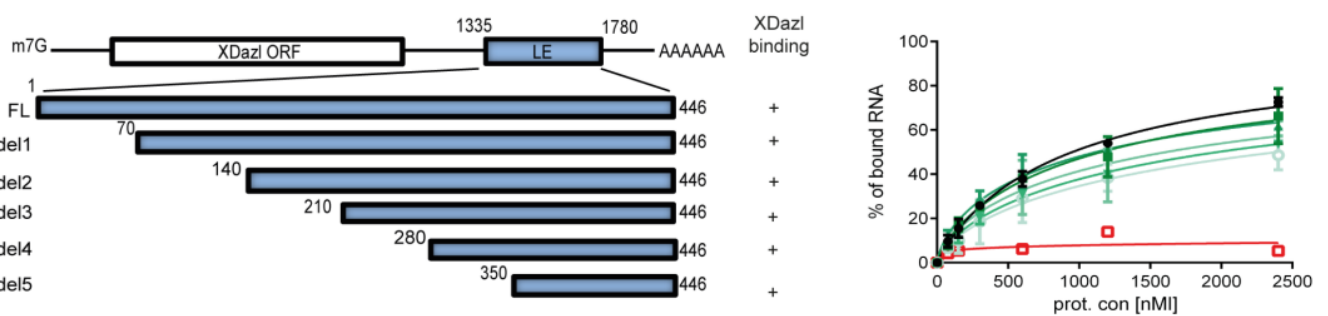

- XDAZL-LEFL

- XDAZL-LE 5'del1

- XDAZL-LE 5'del2

- XDAZL-LE 5'del3

- XDAZL-LE 5'del4

XDAZL-LE 5'de/5

- B-globin

C
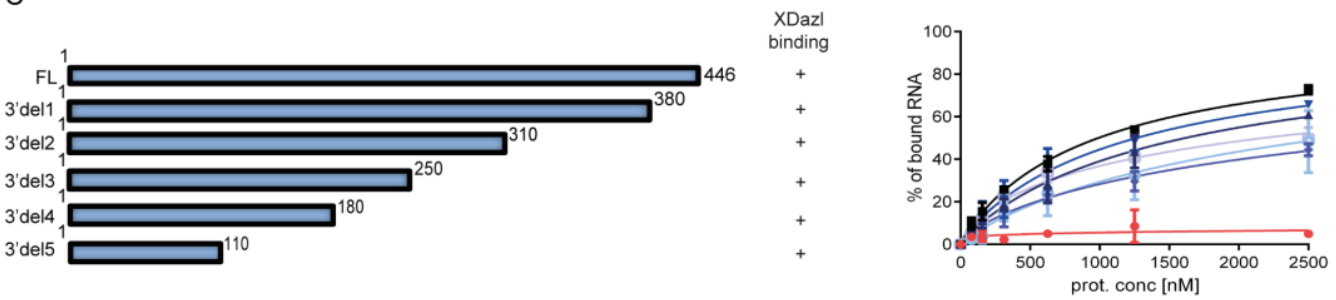

- XDAZL-LEFL

- XDAZL-LE 3'del1

- XDAZL-LE 3'del2

XDAZL-LE 3'del3

XDAZL-LE 3'del4

- XDAZL-LE 3'del5

$\rightarrow$ B-globin

D

XDazl

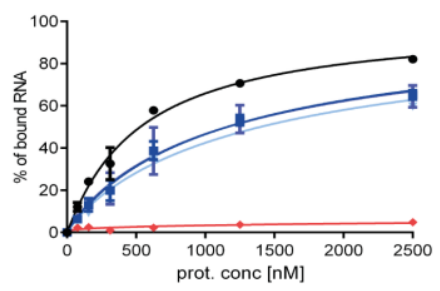

- XDazI-LEFL

- XDaltest

XDazl-LEs

XDazl-LEs2

$\rightarrow$ B-globin

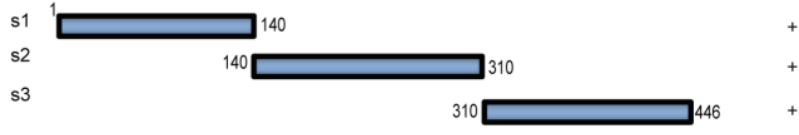

Figure 3.10. XDazl protein has three independent binding sites within the XDazl-LE.

A) XDazl protein binds to the full length $X$ Dazl-LE but not to the negative control B-globin.

$\mathrm{B}+\mathrm{C}) \mathrm{XDazl}$ protein binding to the full-length and subfragments of the XDazl-LE with deletions from the $5^{\prime}$ end (B) or the 3' end (C) was analyzed in Electrophoretic Mobility Shift Assays. Recombinant XDazl protein was incubated with Cy3-labeled XDazl-LE subfragments. For all subfragments XDazl binding is reduced.

D) EMSAs indicate reduced XDazl protein binding to the three subfragments $s 1, s 2, s 3$ of the XDazl-LE compared to the binding to the full length LE, but also shows the presence of three independent binding sites. 


\subsubsection{XDazl binds directly to the LE of multiple germ cell specific RNAs}

NanoString nCounter multiplex analysis showed that XDazl stabilizes multiple germ cell specific mRNAs (Figure 3.3). To investigate whether XDazl binds directly to the localization elements of the germ cell specific transcripts Xpat, Xcat2 and its own LE, ColP experiments with in vitro translated FLAG-epitope-tagged proteins and Cy3-labeled in vitro transcribed LEs were performed. As binding of FLAG-ElrB1 (Arthur et al. 2009) and FLAG-XDE (Löber 2008) to the XDE-LE was already described, these proteins were analyzed in parallel. As reference for the detection of unspecific RNA binding, the 3' UTR of B-globin was included in the LE-mixture (Figure 3.11 A). In Figure 3.11 A the urea gels with the separated Cy3-labeled RNAs are depicted. All Cy3-labeled RNAs could be detected in the input and the supernatant fraction. The right panel shows the pelleted RNAs indicating that all analyzed LEs of germ cell specific RNAs Xpat, XDazl, Xcat2 and $X D E$ are bound by $\mathrm{XDazl}, \mathrm{XDE}$ and ElrB1, but with different efficiencies. Whereas the binding of ElrB1 to all germline LEs is clearly detectable, the binding of XDazl protein to $X$ cat2-LE, in particular, is weak. No binding was visible for the 3' UTR of B-globin, which was used as negative control (Figure 3.11 A). To verify the binding of XDazl to the LEs of the germ cell specific RNAs Xpat and Xcat2 EMSAs with recombinant XDazl protein and Cy3-labeled RNAs were performed (Figure 3.11 B). Shift assays confirmed the binding of $X$ Dazl to the Xpat-LE, XDazI-LE and XDE-LE. Binding of XDazl to the Xcat2-LE was strongly reduced, corresponding to the results in the ColP (Figure 3.11 $\mathrm{A}+\mathrm{B}$ ). From these results we can conclude, that XDazl binds directly to the LEs of the Xpat, XDE and XDazl transcripts. 
A
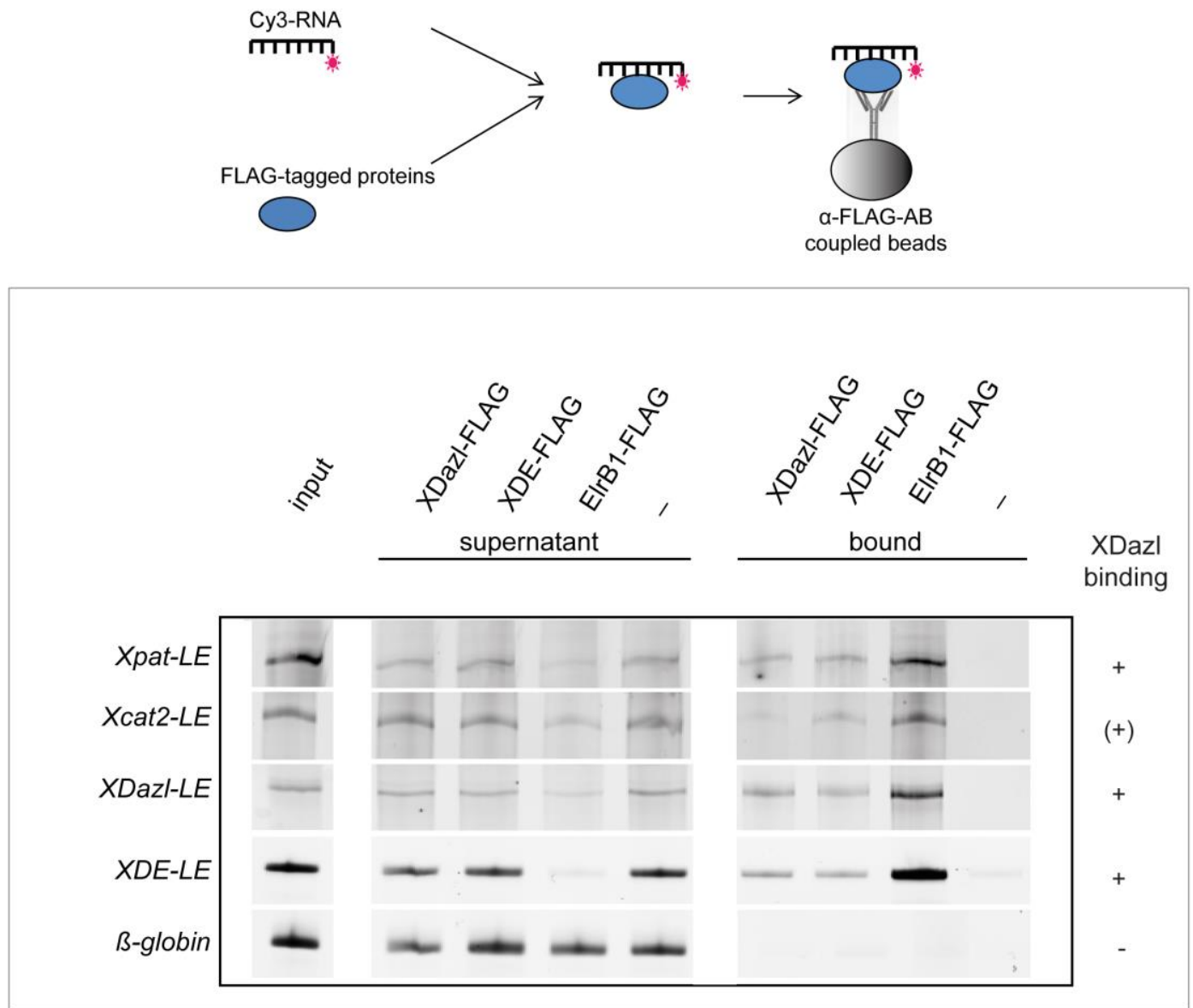

B

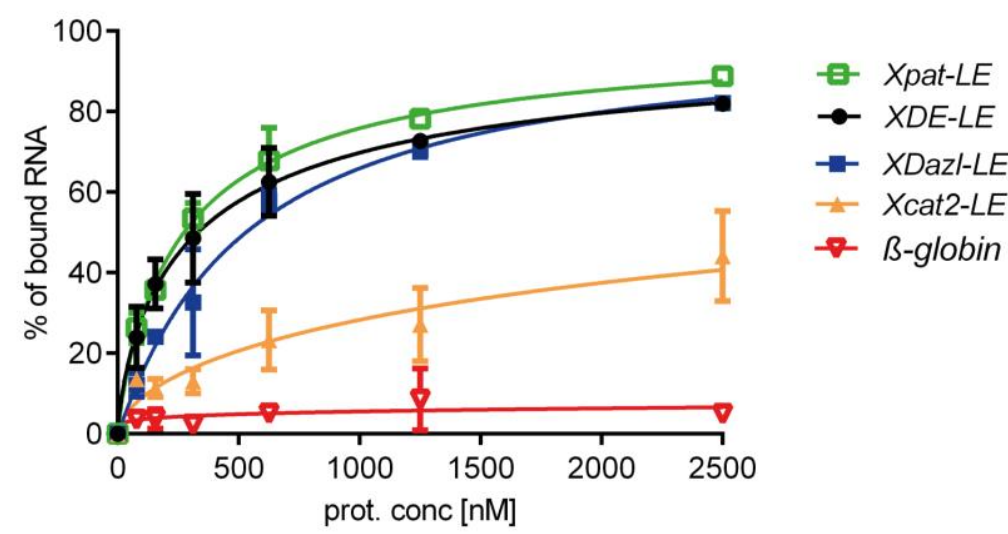

Figure 3.11. XDazl protein binds directly to the LEs of multiple germ cell specific RNAs in vitro.

A) In vitro transcribed Cy3-labeled RNA of the germline transcripts Xpat-, XDazl-, Xcat2- and XDE-LE was incubated with in vitro translated FLAG-epitope-tagged XDazl, XDE and ElrB1 proteins, immunoprecipitated, isolated and separated on urea gels. Xpat-, XDazl- and XDE-LE RNAs were bound by the three proteins. The $X$ cat2-LE is weakly bound by XDazl. B-globin 3' UTR served as negative control to monitor unspecific binding.

B) EMSA analyses using recombinant XDazl protein to compare the protein binding to the Cy3-labeled $X D E-L E$, $X$ Dazl-LE, Xcat2-LE or Xpat- LE. B-globin 3' UTR was used to monitor unspecific binding. 


\subsection{Analysis of miRNA responsibility for somatic degradation of XDazl and XDE mRNA}

Somatic degradation of germ cell specific mRNAs is thought to be effectuated by miRmediated decay. In Xenopus, the XDE-LE is targeted by miR-18 for somatic depletion (Koebernick et al. 2010). Thus, the question arises whether miRs are involved in somatic clearance of germ cell specific mRNAs in general.

\subsubsection{Germ cell specific mRNAs are degraded by miR-mediated decay}

Lund and colleagues described a siRNA approach, in which microinjected siRNAs saturate and inactivate maternal Argonaute (Ago) proteins, which are present in low amounts in Xenopus embryos but are needed for Dicer processing of pre-miRNAs at the midblastula transition (MBT). With such saturated Dicer complexes, processing of miRs is delayed in the embryo (Lund et al. 2011).

To verify whether miRs have an influence on germline transcripts, the siRNA approach mentioned above was employed. Profiling of total mRNA extracted from gastrula stage embryos was performed via NanoString nCounter multiplex analysis. Comparison between siRNA injected (100 fmol) and non-injected embryos showed an enrichment of germline specific transcripts in the first condition (Figure 3.12, Appendix 6.8.2). Cyclin B2 mRNA was analyzed as positive control as it was already shown to be stabilized under these conditions (Lund et al. 2011). The NanoString nCounter multiplex analysis revealed that Cyclin $B 2$ as well as $X D a z /$ transcripts are enriched approximately two-fold in embryos injected with siRNA. Notably, XDE mRNA was stabilized up to 7-fold suggesting that functional miRs are required for the degradation of this transcript. As expected, mRNAs which are not germ cell specific and not described to be degraded by a miR-mediated mechanism, like laminB1, are not affected (Figure 3.12). Taken together, maternally supplied germ cell specific transcripts are degraded by miRmediated decay at the onset of zygotic transcription. 

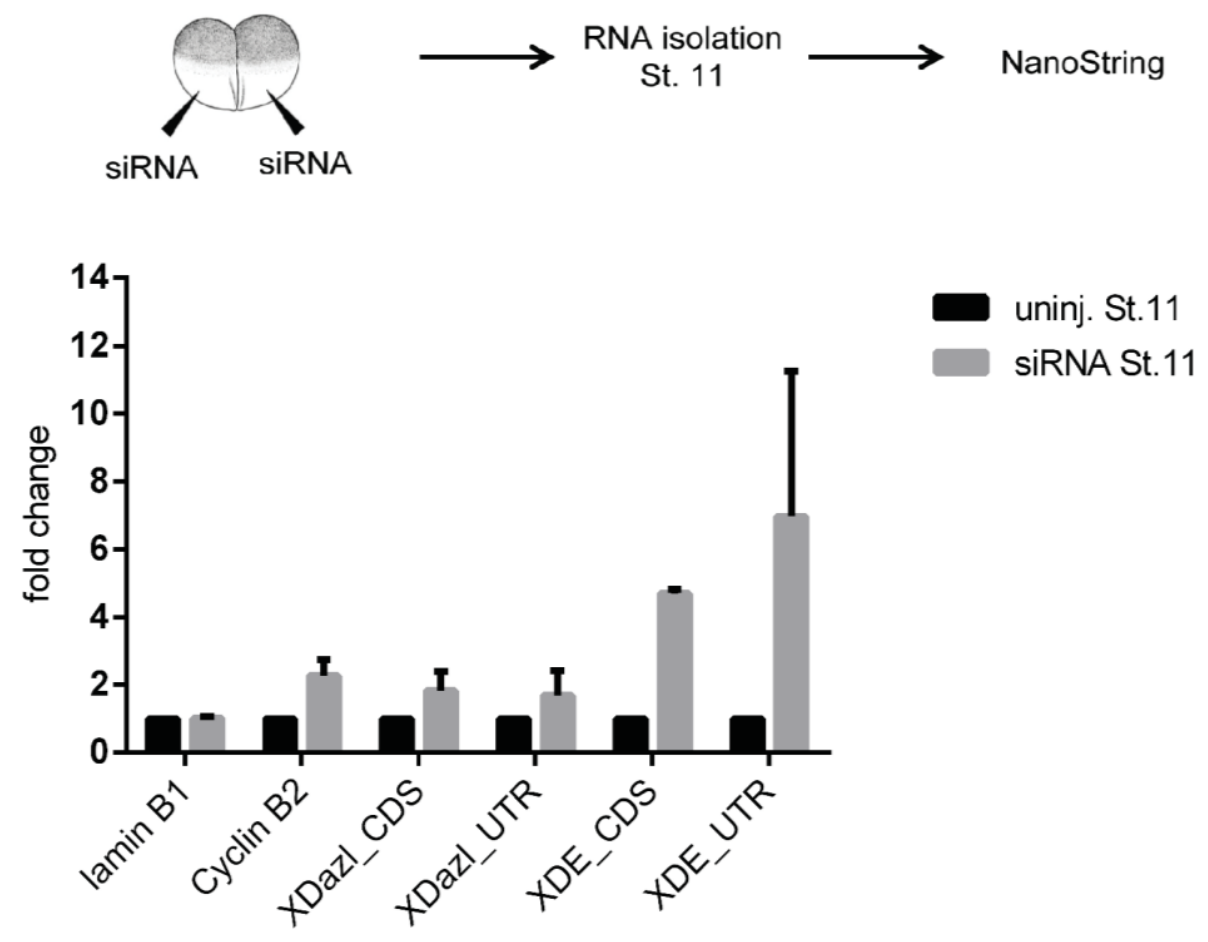

Figure 3.12. Blocking of miR processing in Xenopus embryos leads to stabilization of germ cell specific mRNAs.

Injection of excess siRNA (100 fmol) blocking the miR processing results in stabilization of germ cell specific mRNAs like XDazl or XDE, whereas mRNAs like laminB1 are not affected by the inhibition of miRNA processing. Cyclin B2 was used as positive control as it is a known target of miR mediated decay (Lund et al. 2011).

\subsubsection{Morpholino protection assay targeting the XDazl-LE s2 fragment revealed two potential miR-target sites}

Binding experiments shown in Figure 3.10 had indicated the presence of at least three binding sites for XDazl within the XDazl-LE. The original length of the XDazl-LE (446 nt) was calculated with the computer program "REFIND" and further analysis of the XDazILE is still elusive in Xenopus (Betley et al. 2002). Therefore, we asked whether a shorter fragment of the XDazl-LE is sufficient to fulfill all functions of a LE. According to the results obtained in the EMSA analysis in Figure 3.10 D, indicating three independent XDazl binding sites, reporter constructs of the XDazl-LE subfragments were generated by fusion to the GFP open reading frame. Three reporters were designed, namely XDazILE s1 (GFP ORF+140 nt), XDazl-LE s2 (GFP ORF+170 nt) and XDazl-LE s3 (GFP ORF+136 nt) (Figure 3.13 A). Microinjection of the reporters alone leads to somatic reporter degradation detectable after WMISH for XDazI-LE $s 1$ and XDazI-LE s2, whereas $X$ Dazl-LE s3 is not completely degraded in somatic tissue (Figure $3.13 \mathrm{~B}, \mathrm{C}$ ). The XDazl$L E$ s2 fragment was further analyzed for XDazl mediated somatic stabilization. Therefore, the reporter transcript was coinjected with mRNA encoding for the XDazl 
protein. Once more, the reporter was stabilized in the somatic endoderm (Figure $3.13 \mathrm{~B}$, C), recapitulating the outcome for the full-length XDazI-LE (Figure 3.2). In summary, the short XDazl-LE s2 fragment of only $170 \mathrm{nt}$ is sufficient to fulfill all functions of a bona fide localization element.

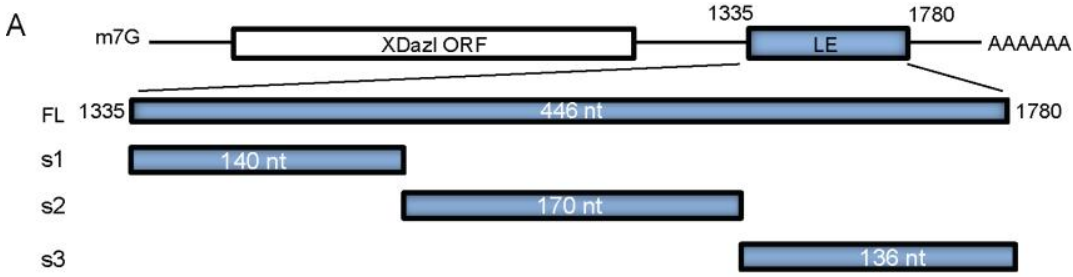

B
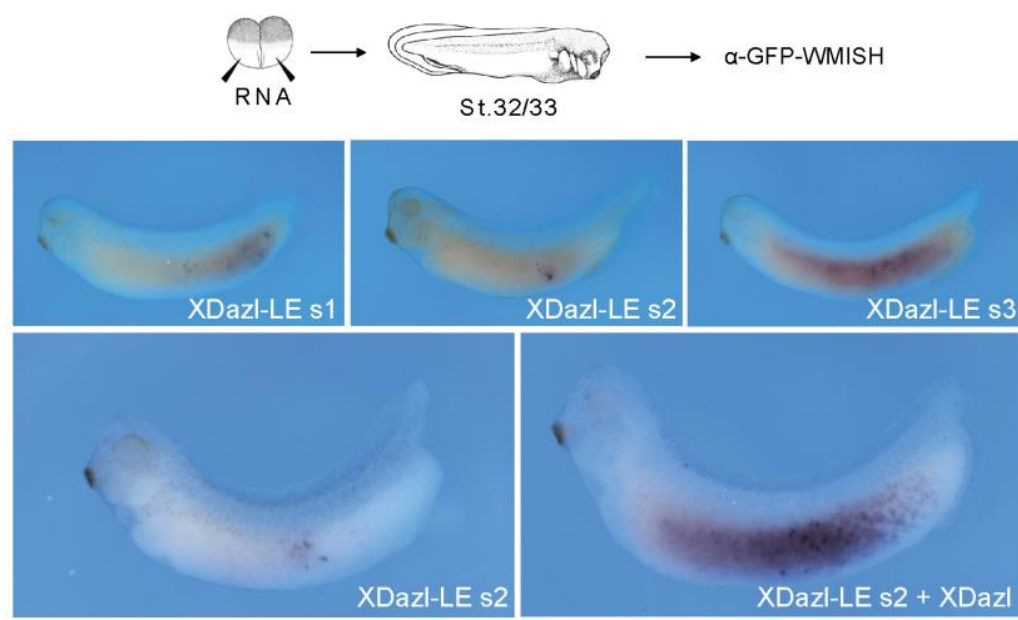

C

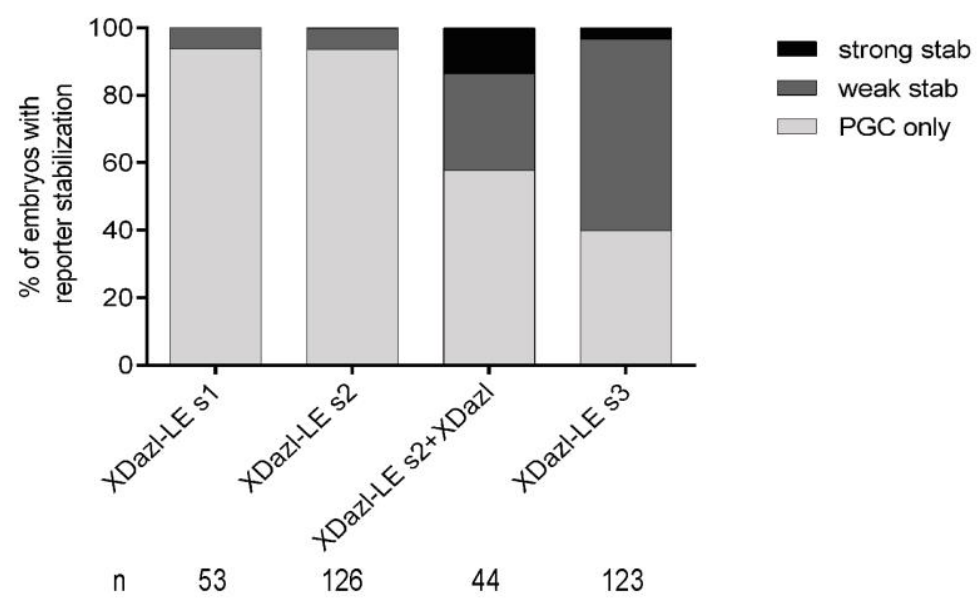

Figure 3.13. Ectopic XDazl protein expression protects the reporter RNA of the XDazl-LE s2 subfragment from somatic clearance.

A) Depiction of XDazl-LE subfragments XDazl-LE s1 (140 nt), XDazl-LE s2 (170nt), XDazl-LE s3 (136 nt),which were fused to the GFP ORF to generate reporter constructs.

$\mathrm{B}+\mathrm{C})$ Both blastomeres of two-cell stage embryos were injected vegetally with $500 \mathrm{pg}$ of XDazl-LE s1, s2 or s3 reporter RNA, the XDazl-LE s2 reporter was coinjected with $200 \mathrm{pg}$ of XDazl mRNA, embryos were cultured until stage 32, fixed and analyzed for reporter presence after WMISH against the GFP-ORF.

B) Representative embryos depicted after WMISH. Reporter injection alone resulted in restriction of the reporter to the primordial germ cells for XDazl-LE s1 and s2, whereas s3 injection led to somatic stabilization. XDazl coinjection with the XDazI-LE s2 reporter leads to somatic reporter stabilization.

C) Comparative diagram of somatic reporter RNA levels (\%) scored in injected embryos. n, number of analyzed embryos. 
The miR decay machinery mediates somatic depletion. MiRs recognize their target according to a six-nucleotide perfect matching seed sequence. In experiments above we defined a $170 \mathrm{nt}$ functional sequence of the XDazl-LE, the XDazI-LE s2 (Figure 3.13), to be sufficient to promote PGC specificity and XDazl mediated somatic stabilization. To further narrow down the region responsible for somatic degradation, morpholino (MO) protection assays were used. Binding of the MO to the miR target site in a transcript of interest blocks miR accessibility and prevents somatic decay (Koebernick et al. 2010).

To address the functional significance of different parts of XDazI-LE s2 for somatic clearance, a series of antisense morpholino oligonucleotides covering the whole s2 fragment was designed (Figure 3.14 A). XDazI-LE s2 reporter was coinjected with one morpholino at a time as previously described. Tailbud stages were subjected to WMISH against the GFP ORF. All morpholinos except MO1 interfered with somatic clearance of the reporter to a variable extent. Somatic reporter stabilization was observed in about $50 \%$ of the cases for MO2 - MO4. Notably, coinjection with MO6 and MO7 had the strongest effect (Figure 3.14 B, C). 
A

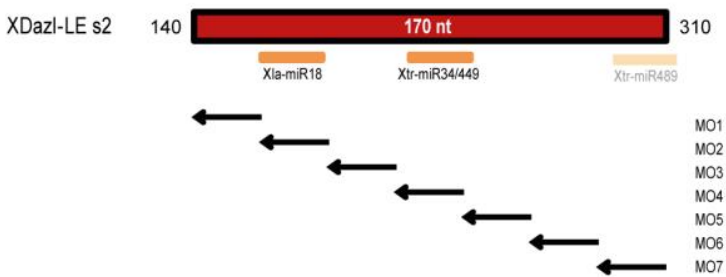

B
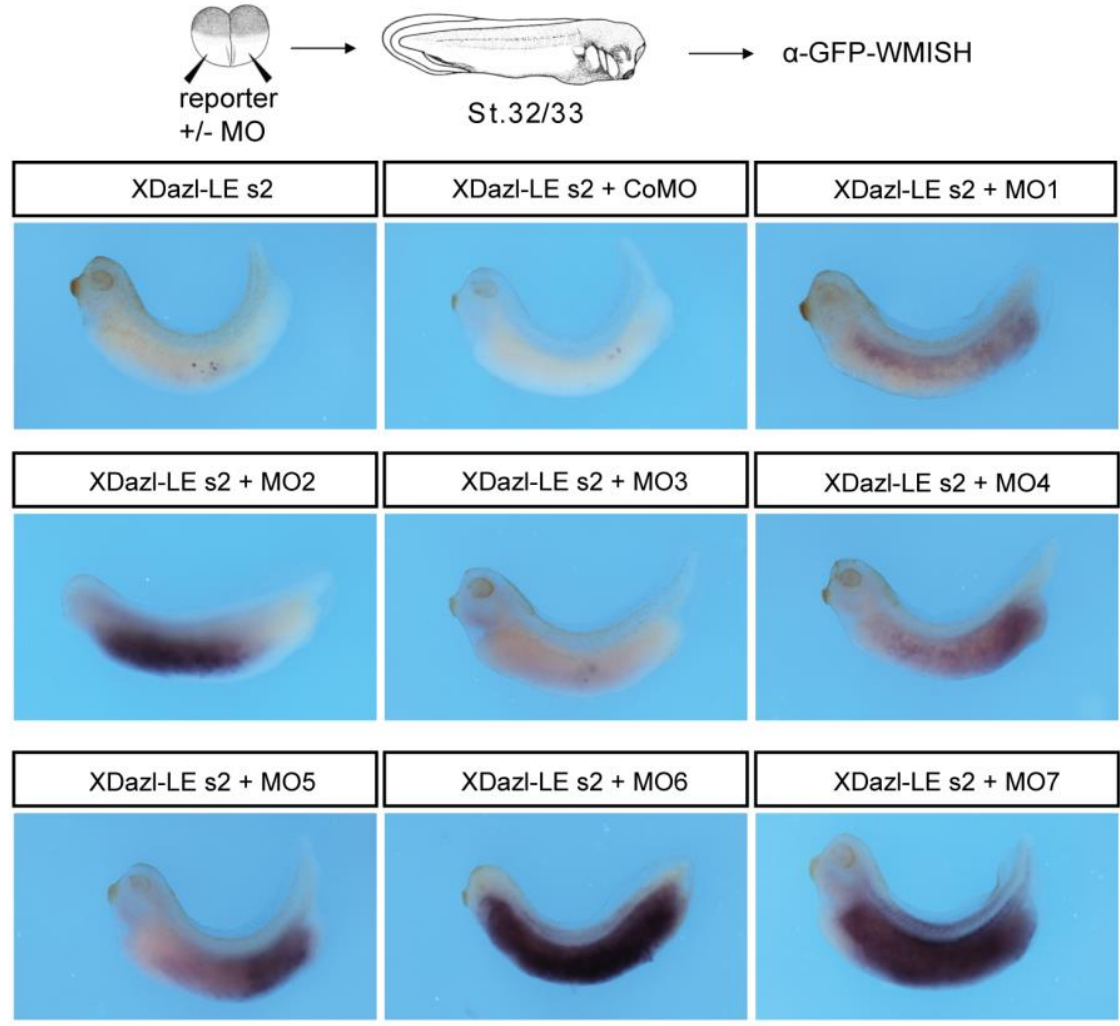

C

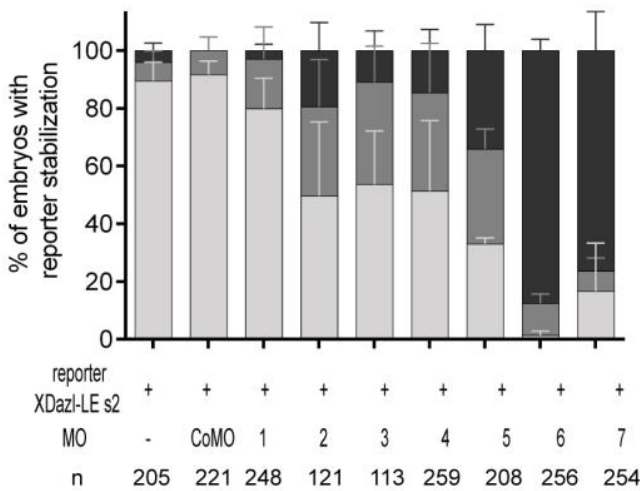

strong stab

$\square$ weak stab

$\square$ PGC only

Figure 3.14. Somatic clearance of the XDazl-LE $s 2$ fragment is strongly inhibited upon injection of target protector MO6 and MO7.

A) MO1-7 binding sites and potential miR target sites shown on the scheme of the XDazl-LE s2 reporter (170 nt). Putative miRNA binding sites (orange bars) were predicted in silico using the program miRanda. Target sites for Xla-miR-18 and Xtr-miR-34/449 were predicted. The miR-489 binding site targets only partially within the XDazl$L E$ s2 fragment, thus shown in light orange.

$\mathrm{B}+\mathrm{C})$ The XDazl-LE s2 reporter RNA $(500 \mathrm{pg})$ was injected alone or together with one of the morpholino oligonucleotides in two-cell stage embryos. The embryos were cultured until tadpole stage, fixed and subjected to WMISH against the reporter. Somatic reporter RNA clearance was inhibited to variable degrees. While moderate stabilization was detectable in embryos coinjected with MO2-MO5, the strongest effects could be achieved by MO 6 and MO7.

C) Comparison of somatic reporter RNA stabilization. Injected embryos were classified according to the strength of somatic stabilization (strong stab, weak stab, PGC only). The graph summarizes the results of three independent experiments. Error bars represent the standard errors. n, number of analyzed embryos. 
The region targeted by MO6 and MO7 potentially contains miR target sequences. Those miR target sites can be predicted using bioinformatics tools. All known mature $X$. laevis and $X$. tropicalis miR sequences [miRbase, Xenbase-miR catalog, 2014] were screened for complementarity with the XDazl-LE FL using the miRanda program (Enright et al. 2003, Betel et al. 2008, Betel et al. 2010). Four different miRs were predicted to bind to the XDazl-LE s2 fragment (Figure 3.15). Notably, Xenopus laevis miR-18, respectively Xenopus tropicalis miR18a/b, known to be responsible for somatic XDE-LE reporter degradation (Koebernick et al. 2010), was among the predicted miRs. The region covered by $\mathrm{MO} 2$ corresponds to the miR-18a/b binding site (Figure $3.14 \mathrm{~A}$ ). Another predicted binding site, for miR-34/449, was fully blocked by MO4 and partially by MO5 binding (Figure $3.14 \mathrm{~A}$ ). In the sequence covered by MO6 and MO7 one known $X$. tropicalis miR target site was predicted, namely for miR-489 (Figure 3.14 A). Bioinformatics showed that three nucleotides of the miR-489 seed sequence map outside of the XDazl-LE s2 reporter fragment. Therefore, further unknown miRs might be involved in somatic XDazl-LE s2 degradation. Nevertheless, testing the involvement of the miR-18, miR-34/449 and miR-489 on somatic XDazl-LE degradation is of interest and needs be addressed in further analysis.

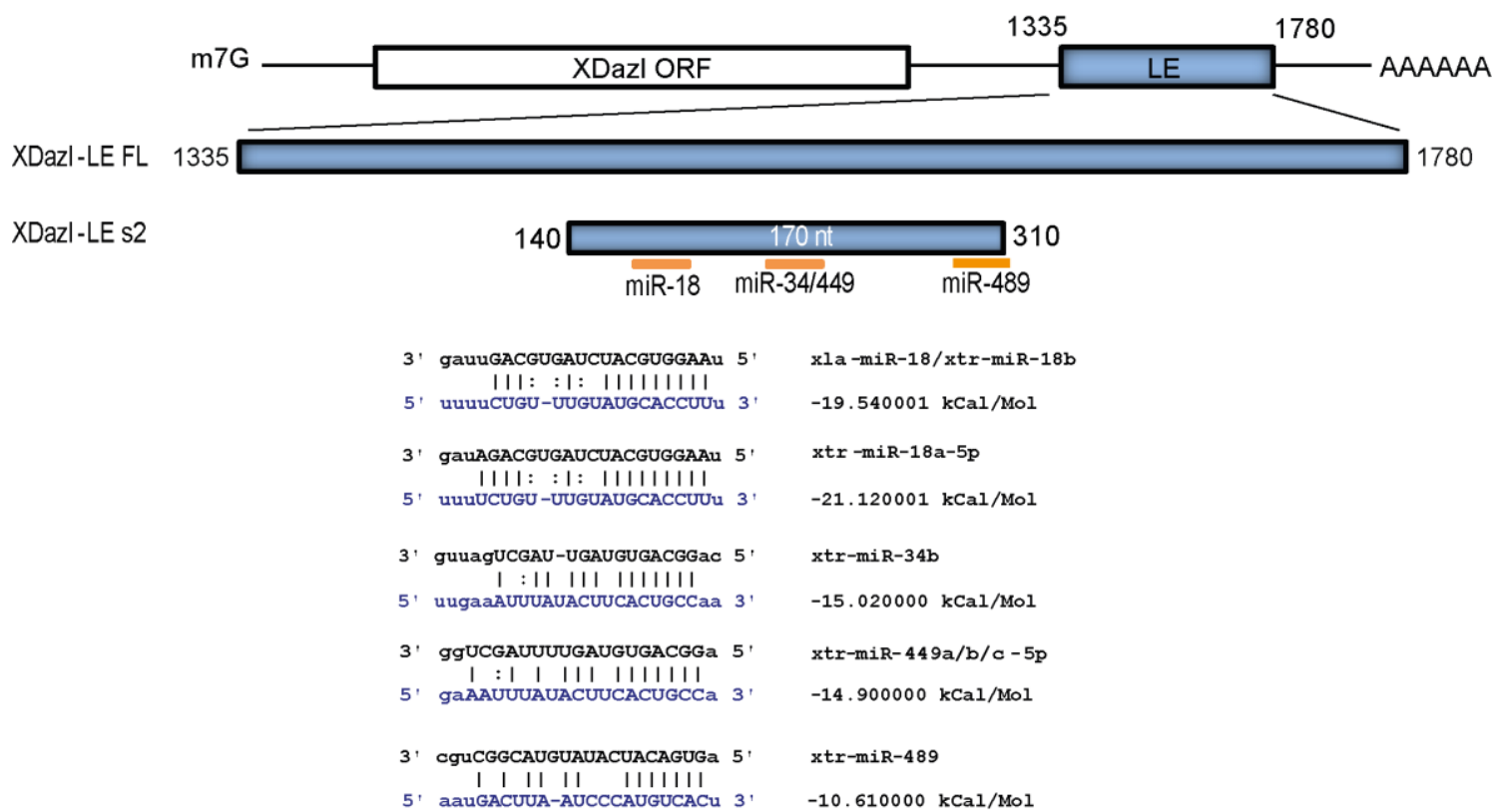

Figure 3.15. Multiple miR binding sites can be predicted in the XDazI-LE s2 subfragment.

In silico analysis predicted four different miR binding sites within the XDazI-LE s2 subfragment. Predicted target sites for xla-miR18 (xtr-miR18a/b), xtr-miR-34, xtr-miR-449 and xtr-miR-489 are shown within the XDazl-LE s2 fragment. 
In this study we have described the ability of XDazl to protect germ cell specific mRNAs from degradation. To establish the relationship between XDazl protein binding and germ cell-specific suppression of mRNA clearance, we took advantage of the same set of MOs described above (Figure 3.14 A). The Cy3-labeled RNA of XDazI-LE s2 was subjected to EMSAs with recombinant XDazl in the presence of one of these morpholinos. We found that the affinity of the XDazl protein to the XDazI-LE s2 is reduced in general compared to that of XDazl and XDazI-LE FL also in absence of a morpholino (Figure 3.16, Figure 3.10). Unexpectedly, XDazl protein binds the XDazI-LE s2 with higher efficiency in the presence of the CoMO and MO7. However, the addition of the morpholino 1-6 led to reduced binding between XDazl protein and XDazI-LE s2 with the most dramatic effect upon coincubation with $\mathrm{MO} 2$, which covers the miR-18 target site (Figure 3.16, Figure 3.14 A). The strong somatic XDazl-LE s2 reporter stabilization mediated by MO6 and MO7 can not be explained with loss of XDazl binding (Figure 3.16). Taken together, these results reveal an important XDazl binding site within the XDazl-LE s2 fragment overlapping with the miR-18 binding site (Figure 3.14, Figure 3.16).

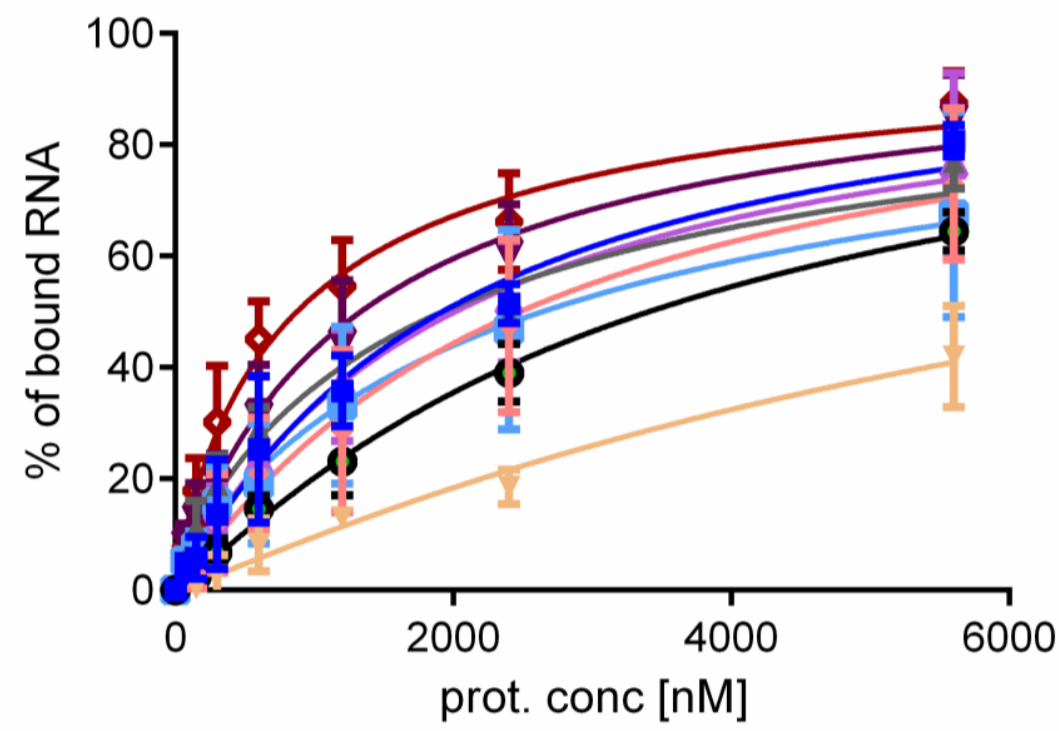

$\rightarrow-s 2$

$\leftarrow s 2+\mathrm{MO} 1$

$\because \quad s 2+\mathrm{MO} 2$

$\rightarrow \quad s 2+\mathrm{MO} 3$

- $s 2+\mathrm{MO} 4$

$-s 2+\mathrm{MO5}$

$-s 2+\mathrm{MO6}$

$\rightarrow s 2+\mathrm{MO} 7$

$\multimap s 2+$ CoMo

prot. conc $[\mathrm{nM}]$

Figure 3.16. Blocking the miR-18 binding site within the XDazl-LE s2 fragment interferes with XDazl protein binding.

Electrophoretic mobility shift assays display reduced XDazl protein binding to the XDazl-LE s2 fragment in the presence of MO2 compared to the binding without morpholino or with MO1,3-7. The coincubation of the XDazI-LE s2 fragment with XDazl in presence of MO7 and CoMO increases binding affinity. 


\subsection{Blocking of zygotic XDazl translation does not affect RNA stability}

Maternal depletion experiments identified XDazl protein as important component for germ cell specification. In the absence of XDazl, PGCs are impaired in their ability to migrate from the ventral to the dorsal endoderm and disappear until stage 40-43 (Houston and King 2000a). Further analysis describing XDazl functions on the molecular level have been performed in reporter assays but not on the level of endogenous mRNAs (Maegawa et al. 2002, Wiszniak et al. 2011).

We could show above that ectopic XDazl expression has a stabilizing effect on endogenous germ cell specific mRNAs. Thus, we asked whether the knock down of XDazl leads to decreased levels of the same mRNAs. We first analyzed expression of both, XDazl protein and mRNA. We collected Xenopus oocytes and embryos of different developmental stages (Figure 3.17 A) and applied them to Western Blot analysis (Figure $3.17 \mathrm{~B}$ ) and qRT-PCR (Figure $3.17 \mathrm{C}$ ). We saw the presence of the $37 \mathrm{kDa}$ XDazl protein from early oocyte stages right up to embryonic stage 13/14 in accordance with the results from Mita and colleagues (Mita and Yamashita 2000). In the mature egg, a second band can be detected at $40 \mathrm{kDa}$. As XDazl contains the consensus sequence of the MAP kinase recognition motif (PXS/TP) and since MAP kinase is activated during Xenopus oocyte maturation (Gotoh et al. 1995, Yoshida et al. 1995), this modification is most probably caused by MAP kinase-mediated phosphorylation. Between stage 15 to stage 41 only weak XDazl protein bands are detectable. By stage 43 a strong XDazl signal at $\sim 34 \mathrm{kDa}$ can be detected (Figure 3.17 B). XDazl mRNA expression was determined using quantitative RT-PCR. Corresponding to the data from the Western Blot analysis this expression revealed a strong maternal supply of XDazl mRNA during early embryonic stages that dramatically decreases after MBT (Figure $3.17 \mathrm{C}$ ). These data indicate that XDazI transcripts and protein are maternally supplied but rapidly decreased after MBT. Strong zygotic XDazl protein translation is not detected before tadpole stage, when PGCs migrate actively towards the future gonads. 
A

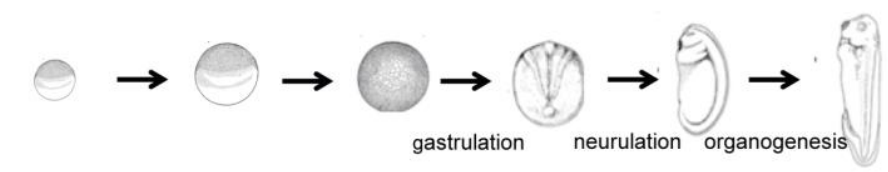

B
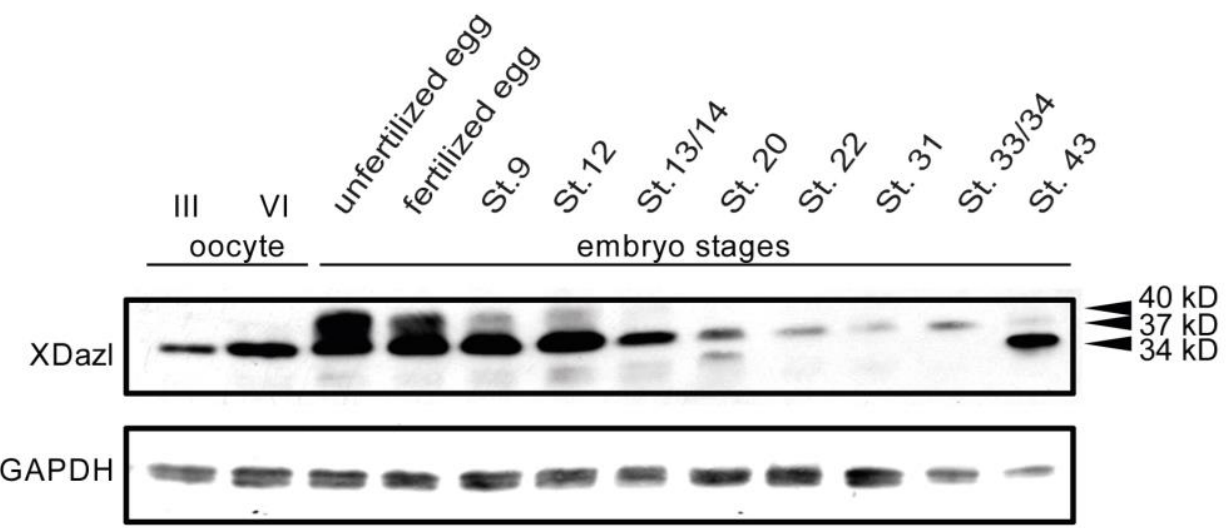

C

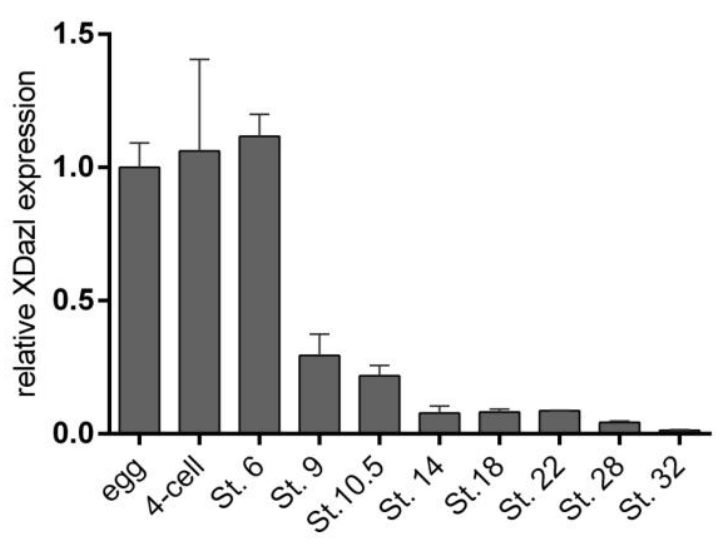

Figure 3.17. XDazl protein and mRNA expression from oogenesis to embryogenesis.

A) Schematic view of some of the embryonic stages analyzed in Western Blot in B)

B) Western Blot against XDazl showing the protein presence from oogenesis to the end of gastrulation (St.13/14). After stage 20 and before stage 43 XDazl is nearly not detectable but the protein expression starts again at stage 41/43, when PGCs migrate towards the future gonad. GAPDH was used as control.

C) Quantitative RT-PCR analysis of XDaz/ mRNA normalized to ODC at different stages of embryogenesis.

In general zygotic transcription starts at mid blastula transition (MBT) according to Xenopus stage 8,5. In this period also miRs are transcribed by the zygote (Svoboda and Flemr 2010). However, temporal expression of germ cell specific mRNAs is still unknown, as PGCs are still transcriptionally silenced after MBT. Furthermore, the abundant maternal supply of germ cell specific transcripts and proteins makes it difficult to determine the onset of zygotic expression. To address this issue, we fertilized Xenopus laevis eggs with sperm from Xenopus tropicalis (Figure $3.18 \mathrm{~A}$ ). Although $X$. tropicalis has a diploid set of chromosomes and $X$. laevis exhibits a pseudo-tetraploid genome both organisms are closely related and viable hybrid offspring could be generated. Notably, the late stage hybrid embryos developed strong phenotypic 
abnormalities at stage 49 with edemas, enlarged heart anlage and incorrect folding of the intestine (Figure $3.18 \mathrm{~B}$ ). Since the $X$. laevis and the $X$. tropicalis XDazl sequences differ in specific regions, the zygotic (paternal) transcription onset can be determined in RTPCR. As control $X$. laevis eggs were fertilized with $X$. laevis sperm. The embryos were cultured and fixed at different stages of embryogenesis. Total RNA from these embryos was isolated and analyzed by RT-PCR for the start of paternal transcription of $X$ DeadSouth, $X D E$ and $X D a z l$ mRNA. Primers for this experiment were designed in a way that either the $X$. laevis or the $X$. tropicalis transcript is amplified. This experiment revealed that the zygotic transcription of XDeadSouth, XDE and XDazl mRNA starts at stage 17 (Figure $3.18 \mathrm{C}$ ). At stage 17, a band for XDazl transcripts can be detected in RT-PCR, at stage 26 the expression was increased (Figure $3.18 \mathrm{C}$ ). These results reveal that zygotic $X D a z l$ transcription begins later than the general transcription in germ cells, which starts at stage 14 (Venkatarama et al. 2010). 
A

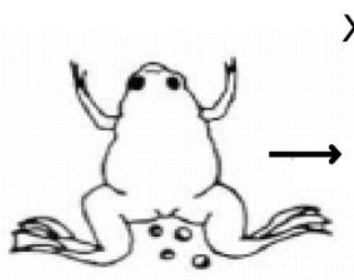

X.tropicalis sperm

$\mathrm{X}$. laevis eggs

B

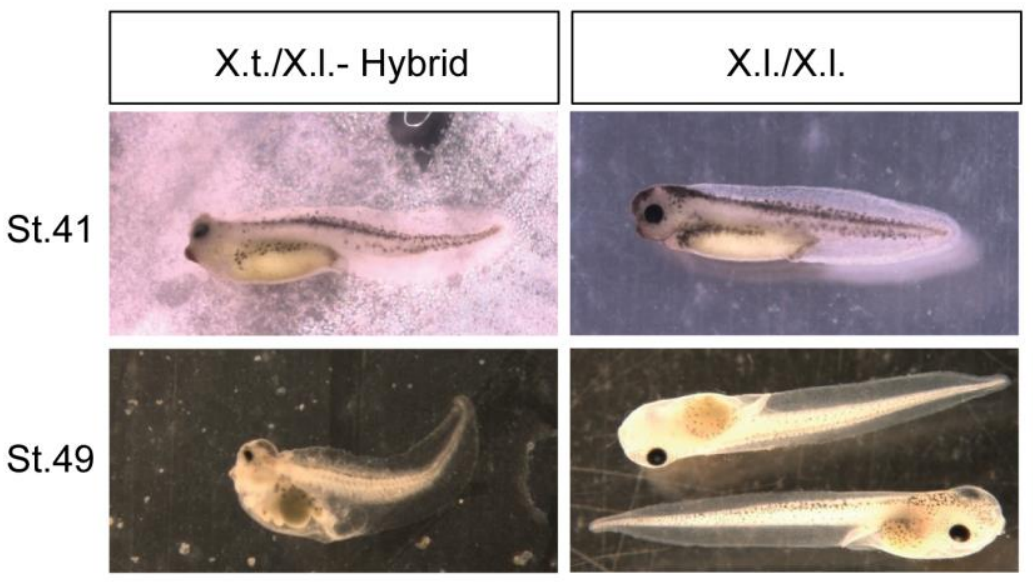

C

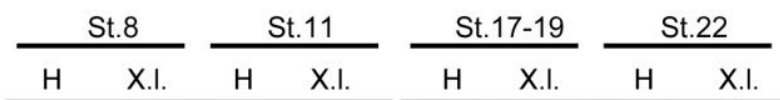

XDeadSouth X.t.

XDeadSouth X.I.

XDE X.t.

XDE X.I

ODC

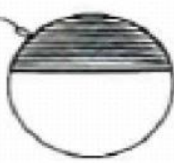

$\longrightarrow \quad$ RNA isolation

modified from N. Mise, 1994

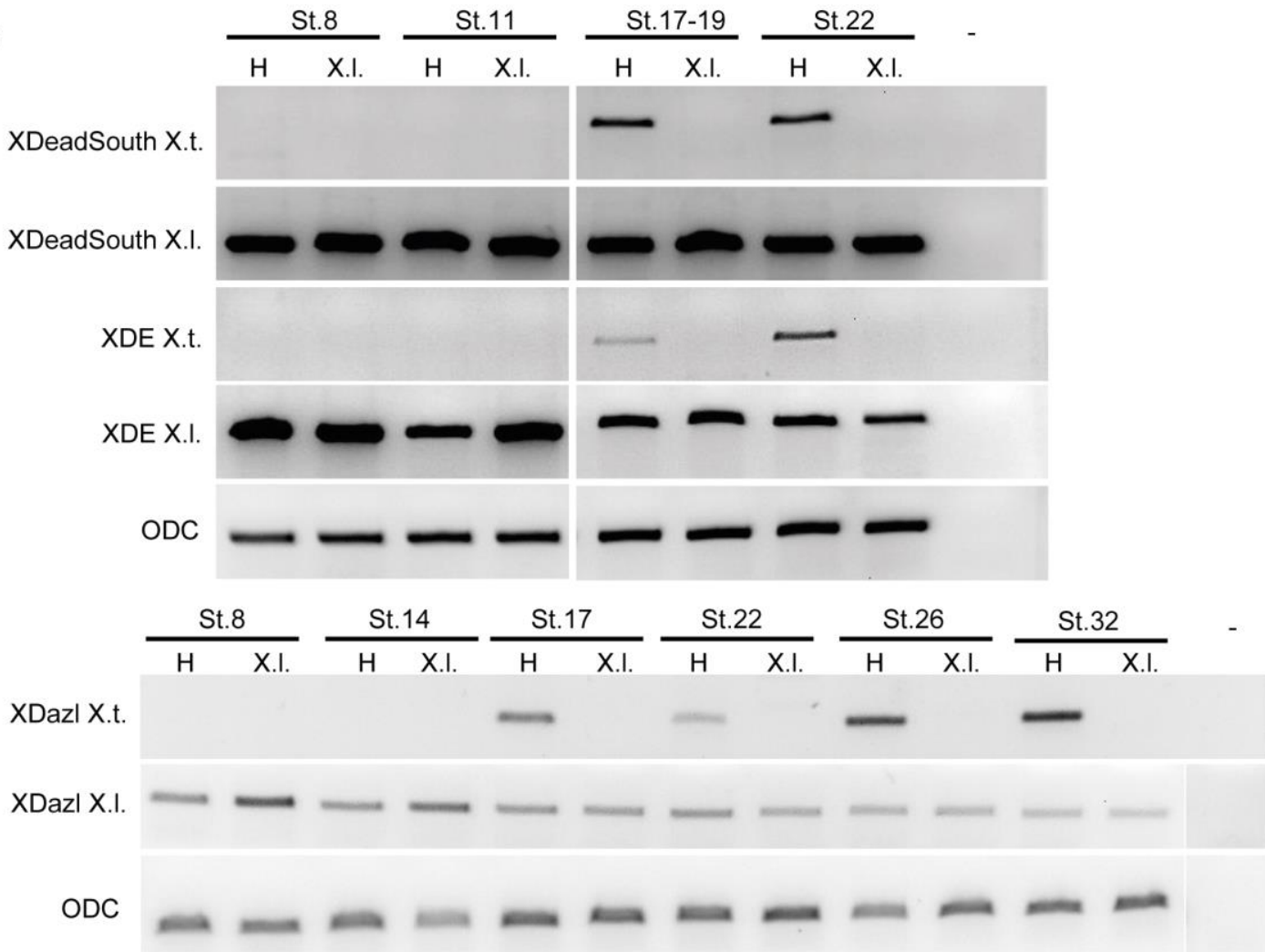

Figure 3.18. XDazl transcription starts at embryonic stage 17.

A) Scheme of the experimental procedure showing the $X$. laevis female laying eggs, which were fertilized with $X$. tropicalis sperm to generate hybrid $(\mathrm{H})$ embryos for RNA isolation at different developmental stages. As control $X$. laevis eggs were fertilized with $X$. laevis sperm and these embryos were treated in parallel with the hybrid embryos.

B) $X$. tropicalis $X$. laevis hybrids show strong developmental defects in the late stages of embryogenesis compared to the control embryos (X.I/X.I).).

C) RT-PCR analyzing the paternal $(\mathrm{H})$ transcription start of the germ cell specific XDeadSouth, $X D E$ and $X D a z l$ mRNA compared to the expression of the X. laevis (X.I.) transcripts. 
Different than in the experiment from Houston, who depleted maternal XDazI mRNA with Phosphothioate-Oligonucleotides followed by host transfer (Houston and King 2000a) to achieve XDazl knock down, we used morpholino oligonucleotides to block the translation of the XDazl protein in the embryo. To analyze the functionality of the morpholino, in vitro transcription coupled translation of a plasmid containing the morpholino binding site and the XDazl ORF in presence or absence of the XDazl morpholino (XDazl MO) was carried out (Figure 3.19 A). To monitor unspecific effects caused by the morpholino we additionally used a control morpholino (CoMO) with a random sequence. Protein expression was monitored by Western Blot using an antibody against XDazl protein. The presence of the XDazl morpholino but not that of the control morpholino led to complete loss of XDazl protein expression (Figure 3.19 A). To generate a loss of function situation in Xenopus embryos the XDazl morpholino was injected vegetally into both blastomeres of a two-cell stage embryo. Protein lysates of the injected embryos at different stages were generated and subjected to Western Blot analysis using the XDazl antibody. Additionally, we analyzed the expression of XStaufen protein as control. XStaufen expression was not affected by the XDazl morpholino oligonucleotide (Figure 3.19 B). At stage five of embryogenesis we detected high levels of XDazl protein, also in the presence of the XDazl morpholino, which is most probably due to the strong maternal protein supply (Figure $3.19 \mathrm{~B}$ ). However, XDazl protein levels were slightly reduced at stage eight upon XDazl MO injection. This effect was more pronounced in protein lysates of stage 13 Xenopus embryos (Figure $3.19 \mathrm{~B}$ ). These data indicate that the injected $\mathrm{XDazl} \mathrm{MO}$ can efficiently block translation of XDazl in vivo and in vitro. 
A

\begin{tabular}{ccccccc} 
XDazl & + & + & + & + & + & + \\
XDazl- & 0,1 & 1 & 8 & - & - & - \\
MO & $\mu \mathrm{g}$ & $\mu \mathrm{g}$ & $\mu \mathrm{g}$ & & & \\
$\mathrm{CoMO}$ & - & - & - & $0,1 \mu \mathrm{g}$ & $1 \mu \mathrm{g}$ & - \\
\cline { 2 - 6 } & & & & & & \\
a-XDazl & & & & & &
\end{tabular}

B
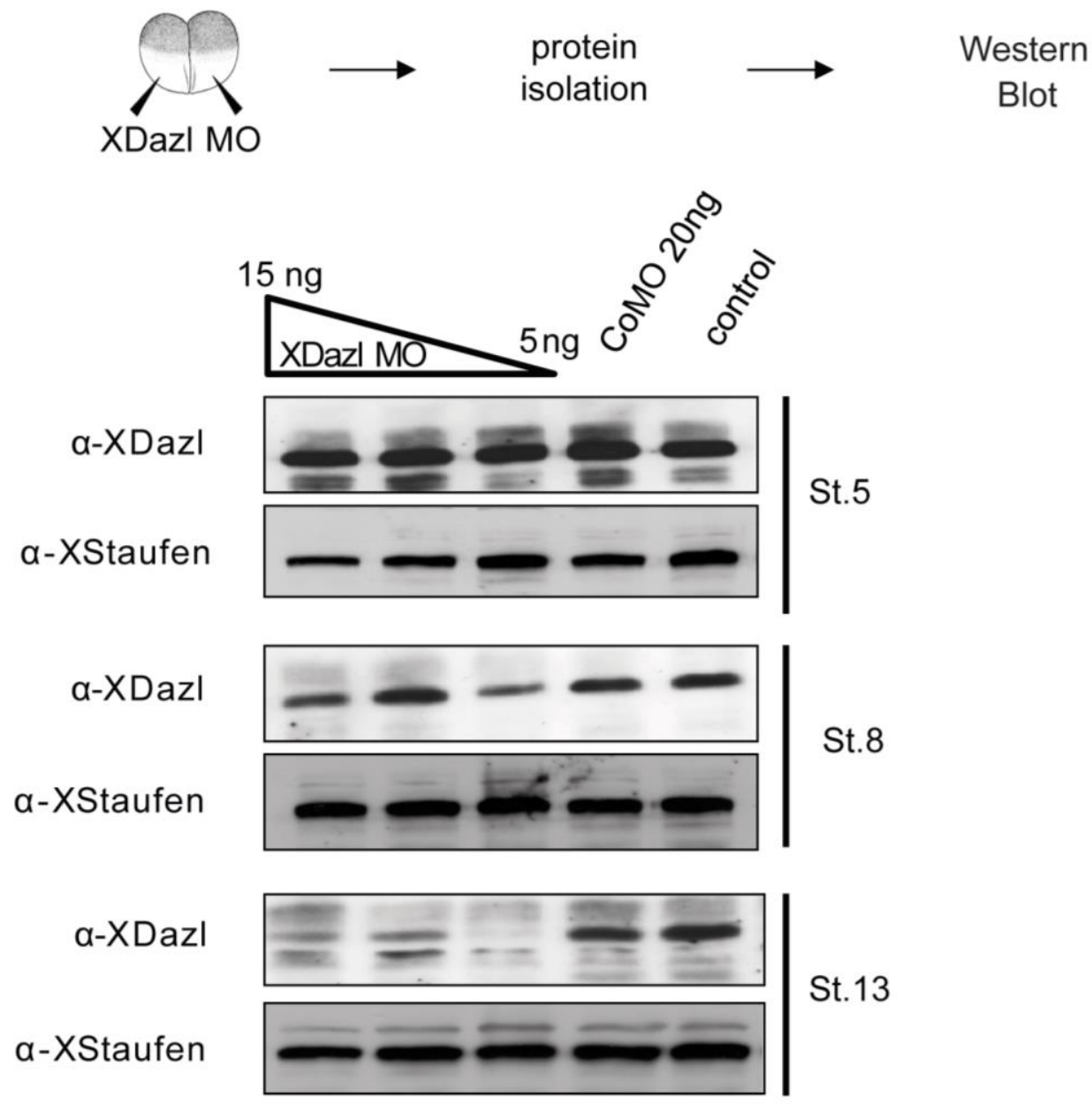

Figure 3.19. XDazl morpholino blocks the translation of the XDazl protein efficiently in vitro and in vivo.

A) Western Blot against XDazl protein after in vitro transcription coupled translation of XDazl plasmids +/coincubation with the XDazl MO or CoMO showed strong translational inhibition upon XDazl morpholino presence.

B) Western Blot analysis determining XDazl expression in embryos injected with XDazl morpholino (XDazl MO), control morpholino (CoMO) or uninjected embryos at different developmental stages. At stage 13 XDazl protein expression is reduced in XDazl morpholino injected embryos, whereas no reduction was detectable in control morpholino or uninjected embryos. XStaufen was used as control. 
In order to analyze if suppression of XDazl function leads to reduced germ cell specific mRNA stability, XDazl-MO injected stage 32 embryos were subjected to WMISH against the germ cell specific mRNA Xpat and Xpat positive cells (PGCs) were counted (Figure 3.20 A+B). The XDazl morpholino injection resulted in a decrease in the number of Xpat positive cells at stage 32 (Figure $3.20 \mathrm{~B}$ ). Nevertheless, also the injection of the CoMO led to a significant albeit weaker reduction of PGC number (Figure $3.20 \mathrm{~B}$ ). This observation suggests that the injection procedure into the germ plasm region may already interfere with germ cell formation. As shown previously, the knockdown of XDE leads to a reduced number of PGCs as well (Horvay et al. 2006). Thus, we hypothesized that knockdown of both, XDazl and XDE, would result in a more dramatic effect than knockdown of XDazl alone. Therefore, we coinjected XDE and XDazl morpholino and analyzed the embryos after WMISH against Xpat. However, coinjected embryos display equivalent numbers of PGCs as in the XDazl or XDE only knockdown situation (Figure $3.20 \mathrm{C}$ ). In order to analyze, whether the knock down of XDE and XDazl leads to reduced mRNA stability after the onset of zygotic transcription, we analyzed mRNA levels of XDazl and Xpat by qRT-PCR in XDE and XDazl morpholino injected embryos at stage 14. However, not even the knockdown of both, XDE and XDazl, resulted in a significant reduction of XDazl or Xpat mRNA levels (Figure $3.20 \mathrm{D}$, E). From these experiments we can conclude that the knockdown of XDazl in the embryo leads to reduced germ cell number. Even so, a destabilizing effect on the germ cell specific mRNA Xpat could not be detected in embryos after MBT. 
A
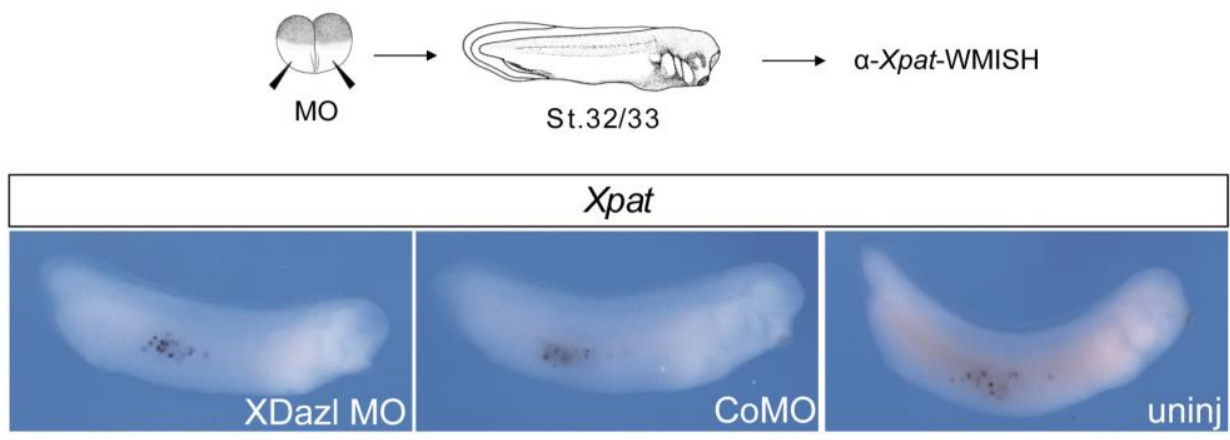

B

C
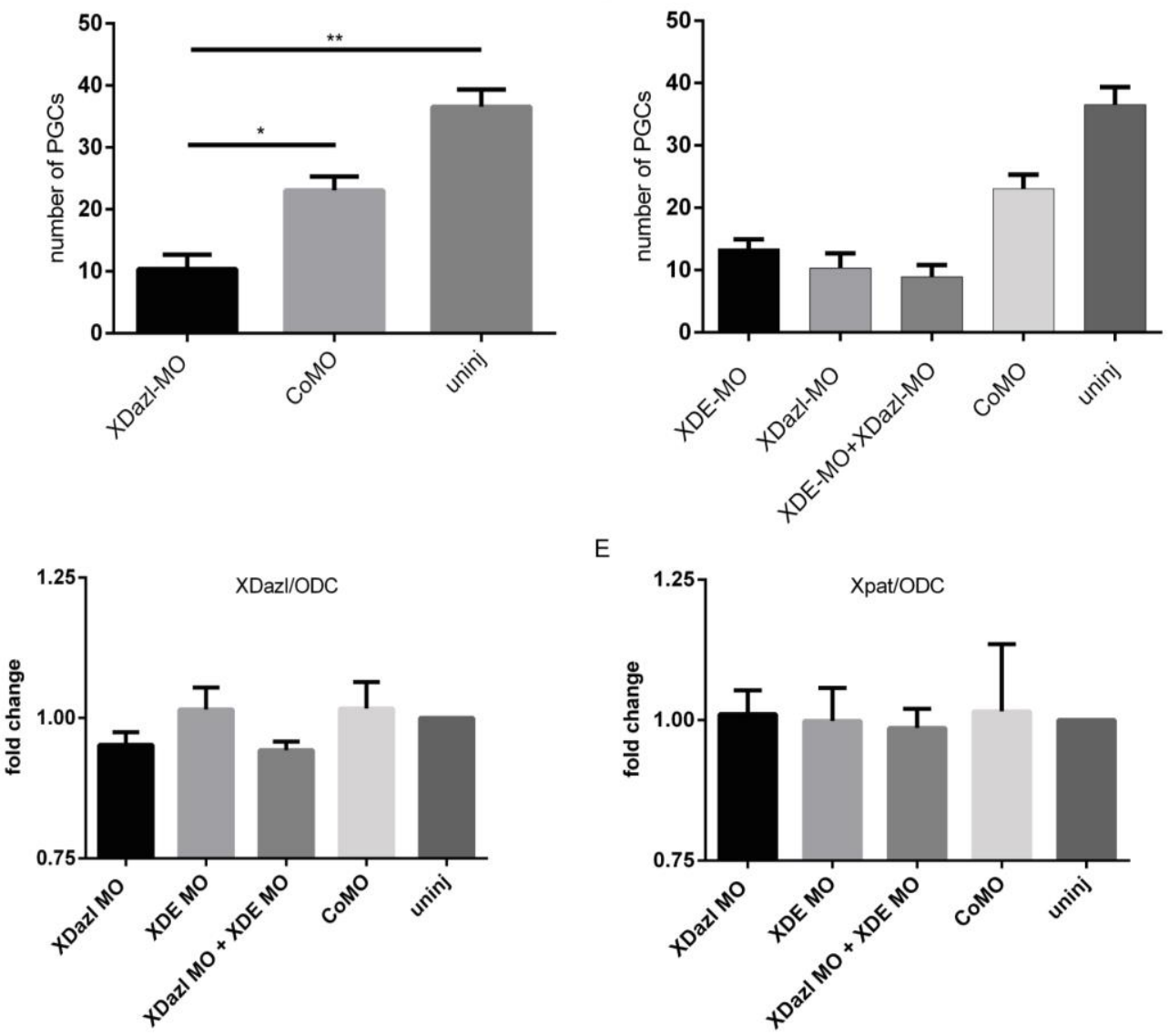

E
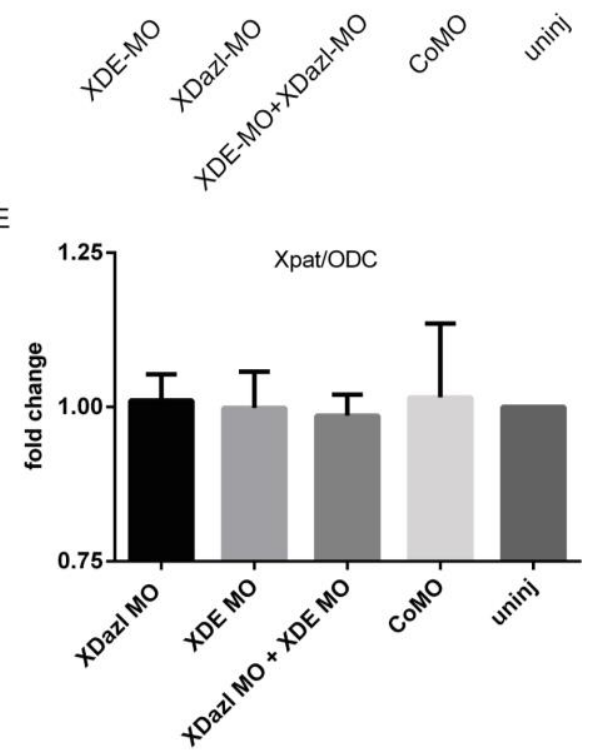

Figure 3.20. XDazl morpholino injection results in a reduced PGC number in embryos.

$\mathrm{A}+\mathrm{B})$ Two-cell stage embryos were injected with $15 \mathrm{ng}$ of XDazl or $30 \mathrm{ng}$ of control morpholino in the vegetal hemisphere and cultured until stage 32/33, fixed and in situ hybridization against Xpat mRNA was performed.

A) Representative embryos are depicted showing the Xpat expression in PGCs. In all three shown examples PGCs are detectable.

B) Morpholino injected embryos were analyzed after $\alpha$-Xpat-WMISH and clearing for their PGC number. XDazl morpholino injected embryos were compared with control morpholino injected or uninjected embryos for their PGC number. Graph summarizing the PGC number of XDazl morpholino (15 ng), control morpholino (30 ng) injected or uninjected embryos from three independent experiments + standard error. For each column 75 embryos ( 25 embryos per experiment) were evaluated. * $p$ values in an unpaired t-test $<0.05$,

${ }^{* *} p$ values in an unpaired t-test $<0.005$.

C) Comparative diagram for the PGC number (Xpat positive cells) in XDE and XDazl (15 ng each) morpholino coinjected embryos next to embryos injected with either XDE (15 ng), XDazl (15 ng) or control morpholino (30 $\mathrm{ng})$.

$\mathrm{D}+\mathrm{E})$ Quantitative RT-PCR analysis of endogenous XDazl (D) and Xpat (E) mRNA levels normalized to ornithine decarboxylase (ODC) mRNA levels. XDazl and Xpat mRNA quantities are displayed in relation to the mean values from stage 14 uninjected embryos, which were set to 1 . Total mRNA was extracted in two independent experiments from 3 snap-frozen embryos (single embryo mRNA analysis) at the stage 14 . No significant decrease of endogenous XDazl or Xpat mRNA levels could be detected. Error bars represent the standard deviation. 


\subsection{Colocalization of XDazl protein with germline specific mRNAs at the vegetal cortex in Xenopus oocytes}

\subsubsection{XDazl colocalizes with XDE-LE and XDazl-LE at the vegetal cortex of Xenopus oocytes}

In the Xenopus oocyte, it has been shown that proteins with important functions in vegetal mRNA localization, such as Vg1RBP (Zhang et al. 1999), VgRBP60 (Lewis et al. 2004), ElrA and ElrB1 (Arthur et al. 2009), XStaufen (Yoon and Mowry 2004) and 40LoVe (Czaplinski et al. 2005) are enriched at the vegetal cortex resembling the distribution of their target mRNAs. As the $X D E$ and $X D a z l$ mRNAs localize to the vegetal pole in Xenopus oocytes, a potential earlier function of XDazl protein during vegetal transport or anchoring of germ cell specific mRNAs seems possible (Houston and King 2000a, Horvay et al. 2006).

For this issue, we injected Cy3-labeled XDE-LE or XDazl-LE mRNA into the nucleus of Xenopus stage III oocytes, fixed them after 3 days of incubation and performed immunostaining against endogenous XDazl protein (Figure 3.21 A). Enrichment of XDazl was visible in transport particle-like structures in the vegetal hemisphere, which may correspond to mRNA transport particles and at the vegetal cortex (Figure $3.21 \mathrm{~B}, \mathrm{C}$ ). In these regions the XDazl protein also colocalizes with the XDE-LE and XDazI-LE RNA.

These results correspond to the idea of a possible early function for the XDazl protein in the transport pathway or during anchoring of target mRNAs at the vegetal cortex in Xenopus oocytes. 
A

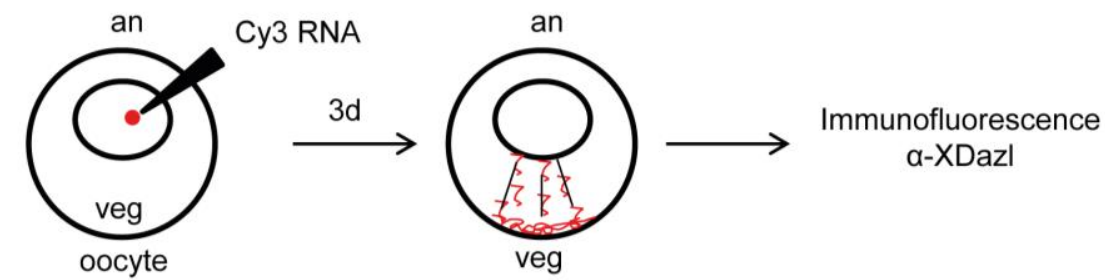

B
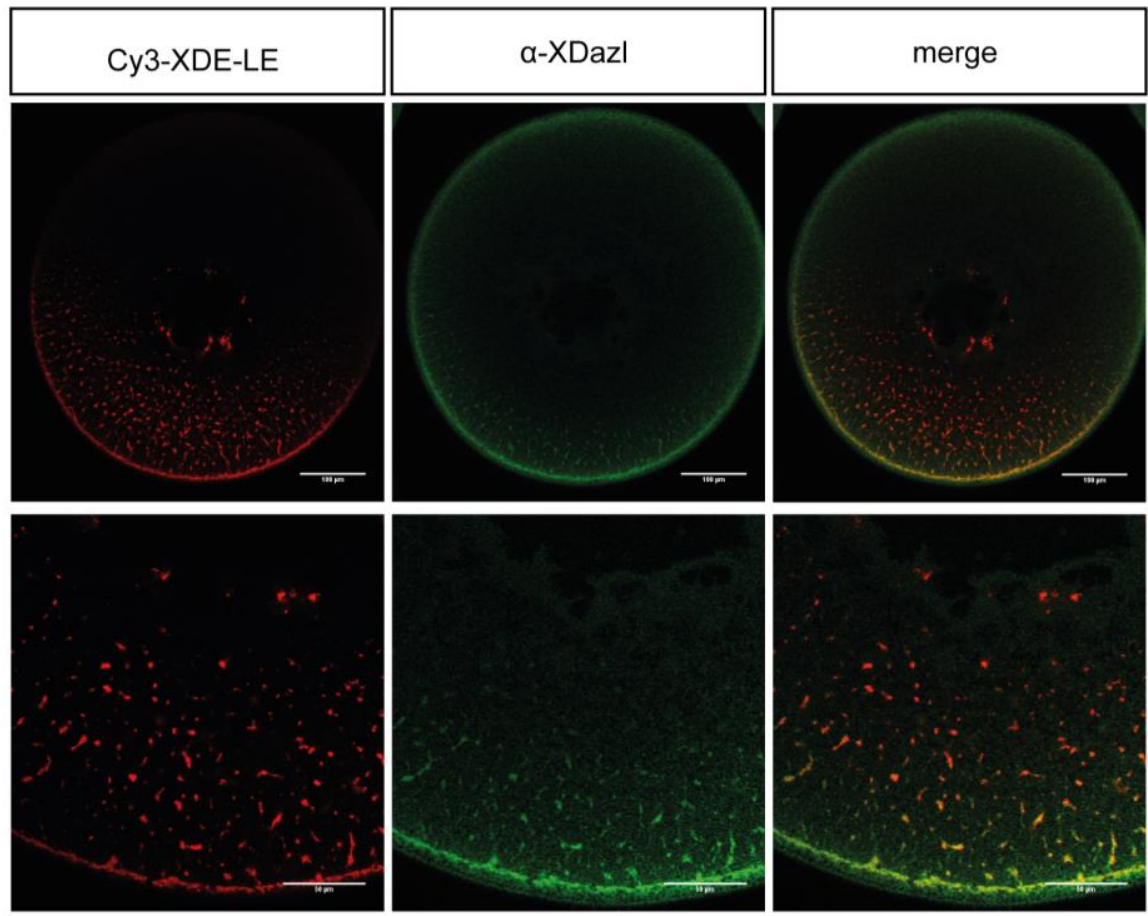

C
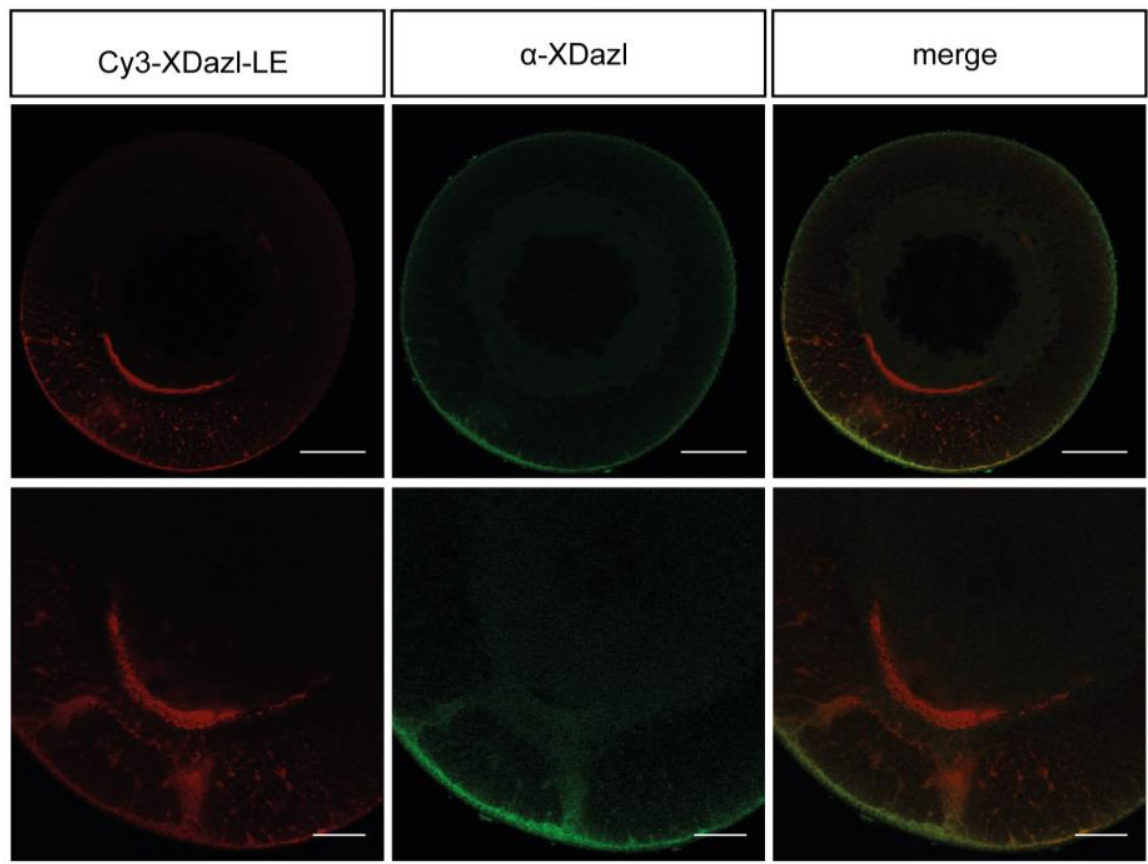

Figure 3.21. XDazl protein colocalizes in Xenopus oocytes with XDE-LE and XDazI-LE RNA.

A) Xenopus stage III oocytes were injected into the nucleus with in vitro transcribed Cy3-labeled RNA, incubated for three days and subjected to immunofluorescence staining against the XDazl protein.

$\mathrm{B}+\mathrm{C})$ Cy3-labeled XDE-LE RNA (B) and XDazl-LE RNA (C) are found to be enriched in stage III oocytes at the vegetal (veg) cortex. Immunofluorescent staining for endogenous XDazl reveals that the protein is enriched in transport particles as well as at the vegetal cortex. Localization of injected Cy3-XDE-LE/XDazl-LE is shown in red, endogenous XDazl is shown in green. Colocalization of endogenous XDazl and injected XDE-LE RNA and XDazl$L E$ is represented in yellow. Lower panel: High-magnification image of the oocyte.

Scale bar represents $100 \mu \mathrm{m}$ in panel for whole oocytes, $50 \mu \mathrm{m}$ in cortex magnification, an animal. 


\subsubsection{The XDazl-LE contains at least three fragments which are sufficient for localization}

As we could show that a shorter $X$ Dazl-LE fragment (XDazl-LE s2) is sufficient to retain PGC specificity and ectopic XDazl mediated somatic reporter stabilization in the embryo (Figure $3.13 \mathrm{~B}+\mathrm{C}$ ) we wanted to know whether shorter XDazl-LE fragments are also adequate to achieve vegetal localization in the oocyte.

For this purpose, we performed localization assays using different Cy3-labeled XDazl-LE subfragments in stage III Xenopus oocytes (Figure 3.22 A). First, we analyzed subfragments that lack regions of different size, from either the 5' end or the 3' end of the XDazl-LE, which were also used in EMSA analysis (Figure 3.10). We found that only those mutants covering the very 5' or 3' 100 nt of the XDazl-LE (XDazI-LE 5'del5 and 3'del5) show a loss of vegetal localization (Figure 3.22 B). Furthermore, we generated a fragment lacking the first 70 and the last $66 \mathrm{nt}$ of the XDazl-LE (XDazl-LE I) as well as subfragments that only partially cover this fragment XDazI-LE $\mathrm{m} 1$ (nt 70-310) and XDazlLE m2 (nt 140-380, data not shown) and tested them in the localization assay. All three subfragments showed proper vegetal localization (Figure 3.22 A). However, as EMSA analysis in Figure 3.10 D suggested XDazl binding to the three non overlapping fragments $X D a z I-L E s 1$, s2 and s3 we assumed that XDazl has different independent binding sites within the LE. Hence, we tested these three fragments in the oocyte localization assay. Indeed, all three fragments showed proper vegetal localization, indicating the existence of at least three independent localization signals within the in silico determined full-length XDazl-LE (Figure $3.22 \mathrm{~A}, \mathrm{~B}$ ). 
A

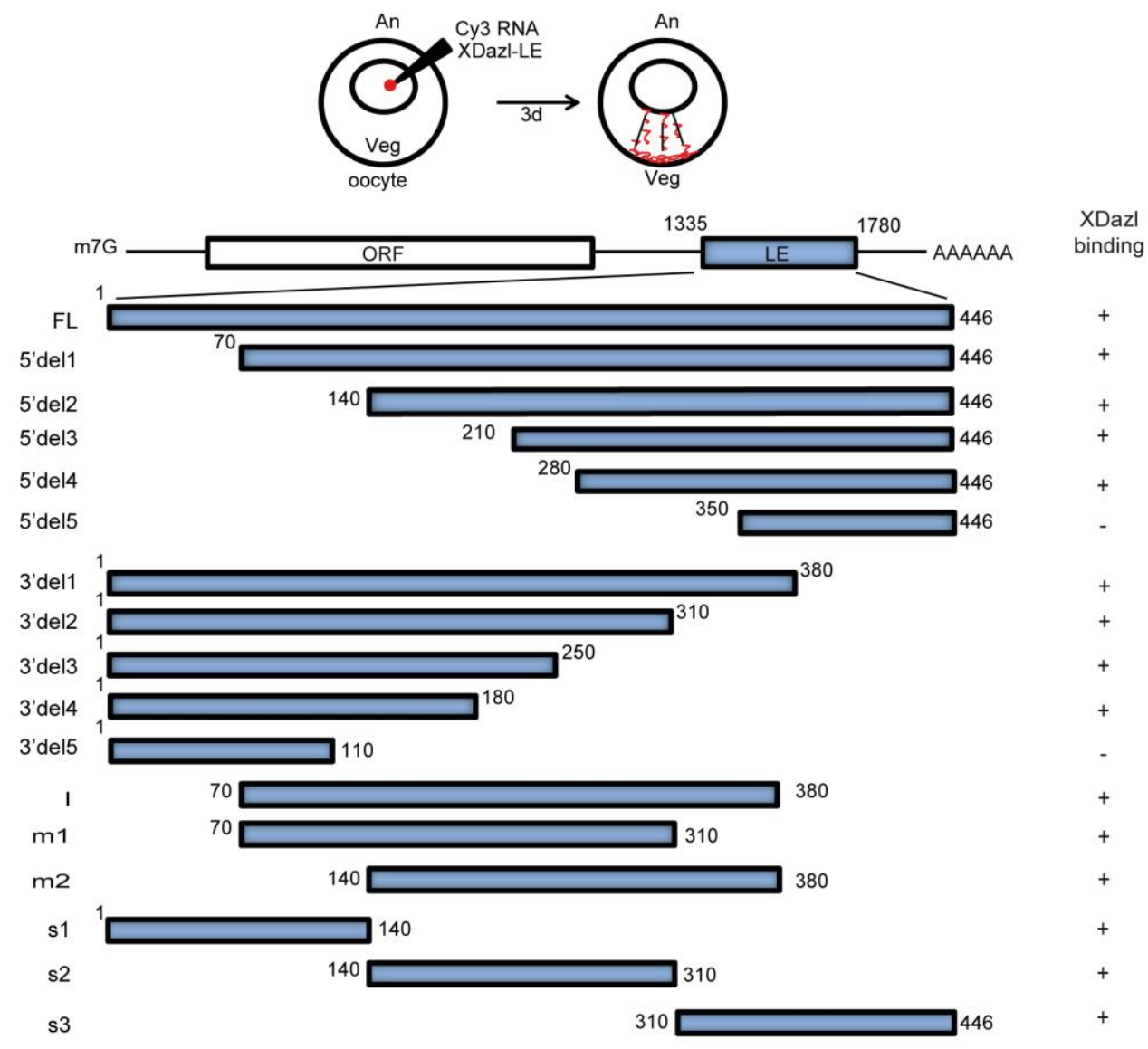

B
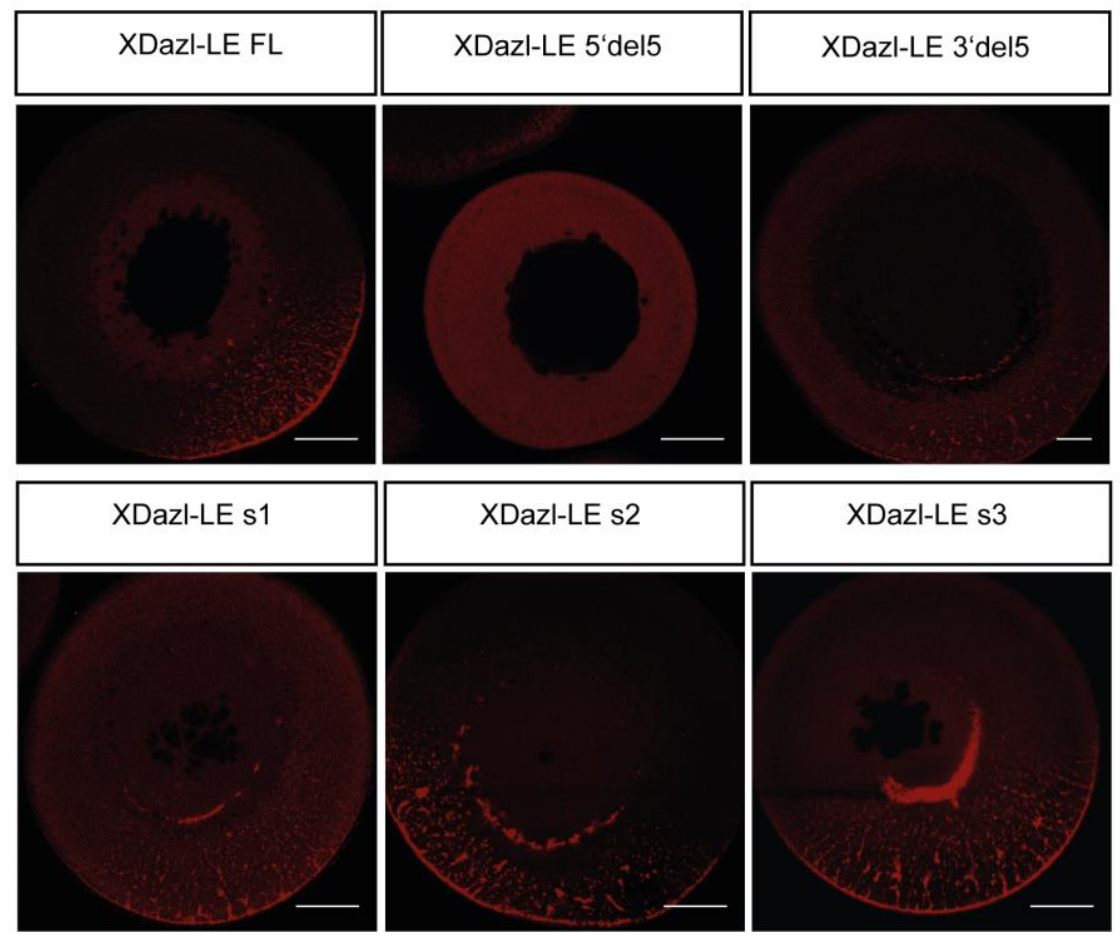

Figure 3.22. The $X D a z I-L E$ contains at least three independent localization signals.

A) Cy3-labeled deletion fragments of the XDazl-LE (depicted in a schematic view) were injected into the nucleus of stage III Xenopus oocytes. After three days of incubation the oocytes were monitored for vegetal localization of the RNA.

B) Visualization of the Cy3-labeled RNA for the different XDazl-LE subfragments, indicating the presence of three independent XDazl localization signals (XDazl-LE s1-s3) and the loss of localization only for XDazl-LE 5 'del5 and 3'del5. Scale bar represents $100 \mu \mathrm{m}$ 
In this study we analyzed the function of the XDazl protein on germ cell specific mRNAs in Xenopus laevis. As indicated above ectopic XDazl expression led to somatic stabilization of germ cell specific reporter RNAs in tadpole stage embryos and endogenous germ cell specific mRNAs in embryos at gastrula stage (paragraph 3.1). In contrast, the injection of XDazl morpholino blocking XDazl translation led to a reduction of PGC number in tadpole embryos (paragraph 3.4). Furthermore, coexpression experiments showed a cooperative function of XDazl and XDE as well as an additive effect of XDazl and ElrB1 in stabilizing germ cell specific reporter RNAs (paragraph 3.1.2). CoIP experiments and EMSAs could show that XDazl mediates stabilization due to direct binding to the germline transcripts. In the same type of experiments the XDazl binding site within the $X D E-L E$ and the $X D a z I-L E$ was defined (paragraph 3.2). In Figure 3.9 it is displayed that XDazl, XDE and ElrB1 protein harbor similar binding sites within the $X D E-L E$. In Xenopus oocytes, the XDazl protein could be shown to colocalize with the $X D E-L E$ as well as with its own $L E$ in transport particles and at the vegetal cortex indicating a possible early function of XDazl. Taken together, these results indicate an important function of XDazl during germ cell development in the embryo and suggest an earlier function of XDazl in the transport or anchoring process of germ cell specific mRNAs in the oocyte. 


\section{DISCUSSION}

In this study, a protective function of XDazl on germ cell specific mRNAs, like $X D E, X p a t$, Xcat2 and its own mRNA was identified in Xenopus embryos. Protection was achieved by specific, direct binding of the XDazl protein to its target mRNAs. Most probably, XDazl prohibits targeting by miRs. The XDazl knock down in embryos leads to a reduction of PGC number but does not influence the level of germ cell specific transcripts after the onset of zygotic transcription. Activation of zygotic XDazl transcription was shown to start at stage 17 with highest expression at stage 26 , the time when germ cells start to migrate actively towards the future gonads. Colocalization of XDazl with vegetally localizing RNAs suggests an additional function in the context of the RNA localization process in oocytes.

\subsection{XDazl as protector of germ cell specific mRNAs in the embryo}

In early embryogenesis maternally provided mRNAs drive the first developmental processes. After the onset of zygotic transcription, these mRNAs are subjected to miR mediated decay (Giraldez 2010). In Xenopus as well as in zebrafish it was shown that germ cell specific transcripts are enriched in PGCs, whereas they are degraded in somatic tissue (Koprunner et al. 2001, Kataoka et al. 2006, Koebernick et al. 2010). Overexpression experiments in Xenopus showed that XDE synergizes with ElrB1 to protect the germline transcript $X D E$ from miR-18 mediated decay (Koebernick et al. 2010). In the same study, LEs residing in the 3' UTR of several different PGC specific mRNAs were shown to be targeted for miR mediated somatic degradation. Therefore, LEs appear to promote two separate functions, germ cell specific RNA stabilization and somatic decay (Koebernick et al. 2010).

Testing for a potential effect of XDazl protein on a $X D E-L E$ reporter revealed its somatic stabilization in a dose dependent manner (Figure 3.1). Thus, overexpression of XDazl is sufficient to protect $X D E-L E$ reporter RNA from somatic clearance. These findings are in accordance with the results from Wiszniak and colleagues, which report that XDazl has a protective function on the germline RNA huB, the zebrafish ortholog of ElrB1 (Wiszniak et al. 2011). Experiments using germline transcript reporter constructs for Xpat-, Xcat2$L E$ and XDazl-LE showed somatic stabilization of these RNAs upon XDazl overexpression (Figure 3.2). Testing for a XDazl function on endogenous mRNAs upon 
ectopic expression indicated a stabilizing effect on all analyzed germ cell specific transcripts (Figure 3.3).

For example the Xpat mRNA was stabilized in XDazl overexpressing embryos. Xpat protein is necessary for germ plasm formation, positioning and maintenance in Xenopus (Machado et al. 2005). Furthermore, Xcat2 mRNA is stabilized upon XDazl ectopic expression. Xcat2 protein plays an important role in Xenopus germ cell development as the inhibition of $X$ cat2 translation leads to failure of PGC migration, followed by their loss through apoptosis. Interestingly, Xcat2 is also required to repress translation of somatic genes in PGCs (Lai et al. 2012). Moreover, XDeadSouth helicase was found to be stabilized by XDazl. It is important for the localization to the germ plasm and proper PGC development in Xenopus (Yamaguchi et al. 2014). Besides, enrichment of Cyclin A1 and $B 2$ was also detectable upon XDazl overexpression (Figure 3.3). Both mRNAs, Cyclin A1 and $B 2$, were shown to be degraded following miR-mediated deadenylation (Lund et al. 2009). Cyclin A1 is a male germ cell-specific cell cycle regulator that is essential for spermatogenesis (Panigrahi et al. 2012), whereas Cyclin B2 is important for germinal vesicle brake down at the end of meiosis I in oocytes (Gui and Homer 2013). However, non-germ cell specific mRNAs, like laminB1 and $\beta$-actin, were not differentially regulated in XDazl overexpressing embryos compared to control embryos (Figure 3.3). These findings indicate that XDazl has a restricted, protective function on germline transcripts. The RRM motif within the XDazl ORF is the important domain for protein-mRNA interaction and it harbors two RNP consensus sequences (Reijo et al. 1995b, Houston and King 2000a, Yen 2004). Deletion of either RNP1 or RNP2 was sufficient to abolish somatic stabilization of the $X D E-L E$ reporter, which in contrast is detectable in embryos overexpressing wild-type XDazl (Figure 3.1, 3.4). These findings are in accordance with results of Maegawa and colleagues, who showed that mutation of already one amino acid (F91) in the RRM in zebrafish Dazl is sufficient to block mRNA recognition (Maegawa et al. 2002).

Our results further provide evidence that XDE and XDazl as well as ElrB1 and XDazl can cooperate with each other to promote germ cell specific mRNA protection (Figure 3.5, 3.6). Similarly, Koebernick et al. revealed a synergism between ElrB1 and XDE in stabilizing XDE-LE mRNA (Koebernick et al. 2010). Nevertheless, ColP experiments could identify only coprecipitation of ElrA and ElrB1 as well as XDazl and ElrA (Figure 3.7). From these experiments we cannot conclude whether the proteins physically interact with each other or if they are incorporated in one RNP complex due to binding to 
the same mRNA. To monitor direct, physical interaction it would be necessary to incubate the protein lysates with RNases to inhibit complex formation on mRNAs. Nevertheless, the coprecipitation of only ElrA with XDazl does not exclude the possibility of the presence of both proteins in a complex with ElrB1, as ElrA and ElrB1 are known to be part of one RNP in oocytes (Arthur et al. 2009). Interestingly, a stabilizing effect of XDazl protein on ElrB1 (huB) mRNA was observed in zebrafish reporter assays (Wiszniak et al. 2011). Moreover, the protein quantities for ElrA and ElrB1 differ in Xenopus embryos as ElrA is expressed ubiquitously and ElrB1 expression is restricted to developing and mature neurons and its presence in ovary and testis (Good 1995, Antic and Keene 1997). Potentially, higher ElrB1 protein amounts are necessary to see coprecipitation of XDazl and ElrB1. Therefore, the ColP could be performed with oocyte lysates containing high endogenous quantities of both proteins. Furthermore, the $X D E$ $L E$ reporter is stabilized in the soma of embryos coexpressing one protein, ElrA, ElrB1 (Koebernick et al. 2010) or XDazl, with the reporter at a time. All these data argue for a possible cooperative or alternate function of ElrA, ElrB1 and XDazl in germline mRNA protection. However the function of ElrA was not addressed in this study.

\subsection{Mechanism of XDazl mediated RNA stabilization}

Overexpression experiments indicated a stabilizing effect of XDazl on its germ cell specific target mRNAs in Xenopus embryos (Figure 3.1, 3.2, 3.3). However, the question of how XDazl fulfills this function was still not absolutely understood. Reporter assays revealed Dazl binding to the 3' UTR of its target mRNAs, such as nanos1, huB and dazl in zebrafish and Ringo/Spy in Xenopus and activation of translation mostly by recruiting PABP to the poly(A) tail (Padmanabhan and Richter 2006, Kedde et al. 2007, Takeda et al. 2009, Wiszniak et al. 2011). ColP experiments performed in our lab indicated direct binding of in vitro translated XDazl protein to the full-length XDE-LE (Löber, 2008). We could confirm these data by using the same method and EMSAs applying recombinant XDazl protein. In order to define the minimal XDazl binding site within the XDE-LE, subfragments of the LE were tested in both assays, CoIP and EMSA, respectively (Appendix, Figure 6.1; Figure 3.8). Deletion of the 5' region of the $X D E-L E$ including and surrounding the miR-18 binding site leads to loss of XDazl binding. Therefore, we can conclude that the $5^{\prime}$ region within the $X D E-L E$ is critical for $X D a z l$ binding. Results presented in Figure 3.9 revealed overlapping binding sites for XDazl, XDE and ElrB1 proteins within the $X D E-L E$ subfragments. Therefore, a $X D E-L E$ deletion mutant shown 
be deficient for ElrB1 binding, vegetal localization and stability in PGCs (Arthur et al. 2009, Koebernick et al. 2010) was used in EMSA for comparative studies. Interestingly, binding to that mutant was completely inhibited for all three proteins, confirming the suggested similarity between the XDE, XDazl and ElrB1 binding sites (Figure 3.9). In this study we could not determine a unique XDazl binding site, which does not affect ElrB1 and XDE binding. Our observations in XDazl overexpression experiments propose a functional link between the proteins ElrA/B1, XDE and XDazl, in germline RNA stabilization. This hypothesis is also supported by the previous findings for the stabilization of the XDE-LE, mediated by ElrB1 and XDE (Koebernick et al. 2010). Furthermore, a possible redundant mechanism could be suggested as the incubation of two out of these three proteins with $X D E-L E$ in the same reaction did not increase binding efficiency or lead to a potential 'supershift' in RNA binding analysis (data not shown). Binding analyses using XDazl protein with LEs of other germ cell specific transcripts indicated direct protein-mRNA interaction with Xpat, XDE and XDazl (Figure 3.11 A, B) Nevertheless, ColP analyses did not provide clear results for the Xcat2-LE (Figure $3.11 \mathrm{~A}$ ), therefore the binding was further examined by EMSA, indicating reduced binding affinity of XDazl to the Xcat2-LE compared to Xpat- or XDE-LE (Figure $3.11 \mathrm{~B}$ ). Thus, a target specific binding affinity of XDazl could be suggested.

Collier reported the presence of multiple Dazl binding sites within target mRNAs, which might be required for translational activation in germ cells (Collier et al. 2005). Correspondingly, the XDazl-LE contains at least three independent XDazl protein binding sites (Figure 3.10 D). These findings are also in accordance with the results from Maegawa et al., revealing that the binding of multiple XDazl proteins increases translational efficiency (Maegawa et al. 2002). Furthermore, a stabilizing effect of XDazl on its own mRNA by poly $(A)$ tail lengthening was described in zebrafish reporter experiments by Takeda and colleagues (Takeda et al. 2009).

XDazl was identified as germ plasm component, which becomes restricted to PGCs during development (Houston et al. 1998). MRNA and protein are maternally supplied in high quantities but become degraded in somatic tissue with the onset of zygotic transcription (Houston et al. 1998, Mita and Yamashita 2000). These results are in accordance with our mRNA and protein expression profiles for XDazl showing XDazl transcripts and protein already in the oocyte and decreasing mRNA levels by stage 9, 
while protein degradation starts at stage 13-14. In tadpole stage 43 embryos, the zygotically translated XDazl protein reappears.

In the early Xenopus embryo, germ cells are transcriptionally silenced. Zygotic transcription in PGCs was shown to start delayed compared to expression in somatic cells (Lai and King 2013). A complete expression profile for Xenopus germline transcripts is still elusive as many of these mRNAs are maternally provided and analyses of transcripts in the zygote can still be influenced by non-degraded maternal mRNAs. An elegant experiment to overcome these difficulties was applied by Yamaguchi and colleagues. To analyze the transcription start of the germ cell specific mRNA $X$ DeadSouth, Xenopus laevis/Xenopus borealis hybrid embryos were generated. As the transcripts for $X D$ DeadSouth of $X$. laevis and $X$. borealis differ in size, the onset of zygotic transcription can be determined by the detection of paternal mRNAs (Yamaguchi et al. 2014). These experiments showed the first $X$ DeadSouth transcripts at embryonic stage 20. In order to analyze the XDazl transcription start we made use of this interspecies cross and fertilized $X$. laevis eggs with $X$. tropicalis sperm (Figure $3.18 \mathrm{~A}$ ). The hybrids were viable until stage 49 but yielded strong developmental defects (Figure $3.18 \mathrm{~B}$ ). MRNA analysis from these hybrid embryos indicated the presence of paternal XDazl transcripts from stage 17 on (Figure $3.18 \mathrm{C}$ ). The expression increases until stage 26. This is the stage, when germ cells start autonomous migration. Possibly, XDazl has a function in the activation of migration. This hypothesis is supported by findings from Houston, who could show that in XDazl depleted embryos germ cells cluster and undergo apoptosis by stage 40 as they are impaired in migration (Houston and King 2000a). Using this interspecies cross, we could also detect XDE transcripts at stage 17 (Figure $3.18 \mathrm{C}$ ). XDE was also described to be involved in germ cell migration, as the knock down leads to the similar phenotype like XDazl knock down (Horvay et al. 2006). In contrast to the results of Yamagushi, who found the first XDeadSouth expression at stage 20, we detected $X$ DeadSouth transcripts already at stage 17 in $X$. laevis $X$. tropicalis hybrids (Figure $3.18 \mathrm{C}$ ). These differences might be caused by the use of sperm from different species, $X$. tropicalis versus $X$. borealis, for the fertilization of $X$. laevis eggs (Yamaguchi et al. 2014).

We used XDazl morpholino injection to block XDazl translation in Xenopus embryos as maternal depletion, used by Houston to achieve the knockdown, represents a complicated host transfer method with lower prospects of success. The morpholino 
induced XDazl knockdown led to reduced XDazl protein levels at stage 13 and a reduction in PGC number at stage 32 (Figure 3.19, 3.20). Nevertheless, the knockdown was not as efficient as the maternal depletion, leading to few up to no PGCs in the embryo (Houston and King 2000a). As knockdown of XDE leads to the same phenotype like XDazl knockdown, clustering and loss of PGCs (Houston and King 2000a, Horvay et al. 2006), we injected both morpholinos but did not see a significant decrease in PGC number compared to embryos injected with only one morpholino. Analysis of transcript levels for XDazl and Xpat in morpholino injected embryos did not indicate any changes on mRNA stability in embryos at stage 14 (Figure $3.20 \mathrm{D}+\mathrm{E}$ ). These findings can be explained by the presence of high quantities of maternally provided XDazl protein (Houston and King 2000a). The supplied protein levels might be sufficient to protect germline transcripts until the translation block, mediated by the morpholino, will have an effect on germ cell specific transcript levels. In future experiments it needs to be analyzed whether the reduction of PGC numbers observed in tadpole embryos correlates with the reduction of Xpat mRNA levels.

Our results showed that XDazl binds its own mRNA (Figure 3.10). Therefore, an autoregulatory mechanism could be suggested, in that increasing XDazl protein levels may reinforce protection of germline $X D a z /$ transcripts. A similar effect was suggested for XDE and its own mRNA (Koebernick et al. 2010). The XDazl protein seems to function as general positive regulator of germ cell specific mRNAs.

\subsection{Degradation of maternally provided germ cell specific mRNAs in the zygote is mediated by miRs}

Xenopus mRNAs, which are enriched in germ cells and become degraded in somatic cells, e.g. $X D E$ and $X D a z l$, are vegetally localized in oocytes and in embryonic germ plasm (MacArthur et al. 2000, Horvay et al. 2006). Sequences located in the 3' UTR were shown to mediate processes like vegetal localization and germ cell enrichment of these mRNAs (Koebernick et al. 2010). Consistent with these data, reporter assays using 3' UTRs of germline transcripts revealed targeting and degradation of these reporters by miRs in the soma of zebrafish and Xenopus embryos by the onset of zygotic transcription (Kataoka et al. 2006, Mishima et al. 2006, Takeda et al. 2009, Koebernick et al. 2010). Furthermore, it has been suggested that PGCs promote expression of germline specific mRNAs by recruiting specific factors to the 3' UTR that increase 
stability or translation (Collier et al. 2005, Mishima et al. 2006, Padmanabhan and Richter 2006). However, analysis of miR mediated effects on the majority of endogenous germ cell specific mRNAs is still elusive in Xenopus. Therefore, we used a siRNA approach described by Lund and colleagues to saturate Ago proteins, which are required for Dicer mediated miR-processing leading to an accumulation of pre-mature miRs in Xenopus embryos (Lund et al. 2011). Ago proteins are present in low amounts in early Xenopus embryos until MBT, so that miR production including siRNA processing is blocked. Lund could show Cyclin B2 enrichment in embryos injected with the siRNA. In uninjected embryos Cyclin B2 is normally degraded by miR-427 targeting upon the onset of zygotic transcription. However, inhibition dissipates upon synthesis of zygotic Ago proteins after MBT. Thus, expression of Ago proteins is controlled developmentally (Lund et al. 2011). Analyses of mRNA levels isolated from siRNA injected embryos indicated a stabilization of germline transcripts, like $X D E$ and $X D a z l$ as well as the known miR target Cyclin B2 (Figure 3.12). As expected, transcripts like lamin B1 are not affected by miRprocessing deficiency (Figure 3.12). Our results demonstrate that the degradation process of maternally provided endogenous germ cell specific mRNAs in the soma is indeed mediated by miRs. The question, which miRs are expressed in PGCs, whether they are involved in the degradation of germline transcripts and how the relative levels of miRs are distributed between germline and somatic cells still remains to be answered. It has been suggested, that the miR associated machinery, including miR processing and miR-mediated degradation, is incomplete in Xenopus PGCs (Yamaguchi et al. 2014) as the Ago proteins are the limiting factor in early embryonic miR mediated decay. Furthermore, a potential delay in Ago expression in PGCs as well as the zygotic expression of transcriptional/translational regulator genes has been proposed to be the reason for germ cell specific mRNA stabilization (Venkatarama et al. 2010). If so, it is a reasonable explanation for the low expression levels of mature miR-427 in PGCs detected by Yamaguchi and colleagues (Yamaguchi et al. 2014).

In this study, a minimal XDazl-LE fragment (XDazl-LE s2, LE nt 140-310) was found to fulfill all functional characteristics, like vegetal localization, germline restriction and XDazl mediated stabilization (Figure 3.13, 3.22). Morpholino protection assays, blocking potential miR target sites within the LE, identified the 3' region of the XDazl-LE s2 subfragment as most critical for miR mediated decay (Figure 3.14). An in silico analysis of the full length $X D a z l-L E$ via the miRanda program identified four potential candidate miR target sites in the subfragment XDazl-LE s2 and only one miR target site in the 3' region for miR-489 (Figure 3.14, Figure 3.15), which only partially maps to the $s 2$ 
subfragment. MiR-489 is expressed in Xenopus from stage 10 to stage 17, but nothing is known about its function. In mouse, miR-489 is involved in maintaining the quiescent state of an adult stem-cell population (Cheung et al. 2012). Furthermore, a mir-18a/b binding site was proposed within the XDazl-LE s2 fragment by in silico analysis. MiR$18 \mathrm{a} / \mathrm{b}$ was found to target the XDE-LE for somatic degradation in Xenopus (Koebernick et al. 2010).

In mice, mir-18a was shown to directly target HSF2 (heat shock factor 2), a transcription factor that controls the expression of genes required for spermatogenesis. HSF2 knockout mice exhibit small, morphologically abnormal testes with fewer spermatids and severe sperm abnormalities (Bjork et al. 2010). EMSA binding analysis (Figure 3.16) indicated the region blocked by $\mathrm{MO} 2$, corresponding to the miR-18 binding site in the LE, as most critical for XDazl binding (Figure 3.15, 3.16). Another predicted binding site, for miR-34/449, was fully blocked by MO4 and partially by MO5 binding in the morpholino protection assay leading to moderate somatic reporter stabilization (Figure $3.14 \mathrm{~B}+\mathrm{C}$ ). Published data revealed that the miR-449 and miR-34b/c function redundantly in regulating male germ cell development in murine testes (Bao et al. 2012). Recently, Song and colleagues demonstrated that miR-34/449 regulate ciliogenesis and miR$34 / 449$ knockout mice yield infertility phenotypes (Song et al. 2014). The mir-449 was also found as regulator of multiciliated differentiation, such that a role in formation of flagella in spermatids is very likely. High expression levels of miR-449 were found in testicular tissue suggesting that it might have a function in gem cell development (Lize et al. 2011). All these connections of miR-34/449 to germ cell development make it an interesting candidate to test for. Functional analysis on the XDazl-LE s2 reporter using 2'O-Methyl-Oligonucleotides (2'OMeO), inhibiting miRs by direct binding, need to be performed to clarify the involvement of these distinct miRs in XDazI-LE s2 mediated degradation.

\subsection{XDazl function in vegetal transport in the oocyte}

XDazl protein was shown to bind directly to the XDE-LE RNA in vitro (Figure 3.8, Löber, 2008). Moreover, it is expressed in oocytes and germ cells (Houston and King 2000a, Mita and Yamashita 2000). Association of XDazl mRNA with the germ plasm in Xenopus oocytes and the protein expression during oocyte stages (Figure 3.17) could point to an early function of XDazl during oogenesis (Houston and King 2000a, Mita and Yamashita 2000). For the Elav-proteins it was shown that they synergize with XDE to counteract 
miR-18 mediated decay of XDE RNA (Koebernick et al. 2010). Interestingly, Elav proteins are also involved in RNA localization in the oocyte (Arthur et al. 2009). Therefore, it might be possible that these proteins bind their target mRNAs during the vegetal transport in the oocyte and remain associated during embryogenesis. A similar mechanism of XDazl mediated target protection could be suggested. Immunostaining identified the XDazl protein to colocalize with XDE-LE or XDazl-LE in transport particles and at the vegetal cortex of oocytes (Figure $3.21 \mathrm{~B}$ ). As the loss of the overlapping XDazl, XDE and ElrB1 binding sites (Figure 3.9) within the XDE-LE (XDE-LE mut2b) (Arthur et al. 2009) leads to inhibited localization in oocytes and loss of germ cell specificity in the embryo a potential function of all three proteins in vegetal transport as well as in germ cell determination can be suggested. To further analyze a possible XDazl function during vegetal transport and anchoring, ColP experiments with oocyte extracts will be performed in the future to examine mRNAs and proteins bound to XDazl.

\subsection{Connection of the XDazl function in the oocyte and the embryo}

Three independent fragments of the XDazl-LE (XDazl-LE s1-s3) localize to the vegetal pole in oocytes indicating the presence of three localization signals (Figure 3.22). As the fragments $s 1$ and s2 are somatically degraded (s3 only partially), when injected into the embryo (Figure 3.13), this suggests the presence of different miR-target sites, which mediate the somatic degradation. In silico analysis of the XDazl-LE identified potential miR target sites in all three subfragments as depicted in Figure 4.1 (red). Therefore, we can hypothesize that the XDazl protein has different binding sites within its localization element to protect the mRNA against targeting by different miRs.

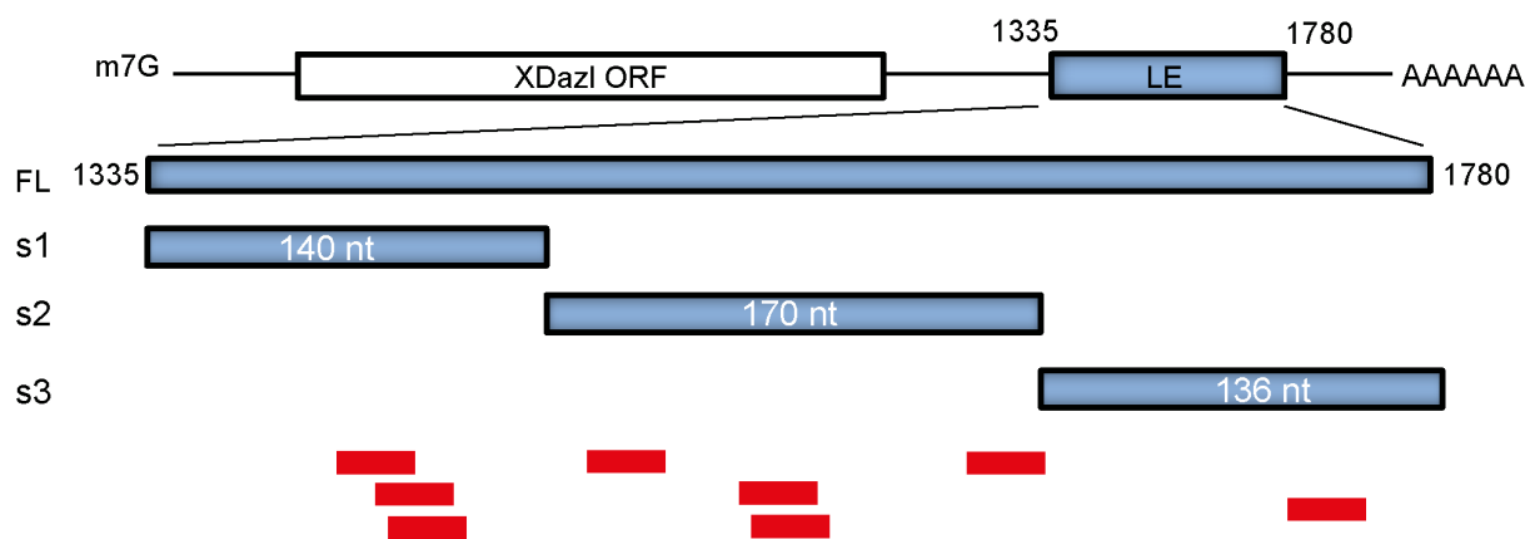

Figure 4.1. XDazl full length localization element contains different potential miR-binding sites.

The full length $X$ Dazl-LE (FL) was scanned for potential miR binding sites using in silico analysis (Enright et al. 2003), which are depicted as red bars. Also within XDazl-LE subfragments s1-s3 target sites for different miRs are present. 
XDazl mRNA could be detected in both, oocytes and embryos associated with the germ plasm. In general, localization in the oocyte should ensure the presence of all germ cell specific mRNAs in the region of the germ plasm, which will give rise to germ cells in the developing embryo. Nevertheless, in situ hybridization of $X D E$ mRNA revealed presence of transcripts outside of the germ plasm, may be due to localization via the late pathway and the anchoring in a broader region of the vegetal cortex. These transcripts outside of the germ plasm need to be degraded as their presence in somatic cells would lead to developmental failures in the embryo. To avoid degradation of germline transcripts in PGCs by the global expression of miRs, protective proteins need to counteract the miR function. Until now we can conclude from our experiments that XDazl, XDE and also ElrB1 are involved in the protection of germline transcripts. We suggest a model, where ElrB1 and XDazl are attached to the target mRNA, e.g. XDE, already in the oocyte and the RNP complex remains intact until the PGCs are specified. In the embryo, XDE protein can bind to the RNP counteracting the targeting by miR-18. The protection of the germ cell specific mRNA leads probably to increased translation of XDE proteins, which in turn can stabilize its own mRNA by direct binding to the RNP in an autoregulatory mechanism (Figure 4.2).
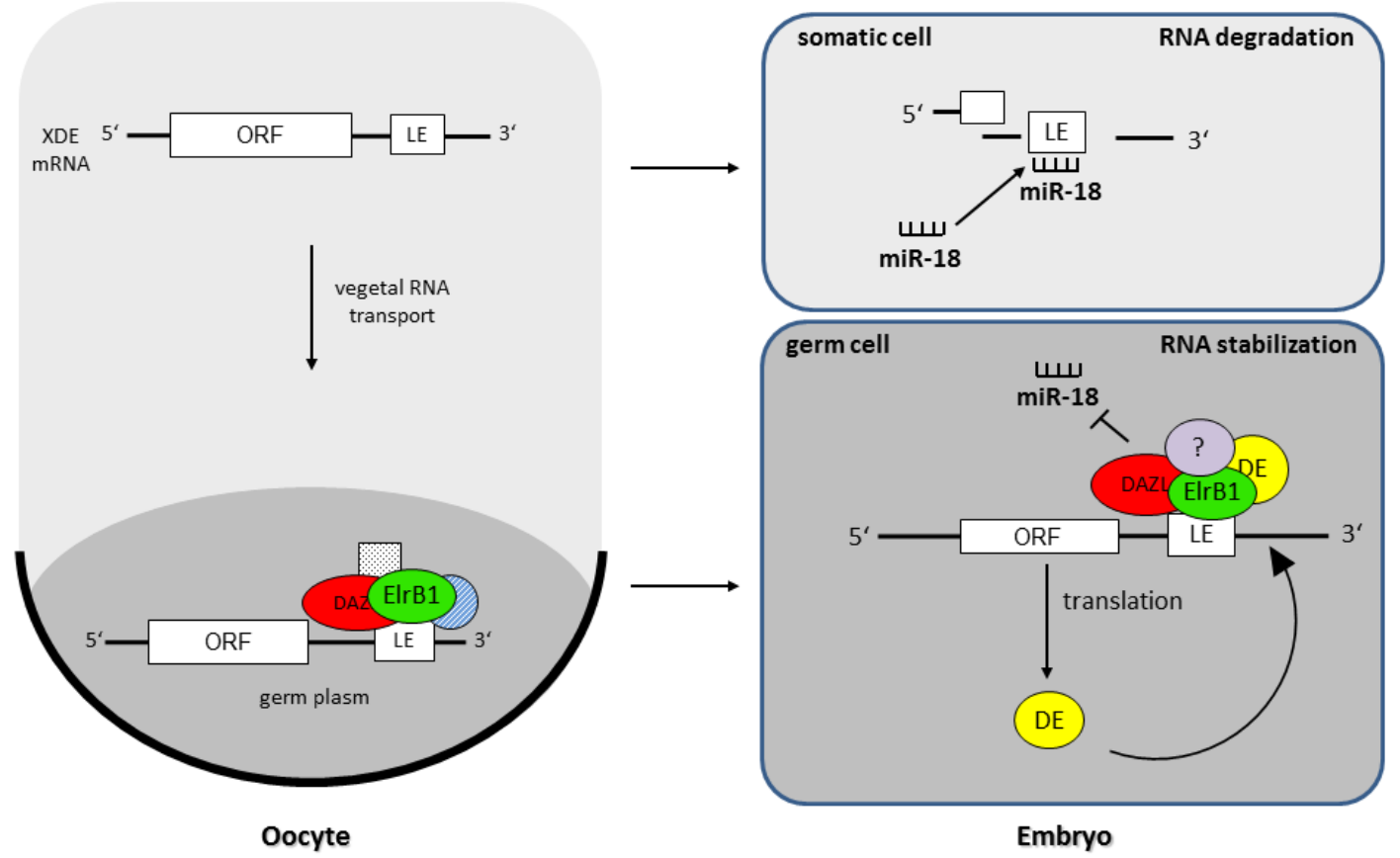

Figure 4.2. Functional connections between vegetal mRNA transport in oocytes and germ cell specific mRNA protection in the embryo.

Elr-type and XDazl proteins are involved in the vegetal transport of $X D E m R N A$ by binding to the $X D E$ localization element $(X D E-L E)$ in Xenopus oocytes. This mRNA is degraded in somatic cells by miR-18 mediated decay in the embryo. In germ cells, Elr-type and XDazl protein binding leads to protection against this decay. Translated XDE can bind to the protection complex and leads to a cooperative effect (after M. Claussen, P. Arthur, K. Koebernick, (Arthur et al. 2009, Claussen and Pieler 2010, Koebernick et al. 2010)). 
A similar autoregulatory mechanism might be responsible for the regulation of translation of XDazl. Binding of the XDazl protein to its own mRNA could counteract the targeting of miRs within its own mRNA, leading to increased translation. However, it is also likely that further proteins are involved in the protection mechanism, as in ColP experiments also ElrA was found to coprecipitate with XDazl in Xenopus stages when mRNA protection against miR mediated decay is necessary. To date it is still unclear whether only one of the proteins recruits the target mRNA or whether different proteins can bind to the mRNA simultaneously. Future experiments will be performed to address this question. 


\section{CONCLUSION}

In this study, we showed that XDazl has a stabilizing function on multiple germ cell specific transcripts. Experiments suggest a cooperative interaction of the XDazl, XDE and ElrB1 protein in this protective mechanism. Most likely, the RNP complex including XDazl, ElrA/B1 and the germline transcript is established already in the oocyte as we could see colocalization of XDazl and its target mRNAs. In the embryo, maternally supplied PGC specific transcripts are degraded by miR mediated decay in the soma but are protected in germ cells due to binding of specific proteins, like XDazl. The presence of several XDazl binding sites within its own mRNA suggests that PGC-specific transcript protection is regulated specifically as also different miRs are responsible for degradation of the XDazl mRNA. We further showed that the persistence of XDazl mRNA in PGCs was caused by zygotic expression before and at the stage when active migration of germ cells starts. These findings demonstrate the different important functions of XDazl in the process of germ cell development in Xenopus laevis. 


\section{REFERENCES}

Abe, R., K. Yamamoto, and H. Sakamoto. 1996. Target specificity of neuronal RNA-binding protein, Mel-N1: Direct binding to the $3^{\prime}$ untranslated region of its own mRNA. Nucleic acids research 24: 2011-2016.

Ali, S., N. Karki, C. Bhattacharya, R. Zhu, D. A. MacDuff, M. D. Stenglein, A. J. Schumacher, Z. L. Demorest, R. S. Harris, A. Matin, and S. Aggarwal. 2013. APOBEC3 inhibits DEAD-END function to regulate microRNA activity. BMC Mol Biol 14: 16.

Allison, R., K. Czaplinski, A. Git, E. Adegbenro, F. Stennard, E. Houliston, and N. Standart. 2004. Two distinct Staufen isoforms in Xenopus are vegetally localized during oogenesis. Rna 10: 1751-1763.

Antic, D., and J. D. Keene. 1997. Embryonic lethal abnormal visual RNA-binding proteins involved in growth, differentiation, and posttranscriptional gene expression. American journal of human genetics $61: 273-278$.

Arthur, P. K. 2008. Identification and Functional Characterization of Trans-acting Factors

Involved in Vegetal mRNA Localization in Xenopus Oocytes. Dissertation, Georg-AugustUniversität Göttingen.

Arthur, P. K., M. Claussen, S. Koch, K. Tarbashevich, O. Jahn, and T. Pieler. 2009. Participation of Xenopus Elr-type proteins in vegetal mRNA localization during oogenesis. The Journal of biological chemistry 284: 19982-19992.

Bagga, S., J. Bracht, S. Hunter, K. Massirer, J. Holtz, R. Eachus, and A. E. Pasquinelli. 2005. Regulation by let-7 and lin-4 miRNAs results in target mRNA degradation. Cell 122: 553-563.

Baneyx, F. 1999. Recombinant protein expression in Escherichia coli. Curr Opin Biotechnol 10: 411-421.

Bao, J., D. Li, L. Wang, J. Wu, Y. Hu, Z. Wang, Y. Chen, X. Cao, C. Jiang, W. Yan, and C. Xu. 2012. MicroRNA-449 and microRNA-34b/c function redundantly in murine testes by targeting E2F transcription factor-retinoblastoma protein (E2F-pRb) pathway. The Journal of biological chemistry 287: 21686-21698.

Bartel, D. P. 2004. MicroRNAs: genomics, biogenesis, mechanism, and function. Cell 116: 281 297.

Bartel, D. P. 2009. MicroRNAs: target recognition and regulatory functions. Cell 136: 215-233.

Bauermeister, D., M. Claußen, and T. Pieler. 2014. Biochemical Aspects of Subcellular RNA Transport and Localization, pp. pp 293-308 Chemical Biology of Nucleic Acids.

Betel, D., M. Wilson, A. Gabow, D. S. Marks, and C. Sander. 2008. The microRNA.org resource: targets and expression. Nucleic acids research 36: D149-153.

Betel, D., A. Koppal, P. Agius, C. Sander, and C. Leslie. 2010. Comprehensive modeling of microRNA targets predicts functional non-conserved and non-canonical sites. Genome biology 11: R90.

Betley, J. N., M. C. Frith, J. H. Graber, S. Choo, and J. O. Deshler. 2002. A ubiquitous and conserved signal for RNA localization in chordates. Current biology : CB 12: 1756-1761.

Bettegowda, A., and G. W. Smith. 2007. Mechanisms of maternal mRNA regulation: implications for mammalian early embryonic development. Frontiers in bioscience : a journal and virtual library 12: 3713-3726.

Bhattacharyya, S. N., R. Habermacher, U. Martine, E. I. Closs, and W. Filipowicz. 2006. Relief of microRNA-mediated translational repression in human cells subjected to stress. Cell 125: 1111-1124.

Bjork, J. K., A. Sandqvist, A. N. Elsing, N. Kotaja, and L. Sistonen. 2010. miR-18, a member of Oncomir-1, targets heat shock transcription factor 2 in spermatogenesis. Development 137: 3177-3184.

Blaser, H., S. Eisenbeiss, M. Neumann, M. Reichman-Fried, B. Thisse, C. Thisse, and E. Raz. 2005. Transition from non-motile behaviour to directed migration during early PGC development in zebrafish. Journal of cell science 118: 4027-4038.

Bontems, F., A. Stein, F. Marlow, J. Lyautey, T. Gupta, M. C. Mullins, and R. Dosch. 2009. Bucky ball organizes germ plasm assembly in zebrafish. Current biology : CB 19: 414422. 
Bradford, M. M. 1976. A rapid and sensitive method for the quantitation of microgram quantities of protein utilizing the principle of protein-dye binding. Anal Biochem 72: 248-254.

Brangwynne, C. P., C. R. Eckmann, D. S. Courson, A. Rybarska, C. Hoege, J. Gharakhani, F. Julicher, and A. A. Hyman. 2009. Germline P granules are liquid droplets that localize by controlled dissolution/condensation. Science 324: 1729-1732.

Brekhman, V., J. Itskovitz-Eldor, E. Yodko, M. Deutsch, and J. Seligman. 2000. The DAZL1 gene is expressed in human male and female embryonic gonads before meiosis. Molecular human reproduction 6: 465-468.

Brook, M., J. W. Smith, and N. K. Gray. 2009. The DAZL and PABP families: RNA-binding proteins with interrelated roles in translational control in oocytes. Reproduction 137: 595617.

Campos, A. R., D. Grossman, and K. White. 1985. Mutant Alleles at the Locus Elav in Drosophila-Melanogaster Lead to Nervous-System Defects - a Developmental GeneticAnalysis. J Neurogenet 2: 197-218.

Cheung, T. H., N. L. Quach, G. W. Charville, L. Liu, L. Park, A. Edalati, B. Yoo, P. Hoang, and T. A. Rando. 2012. Maintenance of muscle stem-cell quiescence by microRNA-489. Nature 482: 524-528.

Chung, Y. D., H. C. Kwon, K. W. Chung, S. J. Kim, K. Kim, and C. C. Lee. 1996. Identification of ovarian enhancer-binding factors which bind to ovarian enhancer 1 of the Drosophila genes yp1 and yp2. Molecular \& general genetics : MGG 251: 347-351.

Claussen, M., and T. Pieler. 2010. Identification of vegetal RNA-localization elements in Xenopus oocytes. Methods 51: 146-151.

Collier, B., B. Gorgoni, C. Loveridge, H. J. Cooke, and N. K. Gray. 2005. The DAZL family proteins are PABP-binding proteins that regulate translation in germ cells. Embo $\mathrm{J} 24$ : 2656-2666.

Cox, D. N., A. Chao, J. Baker, L. Chang, D. Qiao, and H. F. Lin. 1998. A novel class of evolutionarily conserved genes defined by piwi are essential for stem cell self-renewal. Genes \& development 12: 3715-3727.

Czaplinski, K., T. Kocher, M. Schelder, A. Segref, M. Wilm, and I. W. Mattaj. 2005. Identification of $40 \mathrm{LoVe}$, a Xenopus hnRNP D family protein involved in localizing a TGFbeta-related mRNA during oogenesis. Developmental cell 8: 505-515.

Dai, T., Y. Vera, E. C. Salido, and P. H. Yen. 2001. Characterization of the mouse Dazap1 gene encoding an RNA-binding protein that interacts with infertility factors DAZ and DAZL. BMC Genomics 2: 6.

Deshler, J. O., M. I. Highett, T. Abramson, and B. J. Schnapp. 1998. A highly conserved RNAbinding protein for cytoplasmic mRNA localization in vertebrates. Current biology : CB 8: 489-496.

Devaux, A., L. J. Colegrove-Otero, and N. Standart. 2006. Xenopus ElrB, but not ElrA, binds RNA as an oligomer: Possible role of the linker. Febs Lett 580: 4947-4952.

Draper, B. W., C. M. McCallum, and C. B. Moens. 2007. nanos1 is required to maintain oocyte production in adult zebrafish. Developmental biology 305: 589-598.

Dumont, J. N. 1972. Oogenesis in Xenopus laevis (Daudin). I. Stages of oocyte development in laboratory maintained animals. Journal of morphology 136: 153-179.

Dzementsei, A. 2013. Role of cellular dynamics, adhesion and polarity in the context of primordial germ cell migration in Xenopus laevis embryos.

Eberhart, C. G., J. Z. Maines, and S. A. Wasserman. 1996. Meiotic cell cycle requirement for a fly homologue of human Deleted in Azoospermia. Nature 381: 783-785.

Elinson, R. P., M. L. King, and C. Forristall. 1993. Isolated Vegetal Cortex from XenopusOocytes Selectively Retains Localized Messenger-Rnas. Developmental biology 160: 554-562.

Elliott, D. J., M. R. Millar, K. Oghene, A. Ross, F. Kiesewetter, J. Pryor, M. Mclntyre, T. B. Hargreave, P. T. Saunders, P. H. Vogt, A. C. Chandley, and H. Cooke. 1997. Expression of RBM in the nuclei of human germ cells is dependent on a critical region of the $\mathrm{Y}$ chromosome long arm. Proceedings of the National Academy of Sciences of the United States of America 94: 3848-3853.

Enright, A. J., B. John, U. Gaul, T. Tuschl, C. Sander, and D. S. Marks. 2003. MicroRNA targets in Drosophila. Genome biology 5: R1. 
Ephrussi, A., and R. Lehmann. 1992. Induction of Germ-Cell Formation by Oskar. Nature 358: 387-392.

Extavour, C. G., and M. Akam. 2003. Mechanisms of germ cell specification across the metazoans: epigenesis and preformation. Development 130: 5869-5884.

Fabian, M. R., N. Sonenberg, and W. Filipowicz. 2010. Regulation of mRNA translation and stability by microRNAs. Annual review of biochemistry 79: 351-379.

Fan, X. H. C., and J. A. Steitz. 1998. HNS, a nuclear-cytoplasmic shuttling sequence in HuR. Proceedings of the National Academy of Sciences of the United States of America 95: 15293-15298.

Fisher, M. P., and C. W. Dingman. 1971. Role of molecular conformation in determining the electrophoretic properties of polynucleotides in agarose-acrylamide composite gels. Biochemistry 10: 1895-1899.

Foresta, C., A. Ferlin, A. Rossi, E. Salata, and A. Tessari. 2002. [Alteration of spermatogenesis and $Y$ chromosome microdelations. Analysis of the DAZ gene family]. Minerva endocrinologica 27: 193-207.

Gagnon, J. A., and K. L. Mowry. 2011. Visualization of mRNA localization in Xenopus oocytes. Methods in molecular biology 714: 71-82.

Gao, M., and A. L. Arkov. 2013. Next generation organelles: structure and role of germ granules in the germline. Molecular reproduction and development 80: 610-623.

Ghosh, D., and G. Seydoux. 2008. Inhibition of transcription by the Caenorhabditis elegans germline protein PIE-1: genetic evidence for distinct mechanisms targeting initiation and elongation. Genetics 178: 235-243.

Giraldez, A. J. 2010. microRNAs, the cell's Nepenthe: clearing the past during the maternal-tozygotic transition and cellular reprogramming. Current opinion in genetics \& development 20: 369-375

Giraldez, A. J., Y. Mishima, J. Rihel, R. J. Grocock, S. Van Dongen, K. Inoue, A. J. Enright, and A. F. Schier. 2006. Zebrafish MiR-430 promotes deadenylation and clearance of maternal mRNAs. Science 312: 75-79.

Good, P. J. 1995. A Conserved Family of Elav-Like Genes in Vertebrates. Proceedings of the National Academy of Sciences of the United States of America 92: 4557-4561.

Gotoh, Y., N. Masuyama, K. Dell, K. Shirakabe, and E. Nishida. 1995. Initiation of Xenopus oocyte maturation by activation of the mitogen-activated protein kinase cascade. The Journal of biological chemistry 270: 25898-25904.

Gui, L., and H. Homer. 2013. Hec1-dependent cyclin B2 stabilization regulates the G2-M transition and early prometaphase in mouse oocytes. Developmental cell 25: 43-54.

Gustafson, E. A., and G. M. Wessel. 2010. Vasa genes: emerging roles in the germ line and in multipotent cells. BioEssays : news and reviews in molecular, cellular and developmental biology 32: 626-637.

Haag, E. S. 2001. Rolling back to BOULE. Proceedings of the National Academy of Sciences of the United States of America 98: 6983-6985.

Hanyu-Nakamura, K., H. Sonobe-Nojima, A. Tanigawa, P. Lasko, and A. Nakamura. 2008. Drosophila Pgc protein inhibits P-TEFb recruitment to chromatin in primordial germ cells. Nature 451: 730-U737.

Harland, R. M. 1991. In situ hybridization: an improved whole-mount method for Xenopus embryos. Methods in cell biology 36: 685-695.

Hayashi, Y., M. Hayashi, and S. Kobayashi. 2004. Nanos suppresses somatic cell fate in Drosophila germ line. Proceedings of the National Academy of Sciences of the United States of America 101: 10338-10342.

Hogan, B. L. 1996. Bone morphogenetic proteins in development. Current opinion in genetics \& development 6: 432-438.

Hollemann, T., and T. Pieler. 1999. Xpitx-1: a homeobox gene expressed during pituitary and cement gland formation of Xenopus embryos. Mechanisms of development 88: 249-252.

Horvay, K., M. Claussen, M. Katzer, J. Landgrebe, and T. Pieler. 2006. Xenopus Dead end mRNA is a localized maternal determinant that serves a conserved function in germ cell development. Developmental biology 291: 1-11.

Houston, D. W., and M. L. King. 2000a. A critical role for Xdazl, a germ plasm-localized RNA, in the differentiation of primordial germ cells in Xenopus. Development 127: 447-456. 
Houston, D. W., and M. L. King. 2000b. Germ plasm and molecular determinants of germ cell fate. Curr Top Dev Biol 50: 155-181.

Houston, D. W., J. Zhang, J. Z. Maines, S. A. Wasserman, and M. L. King. 1998. A Xenopus DAZ-like gene encodes an RNA component of germ plasm and is a functional homologue of Drosophila boule. Development 125: 171-180.

Houwing, S., L. M. Kamminga, E. Berezikov, D. Cronembold, A. Girard, H. van den Elst, D. V. Filippov, H. Blaser, E. Raz, C. B. Moens, R. H. Plasterk, G. J. Hannon, B. W. Draper, and R. F. Ketting. 2007. A role for Piwi and piRNAs in germ cell maintenance and transposon silencing in Zebrafish. Cell 129: 69-82.

Hsu, L. C., H. Y. Chen, Y. W. Lin, W. C. Chu, M. J. Lin, Y. T. Yan, and P. H. Yen. 2008. DAZAP1, an hnRNP protein, is required for normal growth and spermatogenesis in mice. Rna 14: 1814-1822.

Hudson, C., and H. R. Woodland. 1998a. Xpat, a gene expressed specifically in germ plasm and primordial germ cells of Xenopus laevis. Mechanisms of development 73: 159-168.

Hudson, C., and H. R. Woodland. 1998b. Xpat, a gene expressed specifically in germ plasm and primordial germ cells of Xenopus laevis. Mechanisms of development 73: 159-168.

Huettner, A. F. 1930. Recent Criticisms Concerning Meiosis in Drosophila Melanogaster. Science 71: 241-243.

IIImense.K, and A. P. Mahowald. 1974. Transplantation of Posterior Polar Plasm in Drosophila Induction of Germ-Cells at Anterior Pole of Egg. Proceedings of the National Academy of Sciences of the United States of America 71: 1016-1020.

IIImensee, K., and A. P. Mahowald. 1976. Autonomous Function of Germ Plasm in a Somatic Region of Drosophila Egg. Exp Cell Res 97: 127-140.

Jain, R. G., L. G. Andrews, K. M. McGowan, P. H. Pekala, and J. D. Keene. 1997. Ectopic expression of Hel-N1, an RNA-binding protein, increases glucose transporter (GLUT1) expression in 3T3-L1 adipocytes. Mol Cell Biol 17: 954-962.

Jenkins, H. T., B. Malkova, and T. A. Edwards. 2011. Kinked beta-strands mediate high-affinity recognition of mRNA targets by the germ-cell regulator DAZL. Proceedings of the National Academy of Sciences of the United States of America 108: 18266-18271.

Kataoka, K., T. Yamaguchi, H. Orii, A. Tazaki, K. Watanabe, and M. Mochii. 2006. Visualization of the Xenopus primordial germ cells using a green fluorescent protein controlled by cis elements of the 3 ' untranslated region of the DEADSouth gene. Mechanisms of development 123: 746-760.

Kawasaki, I., Y. H. Shim, J. Kirchner, J. Kaminker, W. B. Wood, and S. Strome. 1998. PGL-1, a predicted RNA-binding component of germ granules, is essential for fertility in C. elegans. Cell 94: 635-645.

Kedde, M., M. J. Strasser, B. Boldajipour, J. A. Oude Vrielink, K. Slanchev, C. le Sage, R. Nagel, P. M. Voorhoeve, J. van Duijse, U. A. Orom, A. H. Lund, A. Perrakis, E. Raz, and R. Agami. 2007. RNA-binding protein Dnd1 inhibits microRNA access to target mRNA. Cell 131: 1273-1286.

Keene, J. D. 1999. Why is Hu where? Shuttling of early-response-gene messenger RNA subsets. Proceedings of the National Academy of Sciences of the United States of America 96: 57.

Kim, B., H. J. Cooke, and K. Rhee. 2012. DAZL is essential for stress granule formation implicated in germ cell survival upon heat stress. Development 139: 568-578.

King, M. L., T. J. Messitt, and K. L. Mowry. 2005. Putting RNAs in the right place at the right time: RNA localization in the frog oocyte. Biol Cell 97: 19-33.

Kloc, M., and L. D. Etkin. 1995. 2 Distinct Pathways for the Localization of Rnas at the Vegetal Cortex in Xenopus Oocytes. Development 121: 287-297.

Kloc, M., N. R. Zearfoss, and L. D. Etkin. 2002. Mechanisms of subcellular mRNA localization. Cell 108: 533-544.

Kloc, M., S. Bilinski, A. P. Chan, L. H. Allen, N. R. Zearfoss, and L. D. Etkin. 2001. RNA localization and germ cell determination in Xenopus. International review of cytology 203: 63-91.

Koebernick, K., J. Loeber, P. K. Arthur, K. Tarbashevich, and T. Pieler. 2010. Elr-type proteins protect Xenopus Dead end mRNA from miR-18-mediated clearance in the soma. Proceedings of the National Academy of Sciences of the United States of America 107: 16148-16153. 
Koprunner, M., C. Thisse, B. Thisse, and E. Raz. 2001. A zebrafish nanos-related gene is essential for the development of primordial germ cells. Genes \& development 15: 28772885.

Kress, T. L., Y. J. Yoon, and K. L. Mowry. 2004. Nuclear RNP complex assembly initiates cytoplasmic RNA localization. The Journal of cell biology 165: 203-211.

Kroll, T. T., L. B. Swenson, E. I. Hartland, D. D. Snedden, H. V. Goodson, and P. W. Huber. 2009. Interactions of 40LoVe within the ribonucleoprotein complex that forms on the localization element of Xenopus Vg1 mRNA. Mechanisms of development 126: 523-538.

Kunwar, P. S., D. E. Siekhaus, and R. Lehmann. 2006. In vivo migration: a germ cell perspective. Annual review of cell and developmental biology 22: 237-265.

Laemmli, U. K. 1970. Cleavage of structural proteins during the assembly of the head of bacteriophage T4. Nature 227: 680-685.

Lai, F., and M. L. King. 2013. Repressive translational control in germ cells. Molecular reproduction and development 80: 665-676.

Lai, F., A. Singh, and M. L. King. 2012. Xenopus Nanos1 is required to prevent endoderm gene expression and apoptosis in primordial germ cells. Development 139: 1476-1486.

Lai, F., Y. Zhou, X. Luo, J. Fox, and M. L. King. 2011. Nanos1 functions as a translational repressor in the Xenopus germline. Mechanisms of development 128: 153-163.

Lawson, K. A., N. R. Dunn, B. A. Roelen, L. M. Zeinstra, A. M. Davis, C. V. Wright, J. P. Korving, and B. L. Hogan. 1999. Bmp4 is required for the generation of primordial germ cells in the mouse embryo. Genes \& development 13: 424-436.

Leatherman, J. L., L. Levin, J. Boero, and T. A. Jongens. 2002. germ cell-less acts to repress transcription during the establishment of the Drosophila germ cell lineage. Current Biology 12: $1681-1685$.

Lee, K. H., S. Lee, B. Kim, S. Chang, S. W. Kim, J. S. Paick, and K. Rhee. 2006. Dazl can bind to dynein motor complex and may play a role in transport of specific mRNAs. Embo $\mathrm{J} 25$ : 4263-4270.

Lee, M. T., A. R. Bonneau, and A. J. Giraldez. 2014. Zygotic Genome Activation During the Maternal-to-Zygotic Transition. Annual review of cell and developmental biology.

Lewis, R. A., T. L. Kress, C. A. Cote, D. Gautreau, M. E. Rokop, and K. L. Mowry. 2004. Conserved and clustered RNA recognition sequences are a critical feature of signals directing RNA localization in Xenopus oocytes. Mech Dev 121: 101-109.

Lin, Y., M. E. Gill, J. Koubova, and D. C. Page. 2008. Germ cell-intrinsic and -extrinsic factors govern meiotic initiation in mouse embryos. Science 322: 1685-1687.

Lize, M., A. Klimke, and M. Dobbelstein. 2011. MicroRNA-449 in cell fate determination. Cell cycle 10: 2874-2882.

Löber, J. 2008. Identifizierung und funktionelle Charakterisierung neuer

RNA-Transportfaktoren in der Xenopus laevis Oozyte. Georg-August-Universität zu Göttingen.

Lund, E., M. D. Sheets, S. B. Imboden, and J. E. Dahlberg. 2011. Limiting Ago protein restricts RNAi and microRNA biogenesis during early development in Xenopus laevis. Genes \& development 25: 1121-1131.

Lund, E., M. Liu, R. S. Hartley, M. D. Sheets, and J. E. Dahlberg. 2009. Deadenylation of maternal mRNAs mediated by miR-427 in Xenopus laevis embryos. Rna 15: 2351-2363.

Ma, K., J. D. Inglis, A. Sharkey, W. A. Bickmore, R. E. Hill, E. J. Prosser, R. M. Speed, E. J. Thomson, M. Jobling, K. Taylor, and et al. 1993. A Y chromosome gene family with RNA-binding protein homology: candidates for the azoospermia factor AZF controlling human spermatogenesis. Cell 75: 1287-1295.

MacArthur, H., M. Bubunenko, D. W. Houston, and M. L. King. 1999. Xcat2 RNA is a translationally sequestered germ plasm component in Xenopus. Mechanisms of development 84: 75-88.

MacArthur, H., D. W. Houston, M. Bubunenko, L. Mosquera, and M. L. King. 2000. DEADSouth is a germ plasm specific DEAD-box RNA helicase in Xenopus related to elF4A. Mechanisms of development 95: 291-295.

Machado, R. J., W. Moore, R. Hames, E. Houliston, P. Chang, M. L. King, and H. R. Woodland. 2005. Xenopus Xpat protein is a major component of germ plasm and may function in its organisation and positioning. Developmental biology 287: 289-300. 
Maegawa, S., M. Yamashita, K. Yasuda, and K. Inoue. 2002. Zebrafish DAZ-like protein controls translation via the sequence 'GUUC'. Genes to cells : devoted to molecular \& cellular mechanisms 7: 971-984.

Mahowald, A. P. 1971a. Polar Granules of Drosophila .3. Continuity of Polar Granules during Life Cycle of Drosophila. J Exp Zool 176: 329-\&.

Mahowald, A. P. 1971b. Polar Granules of Drosophila .4. Cytochemical Studies Showing Loss of Rna from Polar Granules during Early Stages of Embryogenesis. J Exp Zool 176: 345-\&.

Marlow, F. L., and M. C. Mullins. 2008. Bucky ball functions in Balbiani body assembly and animal-vegetal polarity in the oocyte and follicle cell layer in zebrafish. Developmental biology 321: 40-50.

Martinho, R. G., P. S. Kunwar, J. Casanova, and R. Lehmann. 2004. A noncoding RNA is required for the repression of RNApolll-dependent transcription in primordial germ cells. Current biology : CB 14: 159-165.

Matin, A. 2007. What leads from dead-end? Cellular and molecular life sciences : CMLS 64: 1317-1322.

Megosh, H. B., D. N. Cox, C. Campbell, and H. F. Lin. 2006. The role of PIWI and the miRNA machinery in Drosophila germline determination. Current Biology 16: 1884-1894.

Mei, W., Z. Jin, F. Lai, T. Schwend, D. W. Houston, M. L. King, and J. Yang. 2013. Maternal Dead-End1 is required for vegetal cortical microtubule assembly during Xenopus axis specification. Development 140: 2334-2344.

Mickoleit, M., T. U. Banisch, and E. Raz. 2011. Regulation of hub mRNA stability and translation by miR430 and the dead end protein promotes preferential expression in zebrafish primordial germ cells. Developmental dynamics : an official publication of the American Association of Anatomists 240: 695-703.

Mishima, Y., A. J. Giraldez, Y. Takeda, T. Fujiwara, H. Sakamoto, A. F. Schier, and K. Inoue. 2006. Differential regulation of germline mRNAs in soma and germ cells by zebrafish miR-430. Current biology : CB 16: 2135-2142.

Mishima, Y., C. Abreu-Goodger, A. A. Staton, C. Stahlhut, C. Shou, C. Cheng, M. Gerstein, A. J. Enright, and A. J. Giraldez. 2009. Zebrafish miR-1 and miR-133 shape muscle gene expression and regulate sarcomeric actin organization. Genes Dev 23: 619-632.

Mita, K., and M. Yamashita. 2000. Expression of Xenopus Daz-like protein during gametogenesis and embryogenesis. Mechanisms of development 94: 251-255.

Mosquera, L., C. Forristall, Y. Zhou, and M. L. King. 1993. A Messenger-Rna Localized to the Vegetal Cortex of Xenopus Oocytes Encodes a Protein with a Nanos-Like Zinc Finger Domain. Development 117: 377-386.

Mullis, K. B. F. F., S. Scharf, R. Saiki, G. Horn, H. Erlich. 1986. Specific enzymatic amplification of DNA in vitro: the polymerase chain reaction. . Cold Spring Harb Symp Quant Biol.

Newmark, P. A., S. E. Mohr, L. Gong, and R. E. Boswell. 1997. mago nashi mediates the posterior follicle cell-to-oocyte signal to organize axis formation in Drosophila. Development 124: 3197-3207.

Newport, J., and M. Kirschner. 1982. A major developmental transition in early Xenopus embryos: I. characterization and timing of cellular changes at the midblastula stage. Cell 30: 675-686.

Nieuwkoop, P. D., Daudin (ed.) 1994. Normal Table of Xenopus Laevis (Daudin): A Systematical \& Chronological Survey of the Development from the Fertilized Egg Till the End of Metamorphosi ... the Fertilized Egg Till the End of Metamorp) Garland Pub.

Ohinata, Y., H. Ohta, M. Shigeta, K. Yamanaka, T. Wakayama, and M. Saitou. 2009. A signaling principle for the specification of the germ cell lineage in mice. Cell 137: 571-584.

Ohinata, Y., B. Payer, D. O'Carroll, K. Ancelin, Y. Ono, M. Sano, S. C. Barton, T. Obukhanych, M. Nussenzweig, A. Tarakhovsky, M. Saitou, and M. A. Surani. 2005. Blimp1 is a critical determinant of the germ cell lineage in mice. Nature 436: 207-213.

Padmanabhan, K., and J. D. Richter. 2006. Regulated Pumilio-2 binding controls RINGO/Spy mRNA translation and CPEB activation. Genes Dev 20: 199-209.

Paillard, L., and H. B. Osborne. 2003. East of EDEN was a poly(A) tail. Biol Cell 95: 211-219.

Panigrahi, S. K., A. Vasileva, and D. J. Wolgemuth. 2012. Sp1 transcription factor and GATA1 cis-acting elements modulate testis-specific expression of mouse cyclin A1. PloS one 7: e47862. 
Pauli, A., J. L. Rinn, and A. F. Schier. 2011. Non-coding RNAs as regulators of embryogenesis. Nat Rev Genet 12: 136-149.

Reijo, R., J. Seligman, M. B. Dinulos, T. Jaffe, L. G. Brown, C. M. Disteche, and D. C. Page. 1996. Mouse autosomal homolog of DAZ, a candidate male sterility gene in humans, is expressed in male germ cells before and after puberty. Genomics 35: 346-352.

Reijo, R., T. Y. Lee, P. Salo, R. Alagappan, L. G. Brown, M. Rosenberg, S. Rozen, T. Jaffe, D. Straus, O. Hovatta, and et al. 1995a. Diverse spermatogenic defects in humans caused by $\mathrm{Y}$ chromosome deletions encompassing a novel RNA-binding protein gene. Nat Genet 10: 383-393.

Reijo, R., T. Y. Lee, P. Salo, R. Alagappan, L. G. Brown, M. Rosenberg, S. Rozen, T. Jaffe, D. Straus, O. Hovatta, A. Delachapelle, S. Silber, and D. C. Page. 1995b. Diverse Spermatogenic Defects in Humans Caused by Y-Chromosome Deletions Encompassing a Novel Rna-Binding Protein Gene. Nat Genet 10: 383-393.

Reijo, R. A., D. M. Dorfman, R. Slee, A. A. Renshaw, K. R. Loughlin, H. Cooke, and D. C. Page. 2000. DAZ family proteins exist throughout male germ cell development and transit from nucleus to cytoplasm at meiosis in humans and mice. Biology of reproduction 63: 1490-1496.

Reynolds, N., and H. J. Cooke. 2005. Role of the DAZ genes in male fertility. Reproductive biomedicine online 10: 72-80.

Reynolds, N., B. Collier, V. Bingham, N. K. Gray, and H. J. Cooke. 2007. Translation of the synaptonemal complex component Sycp3 is enhanced in vivo by the germ cell specific regulator Dazl. Rna 13: 974-981.

Reynolds, N., B. Collier, K. Maratou, V. Bingham, R. M. Speed, M. Taggart, C. A. Semple, N. K. Gray, and H. J. Cooke. 2005. Dazl binds in vivo to specific transcripts and can regulate the pre-meiotic translation of Mvh in germ cells. Human molecular genetics 14: 3899-3909.

Ruggiu, M., R. Speed, M. Taggart, S. J. McKay, F. Kilanowski, P. Saunders, J. Dorin, and H. J. Cooke. 1997. The mouse Dazla gene encodes a cytoplasmic protein essential for gametogenesis. Nature 389: 73-77.

Rupp, R. A., L. Snider, and H. Weintraub. 1994. Xenopus embryos regulate the nuclear localization of XMyoD. Genes \& development 8: 1311-1323.

Sambrook, J., and Russell, D. W. . 2001. "Molecular Cloning: a laboratory manual." Cold Spring Harbor Laboratory Press Cold Spring Harbor, New York.

Sanger, F., S. Nicklen, and A. R. Coulson. 1992. DNA sequencing with chain-terminating inhibitors. 1977. Biotechnology 24: 104-108.

Santos, A. C., and R. Lehmann. 2004. Germ cell specification and migration in Drosophila and beyond. Current biology : CB 14: R578-589.

Saunders, P. T., J. M. Turner, M. Ruggiu, M. Taggart, P. S. Burgoyne, D. Elliott, and H. J. Cooke. 2003. Absence of mDazl produces a final block on germ cell development at meiosis. Reproduction 126: 589-597.

Schier, A. F. 2007. The maternal-zygotic transition: death and birth of RNAs. Science 316: 406407.

Schrans-Stassen, B. H., P. T. Saunders, H. J. Cooke, and D. G. de Rooij. 2001. Nature of the spermatogenic arrest in Dazl -/- mice. Biology of reproduction 65: 771-776.

Schubiger, G., and W. J. Wood. 1977. Determination during Early Embryogenesis in Drosophila-Melanogaster. Am Zool 17: 565-576.

Seydoux, G., and S. Strome. 1999. Launching the germline in Caenorhabditis elegans: regulation of gene expression in early germ cells. Development 126: 3275-3283.

Sharp P. A., S. B. S. I. 1973. Detection of two restriction endonuclease activities in Haemophilus parainfluenzae using analytical agarose-ethidium bromide electrophoresis. Biochemistry 12:3055-63, 1973. [Cold Spring Harbor Laboratory, Cold Spring Harbor, NY.

Slanchev, K., J. Stebler, M. Goudarzi, V. Cojocaru, G. Weidinger, and E. Raz. 2009. Control of Dead end localization and activity--implications for the function of the protein in antagonizing miRNA function. Mechanisms of development 126: 270-277.

Slee, R., B. Grimes, R. M. Speed, M. Taggart, S. M. Maguire, A. Ross, N. I. McGill, P. T. Saunders, and H. J. Cooke. 1999. A human DAZ transgene confers partial rescue of the mouse Dazl null phenotype. Proceedings of the National Academy of Sciences of the United States of America 96: 8040-8045. 
Smorag, L., X. Xu, W. Engel, and D. V. Pantakani. 2014. The roles of DAZL in RNA biology and development. Wiley interdisciplinary reviews. RNA 5: 527-535.

Song, R., P. Walentek, N. Sponer, A. Klimke, J. S. Lee, G. Dixon, R. Harland, Y. Wan, P. Lishko, M. Lize, M. Kessel, and L. He. 2014. miR-34/449 miRNAs are required for motile ciliogenesis by repressing cp110. Nature 510: 115-120.

Srikantan, S., K. Tominaga, and M. Gorospe. 2012. Functional interplay between RNA-binding protein HuR and microRNAs. Current protein \& peptide science 13: 372-379.

Subramaniam, K., and G. Seydoux. 1999. nos-1 and nos-2, two genes related to Drosophila nanos, regulate primordial germ cell development and survival in Caenorhabditis elegans. Development 126: 4861-4871.

Svoboda, P., and M. Flemr. 2010. The role of miRNAs and endogenous siRNAs in maternal-tozygotic reprogramming and the establishment of pluripotency. EMBO reports 11: 590597.

Tadros, W., and H. D. Lipshitz. 2009. The maternal-to-zygotic transition: a play in two acts. Development 136: 3033-3042.

Takeda, Y., Y. Mishima, T. Fujiwara, H. Sakamoto, and K. Inoue. 2009. DAZL relieves miRNAmediated repression of germline mRNAs by controlling poly $(A)$ tail length in zebrafish. PloS one 4: e7513.

Tanaka, S. S., Y. Toyooka, R. Akasu, Y. Katoh-Fukui, Y. Nakahara, R. Suzuki, M. Yokoyama, and T. Noce. 2000. The mouse homolog of Drosophila Vasa is required for the development of male germ cells. Genes \& development 14: 841-853.

Tang, G. Q., and E. S. Maxwell. 2008. Xenopus microRNA genes are predominantly located within introns and are differentially expressed in adult frog tissues via post-transcriptional regulation. Genome Res 18: 104-112.

Towbin, H., T. Staehelin, and J. Gordon. 1979. Electrophoretic transfer of proteins from polyacrylamide gels to nitrocellulose sheets: procedure and some applications. Proceedings of the National Academy of Sciences of the United States of America 76: 4350-4354.

Treiber, T., N. Treiber, and G. Meister. 2012. Regulation of microRNA biogenesis and function. Thromb Haemost 107: 605-610.

Tsui, S., T. Dai, S. T. Warren, E. C. Salido, and P. H. Yen. 2000a. Association of the mouse infertility factor DAZL1 with actively translating polyribosomes. Biology of reproduction 62: 1655-1660.

Tsui, S., T. Dai, S. Roettger, W. Schempp, E. C. Salido, and P. H. Yen. 2000b. Identification of two novel proteins that interact with germ-cell-specific RNA-binding proteins DAZ and DAZL1. Genomics 65: 266-273.

Tung, J. Y., M. P. Rosen, L. M. Nelson, P. J. Turek, J. S. Witte, D. W. Cramer, M. I. Cedars, and R. A. Pera. 2006. Variants in Deleted in AZoospermia-Like (DAZL) are correlated with reproductive parameters in men and women. Hum Genet 118: 730-740.

Venkatarama, T., F. Lai, X. Luo, Y. Zhou, K. Newman, and M. L. King. 2010. Repression of zygotic gene expression in the Xenopus germline. Development 137: 651-660.

Weidinger, G., J. Stebler, K. Slanchev, K. Dumstrei, C. Wise, R. Lovell-Badge, C. Thisse, B. Thisse, and E. Raz. 2003. dead end, a novel vertebrate germ plasm component, is required for zebrafish primordial germ cell migration and survival. Current biology : CB 13: 1429-1434.

Whitington, P. M., and K. E. Dixon. 1975. Quantitative studies of germ plasm and germ cells during early embryogenesis of Xenopus laevis. Journal of embryology and experimental morphology 33: 57-74.

Wieschaus, E., and D. Sweeton. 1988. Requirements for X-linked zygotic gene activity during cellularization of early Drosophila embryos. Development 104: 483-493.

Winter, J., S. Jung, S. Keller, R. I. Gregory, and S. Diederichs. 2009. Many roads to maturity: microRNA biogenesis pathways and their regulation. Nat Cell Biol 11: 228-234.

Wiszniak, S. E., B. K. Dredge, and K. B. Jensen. 2011. HuB (elavl2) mRNA is restricted to the germ cells by post-transcriptional mechanisms including stabilisation of the message by DAZL. PloS one 6: e20773.

Wylie, C. 1999. Germ cells. Cell 96: 165-174. 
Xu, E. Y., F. L. Moore, and R. A. Pera. 2001. A gene family required for human germ cell development evolved from an ancient meiotic gene conserved in metazoans. Proceedings of the National Academy of Sciences of the United States of America 98: 7414-7419.

Xu, E. Y., D. F. Lee, A. Klebes, P. J. Turek, T. B. Kornberg, and R. A. Reijo Pera. 2003. Human BOULE gene rescues meiotic defects in infertile flies. Human molecular genetics 12: $169-175$.

Xu, X., X. Tan, Q. Lin, B. Schmidt, W. Engel, and D. V. Pantakani. 2013. Mouse Dazl and its novel splice variant functions in translational repression of target mRNAs in embryonic stem cells. Biochimica et biophysica acta 1829: 425-435.

Yamaguchi, T., K. Kataoka, K. Watanabe, and H. Orii. 2014. Restriction of the Xenopus DEADSouth mRNA to the primordial germ cells is ensured by multiple mechanisms. Mechanisms of development 131: 15-23.

Yasuda, G. K., and G. Schubiger. 1992. Temporal regulation in the early embryo: is MBT too good to be true? Trends in genetics : TIG 8: 124-127.

Yen, P. H. 2004. Putative biological functions of the DAZ family. International journal of andrology 27: 125-129.

Yen, P. H., N. N. Chai, and E. C. Salido. 1997. The human DAZ genes, a putative male infertility factor on the $Y$ chromosome, are highly polymorphic in the DAZ repeat regions. Mammalian genome : official journal of the International Mammalian Genome Society 8: 756-759.

Ying, Y., and G. Q. Zhao. 2001. Cooperation of endoderm-derived BMP2 and extraembryonic ectoderm-derived BMP4 in primordial germ cell generation in the mouse. Developmental biology 232: 484-492.

Ying, Y., X. M. Liu, A. Marble, K. A. Lawson, and G. Q. Zhao. 2000. Requirement of Bmp8b for the generation of primordial germ cells in the mouse. Mol Endocrinol 14: 1053-1063.

Yoon, C., K. Kawakami, and N. Hopkins. 1997. Zebrafish vasa homologue RNA is localized to the cleavage planes of 2- and 4-cell-stage embryos and is expressed in the primordial germ cells. Development 124: 3157-3165.

Yoon, Y. J., and K. L. Mowry. 2004. Xenopus Staufen is a component of a ribonucleoprotein complex containing Vg1 RNA and kinesin. Development 131: 3035-3045.

Yoshida, N., T. Tanaka, and M. Yamashita. 1995. Changes in Phosphorylation Activities during Goldfish and Xenopus Oocyte Maturation. Zool Sci 12: 599-606.

Yu, Z., P. Ji, J. Cao, S. Zhu, Y. Li, L. Zheng, X. Chen, and L. Feng. 2009. Dazl promotes germ cell differentiation from embryonic stem cells. Journal of molecular cell biology 1: 93-103.

Yuan, L., J. G. Liu, J. Zhao, E. Brundell, B. Daneholt, and C. Hoog. 2000. The murine SCP3 gene is required for synaptonemal complex assembly, chromosome synapsis, and male fertility. Molecular cell 5: 73-83.

Zeng, M., W. Deng, X. Wang, W. Qiu, Y. Liu, H. Sun, D. Tao, S. Zhang, and Y. Ma. 2008. DAZL binds to the transcripts of several Tssk genes in germ cells. BMB Rep 41: 300-304.

Zeng, M., Y. Lu, X. Liao, D. Li, H. Sun, S. Liang, S. Zhang, Y. Ma, and Z. Yang. 2009. DAZL binds to 3'UTR of Tex19.1 mRNAs and regulates Tex19.1 expression. Molecular biology reports 36: 2399-2403.

Zhang, Q., K. Yaniv, F. Oberman, U. Wolke, A. Git, M. Fromer, W. L. Taylor, D. Meyer, N. Standart, E. Raz, and J. K. Yisraeli. 1999. Vg1 RBP intracellular distribution and evolutionarily conserved expression at multiple stages during development. Mech Dev 88: 101-106.

Zhao, W. M., C. Jiang, T. T. Kroll, and P. W. Huber. 2001. A proline-rich protein binds to the localization element of Xenopus $\mathrm{Vg} 1 \mathrm{mRNA}$ and to ligands involved in actin polymerization. Embo J 20: 2315-2325.

Zhou, Y., and M. L. King. 2004. Sending RNAs into the future: RNA localization and germ cell fate. IUBMB life 56: 19-27. 


\section{APPENDIX}

\subsection{XDazl binds directly to the XDE-LE in ColP experiments}

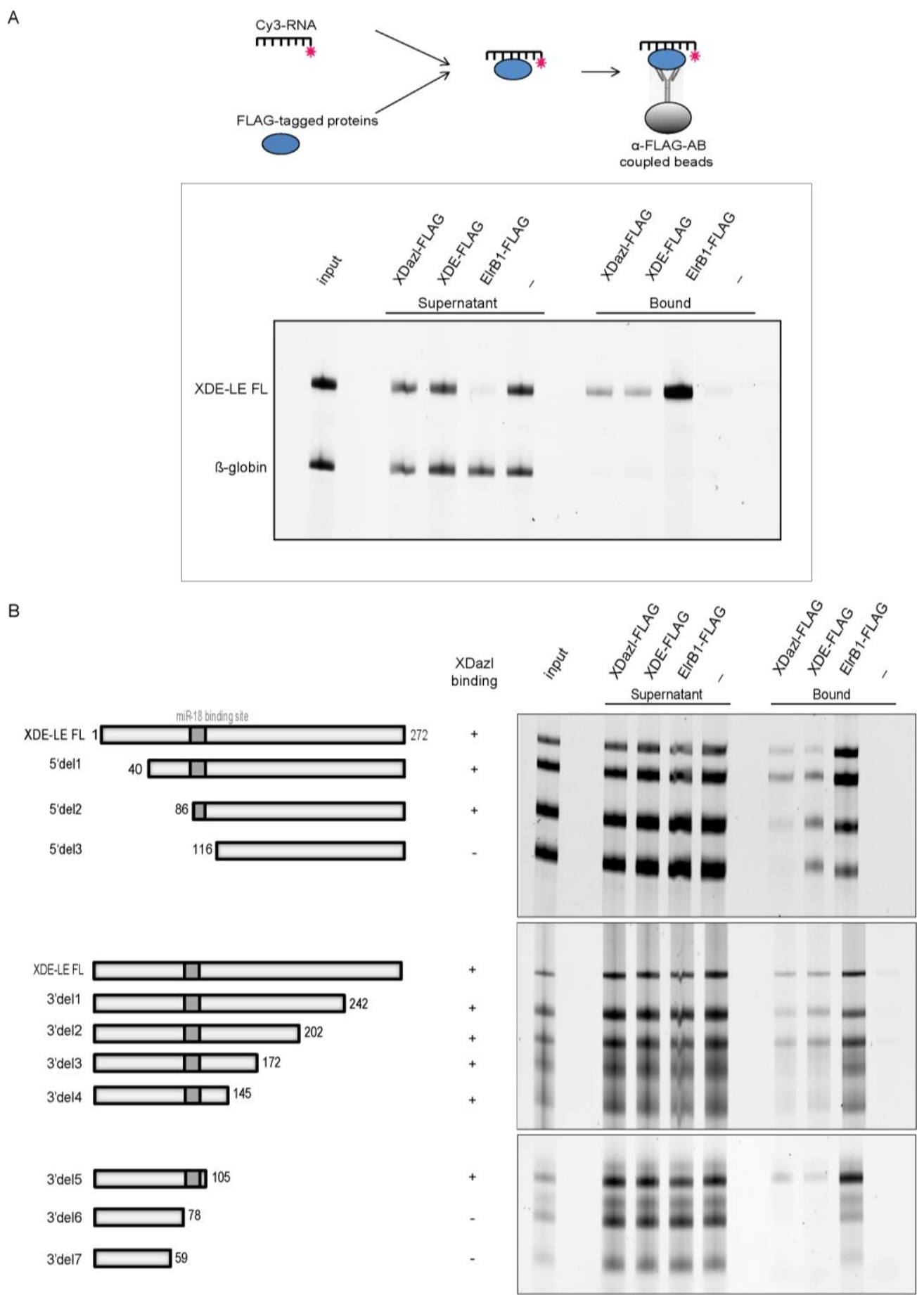

Figure 6.1. XDazl binds directly to the XDE-LE and requires the 5' region of the LE for binding.

$\mathrm{A}+\mathrm{B})$ In vitro transcribed Cy3-labeled RNA of the germline transcripts XDE-LE FL and subfragments were incubated with in vitro translated FLAG-epitope-tagged XDazl, XDE and ElrB1 proteins, immunoprecipitated, isolated and separated on a urea gel.

A) XDazl, XDE and ElrB1 protein bind directly to the XDE-LE FL, whereas the negative control B-globin is not bound.

B) XDazl protein binding requires the XDE-LE 5' region including and surrounding the miR-18 binding site. No XDazl binding is detectable for the incubation with the short subfragments XDE-LE 3'del6 and 3'del7. 


\subsection{XDazl binds directly to the XDazI-LE in ColP experiments}

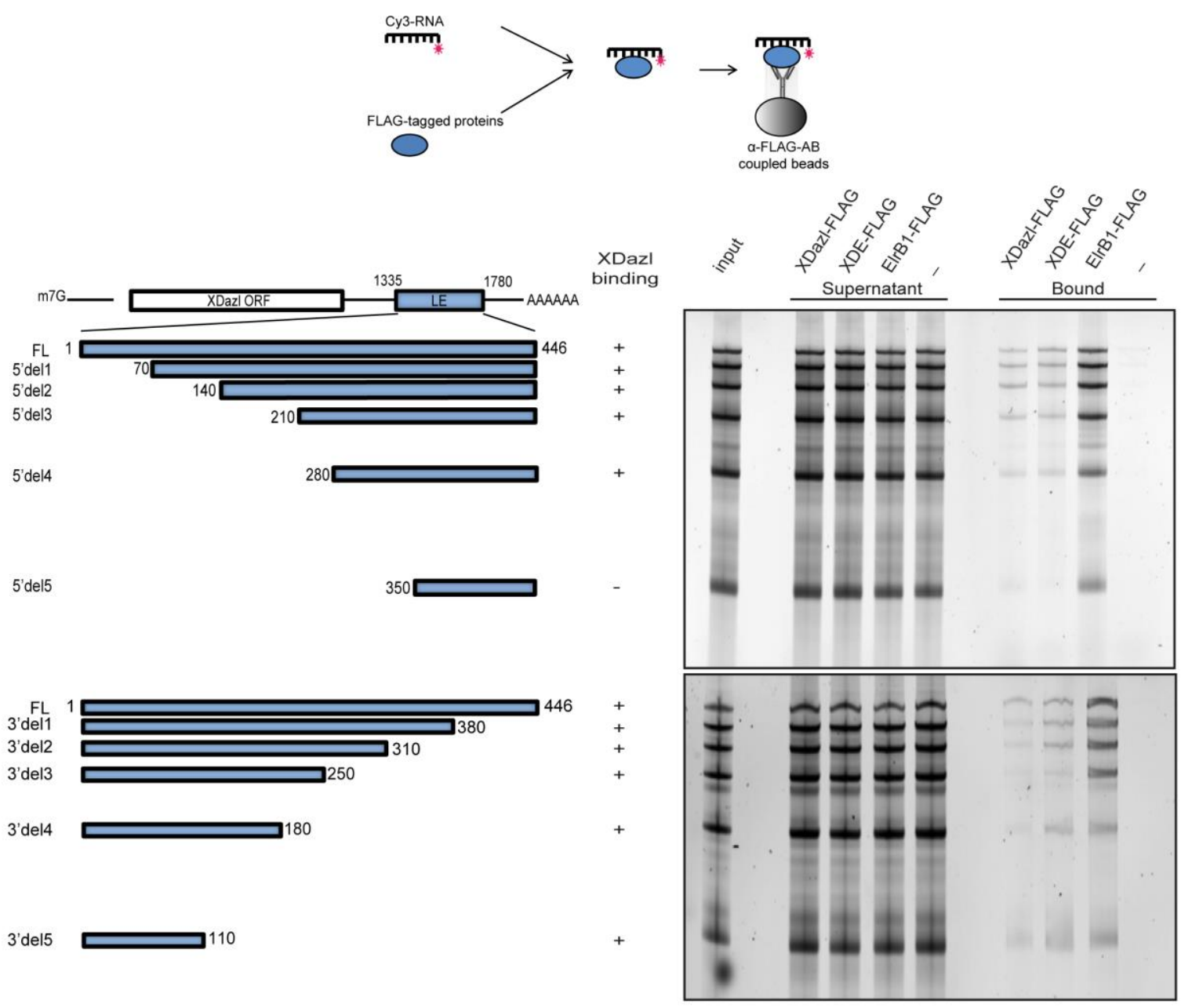

Figure 6.2. XDazl binds directly to the XDazl-LE and has at least two independent binding sites within its LE.

In vitro transcribed Cy3-labeled RNA of the germ cell specific XDazl-LE FL and subfragments were incubated with in vitro translated FLAG-epitope-tagged XDazl, XDE and ElrB1 proteins, immunoprecipitated, isolated and separated on a urea gel. XDazl, XDE and ElrB1 protein bind directly to the XDazl-LE FL and 3' as well 5' subfragments. XDazl and XDE binding is not detectable for the shortest 5' deletion fragment XDazl-LE 5'del5. 


\subsection{Expression of recombinant XDazl protein}

A
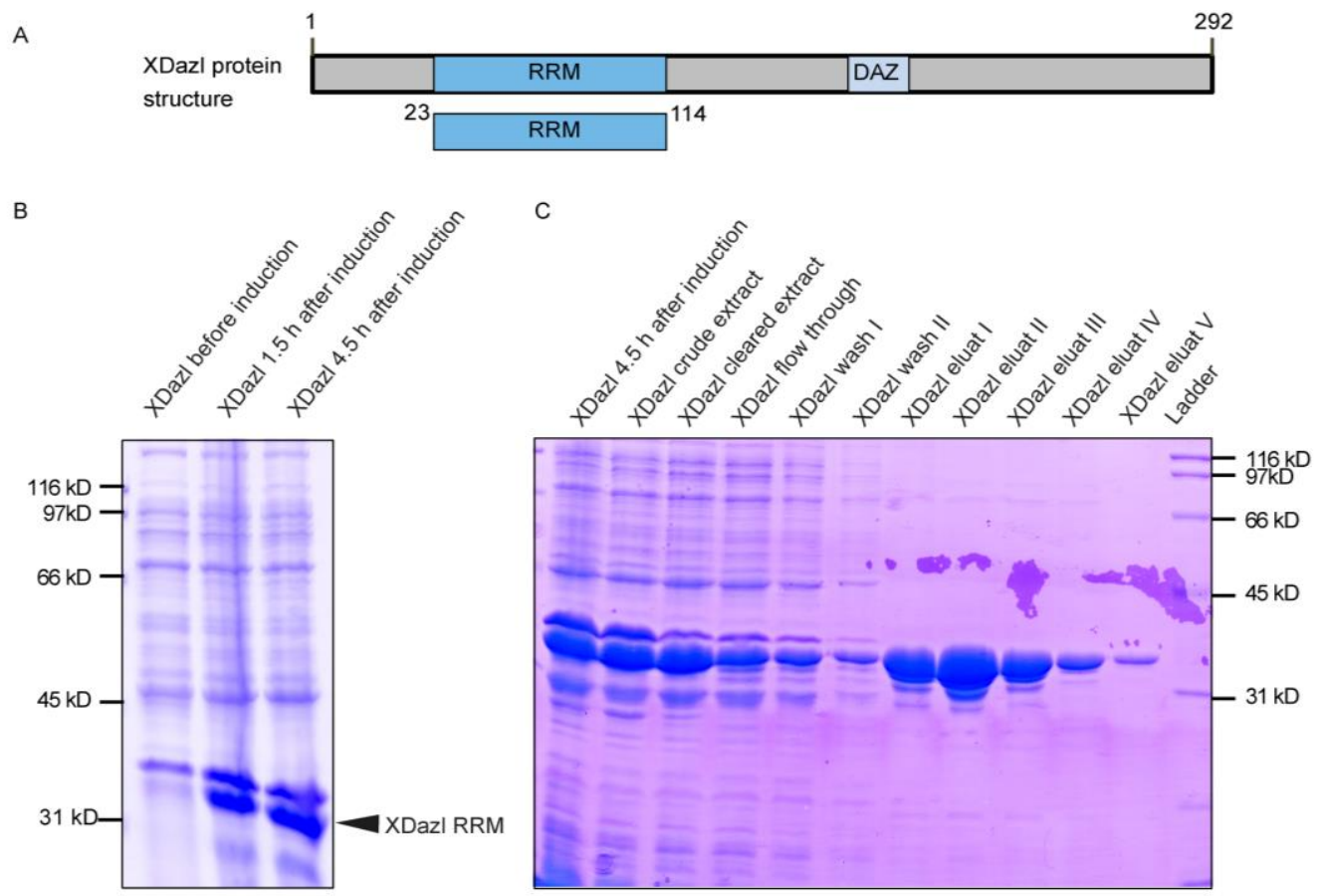

Figure 6.3. Recombinant XDazl RRM protein expression and purification.

A) The 91 amino acid containing XDazl RRM within the XDazl ORF is depicted.

$\mathrm{B}+\mathrm{C})$ Coomassie staining of SDS-PAGEs depicting the recombinant XDazl protein expression before and 4.5 hours after the induction (B) as well as the XDazl RRM protein presence in fractions taken during the different steps of the purification $(C)$.

\subsection{Expression of recombinant XDE protein}

A
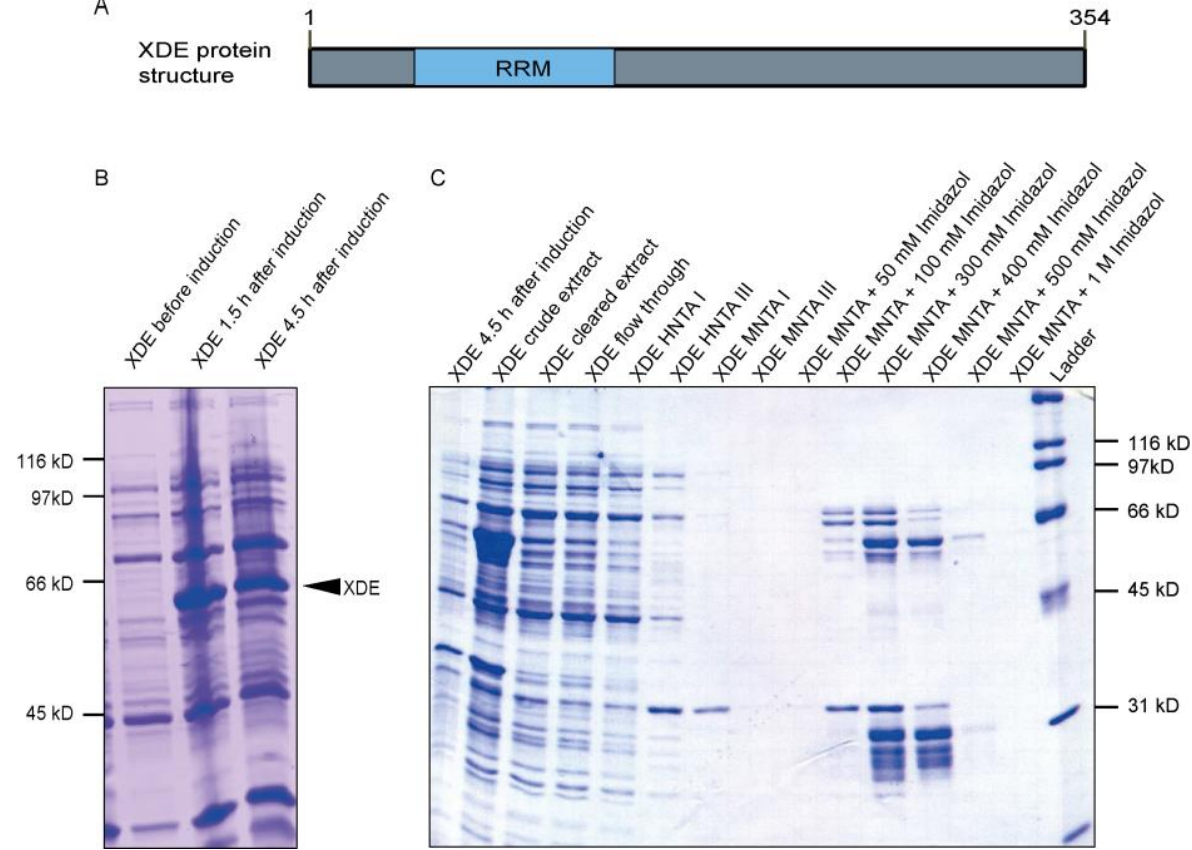

Figure 6.4. Recombinant XDE protein expression and purification.

A) The full length XDE ORF containing one RRM is depicted.

$\mathrm{B}+\mathrm{C})$ Coomassie staining of SDS-PAGEs depicting the recombinant XDE protein expression before and 4.5 hours after the induction $(B)$ as well as the XDE protein presence in fractions taken during the different steps of the purification (C). 


\subsection{Recombinant XDE protein binds to the $5^{\prime}$ region in the XDE-LE}
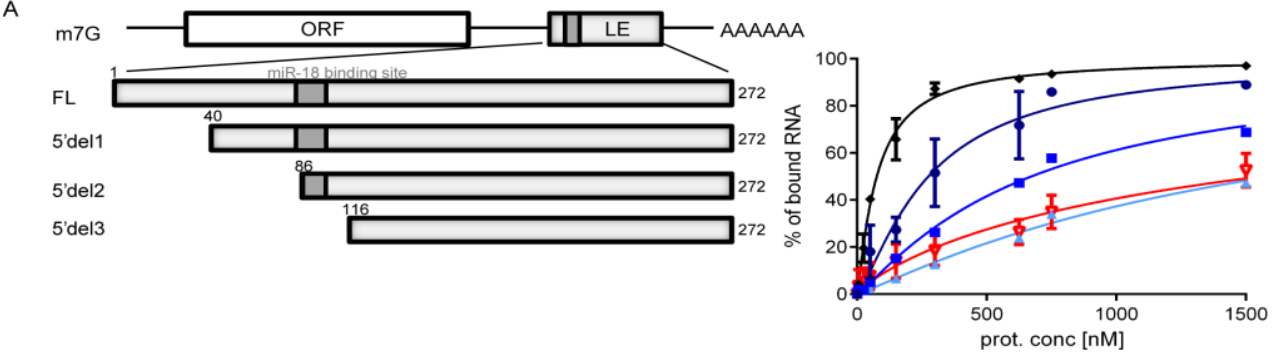

$\rightarrow X D E-L E F L$

- XDE-LE 5'del1

$\because X D E-L E 55^{\prime} d e / 2$

— XDE-LE 5'de/3

$\rightarrow$ B-globin
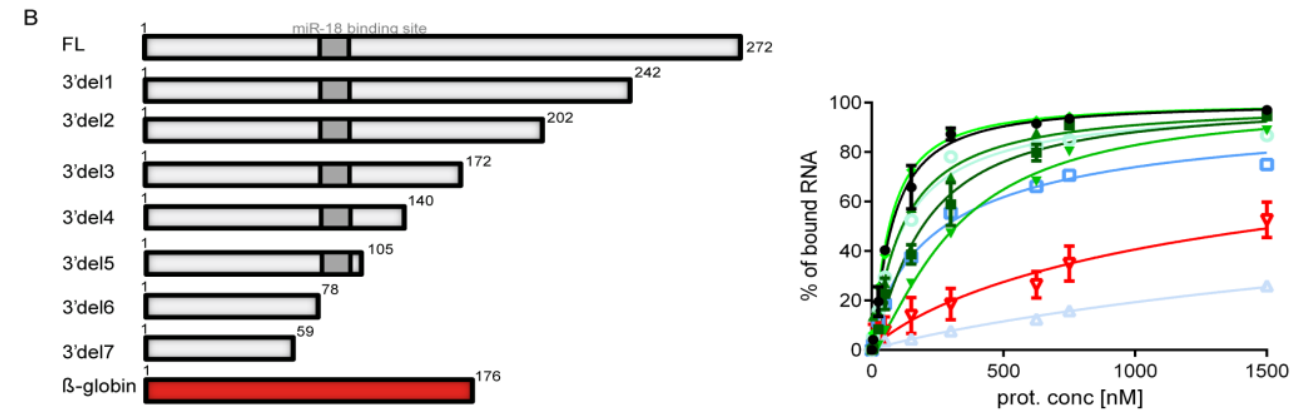

$\rightarrow X D E-L E F L$

$-X D E-L E$ 3'del1

$\mp$ XDE-LE 3'del2

- XDE-LE 3'del3

- XDE-LE 3'del4

$X D E-L E$ 3'del5

- XDE-LE 3'del6

XDE-LE 3'del7

$\rightarrow$ XDE-LE
$\rightarrow$ B-globin

Figure 6.5. Comparative analysis of XDE binding to subfragments of the XDE-LE.

$A+B) C y 3$-labeled $X D E-L E$ deletion fragments were incubated with increasing amounts of XDE protein $(0-1500$ $\mathrm{nM}$ ) and analyzed for RNP complex formation by EMSA.

A) $\mathrm{XDE}$ protein binding to the $X D E-L E$ requires the ${ }^{5}$ ' regions flanking and including the miR-18 binding site. Whereas XDE binding to the $X D E-L E 5$ 'del2 mutant is strongly impaired, the binding is lost upon incubation with $X D E-L E 5^{\prime}$ 'del3 mutant.

B) 3' deletion of the $X D E-L E$ mir-18 binding site is not sufficient to prevent XDE binding. The $X D E-L E 3$ 'del mutants are only impaired for XDE protein binding. Only for the XDE-LE 3'del7 subfragment, in which a region 5 ' to and the miR-18 binding site are deleted, binding is lost.

\subsection{Recombinant ElrB1 binding to the XDE-LE}

A
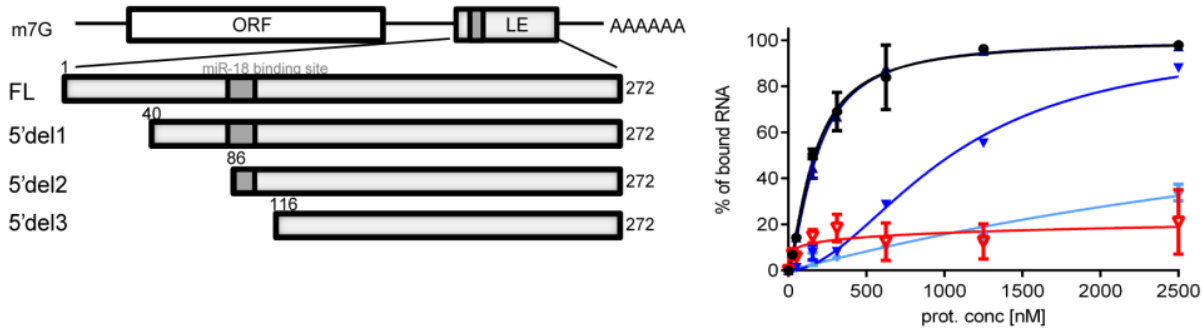

- $X D E-L E F L$

- XDE-LE 5'del 1

- XDE-LE 5'del2

- XDE-LE 5'de/3

$\rightarrow$ B-globin

B
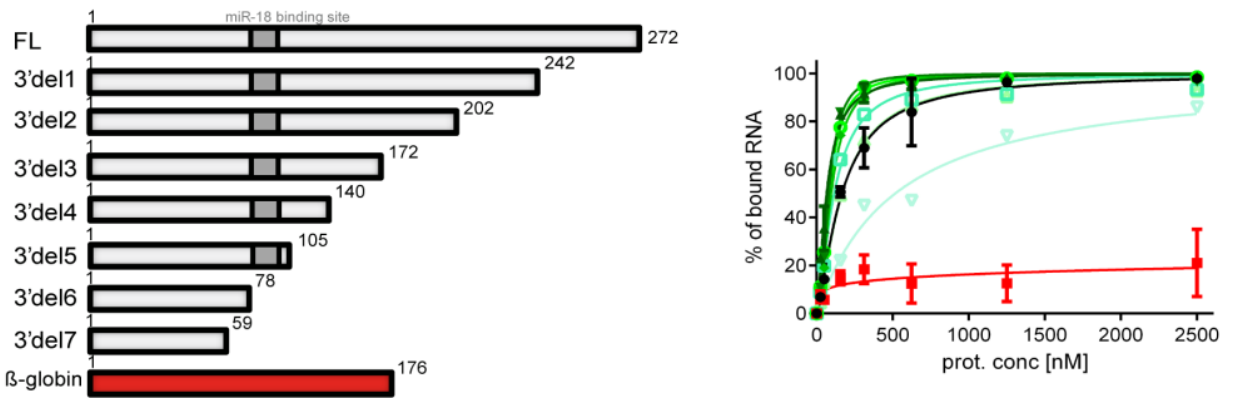

$\rightarrow \quad X D E-L E F L$

- XDE-LE 3'del1

$\rightarrow X D E-L E 3^{\prime} \mathrm{del} / 2$

$\rightarrow X D E-L E$ 3'de/3

- $X D E-L E$ 3'de/4

- XDE-LE 3'de/5

- XDE-LE 3'del6

XDE-LE 3'del7

- B-globin

Figure 6.6. Comparative analysis of ElrB1 binding to subfragments of the XDE-LE.

$A+B) C y 3$-labeled $X D E-L E$ deletion fragments were incubated with increasing amounts of ElrB1 protein $(0-2500$ $\mathrm{nM}$ ) and analyzed for RNP complex formation by EMSA.

A) ElrB1 protein binding to the $X D E-L E$ requires the 5' regions flanking and including the miR-18 binding site. Whereas ElrB1 binding to the $X D E-L E 5$ 'del2 mutant is strongly impaired, the binding is lost upon incubation with $X D E-L E 5^{\prime}$ del3 mutant. B) 3' deletion of the $X D E-L E$ mir-18 binding site is not sufficient to prevent ElrB1 binding. The $X D E-L E 3$ 'del mutants are only impaired for ElrB1 protein binding. Even for the $X D E-L E$ 3'del7 subfragment, in which a region $5^{\prime}$ to and the miR-18 binding site are deleted, reduced binding can be detected. 


\section{7 $\mathrm{K}_{\mathrm{D}}$ values for XDazl, XDE and ElrB1 protein binding to the analyzed RNA}

\section{fragments}

Table 6.1 Summary of all $K_{D}$ values for XDazl, XDE and ElrB1 protein

\begin{tabular}{|c|c|c|c|c|c|c|c|c|c|c|c|c|}
\hline Protein & Fragment & XDE-LE FL & 5 'del1 & 5'del2 & 5 'del3 & 3'del1 & 3'del2 & 3'del3 & 3'del4 & 3'del5 & 3'del6 & 3'del7 \\
\hline XDazl & $K_{D}$ & 221 & 284 & n.d. & n.d. & 1246 & 157 & 215 & 179 & 268 & 933 & n.d. \\
\hline Protein & Fragment & XDazI-LE FL & 5 'del1 & 5'del2 & 5'del3 & 5 'del4 & 5'del5 & 3'del1 & 3'del2 & 3'del3 & 3'del4 & 3'del5 \\
\hline XDazl & $K_{D}$ & 529 & 1151 & 1115 & 1970 & 1608 & 2392 & 1613 & 1263 & n.d. & n.d. & 2176 \\
\hline
\end{tabular}

\begin{tabular}{|llcccccc|}
\hline Protein & Fragment & mut2b & Xpat-LE & Xcat2-LE & XDazl-LE s1 & XDazl-LE s2 & XDazl-LE s3 \\
\hline XDazl & $K_{D}$ & n.d & 274 & n.d. & 1157 & 1158 & 1397 \\
\hline
\end{tabular}

\begin{tabular}{|c|c|c|c|c|c|c|c|c|c|c|c|c|c|}
\hline Protein & Fragment & $\begin{array}{c}\text { XDE-LE } \\
\text { FL } \\
\end{array}$ & $\begin{array}{c}5 \text { 'del } \\
1\end{array}$ & $\begin{array}{c}5 \text { 'del } \\
2 \\
\end{array}$ & $\begin{array}{c}5 \text { 'del } \\
3 \\
\end{array}$ & $\begin{array}{c}\text { 3'del } \\
1 \\
\end{array}$ & $\begin{array}{c}\text { 3'del } \\
2 \\
\end{array}$ & $\begin{array}{c}\text { 3'del } \\
3 \\
\end{array}$ & $\begin{array}{c}\text { 3'del } \\
4 \\
\end{array}$ & $\begin{array}{c}\text { 3'del } \\
5 \\
\end{array}$ & $\begin{array}{c}\text { 3'del } \\
6 \\
\end{array}$ & $\begin{array}{c}\text { 3'del } \\
7 \\
\end{array}$ & $\begin{array}{c}\text { mut2 } \\
\mathrm{b}\end{array}$ \\
\hline$X D E$ & $K_{D}$ & 76 & 275 & 671 & n.d. & 193 & 116 & 321 & 67 & 121 & 276 & n.d. & n.d. \\
\hline
\end{tabular}

\begin{tabular}{|c|c|c|c|c|c|c|c|c|c|c|c|c|c|}
\hline Protein & Fragment & $\begin{array}{c}\text { XDE-LE } \\
\text { FL } \\
\end{array}$ & $\begin{array}{c}5 \text { 'del } \\
1 \\
\end{array}$ & $\begin{array}{c}\text { 5'del } \\
2 \\
\end{array}$ & $\begin{array}{c}\text { 'del } \\
3 \\
\end{array}$ & $\begin{array}{c}\text { 3'del } \\
1\end{array}$ & $\begin{array}{c}\text { 3'del } \\
2\end{array}$ & $\begin{array}{c}\text { 3'del } \\
3\end{array}$ & $\begin{array}{c}3 \text { 'del } \\
4\end{array}$ & $\begin{array}{c}\text { 3'del } \\
5\end{array}$ & $\begin{array}{c}\text { 3'del } \\
6\end{array}$ & $\begin{array}{c}\text { 3'del } \\
7\end{array}$ & $\begin{array}{c}\text { mut2 } \\
\text { b }\end{array}$ \\
\hline ElrB1 & $K_{D}$ & 166 & 182 & 1041 & n.d. & 66 & 80 & 96 & 83 & 115 & 167 & 517 & n.d. \\
\hline
\end{tabular}




\subsection{Summary of RNAs analyzed in NanoString nCounter experiments}

Given are the analyzed RNAs, with all information about the target sequence, Accession number and target site. Furthermore, the primary data, processed data as fold changes, the averaged fold change and the standard deviation of the two experiments are depicted for the performed experiments.

Table 6.2 Summary of RNAs analyzed in NanoString experiments.

Given is the mRNA name, its accession number as well as the target site and the target sequence of the reporter probe. (The nonfunctional $\mathrm{H} 4$ probe is marked in red)

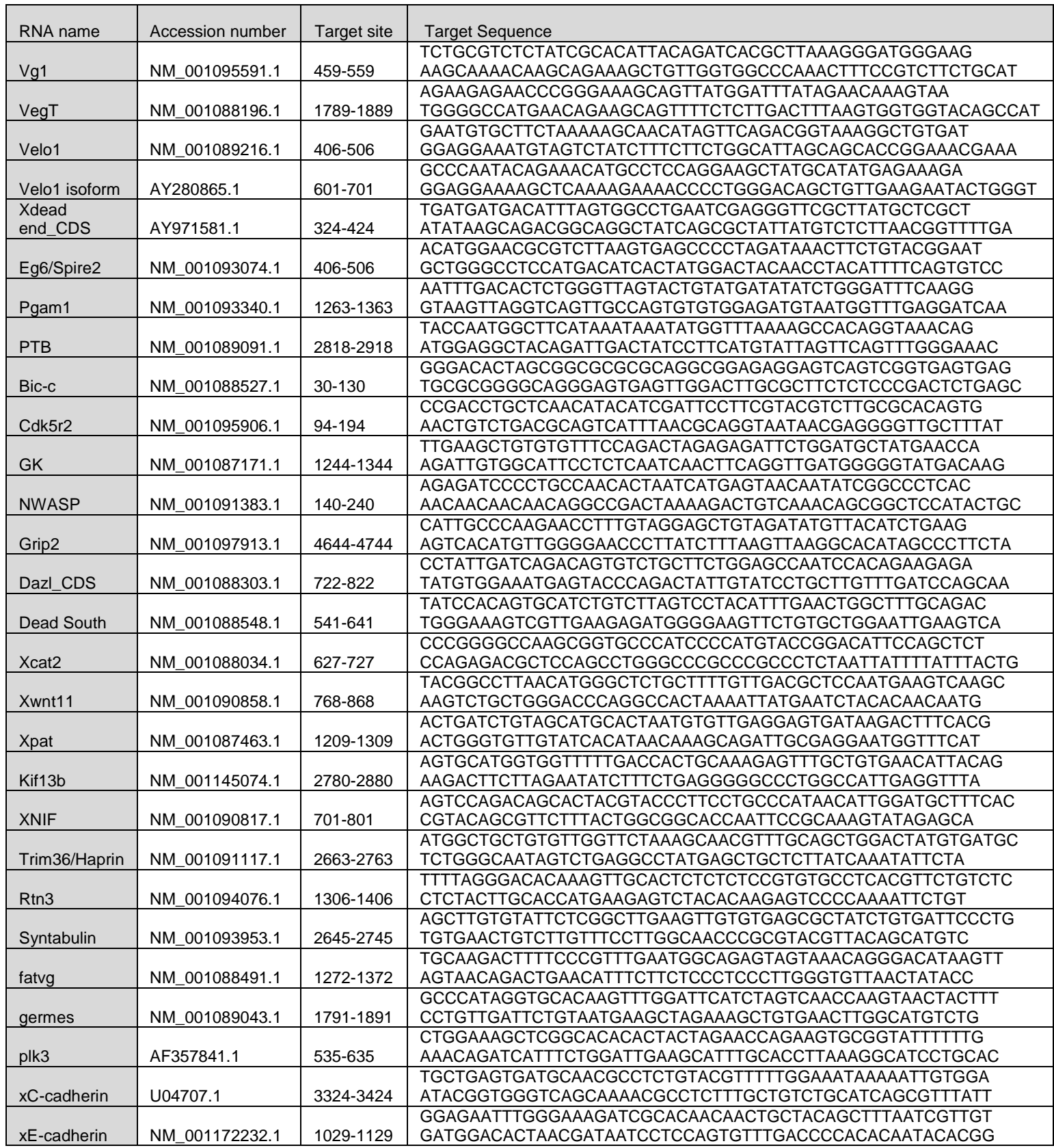




\begin{tabular}{|c|c|c|c|}
\hline Fibronectin 1 & NM 001087801.1 & $3973-4073$ & $\begin{array}{l}\text { CCCCAACTGACTGACATAAAGTATGATGATGTAACTGACACGAGCATTG } \\
\text { ACCTGAGGTGGACGCCCCTTAATTCCTCCAACATTATTGGCTACCGAATCA }\end{array}$ \\
\hline fibronectin rec & NM 001092942.1 & $1089-1189$ & $\begin{array}{l}\text { CAGCGAATTGCAGATGATACATTTAGCCGTCTCCAAAACCTATCCGAGCT } \\
\text { TTCCTTGGTCCGAATTCGTTGGCTGCCCCTCCAGTCAATCTGCCCAGTG }\end{array}$ \\
\hline ArfGAP & NM 001094747.1 & $567-667$ & $\begin{array}{l}\text { AACCTTTTAAAGCGAGGGAGAGATAATGGACAGTACCTGCCTAGAAAGTT } \\
\text { TGTCCTGTCCGAAATAGATGGCACCTTAAAATACTTCACAAAGCCTGATG }\end{array}$ \\
\hline XVLG1 & NM 001088259.1 & $1912-2012$ & $\begin{array}{l}\text { TACCGGTCGCTGTGGTAACACCGGAAAGGCAACATCATTTTTCAATGTT } \\
\text { CAGGATGACCATGTGATTGCTCGTCCCCTTGTGAAAATTCTTACCGATGCT }\end{array}$ \\
\hline Cyclin B2 & NM 001087799.1 & $228-328$ & $\begin{array}{l}\text { CAGTCTTCCAATGCTGTGGCAAAGCCTTCAAAGATGGCAGCAACTAAAGT } \\
\text { GGCAAATGTTAAGACTAAGCATGTACCTGTGAAACCAGTTGTAGCTGAAG }\end{array}$ \\
\hline Cyclin A1 & NM_001094201.1 & $1528-1628$ & $\begin{array}{l}\text { GCTTGCTGTGGGATCAATAGTCTGCAAGCACTTTAGTTAGATGTACTACTAC } \\
\text { AAATCGGAACCCTGTGATCAGAGCTGATTTGCACTGACTAACTGACAC }\end{array}$ \\
\hline Xnr5a/b & NM 001097061.1 & $544-644$ & $\begin{array}{l}\text { CTCCTGGAAAGTCTTTAACCTGACACAGATGCTGCACCGCTACCTCTATCA } \\
\text { TAAAGAAAGAATCTCCAGTGATGAATACACAGATCTACTGGCAACTTCA }\end{array}$ \\
\hline Xnr6b & NM 001095676.1 & $611-711$ & $\begin{array}{l}\text { AGGATATGTCCAAGACAGGCAAGAAGATTAGCTGTACAAATGTGGTTGCA } \\
\text { GAGAGAGTCATTCTGGTGGTATTCGCTAAAGACATACCTTCTGCCAACCT }\end{array}$ \\
\hline Lefty A & NM_001085745.1 & $1371-1471$ & $\begin{array}{l}\text { TGTGTGCCCTACTATCACCATCTTGCCTTACTGACAAGTGTTACTACTATA } \\
\text { GGTGCTGCTGCACATTAAAAAAAGTTGCCTTCCCCAGACTTTATCTGCT }\end{array}$ \\
\hline Lefty $B$ & NM 001088574.1 & $1084-1184$ & $\begin{array}{l}\text { TGGCGGAGTTCCCTAATATGATTGTCGAAAAATGCGGTTGCACAATGGAC } \\
\text { AATATCGCTATCATATGACAGATGGCAGCACTAAGTTCATGCAGATCCGG }\end{array}$ \\
\hline PRDM1 & NM 001087104.1 & $3748-3848$ & $\begin{array}{l}\text { CCTTCCTCTAAACCCATGCCGAAATAACCAGGAAAGGTACAAACTTTAC } \\
\text { AGCTTTAAAAGATCCATGTAGTCCACACGCAGGCCATGGTCTGACTTTATG }\end{array}$ \\
\hline DAZAP2 & NM 001092534.1 & 3296-3396 & $\begin{array}{l}\text { TGGCCACTAATAGGTGGCTATAAGGCATTACGCTAATGACCTCATTTGCA } \\
\text { GAATCTAAAGTTTGAACCTGCACAGGGCTTGCCAATTCACATGTAACCAT }\end{array}$ \\
\hline GS17 & NM_001088064.1 & $71-171$ & $\begin{array}{l}\text { GGATTTCTTGGCCTTGGCTGGCCGTGGAGGTCCTCATTATGCCAGTCCAA } \\
\text { CTTCAAGGCATCCCTCACCAAAGGTTTGGATTCAACACCACCTTCCCTT }\end{array}$ \\
\hline Wnt8 & NM_001088168.1 & $125-225$ & $\begin{array}{l}\text { GTCAGTCAATAACTTTCTGATGACAGGACCCAAGGCATATCTGACATACT } \\
\text { CAGCGAGTGTTGCCGTGGGTGCGCAGAATGGAATTGAGGAGTGTAAATAT }\end{array}$ \\
\hline Chk1 & NM 001088570.1 & $335-435$ & $\begin{array}{l}\text { AGATTTTATGGACATCGAAGGGAAGGCAACATTCAGTACCTCTTTCTGG } \\
\text { AGTATTGTCGAGGTGGTGAGCTCTTTGATCGCATAGAGCCTGATGTTGGAA }\end{array}$ \\
\hline Nek2b & AB019557.1 & $1143-1243$ & $\begin{array}{l}\text { CAAGCCGCCCTAGAAAATGGCATAGGTATGAGAGCCAACAGAAGGCCT } \\
\text { TTGGAGCCCCGGAATGAATGGAGCAAATTCTAATGTTTTTTGTTTCTGTGGG }\end{array}$ \\
\hline CPEB1a & NM 001090603.1 & $1704-1804$ & $\begin{array}{l}\text { GCAGCACTCTATGGAAATCCTGCGCCACCACCGCCCTCTCATGCGTAAT } \\
\text { CAGAAAAGTCGTGACTCCAGCTAAAGACATTGGAACAACATTGGTCCAAAA }\end{array}$ \\
\hline Fyn & NM 001085608.1 & $649-749$ & $\begin{array}{l}\text { ATTGCTCTCTTTTGGGAACCCAAGGGGTACTTATCTAATACGTGAAAGTG } \\
\text { AAACAACCAAAGGTGCCTATTCCTTGTCTATTCGTGACTGGGATGATATG }\end{array}$ \\
\hline Fluc-ORF & X84846.1 & $253-353$ & $\begin{array}{l}\text { TCGAGGTGAACATCACGTACGCGGAATACTTCGAAATGTCCGTTCGGTT } \\
\text { GGCAGAAGCTATGAAACGATATGGGCTGAATACAAATCACAGAATCGTCGT }\end{array}$ \\
\hline Rluc-ORF & HQ425563.1 & $1191-1291$ & $\begin{array}{l}\text { CGCGGCCTCTTCTTATTTATGGCGACATGTTGTGCCACATATTGAGCCAG } \\
\text { TAGCGCGGTGTATTATACCAGACCTTATTGGTATGGGCAAATCAGGCAAA }\end{array}$ \\
\hline lacZ-fragment & JN201970.1 & $6190-6290$ & $\begin{array}{l}\text { GTGCCGGAAAGCTGGCTGGAGTGCGATCTTCCTGAGGCCGATACTGTCG } \\
\text { TCGTCCCCTCAAACTGGCAGATGCACGGTTACGATGCGCCCATCTACACCA }\end{array}$ \\
\hline GFP-ORF & Transpor_001.1 & $281-381$ & $\begin{array}{l}\text { GGAGAGGACCATCTTCTTCAAGGACGACGGGAACTACAAGACACGTGC } \\
\text { TGAAGTCAAGTTTGAGGGAGACACCCTCGTCAACAGGATCGAGCTTAAGGGA }\end{array}$ \\
\hline b-actin & NM 001088953.1 & $1179-1279$ & $\begin{array}{l}\text { ATGCTTCTAAAGGACAGACCCTTTCAACATGAACAAATGTACCTGTGCAGG } \\
\text { AAGATCACATTGGCATGGCTTTACTCTTTTGTTGGCGCTTGGCTCAGAA }\end{array}$ \\
\hline GapDH & NM 001087098.1 & $773-873$ & $\begin{array}{l}\text { ACCTGCCGCCTGCAGAAGCCGGCCAAGTACGATGACATCAAGGCCGCCA } \\
\text { TTAAGACTGCATCAGAGGGCCCAATGAAGGGATCCTGGGATACACACAAG }\end{array}$ \\
\hline ODC & NM 001086698.1 & $855-955$ & $\begin{array}{l}\text { GGATATAATTGGTGTGAGTTTCCATGTTGGCAGTGGCTGCACTGATCCAC } \\
\text { AGACTTATGTACAAGCTGTCTCAGATGCACGATGTGTCTTTGACATGGGG }\end{array}$ \\
\hline laminB1 & NM 001086584.1 & $2140-2240$ & $\begin{array}{l}\text { CAGGTCCTGGAGTGAAATATCTCTGCGATTGCTTCTCCACTTGTCTCAC } \\
\text { TTCAGACACCTTACCCCGAGGTGCTTCACCTTCTCCATTTCCCTCGCTTCC }\end{array}$ \\
\hline G6PDH & NM 001086550.1 & $862-962$ & $\begin{array}{l}\text { GTGGAGGATACTTTGACGAATTTGGCATCATCCGGGATGTCATGCAAAA } \\
\text { TCACTTGCTCCAAATGATGTGTTTGATGGCTATGGAGAAGCCGGTCTCCAC }\end{array}$ \\
\hline $\mathrm{H} 4$ & NM 001094457.1 & $655-755$ & $\begin{array}{l}\text { CCACGCCCCTTCTCCCCATAAAATCAGTTACAGGCTCTCGGGCTCTTTTG } \\
\text { CTCTTGCCGGATGGAAATTACTGTTGCTGCTCAGCGTCTCACAGAACTCC }\end{array}$ \\
\hline HPRT & NM 001096766.1 & $233-333$ & $\begin{array}{l}\text { CTGTGTGTCCTGAAGGGTGGCTATAAGTTCTTTGCTGATCTACTTGACT } \\
\text { ACATTAAAGCACTTAACCGCAACAGTGACAAGTCTATCCCTATGACAGTAG }\end{array}$ \\
\hline $\begin{array}{l}\text { Xdead } \\
\text { end UTR }\end{array}$ & AY971581.1 & $1440-1540$ & $\begin{array}{l}\text { TTCACCTGGAAAGGCAGTTTTTCATAAAGGGCTTTCCTGGTGAAAACTG } \\
\text { TCTGTCCAGAACTTTGAAGTGAGGCGATGAGCTGAAACGTCATCTGCCTGC }\end{array}$ \\
\hline Dazl UTR & NM 001088303.1 & $1255-1355$ & $\begin{array}{l}\text { ATGGGTCTGAGTACTGCTGGGCATATAGACATGTAGTCGTAGGCAGTGG } \\
\text { AGGGGCCTCCAGTCCAGAAGAAGGAATAATATCACTGAGGGGCTTGAGAAA }\end{array}$ \\
\hline
\end{tabular}




\subsubsection{Primary data from NanoString nCounter experiments - ectopic XDazI}

(Figure 3.3)

Primary NanoString data analyzing the influence of ectopic XDazl expression on the stability of germ cell specific mRNAs for two independent experiments is shown in Table 6.3.

Table 6.3 Primary data of RNAs analyzed in NanoString experiments - ectopic XDazl

\begin{tabular}{|c|c|c|c|c|}
\hline & \multicolumn{2}{|c|}{ experiment 1. stage 10.5} & \multicolumn{2}{|c|}{ experiment 2. stage 10.5} \\
\hline RNA name & XDE-LE & XDE-LE + XDazl & XDE-LE & XDE-LE + XDazl \\
\hline ArfGAP & 1298 & 1279 & 414 & 119 \\
\hline Bic-c & 292 & 230 & 463 & 389 \\
\hline CPEB1a & 122 & 205 & 725 & 1115 \\
\hline Cdk5r2 & 62 & 52 & 275 & 393 \\
\hline Chk1 & 437 & 530 & 647 & 744 \\
\hline Cyclin A1 & 435 & 558 & 3298 & 4615 \\
\hline Cyclin B2 & 2756 & 2973 & 6630 & 5294 \\
\hline DAZAP2 & 2244 & 2014 & 1031 & 537 \\
\hline Dazl_CDS & 416 & 32492 & 840 & 13754 \\
\hline Dazl_UTR & 326 & 540 & 498 & 380 \\
\hline Dead South & 1002 & 1527 & 1576 & 1483 \\
\hline Eg6/Spire2 & 214 & 306 & 571 & 648 \\
\hline Fibronectin 1 & 14079 & 12338 & 5403 & 2835 \\
\hline Fluc-ORF & 5 & 1 & 4 & 2 \\
\hline Fyn & 155 & 139 & 136 & 117 \\
\hline G6PDH & 676 & 451 & 1039 & 698 \\
\hline GFP-ORF & 7431 & 13639 & 22653 & 8504 \\
\hline GK & 212 & 133 & 214 & 168 \\
\hline GS17 & 2487 & 1888 & 7302 & 4357 \\
\hline GapDH & 1101 & 704 & 655 & 658 \\
\hline Grip2 & 442 & 645 & 1161 & 1117 \\
\hline $\mathrm{H} 4$ & 92 & 44 & 35 & 25 \\
\hline HPRT & 958 & 836 & 473 & 725 \\
\hline Kif13b & 455 & 425 & 640 & 445 \\
\hline Lefty A & 224 & 154 & 379 & 818 \\
\hline Lefty B & 199 & 155 & 398 & 446 \\
\hline NWASP & 871 & 728 & 704 & 429 \\
\hline Nek2b & 306 & 292 & 360 & 409 \\
\hline ODC & 51066 & 42837 & 26576 & 13474 \\
\hline PRDM1 & 1088 & 550 & 765 & 414 \\
\hline PTB & 2279 & 2346 & 3979 & 3057 \\
\hline Pgam1 & 249 & 289 & 349 & 584 \\
\hline Rluc-ORF & 1236 & 1261 & 6231 & 717 \\
\hline Rtn3 & 632 & 583 & 477 & 317 \\
\hline Syntabulin & 25 & 49 & 40 & 74 \\
\hline Trim36/Haprin & 104 & 91 & 77 & 95 \\
\hline VegT & 3290 & 3629 & 12250 & 9727 \\
\hline Velo1 & 236 & 300 & 1299 & 1546 \\
\hline Velo1 isoform & 86 & 137 & 375 & 391 \\
\hline Vg1 & 381 & 1000 & 3127 & 3609 \\
\hline Wnt8 & 1589 & 1754 & 2600 & 1141 \\
\hline XNIF & 2081 & 1801 & 3036 & 2115 \\
\hline XVLG1 & 1890 & 1806 & 1637 & 1049 \\
\hline Xcat2 & 52 & 49 & 158 & 133 \\
\hline Xdead end_CDS & 182 & 470 & 576 & 840 \\
\hline Xdead end_UTR & 36 & 73 & 47 & 83 \\
\hline Xnr5a/b & 16 & 19 & 67 & 73 \\
\hline Xnr6b & 15 & 14 & 25 & 22 \\
\hline Xpat & 1040 & 1618 & 1794 & 1459 \\
\hline Xwnt11 & 4271 & 3842 & 4110 & 1908 \\
\hline b-actin & 64223 & 54876 & 25011 & 15515 \\
\hline fatvg & 413 & 432 & 899 & 763 \\
\hline fibronectin receptor & 22 & 32 & 134 & 92 \\
\hline germes & 60 & 89 & 187 & 192 \\
\hline lacZ-fragment & 40 & 15 & 9 & 8 \\
\hline laminB1 & 8718 & 6843 & 5195 & 2718 \\
\hline plk3 & 2322 & 1851 & 2579 & 2267 \\
\hline$x C$-cadherin & 7128 & 5247 & 4673 & 2513 \\
\hline xE-cadherin & 5094 & 5821 & 2622 & 631 \\
\hline NEG_A & 22 & 7 & 4 & 5 \\
\hline NEG_B & 12 & 10 & 3 & 3 \\
\hline NEG_C & 7 & 3 & 5 & 7 \\
\hline NEG_D & 24 & 15 & 9 & 2 \\
\hline NEG_E & 26 & 4 & 6 & 4 \\
\hline NEG_F & 27 & 7 & 12 & 5 \\
\hline NEG_G & 11 & 2 & 4 & 1 \\
\hline NEG_H & 16 & 8 & 6 & 4 \\
\hline POS_A & 89671 & 29207 & 18233 & 11033 \\
\hline POS_B & 26922 & 9167 & 7259 & 4412 \\
\hline POS_C & 6883 & 2555 & 2174 & 1290 \\
\hline POS_D & 1411 & 535 & 350 & 247 \\
\hline POS_E & 535 & 145 & 112 & 78 \\
\hline POS F & 153 & 69 & 53 & 25 \\
\hline
\end{tabular}




\subsubsection{Processed data from NanoString nCounter analysis - ectopic XDazl}

(Figure 3.3)

Fold changes over uninjected embryos for experiment 1 and experiment 2 were calculated and are shown in Table 6.4. Averaged fold changes over uninjected embryos and standard deviation are shown in Table 6.5.

Table 6.4 Fold changes over uninjected embryos of two independent experiments

\begin{tabular}{|c|c|c|c|c|}
\hline & \multicolumn{2}{|c|}{ experiment 1 , stage 10.5} & \multicolumn{2}{|c|}{ experiment 2 , stage 10.5} \\
\hline RNA name & XDE-LE & XDE-LE + XDazI & XDE-LE & XDE-LE + XDazI \\
\hline ArfGAP & 1.0 & 1.3 & 1.0 & 0.5 \\
\hline Bic-c & 1.0 & 1.1 & 1.0 & 1.4 \\
\hline Cdk5r2 & 1.0 & 1.7 & 1.0 & 2.5 \\
\hline Chk1 & 1.0 & 1.7 & 1.0 & 2.0 \\
\hline DAZAP2 & 1.0 & 1.2 & 1.0 & 0.9 \\
\hline Dazl_CDS & 1.0 & 112.5 & 1.0 & 28.4 \\
\hline Dazl_UTR & 1.0 & 2.4 & 1.0 & 1.3 \\
\hline Dead South & 1.0 & 2.1 & 1.0 & 1.6 \\
\hline Eg6/Spire2 & 1.0 & 2.1 & 1.0 & 2.0 \\
\hline GFP-ORF & 1.0 & 2.4 & 1.0 & 0.6 \\
\hline GK & 1.0 & 0.9 & 1.0 & 1.4 \\
\hline GS17 & 1.0 & 1.0 & 1.0 & 1.0 \\
\hline GapDH & 1.0 & 0.9 & 1.0 & 1.7 \\
\hline Grip2 & 1.0 & 2.0 & 1.0 & 1.7 \\
\hline $\mathrm{H} 4$ & 1.0 & 0.6 & 1.0 & 1.3 \\
\hline HPRT & 1.0 & 1.2 & 1.0 & 2.7 \\
\hline Kif13b & 1.0 & 1.3 & 1.0 & 1.2 \\
\hline Lefty A & 1.0 & 1.0 & 1.0 & 3.8 \\
\hline Lefty B & 1.0 & 1.1 & 1.0 & 1.9 \\
\hline NWASP & 1.0 & 1.1 & 1.0 & 1.0 \\
\hline Syntabulin & 1.0 & 44.7 & 1.0 & 4.1 \\
\hline Trim36/Haprin & 1.0 & 1.4 & 1.0 & 2.3 \\
\hline VegT & 1.0 & 1.5 & 1.0 & 1.4 \\
\hline Velo1 & 1.0 & 1.9 & 1.0 & 2.0 \\
\hline Velo1 isoform & 1.0 & 3.1 & 1.0 & 1.8 \\
\hline $\operatorname{Vg} 1$ & 1.0 & 3.8 & 1.0 & 2.0 \\
\hline Wnt8 & 1.0 & 1.5 & 1.0 & 0.8 \\
\hline XNIF & 1.0 & 1.2 & 1.0 & 1.2 \\
\hline XVLG1 & 1.0 & 1.3 & 1.0 & 1.1 \\
\hline Xcat2 & 1.0 & 2.4 & 1.0 & 1.5 \\
\hline Xdead end_CDS & 1.0 & 4.1 & 1.0 & 2.5 \\
\hline Xdead end_UTR & 1.0 & 29.1 & 1.0 & 3.7 \\
\hline Xnr5a/b & 1.0 & 4.8 & 1.0 & 2.0 \\
\hline Xnr6b & 1.0 & 1.3 & 1.0 & 1.9 \\
\hline Xpat & 1.0 & 2.1 & 1.0 & 1.4 \\
\hline Xwnt11 & 1.0 & 1.2 & 1.0 & 0.8 \\
\hline b-actin & 1.0 & 1.1 & 1.0 & 1.1 \\
\hline fatvg & 1.0 & 1.5 & 1.0 & 1.5 \\
\hline fibronectin receptor & 1.0 & 22.1 & 1.0 & 1.2 \\
\hline germes & 1.0 & 3.7 & 1.0 & 1.8 \\
\hline lacZ-fragment & 1.0 & 0.2 & 1.0 & 0.9 \\
\hline laminB1 & 1.0 & 1.0 & 1.0 & 0.9 \\
\hline
\end{tabular}


Table 6.5 Averaged fold changes over uninjected embryos of two independent experiments and calculated standard deviation

\begin{tabular}{|c|c|c|}
\hline & \multicolumn{2}{|c|}{ fold change exp. $1+2$} \\
\hline RNA name & XDE-LE & $\begin{array}{c}\text { XDE-LE + } \\
\text { XDazl }\end{array}$ \\
\hline ArfGAP & 1.0 & 0.9 \\
\hline Bic-c & 1.0 & 1.3 \\
\hline CPEB1a & 1.0 & 2.8 \\
\hline Cdk5r2 & 1.0 & 2.1 \\
\hline Chk1 & 1.0 & 1.9 \\
\hline Cyclin A1 & 1.0 & 2.1 \\
\hline Cyclin B2 & 1.0 & 1.4 \\
\hline DAZAP2 & 1.0 & 1.1 \\
\hline Dazl_CDS & 1.0 & 70.5 \\
\hline Dazl_UTR & 1.0 & 1.9 \\
\hline Dead South & 1.0 & 1.9 \\
\hline Eg6/Spire2 & 1.0 & 2.1 \\
\hline Fibronectin 1 & 1.0 & 1.1 \\
\hline Fluc-ORF & 1.0 & 1.5 \\
\hline Fyn & 1.0 & 1.4 \\
\hline G6PDH & 1.0 & 1.1 \\
\hline GFP-ORF & 1.0 & 1.5 \\
\hline GK & 1.0 & 1.2 \\
\hline GS17 & 1.0 & 1.0 \\
\hline GapDH & 1.0 & 1.3 \\
\hline Grip2 & 1.0 & 1.9 \\
\hline $\mathrm{H} 4$ & 1.0 & 1.0 \\
\hline HPRT & 1.0 & 2.0 \\
\hline Kif13b & 1.0 & 1.3 \\
\hline Lefty $A$ & 1.0 & 2.4 \\
\hline Lefty B & 1.0 & 1.5 \\
\hline NWASP & 1.0 & 1.1 \\
\hline Nek2b & 1.0 & 1.7 \\
\hline ODC & 1.0 & 1.0 \\
\hline PRDM1 & 1.0 & 0.8 \\
\hline PTB & 1.0 & 1.4 \\
\hline Pgam1 & 1.0 & 2.3 \\
\hline Rluc-ORF & 1.0 & 0.8 \\
\hline Rtn3 & 1.0 & 1.2 \\
\hline Syntabulin & 1.0 & 24.4 \\
\hline Trim36/Haprin & 1.0 & 1.9 \\
\hline VegT & 1.0 & 1.5 \\
\hline Velo1 & 1.0 & 2.0 \\
\hline Velo1 isoform & 1.0 & 2.5 \\
\hline $\operatorname{Vg} 1$ & 1.0 & 2.9 \\
\hline Wnt8 & 1.0 & 1.2 \\
\hline XNIF & 1.0 & 1.2 \\
\hline XVLG1 & 1.0 & 1.2 \\
\hline Xcat2 & 1.0 & 2.0 \\
\hline Xdead end_CDS & 1.0 & 3.3 \\
\hline Xdead end_UTR & 1.0 & 16.4 \\
\hline Xnr5a/b & 1.0 & 3.4 \\
\hline Xnr6b & 1.0 & 1.6 \\
\hline Xpat & 1.0 & 1.8 \\
\hline Xwnt11 & 1.0 & 1.0 \\
\hline b-actin & 1.0 & 1.1 \\
\hline fatvg & 1.0 & 1.5 \\
\hline fibronectin receptor & 1.0 & 11.7 \\
\hline germes & 1.0 & 2.8 \\
\hline lacZ-fragment & 1.0 & 0.6 \\
\hline laminB1 & 1.0 & 1.0 \\
\hline plk3 & 1.0 & 1.3 \\
\hline xC-cadherin & 1.0 & 1.0 \\
\hline xE-cadherin & 1.0 & 1.0 \\
\hline
\end{tabular}

\begin{tabular}{|c|c|c|}
\hline & \multicolumn{2}{|c|}{ std dev exp. $1+2$} \\
\hline RNA name & XDE-LE & $\begin{array}{c}\text { XDE-LE + } \\
\text { XDazl }\end{array}$ \\
\hline ArfGAP & 0.0 & 0.6 \\
\hline Bic-c & 0.0 & 0.2 \\
\hline CPEB1a & 0.0 & 0.1 \\
\hline Cdk5r2 & 0.0 & 0.6 \\
\hline Chk1 & 0.0 & 0.2 \\
\hline Cyclin A1 & 0.0 & 0.4 \\
\hline Cyclin B2 & 0.0 & 0.0 \\
\hline DAZAP2 & 0.0 & 0.2 \\
\hline Dazl_CDS & 0.0 & 59.5 \\
\hline Dazl_UTR & 0.0 & 0.8 \\
\hline Dead South & 0.0 & 0.4 \\
\hline Eg6/Spire2 & 0.0 & 0.1 \\
\hline Fibronectin 1 & 0.0 & 0.2 \\
\hline Fluc-ORF & 0.0 & 0.3 \\
\hline Fyn & 0.0 & 0.1 \\
\hline G6PDH & 0.0 & 0.2 \\
\hline GFP-ORF & 0.0 & 1.3 \\
\hline GK & 0.0 & 0.4 \\
\hline GS17 & 0.0 & 0.0 \\
\hline GapDH & 0.0 & 0.6 \\
\hline Grip2 & 0.0 & 0.2 \\
\hline $\mathrm{H} 4$ & 0.0 & 0.5 \\
\hline HPRT & 0.0 & 1.1 \\
\hline Kif13b & 0.0 & 0.1 \\
\hline Lefty A & 0.0 & 2.0 \\
\hline Lefty B & 0.0 & 0.6 \\
\hline NWASP & 0.0 & 0.1 \\
\hline Nek2b & 0.0 & 0.5 \\
\hline ODC & 0.0 & 0.1 \\
\hline PRDM1 & 0.0 & 0.1 \\
\hline РTB & 0.0 & 0.1 \\
\hline Pgam1 & 0.0 & 0.8 \\
\hline Rluc-ORF & 0.0 & 0.8 \\
\hline Rtn3 & 0.0 & 0.1 \\
\hline Syntabulin & 0.0 & 28.7 \\
\hline Trim36/Haprin & 0.0 & 0.6 \\
\hline VegT & 0.0 & 0.1 \\
\hline Velo1 & 0.0 & 0.1 \\
\hline Velo1 isoform & 0.0 & 0.9 \\
\hline Vg1 & 0.0 & 1.3 \\
\hline Wnt8 & 0.0 & 0.5 \\
\hline XNIF & 0.0 & 0.0 \\
\hline XVLG1 & 0.0 & 0.1 \\
\hline Xcat2 & 0.0 & 0.6 \\
\hline Xdead end_CDS & 0.0 & 1.1 \\
\hline Xdead end_UTR & 0.0 & 18.0 \\
\hline Xnr5a/b & 0.0 & 2.0 \\
\hline Xnr6b & 0.0 & 0.4 \\
\hline Xpat & 0.0 & 0.5 \\
\hline Xwnt11 & 0.0 & 0.3 \\
\hline b-actin & 0.0 & 0.0 \\
\hline fatvg & 0.0 & 0.0 \\
\hline fibronectin receptor & 0.0 & 14.8 \\
\hline germes & 0.0 & 1.3 \\
\hline lacZ-fragment & 0.0 & 0.5 \\
\hline laminB1 & 0.0 & 0.1 \\
\hline plk3 & 0.0 & 0.3 \\
\hline $\mathrm{xC}$-cadherin & 0.0 & 0.1 \\
\hline xE-cadherin & 0.0 & 0.8 \\
\hline
\end{tabular}




\subsubsection{Primary data from NanoString nCounter experiments - siRNA injection}

(Figure 3.12)

Primary NanoString data analyzing the influence of abolished miR processing by siRNA injection on the stability of germ cell specific mRNAs for two independent experiments is shown in Table 6.6.

Table 6.6 Primary data of RNAs analyzed in NanoString experiments - siRNA injection

\begin{tabular}{|c|c|c|c|c|}
\hline & \multicolumn{2}{|c|}{ experiment 1 , stage 11} & \multicolumn{2}{|c|}{ experiment 2, stage 11} \\
\hline RNA name & uninjected & siRNA (100fM) & uninjected & siRNA (100fM) \\
\hline ArfGAP & 3850 & 1790 & 1025 & 1040 \\
\hline Bic-c & 652 & 480 & 441 & 485 \\
\hline CPEB1a & 1241 & 12234 & 1546 & 12040 \\
\hline Cdk5r2 & 440 & 419 & 817 & 1190 \\
\hline Chk1 & 1751 & 1037 & 1499 & 2042 \\
\hline Cyclin A1 & 6638 & 10676 & 7362 & 18068 \\
\hline Cyclin B2 & 19928 & 26949 & 10329 & 23481 \\
\hline DAZAP2 & 4517 & 3130 & 1759 & 2014 \\
\hline Dazl CDS & 1710 & 1931 & 1567 & 2603 \\
\hline Dazl_UTR & 821 & 888 & 858 & 1171 \\
\hline Dead South & 5900 & 4112 & 2658 & 3441 \\
\hline Eg6/Spire2 & 984 & 451 & 1525 & 1096 \\
\hline Fibronectin 1 & 48946 & 22181 & 5438 & 3787 \\
\hline Fluc-ORF & 9 & 1 & 2 & 3 \\
\hline Fyn & 849 & 599 & 222 & 327 \\
\hline G6PDH & 3399 & 1853 & 1805 & 2116 \\
\hline GFP-ORF & 8 & 10 & 14 & 9 \\
\hline GK & 801 & 508 & 360 & 526 \\
\hline GS17 & 17130 & 17369 & 11076 & 18349 \\
\hline GapDH & 1664 & 959 & 754 & 1064 \\
\hline Grip2 & 4218 & 3222 & 2226 & 2752 \\
\hline $\mathrm{H} 4$ & 55 & 51 & 41 & 65 \\
\hline HPRT & 2009 & 1836 & 861 & 3031 \\
\hline Kif13b & 2684 & 1629 & 792 & 785 \\
\hline Lefty A & 1303 & 830 & 1120 & 878 \\
\hline Lefty B & 907 & 671 & 758 & 408 \\
\hline NWASP & 2672 & 1679 & 703 & 674 \\
\hline Nek2b & 1149 & 1078 & 619 & 1371 \\
\hline ODC & 138761 & 69046 & 31425 & 35325 \\
\hline PRDM1 & 2738 & 1570 & 1103 & 1259 \\
\hline PTB & 11825 & 8177 & 6372 & 8470 \\
\hline Pgam1 & 2613 & 3954 & 2140 & 4609 \\
\hline Rluc-ORF & 3 & 5 & 2 & 6 \\
\hline Rtn3 & 3491 & 1654 & 750 & 719 \\
\hline Syntabulin & 144 & 100 & 190 & 199 \\
\hline Trim36/Haprin & 226 & 157 & 105 & 106 \\
\hline VegT & 48499 & 32809 & 24876 & 20902 \\
\hline Velo1 & 2658 & 3033 & 2291 & 3556 \\
\hline Velo1 isoform & 1047 & 1046 & 777 & 1098 \\
\hline $\mathrm{Vg} 1$ & 2692 & 1313 & 3365 & 4432 \\
\hline Wnt8 & 8231 & 6182 & 4159 & 3987 \\
\hline XNIF & 11397 & 6108 & 3004 & 2380 \\
\hline XVLG1 & 8821 & 4272 & 1880 & 2373 \\
\hline Xcat2 & 177 & 116 & 320 & 242 \\
\hline Xdead end_CDS & 2199 & 5132 & 2093 & 11699 \\
\hline Xdead end_UTR & 153 & 489 & 109 & 457 \\
\hline $\mathrm{Xnr5a/b}$ & 186 & 173 & 76 & 476 \\
\hline Xnr6b & 56 & 59 & 77 & 131 \\
\hline Xpat & 9542 & 3962 & 1844 & 2102 \\
\hline Xwnt11 & 14535 & 8002 & 4248 & 2527 \\
\hline b-actin & 195108 & 103824 & 37311 & 43659 \\
\hline fatvg & 3792 & 2120 & 1430 & 2055 \\
\hline fibronectin receptor & 84 & 65 & 9 & 2 \\
\hline germes & 687 & 319 & 600 & 956 \\
\hline lacZ-fragment & 30 & 29 & 30 & 63 \\
\hline laminB1 & 30589 & 16617 & 5885 & 6842 \\
\hline plk3 & 11840 & 5467 & 4918 & 4046 \\
\hline $\mathrm{xC}$-cadherin & 23753 & 11832 & 6390 & 5567 \\
\hline$x E$-cadherin & 17435 & 8848 & 3070 & 2853 \\
\hline NEG_A & 4 & 6 & 4 & 8 \\
\hline NEG_B & 5 & 7 & 2 & 12 \\
\hline NEG C & 15 & 8 & 11 & 6 \\
\hline NEG_D & 25 & 13 & 9 & 11 \\
\hline NEG E & 10 & 4 & 6 & 5 \\
\hline NEG F & 13 & 13 & 6 & 22 \\
\hline NEG_G & 7 & 6 & 7 & 8 \\
\hline NEG_H & 15 & 5 & 11 & 15 \\
\hline POS_A & 11624 & 12328 & 26664 & 24115 \\
\hline POS B & 7220 & 7467 & 10516 & 9293 \\
\hline POS C & 2309 & 2197 & 3051 & 2848 \\
\hline POS_D & 369 & 420 & 557 & 505 \\
\hline POS_E & 94 & 82 & 162 & 145 \\
\hline POSF & 34 & 32 & 51 & 46 \\
\hline
\end{tabular}




\subsubsection{Processed data from NanoString nCounter analysis - siRNA injection}

(Figure 3.12)

Fold changes over uninjected embryos for experiment 1 and experiment 2 were calculated and are shown in Table 6.7. Averaged fold changes over uninjected embryos and standard deviation are shown in Table 6.8.

Table 6.7 Fold changes over uninjected embryos of two independent experiments

\begin{tabular}{|c|c|c|c|c|}
\hline & \multicolumn{2}{|c|}{ experiment 1 , stage 11} & \multicolumn{2}{|c|}{ experiment 2 , stage 11} \\
\hline RNA name & uninjected & siRNA (100fM) & uninjected & siRNA (100fM) \\
\hline ArfGAP & 1.0 & 0.9 & 1.0 & 0.9 \\
\hline Bic-c & 1.0 & 1.5 & 1.0 & 0.9 \\
\hline CPEB1a & 1.0 & 19.9 & 1.0 & 6.7 \\
\hline Cdk5r2 & 1.0 & 2.0 & 1.0 & 1.2 \\
\hline Chk1 & 1.0 & 1.2 & 1.0 & 1.2 \\
\hline Cyclin A1 & 1.0 & 3.1 & 1.0 & 2.1 \\
\hline Cyclin B2 & 1.0 & 2.6 & 1.0 & 1.9 \\
\hline DAZAP2 & 1.0 & 1.3 & 1.0 & 1.0 \\
\hline Dazl_CDS & 1.0 & 2.2 & 1.0 & 1.4 \\
\hline Dazl_UTR & 1.0 & 2.2 & 1.0 & 1.2 \\
\hline Dead South & 1.0 & 1.3 & 1.0 & 1.1 \\
\hline Eg6/Spire2 & 1.0 & 0.9 & 1.0 & 0.6 \\
\hline Fibronectin 1 & 1.0 & 0.9 & 1.0 & 0.6 \\
\hline Fluc-ORF & 1.0 & 0.6 & 1.0 & 0.0 \\
\hline Fyn & 1.0 & 1.4 & 1.0 & 1.3 \\
\hline G6PDH & 1.0 & 1.1 & 1.0 & 1.0 \\
\hline GFP-ORF & 1.0 & 1.0 & 1.0 & 1.0 \\
\hline GK & 1.0 & 1.3 & 1.0 & 1.2 \\
\hline GS17 & 1.0 & 2.0 & 1.0 & 1.4 \\
\hline GapDH & 1.0 & 1.1 & 1.0 & 1.2 \\
\hline Grip2 & 1.0 & 1.5 & 1.0 & 1.1 \\
\hline $\mathrm{H} 4$ & 1.0 & 1.0 & 1.0 & 1.4 \\
\hline HPRT & 1.0 & 1.8 & 1.0 & 3.0 \\
\hline Kif13b & 1.0 & 1.2 & 1.0 & 0.8 \\
\hline Lefty A & 1.0 & 1.3 & 1.0 & 0.7 \\
\hline Lefty B & 1.0 & 1.5 & 1.0 & 0.4 \\
\hline NWASP & 1.0 & 1.2 & 1.0 & 0.8 \\
\hline Nek2b & 1.0 & 1.9 & 1.0 & 1.9 \\
\hline ODC & 1.0 & 1.0 & 1.0 & 1.0 \\
\hline PRDM1 & 1.0 & 1.1 & 1.0 & 1.0 \\
\hline PTB & 1.0 & 1.3 & 1.0 & 1.1 \\
\hline Pgam1 & 1.0 & 3.0 & 1.0 & 1.8 \\
\hline Rluc-ORF & 1.0 & 1.0 & 1.0 & 1.0 \\
\hline Rtn3 & 1.0 & 0.9 & 1.0 & 0.8 \\
\hline Syntabulin & 1.0 & 1.9 & 1.0 & 0.9 \\
\hline Trim36/Haprin & 1.0 & 1.6 & 1.0 & 0.8 \\
\hline VegT & 1.0 & 1.3 & 1.0 & 0.7 \\
\hline Velo1 & 1.0 & 2.2 & 1.0 & 1.3 \\
\hline Velo1 isoform & 1.0 & 2.0 & 1.0 & 1.2 \\
\hline Vg1 & 1.0 & 0.9 & 1.0 & 1.1 \\
\hline Wnt8 & 1.0 & 1.4 & 1.0 & 0.8 \\
\hline XNIF & 1.0 & 1.0 & 1.0 & 0.7 \\
\hline XVLG1 & 1.0 & 0.9 & 1.0 & 1.1 \\
\hline Xcat2 & 1.0 & 1.6 & 1.0 & 0.6 \\
\hline Xdead end_CDS & 1.0 & 4.6 & 1.0 & 4.8 \\
\hline Xdead end_UTR & 1.0 & 10.0 & 1.0 & 3.9 \\
\hline Xnr5a/b & 1.0 & 2.4 & 1.0 & 6.3 \\
\hline Xnr6b & 1.0 & 1.0 & 1.0 & 1.5 \\
\hline Xpat & 1.0 & 0.8 & 1.0 & 1.0 \\
\hline Xwnt11 & 1.0 & 1.1 & 1.0 & 0.5 \\
\hline b-actin & 1.0 & 1.0 & 1.0 & 1.0 \\
\hline fatvg & 1.0 & 1.1 & 1.0 & 1.2 \\
\hline fibronectin receptor & 1.0 & 4.2 & 1.0 & 2.9 \\
\hline germes & 1.0 & 0.9 & 1.0 & 1.4 \\
\hline lacZ-fragment & 1.0 & 1.0 & 1.0 & 1.0 \\
\hline laminB1 & 1.0 & 1.0 & 1.0 & 1.0 \\
\hline plk3 & 1.0 & 0.9 & 1.0 & 0.7 \\
\hline $\mathrm{xC}$-cadherin & 1.0 & 1.0 & 1.0 & 0.7 \\
\hline $\mathrm{xE}$-cadherin & 1.0 & 1.0 & 1.0 & 0.8 \\
\hline
\end{tabular}


Table 6.8 Averaged fold changes over uninjected embryos of two independent experiments and calculated standard deviation

\begin{tabular}{|c|c|c|}
\hline & fold char & exp.1+2 \\
\hline Gene name & uninjected & siRNA (200fmol) \\
\hline ArfGAP & 1.0 & 0.9 \\
\hline Bic-c & 1.0 & 1.2 \\
\hline CPEB1a & 1.0 & 13.3 \\
\hline Cdk5r2 & 1.0 & 1.6 \\
\hline Chk1 & 1.0 & 1.2 \\
\hline Cyclin A1 & 1.0 & 2.6 \\
\hline Cyclin B2 & 1.0 & 2.3 \\
\hline DAZAP2 & 1.0 & 1.2 \\
\hline Dazl_CDS & 1.0 & 1.8 \\
\hline Dazl_UTR & 1.0 & 1.7 \\
\hline Dead South & 1.0 & 1.2 \\
\hline Eg6/Spire2 & 1.0 & 0.8 \\
\hline Fibronectin 1 & 1.0 & 0.7 \\
\hline Fluc-ORF & 1.0 & 0.3 \\
\hline Fyn & 1.0 & 1.3 \\
\hline G6PDH & 1.0 & 1.0 \\
\hline GFP-ORF & 1.0 & 1.0 \\
\hline GK & 1.0 & 1.3 \\
\hline GS17 & 1.0 & 1.7 \\
\hline GapDH & 1.0 & 1.2 \\
\hline Grip2 & 1.0 & 1.3 \\
\hline $\mathrm{H} 4$ & 1.0 & 1.0 \\
\hline HPRT & 1.0 & 2.4 \\
\hline Kif13b & 1.0 & 1.0 \\
\hline Lefty A & 1.0 & 1.0 \\
\hline Lefty B & 1.0 & 1.0 \\
\hline NWASP & 1.0 & 1.0 \\
\hline Nek2b & 1.0 & 1.9 \\
\hline ODC & 1.0 & 1.0 \\
\hline PRDM1 & 1.0 & 1.0 \\
\hline PTB & 1.0 & 1.2 \\
\hline Pgam1 & 1.0 & 2.4 \\
\hline Rluc-ORF & 1.0 & 1.0 \\
\hline Rtn3 & 1.0 & 0.9 \\
\hline Syntabulin & 1.0 & 1.4 \\
\hline Trim36/Haprin & 1.0 & 1.2 \\
\hline VegT & 1.0 & 1.0 \\
\hline Velo1 & 1.0 & 1.8 \\
\hline Velo1 isoform & 1.0 & 1.6 \\
\hline Vg1 & 1.0 & 1.0 \\
\hline Wnt8 & 1.0 & 1.1 \\
\hline XNIF & 1.0 & 0.9 \\
\hline XVLG1 & 1.0 & 1.0 \\
\hline Xcat2 & 1.0 & 1.1 \\
\hline Xdead end_CDS & 1.0 & 4.7 \\
\hline Xdead end UTR & 1.0 & 7.0 \\
\hline $\mathrm{Xnr5a/b}$ & 1.0 & 4.4 \\
\hline Xnr6b & 1.0 & 1.0 \\
\hline Xpat & 1.0 & 0.9 \\
\hline Xwnt11 & 1.0 & 0.8 \\
\hline b-actin & 1.0 & 1.0 \\
\hline fatvg & 1.0 & 1.2 \\
\hline fibronectin receptor & 1.0 & 3.5 \\
\hline germes & 1.0 & 1.1 \\
\hline lacZ-fragment & 1.0 & 1.0 \\
\hline laminB1 & 1.0 & 1.0 \\
\hline plk3 & 1.0 & 0.8 \\
\hline xC-cadherin & 1.0 & 0.8 \\
\hline xE-cadherin & 1.0 & 0.9 \\
\hline
\end{tabular}

\begin{tabular}{|c|c|c|}
\hline & \multicolumn{2}{|c|}{ std dev exp.1 + 2} \\
\hline Gene name & uninjected & $\begin{array}{c}\text { siRNA } \\
(200 \mathrm{fmol})\end{array}$ \\
\hline ArfGAP & 0.0 & 0.0 \\
\hline Bic-c & 0.0 & 0.4 \\
\hline CPEB1a & 0.0 & 9.3 \\
\hline Cdk5r2 & 0.0 & 0.6 \\
\hline Chk1 & 0.0 & 0.0 \\
\hline Cyclin A1 & 0.0 & 0.7 \\
\hline Cyclin B2 & 0.0 & 0.5 \\
\hline DAZAP2 & 0.0 & 0.3 \\
\hline Dazl_CDS & 0.0 & 0.6 \\
\hline Dazl_UTR & 0.0 & 0.7 \\
\hline Dead South & 0.0 & 0.2 \\
\hline Eg6/Spire2 & 0.0 & 0.2 \\
\hline Fibronectin 1 & 0.0 & 0.2 \\
\hline Fluc-ORF & 0.0 & 0.4 \\
\hline Fyn & 0.0 & 0.1 \\
\hline G6PDH & 0.0 & 0.0 \\
\hline GFP-ORF & 0.0 & 0.0 \\
\hline GK & 0.0 & 0.0 \\
\hline GS17 & 0.0 & 0.4 \\
\hline GapDH & 0.0 & 0.1 \\
\hline Grip2 & 0.0 & 0.3 \\
\hline $\mathrm{H} 4$ & 0.0 & 0.0 \\
\hline HPRT & 0.0 & 0.9 \\
\hline Kif13b & 0.0 & 0.2 \\
\hline Lefty A & 0.0 & 0.4 \\
\hline Lefty B & 0.0 & 0.7 \\
\hline NWASP & 0.0 & 0.3 \\
\hline Nek2b & 0.0 & 0.0 \\
\hline ODC & 0.0 & 0.0 \\
\hline PRDM1 & 0.0 & 0.1 \\
\hline PTB & 0.0 & 0.1 \\
\hline Pgam1 & 0.0 & 0.8 \\
\hline Rluc-ORF & 0.0 & 0.0 \\
\hline Rtn3 & 0.0 & 0.1 \\
\hline Syntabulin & 0.0 & 0.7 \\
\hline Trim36/Haprin & 0.0 & 0.6 \\
\hline VegT & 0.0 & 0.4 \\
\hline Velo1 & 0.0 & 0.6 \\
\hline Velo1 isoform & 0.0 & 0.6 \\
\hline Vg1 & 0.0 & 0.1 \\
\hline Wnt8 & 0.0 & 0.4 \\
\hline XNIF & 0.0 & 0.3 \\
\hline XVLG1 & 0.0 & 0.1 \\
\hline Xcat2 & 0.0 & 0.7 \\
\hline Xdead end_CDS & 0.0 & 0.1 \\
\hline Xdead end_UTR & 0.0 & 4.3 \\
\hline Xnr5a/b & 0.0 & 2.8 \\
\hline Xnr6b & 0.0 & 0.0 \\
\hline Xpat & 0.0 & 0.1 \\
\hline Xwnt11 & 0.0 & 0.4 \\
\hline b-actin & 0.0 & 0.0 \\
\hline fatvg & 0.0 & 0.1 \\
\hline fibronectin receptor & 0.0 & 0.9 \\
\hline germes & 0.0 & 0.3 \\
\hline lacZ-fragment & 0.0 & 0.0 \\
\hline laminB1 & 0.0 & 0.0 \\
\hline plk3 & 0.0 & 0.1 \\
\hline $\mathrm{xC}$-cadherin & 0.0 & 0.2 \\
\hline$x E$-cadherin & 0.0 & 0.1 \\
\hline
\end{tabular}




\subsection{XDE-LE mutant used for the analysis of XDazl, XDE and ElrB1 binding}

(Figure 3.9)

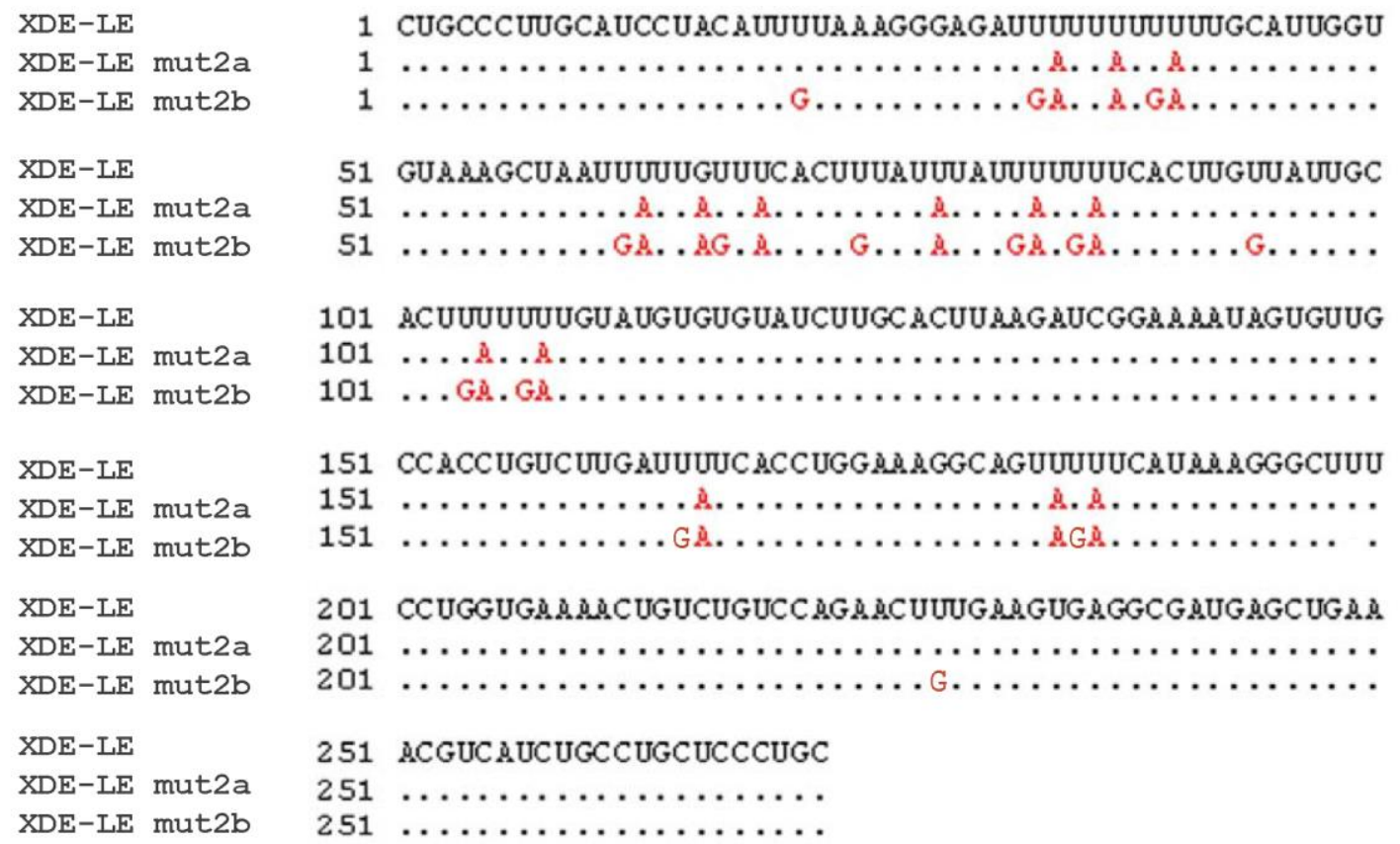

Figure 6.7. Mutagenesis of putative XDazl, XDE and EIrA/B binding sites in the XDE-LE.

Sequence alignment of the wild type $X D E-L E$ and the mutants mut2a and mut2b, harboring nucleotide exchanges in U-rich elements interrupted with $A$ residues and $G$ residues. The mutants were generated by $P$. Arthur. (Arthur 2008) 


\section{Curriculum Vitae}

Name:

Juliane Pfennig, born Wellner

Date of Birth:

11.07.1983

Place of Birth:

Erfurt, Germany

Email:

Juliane.Wellner@gmx.de

\section{Education:}

$10.2010-09.2014$

Doctoral studies: "XDazl function in RNA metabolism in Xenopus laevis"

Dept. of Developmental Biochemistry, Georg August University of Göttingen, Germany. Supervisor: Prof. Dr. T. Pieler.

04.03.2011

Diploma, Friedrich Schiller University, Jena, Germany

2010

Diploma studies: "Characterization of a conditional Wt1 knock out mouse following germline deletion".

Dept. of Molecular Genetics, Friedrich Schiller University, Jena, Germany. Supervisor: Prof. Dr. C. Englert.

$2004-2010$

Studies in Biology, Friedrich Schiller University, Jena, Germany

$2002-2004$

Studies in Pharma-Biotechnology, Fachhochschule Jena

06.2002

Abitur, Oskar-Gründler-Gymnasium Gebesee, Germany

\section{Publications:}

Hartmann N, Reichwald K, Lechel A, Graf M, Kirschner J, Dorn A, Terzibasi E, Wellner J, Platzer M, Rudolph KL, Cellerino A, Englert C.Telomeres shorten while Tert expression increases during ageing of the short-lived fish Nothobranchius furzeri. Mech Ageing Dev. 2009 May;130(5):290-6 